\title{
The graphity of cognitive problems in epilepsy
}

Citation for published version (APA):

Vaessen, M. J. (2012). The graphity of cognitive problems in epilepsy. [Doctoral Thesis, Maastricht University]. BOXPress. https://doi.org/10.26481/dis.20120329mv

Document status and date:

Published: 01/01/2012

DOI:

10.26481/dis.20120329mv

Document Version:

Publisher's PDF, also known as Version of record

\section{Please check the document version of this publication:}

- A submitted manuscript is the version of the article upon submission and before peer-review. There can be important differences between the submitted version and the official published version of record.

People interested in the research are advised to contact the author for the final version of the publication, or visit the DOI to the publisher's website.

- The final author version and the galley proof are versions of the publication after peer review.

- The final published version features the final layout of the paper including the volume, issue and page numbers.

Link to publication

\footnotetext{
General rights rights.

- You may freely distribute the URL identifying the publication in the public portal. please follow below link for the End User Agreement:

www.umlib.nl/taverne-license

Take down policy

If you believe that this document breaches copyright please contact us at:

repository@maastrichtuniversity.nl

providing details and we will investigate your claim.
}

Copyright and moral rights for the publications made accessible in the public portal are retained by the authors and/or other copyright owners and it is a condition of accessing publications that users recognise and abide by the legal requirements associated with these

- Users may download and print one copy of any publication from the public portal for the purpose of private study or research.

- You may not further distribute the material or use it for any profit-making activity or commercial gain

If the publication is distributed under the terms of Article $25 \mathrm{fa}$ of the Dutch Copyright Act, indicated by the "Taverne" license above, 
The graphity of cognitive problems

in epilepsy 
(C) Maarten Vaessen, Maastricht 2012

Layout: Ilona Punt, Maarten Vaessen

Cover design: Remko Rinia, linecreation.nl

Production: BOXPress BV || Proefschriftmaken.nl

ISBN: 978-90-8891-394-5

The printing of this thesis was financially supported by the School for Mental Health and Neuroscience, Nationaal Epilepsie Fonds, Eisai B.V. and UCB Pharma B.V. 


\title{
The graphity of cognitive problems in epilepsy
}

\author{
PROEFSCHRIFT
}

ter verkrijging van de graad van doctor aan de Universiteit Maastricht, op gezag van de Rector Magnificus, Prof. Mr. G.P.M.F. Mols volgens het besluit van het College van Decanen, in het openbaar te verdedigen op donderdag 29 maart 2012 om 12:00 uur

door

Maarten Johannes Vaessen

geboren te Hoensbroek op 4 juli 1981 


\section{Promotor}

Prof. Dr. A.P. Aldenkamp

\section{Co-promotores}

Dr. Ir. W.H. Backes

Dr. P.A.M. Hofman

\section{Beoordelingscommissie}

Prof. Dr. J. Wildberger (voorzitter)

Prof. Dr. E. Achten (Universiteit Gent, Belgie)

Prof. Dr. R. Goebel

Prof. Dr. G. van Merode

Prof. Dr. R. van Oostenbrugge 


\section{CONTENTS}

$\begin{array}{lll}\text { Chapter } 1 & \text { General introduction } & 7\end{array}$

Chapter 2 The effect and reproducibility of different clinical DTI gradient 29 sets on small world brain connectivity measures Neurolmage. 2010, 51(3):1106-16

Chapter 3 Loss of functional network efficiency associated with cognitive decline in chronic epilepsy Neurology. 2011, 77(10):938-44

Chapter 4 White matter network abnormalities are associated with cognitive decline in chronic epilepsy

Cerebral Cortex 2011; doi: 10.1093/cercor/bhr298, Epub ahead of print

Chapter 5 Abnormal modular organization of functional networks in cognitively impaired children with frontal lobe epilepsy

Submitted

Chapter 6 Connectional abnormalities of functional and structural networks in childhood frontal lobe epilepsy

In preparation

Chapter 7 General discussion

Summary

Samenvatting

Dankwoord

List of publications

Curriculum Vitae 

CHAPTER 1

General introduction 


\section{EPILEPSY}

Epilepsy is one of the most prevalent neurological disorders worldwide. It affects 1-2\% percent of the population at some point in their lives. In the Netherlands alone, approximately 110,000 persons have some form of epilepsy at any given moment (NEF, 2009).

An epileptic seizure is defined as the paroxysmal manifestation of symptoms due to abnormal excessive or synchronous neuronal discharges in the brain (Fisher et al., 2005; Berg et al., 2010). Clinically, a person is given a diagnosis of epilepsy after at least two unprovoked epileptic seizures (Blume et al., 2001), although there are several circumstances after which the diagnosis is made after one seizure (for example when EEG confirms absence seizures in children) and in some epilepsies seizures are rare or even absent such as in the Landau Kleffner syndrome. There are many possible different causes and clinical expressions of epilepsy and it is therefore not a single disease entity, but rather a description of a large number of diseases with common symptoms (Panayiotopoulos, 2002).

Epileptic seizures are typically classified by both the underlying cause and the location and nature of the seizure origin according to the classification of the International League Against Epilepsy (ILAE) (ILAE, 1989).

The underlying etiology can be classified as either genetic or structural/metabolic, but is in many cases of unknown cause (Berg et al., 2010). Genetic epilepsies are best understood as the direct result of a known or presumed genetic defect in which seizures are the core symptom of the disorder. Epilepsies with a structural of metabolic cause are characterized by the presence of a distinct structural lesion, metabolic condition or disease that has been demonstrated to be associated with a substantially increased risk of developing epilepsy. This does not preclude that there is no genetic contribution, however there is a separate disorder interposed between the genetic defect and the epilepsy, such as the occurrence of a stroke or a tumor. The epilepsies of unknown cause (which are also known as non-symtomatic or cryptogenic epilepsy), designates the epilepsies for which the nature of the underlying cause is as yet unknown. The epilepsy may have a fundamental genetic defect at its core or it may be the consequence of a separate as yet unrecognized disorder.

Seizures can be classified by the mode of seizure onset. Here a distinction can be made between generalized seizures and focal seizures. Generalized epileptic seizures originate at some point within, and rapidly engaging bilaterally distributed networks. Such bilateral networks can include cortical and subcortical structures, but do not necessarily include the entire cortex. Critically, the localization and lateralization are not consistent between one seizure and another. Focal epileptic seizures, on the other hand, originate within networks limited to one hemisphere. They may be discretely localized or more widely distributed. Focal seizures may originate in subcortical structures. For each seizure type, ictal onset is consistent from one seizure to another. 
The unpredictability and sudden occurrence of a seizure frequently creates a great social burden to the patient and those who surround him. Consciousness is often lost during a seizure and can therefore cause physical harm to the patient. Furthermore, living with the uncertainty of not knowing when or whether the next seizure will occur can cause psychological stress. In addition, epilepsy patients may be hindered in their daily live by driving and working restrictions, the side-effects of the medication and psychological and cognitive complications. Although all of the above mentioned problems can be serious, many patients rank their cognitive impairments highest on their list of complaints (Chaplin et al., 1992; Baker et al., 1997; Fisher et al., 2000; Vlooswijk et al., 2010).

\section{EPILEPSY AND COGNITION}

Cognitive problems, or cognitive co-morbidity, are reported on virtually all cognitive domains in epilepsy. Classically, temporal lobe epilepsy (TLE) has been associated with memory deficits, ranging from impairments in verbal long-term consolidation and retrieval, verbal learning, short term working memory and spatial memory (Vlooswijk et al., 2010). In frontal lobe epilepsy (FLE), cognitive deficits and behavioral disturbances range from impaired attention to difficulties with the more complex behaviors involved in planning, selecting goals, anticipating outcomes, and initiating actions (Braakman et al., 2011). However, all sorts of epilepsies may be associated with so called encephalopathy: "the epileptic activity itself may contribute to severe cognitive and behavioral impairments above and beyond what might be expected from the underlying pathology alone,..., and these can worsen over time" (Berg et al., 2010).

Currently, little is known about the mechanisms that cause cognitive deficits in patients with epilepsy. Naturally, much research has focused on clinical factors that might contribute to the development of cognitive disorders. For instance, the role of seizure frequency and seizure severity (Dodrill, 2002), age at onset of the seizures (Hermann et al., 2002), drug use (Vermeulen and Aldenkamp, 1995) and status epilepticus (persistent interictal epileptic brain activity) (Aldenkamp and Arends, 2004). However, results have been mixed and as yet no solid relationship between cognitive decline and clinical factors has been demonstrated. This issue is further complicated by studies indicating that cognitive deficits can already be present before occurrence of the first epileptic seizure (Hermann et al., 2006; Taylor et al., 2010). Therefore, the question of causality between seizures and cognitive deficits is raised. Do seizures cause cognitive problems or are seizures and cognitive problems both symptoms of the same underlying pathology? To address this question recent research (including this thesis) has focused on the neuronal mechanism underlying cognitive deficits. 


\section{BRAIN AND COGNITION}

The term cognition ("cognoscere" in Latin, which means, "to know", "to conceptualize" or "to recognize") refers to the mental functions, mental processes (thoughts) and states of an intelligent being. But how are cognitive functions produced by the brain? Historically, most investigations have focused on the location of brain regions that support a cognitive function. The earliest traces of serious investigation from the "localizationists" view go back to John Hughlings Jackson (18351911) who investigated epilepsy patients with brain damage and proposed that specific functions were localized to specific areas of the brain, or to Paul Broca (18241881) and Carl Wernicke (1848-1905) who's research on patients with specific language problems led to the detection of the language areas of the brain.

Much of the early research in this field was only possible by studying the post-mortem brain of patients with specific lesions, or by performing lesion studies in animal models (Geschwind, 1965b, a; Luria, 1976; Damasio and Damasio, 1989). Only with the invention of the EEG in the early $20^{\text {th }}$ century (Haas, 2003), did it become possible to link large-scale electrical recordings of the brain to behavior and cognition (Niedermeyer, 1997). Much can be said about EEG research and the brain, especially in the field of epilepsy where EEG is now the standard clinical tool for seizure diagnosis and classification. However, it is beyond the scope of this chapter to provide an elaborate discussion on EEG, epilepsy and cognition. Instead, the focus of this thesis is on another non-invasive neuro-imaging technique, Magnetic Resonance Imaging (MRI). In the next sections of this chapter an introduction will be given to this technique, how it can be used to probe brain functionality and axonal organization and how it might be used to investigate patients with epilepsy and cognitive problems.

\section{MRI}

Magnetic Resonance Imaging is a noninvasive method to obtain a detailed view of structures, such as the brain, in the living body. Due to its many contrast mechanisms (e.g. T1, T2, proton density) reflecting the magneto kinetic properties of various brain structures, MRI is able to highlight different structural and functional aspects of the brain.

Historically, MRI has mainly been used to study the complex anatomical structure of the brain. In epilepsy, high resolution $\mathrm{T} 1$ and $\mathrm{T} 2$ images have enabled the detection of for example cerebro-vascular lesions, brain tumors, developmental disorders and traumatic lesions (Salmenpera and Duncan, 2005; Urbach, 2005), see Figure 1.1. This has had a significant impact on clinical research and practice, by establishing better diagnosis, treatment decisions and monitoring of treatment effects (Kuzniecky and Jackson, 2005). 
Although macro-structural imaging can provide images of the brain with high detail, the image is inherently static: a snapshot is made, which does not provide information on the ever-ongoing dynamics in the brain. Functional Magnetic Resonance Imaging is an MR technique that can provide information on the activity of brain regions and networks.

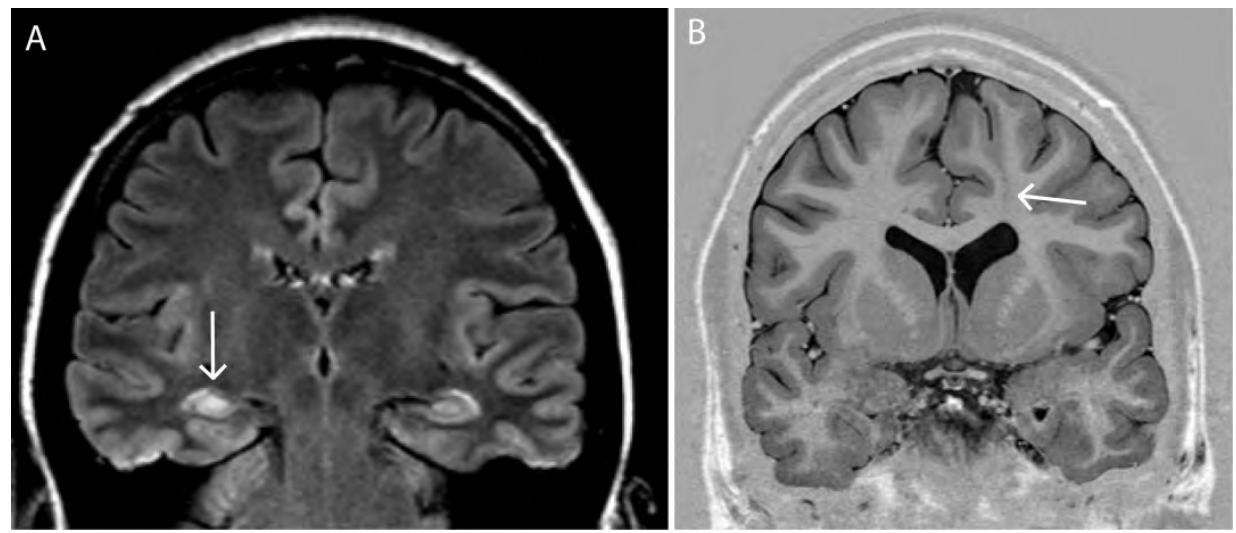

Figure 1.1 MR visible lesions associated with epilepsy. (A) Coronal fluid-attenuated inversion recovery (FLAIR) image; arrow marks the hippocampal sclerosis. (B) Coronal inversion recovery T1weighted image; arrow marks the focal cortical dys plasia.

\section{FUNCTIONAL IMAGING: BOLD SIGNAL}

Functional MR techniques can potentially be implemented using many different contrast mechanisms (Belliveau et al., 1991; Kwong et al., 1992; Brown et al., 2007), although for neuroscience applications this is usually done with Blood Oxygenation Level Dependent (BOLD) signal imaging (Ogawa et al., 1990; Turner et al., 1991; Kwong et al., 1992). This technique utilizes the physiological mechanism where neural activity triggers an increase in supply of fresh (oxygenated) blood to the active region, and is thus an indirect measurement of neuronal activity. The change in ratio between oxygenated and (paramagnetic) de-oxygenated blood can be measured with MRI (T2* weighted imaging). By utilizing MR techniques that can image the entire brain volume in a matter of seconds (Stehling et al., 1991; Tsao, 2010), a pattern of activation over time of the entire brain can be obtained.

The first (and still foremost) application of functional imaging was that of localization of functionally specialized regions in the brain (Kwong et al., 1992). In a typical fMRI experiment a subject, while lying in the scanner, is asked to perform a certain task which is contrasted with periods of rest. The difference in signal intensity between the task and rest conditions can then be calculated and regions where the signal intensity 
is significantly higher during the task than during rest can be said to be activated. This type of experiment has provided a vast amount of information on the functional topography of the human brain (Fox and Lancaster, 2002): for most tasks it is now known what brain regions they activate.

This kind of functional localization in the brain rests upon the statistical analysis of a model signal of brain responses (the regressor) and the measured BOLD signal. Based upon the experimental paradigm (for instance a task-rest block design), a model signal is constructed and regressed against the BOLD signal time course of all brain voxels. The voxels where the association between the model signal and measured signal is highly significant are then said to be activated by the experimental task. See Figure 1.2A. However, with this type of analysis each location in the brain is tested separately for activation. As such, conventional $f M R I$ is not able to provide information on communication between brain regions. This could be highly relevant for the study of abnormal brain organization in neurological disorders such as epilepsy, as will be discussed in the next section.

\section{FUNCTIONAL CONNECTIVITY MRI}

Several researchers have noted that in addition to the model signal in conventional $\mathrm{fMRI}$, the intrinsic BOLD signal fluctuation of a brain region can also be used as a regressor (Friston, 1994; Biswal et al., 1995), see Figure 1.2B. By measuring the association (or level of synchronicity) between different brain regions we can obtain insights into the large-scale functional organization of the brain. One of the first experiments in this field showed that, during rest, left and right hemispheric regions of the primary motor cortex had a high functional connectivity: the BOLD time signals showed a high level of correlation even though the subject was not engaged in any sort of motor activity (Biswal et al., 1995). At present day, these findings have been replicated over and over and functional connectivity between a large number of brain regions and systems have been reported (Smith et al., 2009).

Still, the exact origin of functional correlation in the BOLD signal is incompletely understood (Fox and Raichle, 2007). It is generally thought that functional correlations of the BOLD signal are closely related to the inherently ongoing activity of the underlying neuronal populations. This neuronal basis is supported by reports that have directly linked spontaneous BOLD fluctuations to fluctuations in neuronal firing rate (He et al., 2008; Shmuel and Leopold, 2008). Also, functional correlations have been found between brain regions that are also co-activated in several tasks, for example regions known to be involved in motor and visual functions and the default mode network (regions that are de-activated by task fMRI) (Biswal et al., 1995; Damoiseaux et al., 2006; Smith et al., 2009).

An important question in this field is how the pattern of functional connectivity during rest or task is shaped and constrained by the underlying white matter connectivity 
(Honey et al., 2007). This question can be answered directly thanks to another recent development in MRI called Diffusion Weigthed Imaging (DWI).

A

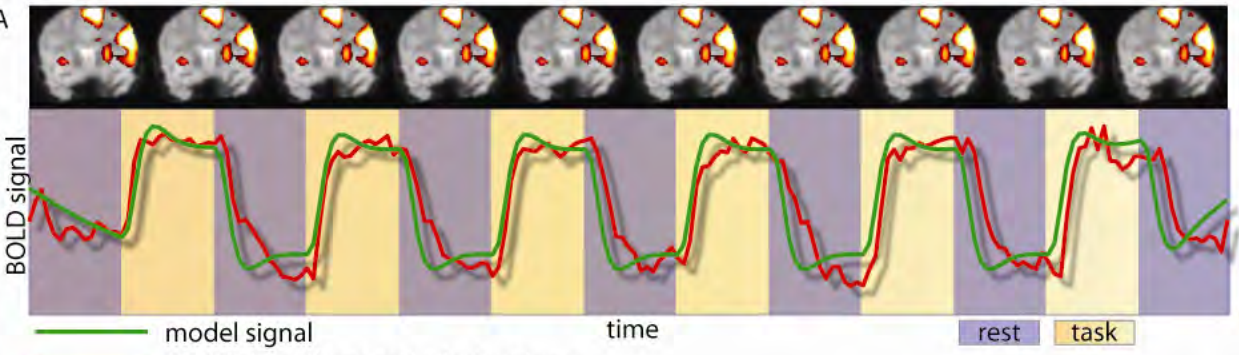

BOLD signal from activated regions

B
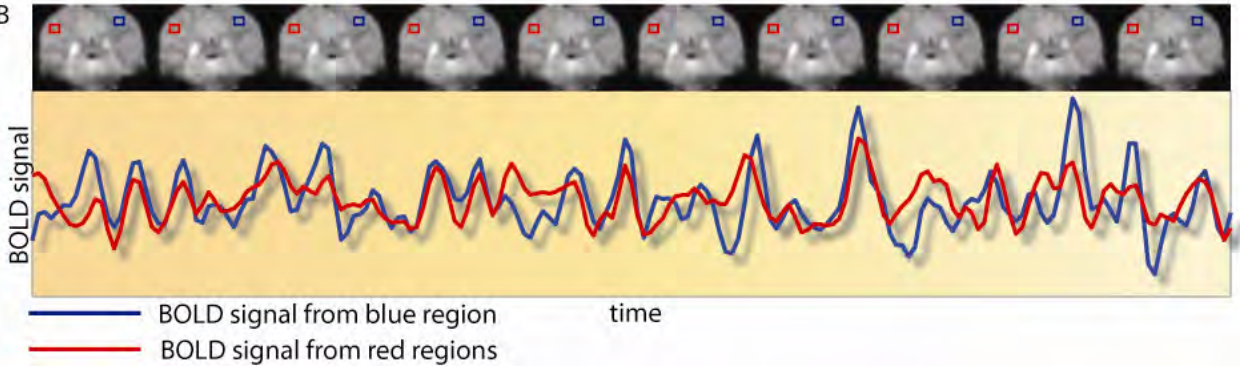

Figure 1.2 (A) Functional activation and the BOLD signal. In this panel, the principle of BOLD activation imaging is depicted. The top row shows actual BOLD (T2*weighted) images over time. The $\mathrm{red} /$ yellow overlay displays where significant activation is found. The lower row displays the design of the task by yellow and purple panels, representing task and rest epochs, respectively. The green line is the regressor based on the task design. Basically, this is the block pattern of the task (yellow/purple panels) convoluted with the Hemodynamic Response Function (HRF). Note how the green line is somewhat delayed compared to the actual onsets of task and rest blocks. This compares nicely to the actual measured BOLD signal (the red line). The BOLD signal is a vascular response which is indeed slow. (B) Functional correlation. Here a similar setup as in panel $A$ is used. However, a resting state fMRI scan is shown, and thus there is no task design. The top row again displays the actual BOLD images over time. The red and blue squares indicate from what region of the brain the BOLD signal is shown in the lower panel (red and blue lines). It is apparent that the two BOLD signals (spontaneous fluctuations) are not equal but are highly synchronous. This indicates that the red and blue regions are functionally connected. 


\section{DIFFUSION WEIGHTED IMAGING}

Information on white matter networks of the brain can be obtained in-vivo, by acquiring diffusion weighted imaging data and subsequently performing fiber tractography. DWI is an MRI technique which enables the measurement of water diffusion in multiple spatial directions. In the brain, the movement of extracellular water molecules is hindered by cellular barriers present in biological tissue such as myelinated axons. Due to directional differences in water diffusion in different structures, DWI is able to provide information on the orientation of white matter. One of the most widely used methods to capture orientation information from DWI data is the Diffusion Tensor model (Le Bihan et al., 2001). For this, a three dimensional ellipsoid form is fitted to the DWI signal. Consequently, the principal axis of the ellipsoid (the direction in which the ellipsoid is most stretched, and most water diffusion has occurred) is assumed to represent the direction of the underlying white matter fiber bundles. Other quantities can also be derives from the diffusion tensor, such as the fractional anisotropy (FA) and mean diffusivity (MD). See Figure 1.3.

FA and MD can provide valuable clinical information on local abnormalities related to various pathologies including Alzheimer's disease (Sundgren et al., 2004) and epilepsy (Yogarajah and Duncan, 2008). MD and FA yield information on the architecture of brain tissue at the voxel level, these parameters do not provide information on the projection of nerve fiber bundles between cerebral areas. Recently, a number of techniques have been developed to investigate the continuity of fiber orientations from voxel to voxel (e.g., streamline tractography) on a whole-brain basis.

The foremost use of fiber tractography is to reconstruct the pathways of the major white matter fiber bundles in the brain. The white matter derives its name from the fact that the myelin surrounding axons is a fatty substance and thus appears white to the eye. The name, however, is somewhat misleading as it suggests a homogeneous substance (matter), while in fact the axonal projections of neurons compose an intricate and complex structural network. Fiber tractography enables us to delineate the white matter into the large fiber bundles of which it is composed. In other words, where white matter displays a more or less uniform intensity on conventional structural MR images, fiber tractography uniquely brings contrast to the white matter. It is, however, not straightforward to derive fiber bundles from diffusion weighted MR images.

The local micro-architecture of the white matter can be derived from models relating measured water diffusion to fiber orientation. As discussed above, the diffusion tensor is one of the most used and most simple models. Recently a number of other models have been proposed, that not only model the principal diffusion direction and thus only one fiber orientation, but model several fiber orientations (Parker et al., 2003; Behrens et al., 2007) or a continuum of fiber orientations (the fiber orientation distribution, FOD, or orientation distribution function ODF) (Alexander, 2005; Tournier et al., 2007). These models depart from the naïve assumption that the white matter in 
Ai

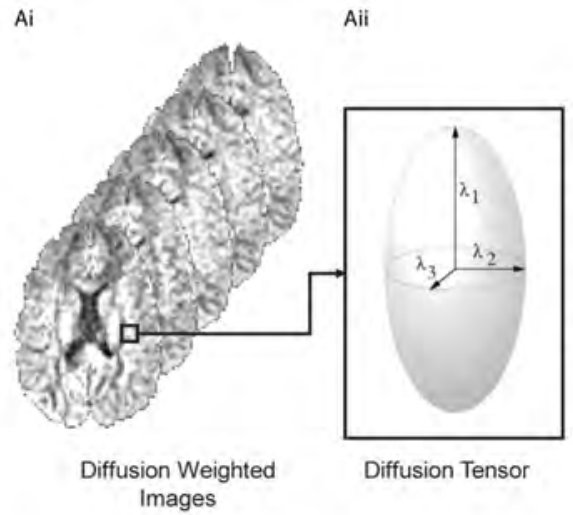

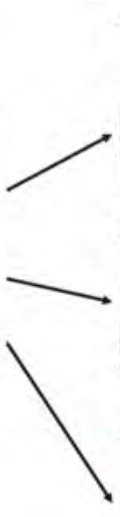

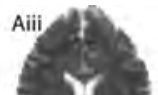

Average

Diffusion

Coefficient
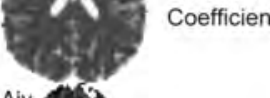

ractional

Anisotropy

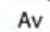

Av

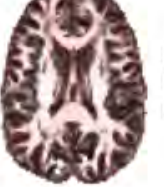

Principal

Diffusion

Direction

Bi
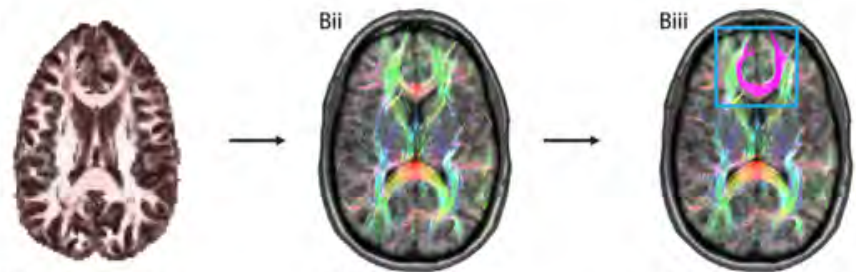

Diffusion direction WM tractography

Fiber bundle selection

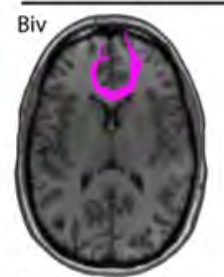

Number of fibers

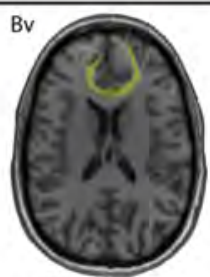

Tract volume

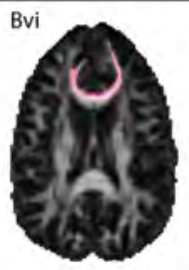

Tract FA

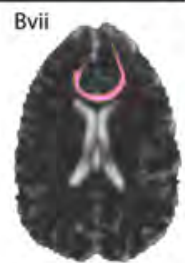

Tract ADC

Figure 1.3 Diffusion weighted imaging and fiber tractography. This composite figure depicts some of the concepts of diffusion weighted imaging and fiber tractography. In panel (Ai), a number of diffusion weighted (echo-planar) images are shown. For each image diffusion is measured in a different direction. The values from a voxel (black square) are fitted to the Diffusion Tensor model (Aii). From this tensor a number of parameters can be derived. The Apparent Diffusion Coefficient (Aiii) (a.k.a the Mean Diffusivity) is the average over the magnitude of the eigenvectors of the tensor, $A D C=\left(\lambda_{1}+\lambda_{2}+\lambda_{3}\right) / 3$. The Fractional Anisotropy (Aiv) quantifies the anisotropy of the tensor (i.e. the discrepancy between the largest eigenvector and the other two), $F A=\sqrt{3 / 2} * \sqrt{\left(\lambda_{1}-\langle\lambda\rangle\right)^{2}+\left(\lambda_{2}-\langle\lambda\rangle\right)^{2}+\left(\lambda_{3}-\langle\lambda\rangle\right)^{2}} / \sqrt{\lambda_{1}^{2}+\lambda_{2}^{2}+\lambda_{3}^{2}}$. The principle diffusion direction (Av) is simply the largest eigenvector of the tensor $\mathrm{v}_{1}$. Panel (B) shows the principle of fiber tractography and fiber quantification. The principal diffusion direction (Bi) (derived from the tensor or more advanced models for fiber directionality) is followed from voxel to voxel creating fiber tracts as shown in the middle (Bii). By applying selection criteria a set of fibers connecting different parts of the brain can be obtained (Biii). A tract can be quantified by various parameters such as the number of fibers (Biv), the volume of the tract (Bv), the mean FA value of the voxel in the tracts (Bvi) or the mean ADC within the tract (Bvii). 
a voxel is oriented along a single direction. In fact, it has been shown that as much as two third of the white matter contains at least two fiber bundles with different orientations (Behrens et al., 2007; Jeurissen et al., 2010). With fiber tractography, the direction of fiber bundles is followed from voxel to voxel, thus enabling the 3D reconstruction of white matter fiber bundles. By modeling more than one fiber direction per voxel, fiber tracking algorithms are better able to track through regions of the white matter with complex architecture.

\section{BRAIN NETWORKS}

Thanks to the recent developments made in $M R$ imaging and particularly computational image data analysis methods, it is now possible to measure how macroscopic brain regions are functionally connected and whether and how they are connected by white matter fiber bundles. Figure 1.4 illustrates the principles of what is imaged with the previously mentioned MRI techniques, functional MRI and Diffusion Weighted MRI. The in-vivo mapping of brain connections in human beings at a high spatial resolution was previously not possible. This opens up new opportunities for researches to investigate the properties of the human brain network, how they relate to cognitive functions, and how connectivity is affected by disease.

The term "network" has been used informally in brain research and in the first part of this chapter. But what do we mean by a network? Intuitively a network is a collection of entities that are somehow connected and thus the term network can be applied to a large number of systems like the brain, the internet, or groups of socially interacting humans. From the field of applied mathematics, a more formal definition of a network exists; here a network is often called a graph which is composed of its individual elements called the nodes and the links between nodes called the edges. A full description of the graph is given by its nodes and the existent edges and in some cases edge weights that quantify the strength of the link between the edges. Historically, the first use of a graph to solve a real world problem was that of the Seven Bridges of Königsberg (Euler, 1741; Stam et al., 2010). The city of Königsberg in Prussia (now Kaliningrad, Russia) was set on both sides of the Pregel River, and included two large islands which were connected to each other and the mainland by seven bridges. The problem was to find a walk through the city that would cross each bridge once and only once. This problem was solved by the famous mathematician Leonhard Euler in 1735, who reformulated the problem as a collection of nodes (representing the landmasses) and a set of edges (the bridges) and could thus show that no such walk existed (see Figure 1.5). With his solution he laid the foundation for modern graph theory.

The anatomical configuration of brain networks at multiple scales (from interneuronal connectivity to inter-regional connectivity) has long been the subject of much empirical neuroscience (Cajal et al., 1995; Swanson, 2011). However, only in the 
last decade, scientists have begun to explore the use of graph theoretical tools to study the configuration of brain networks (Bullmore and Sporns, 2009). This shift has primarily been made possible thanks to advantages made in theoretical networks sciences (Watts and Strogatz, 1998; Albert and Barabási, 2002; Girvan and Newman, 2002; Börner et al., 2007). Graph theoretical measures offer new and diverse ways to quantitatively characterize brain networks. The most widely known are the so called small-world parameters. A network has a small-world organization when it is highly clustered (e.g. group of nodes that share connections) and when the overall distance between nodes (the number of edges between any pair of nodes) is small. These properties can be quantified by the cluster coefficient $(C)$ and the characteristic path length $(L)$. There are many more graph parameters that can capture aspects of the network, such as modularity, node degree, and efficiency. Figure 1.6 shows a graphical presentation of several of these parameters. In graph terms, the brain can be thought of as a collection of nodes and edges at multiple scales. At the microscopic scale, individual neurons can be modeled as the nodes and axonal or dendritic projections can be the edges. At the mesoscopic scale nodes can be groups of neurons arranged in cortical columns and edges embody the functional or structural connection with other cortical columns. At the macroscopic scale the nodes of the brain graph relate to brain regions and the edges represent the functional connectivity between regions or the connection of white matter fiber bundles between regions. Micro- and mesoscopic scales remain difficult to measure noninvasively. The macroscopic scale is the one currently measurable with MRI. The question is now, what can graph theory of large-scale networks measured with MRI reveal about the organization of large-scale brain networks in health and disease?

In the last decade, researchers have begun to explore the topological properties of the structural and functional brain network at the macroscopic scale. It has been shown that the human brain network can indeed be regarded as small-world (Achard et al., 2006; Hagmann et al., 2008; Supekar et al., 2008; van den Heuvel et al., 2008; Bullmore and Sporns, 2009) and highly non-random (Sporns, 2011). Furthermore, several regions show exceptionally high connectivity (hub regions) (Achard et al., 2006; Hagmann et al., 2008; Iturria-Medina et al., 2008; Gong et al., 2009).

An important question in neuroscience is whether graph measures change in neurological disorders (Bassett and Bullmore, 2009). A growing body of literature is emerging, showing that the topological organization of large-scale brain networks is indeed abnormal in a variety of neurological and psychiatric disorders. Abnormal networks have been found in schizophrenia (Liu et al., 2008; Alexander-Bloch et al., 2010; van den Heuvel et al., 2010; Wang et al., 2011; Zalesky et al., 2011), Alzeimer's disease (Supekar et al., 2008; Buckner et al., 2009; Lo et al., 2010; Sanz-Arigita et al., 2010), Attention Deficit Hyperactivity Disorder (ADHD) (Wang et al., 2009), depression (Zhang et al., 2011a), stroke (Wang et al., 2010; Crofts et al., 2011) and even blindness (Shu et al., 2009). 
A
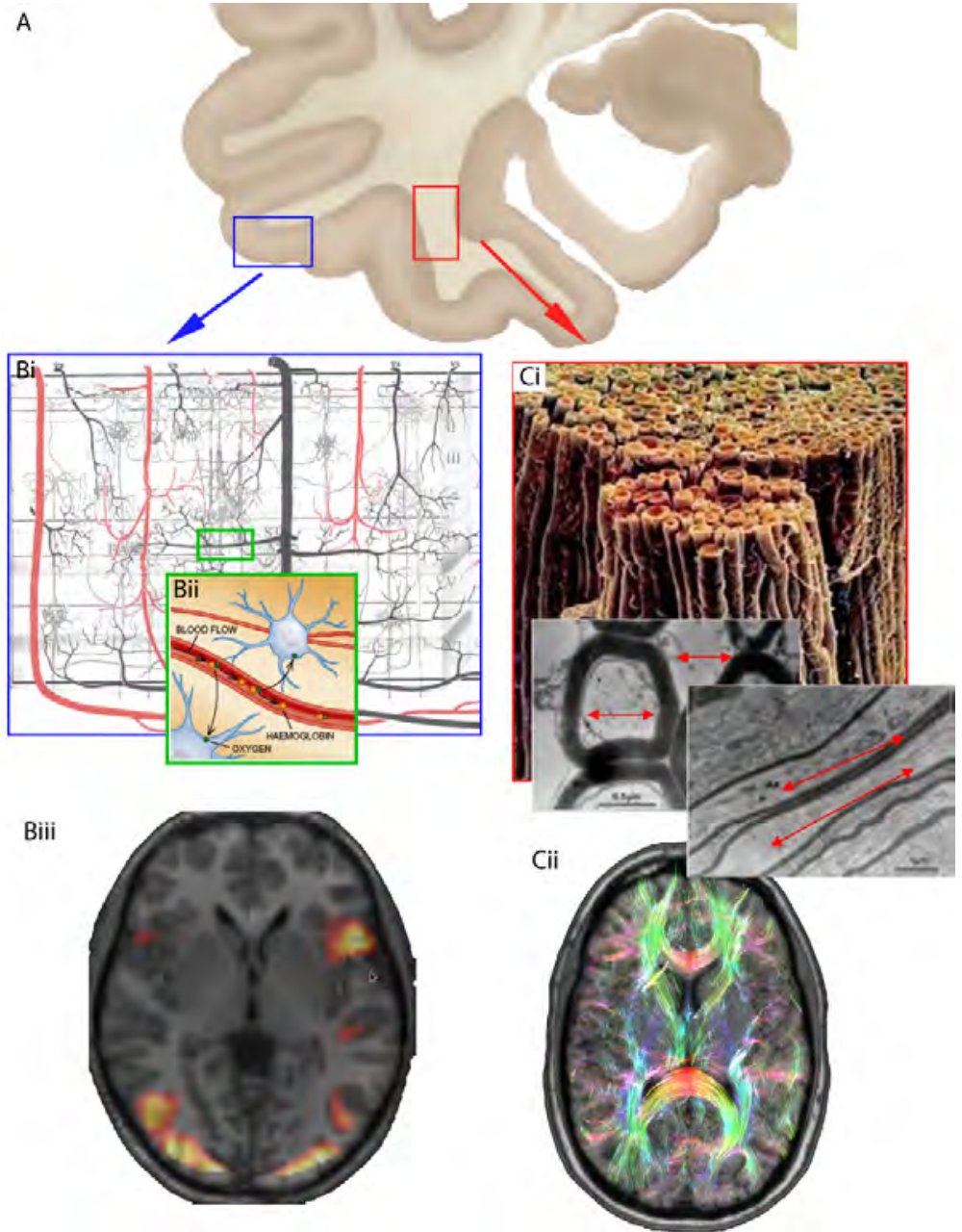

Biii

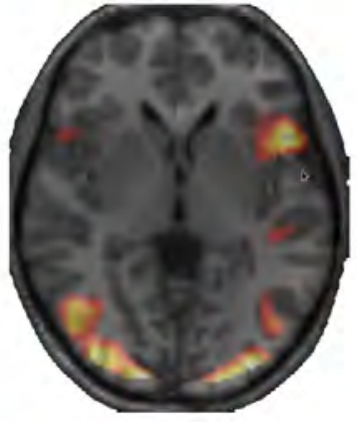

Figure 1.4 Biological mechanism behind functional and diffusion MRI. Panel (A) displayed a section of the human brain, where the gray (blue rectangle) and white (red rectangle) matter is clearly visible.

Left side: functional MRI (the BOLD signal) measures the physiological effects of neuronal activity. The cortex is highly perfused by blood vessels as shown in panel (Bi). When neurons become active, a mechanism is triggered that increases the blood flow to the activated region. This fresh blood contains oxyhaemoglobin, as oxygen consumption of neural tissue is smaller than the supply of fresh (oxygenated) blood, a relative decrease in deoxyhaemoglobin results (Bii). As de-oxyhaemoglobin is paramagnetic, it distorts the local magnetic field, and thereby the measured signal (phase coherence), a relative decrease in deoxyhaemoglobin causes an increase in MR signal intensity. Ther efore, in BOLD imaging, neural activity is associated with an increase in signal. Panel (Biii) displays and example of an activation map overlayed in color on a high-resolution anatomical scan.

Right side: The white matter of the adult human brain (red square in A) is organized in fiber bundles with highly coherent organization of myelinated axons (Ci). Water molecules can diffuse freely parallel to the axons but diffusion is hindered perpendicular to the axons (red arrows). Diffusion weighted imaging can be used to perform fiber tractography, as shown in panel (Cii). 
A

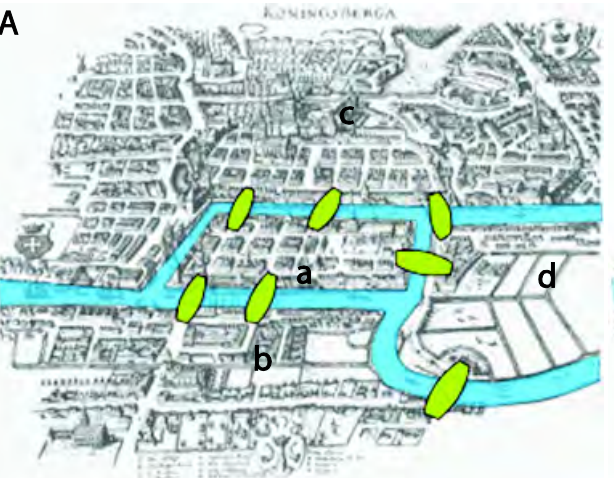

B

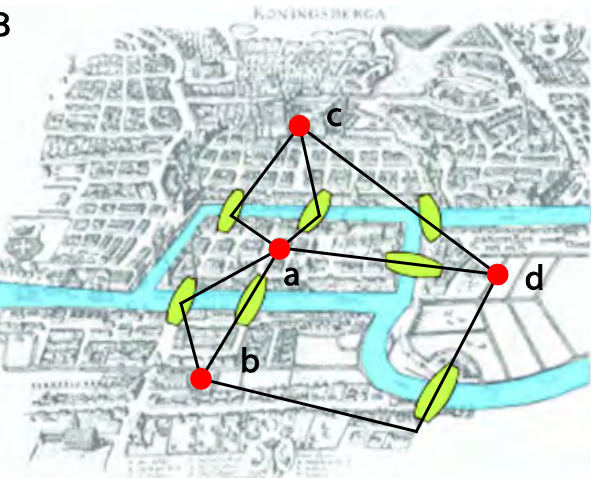

Figure 1.5 Konigsberg Bridges.(A) An antique map of Konigsberg with the river (blue) and bridges (green) examplified. (B) The graph representation of Konigsberg and its bridges. The red dots (nodes) relate to the landmasses and the black lines (edges) to the bridges.

\section{A. Regular}

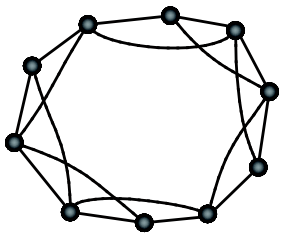

high C

high L
B. Small-world

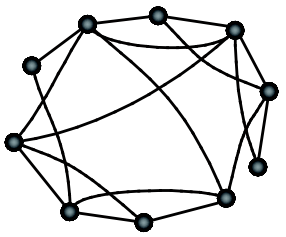

high C

low L
C. Random

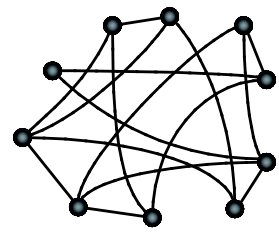

low C

low L

\section{Modular small-world}

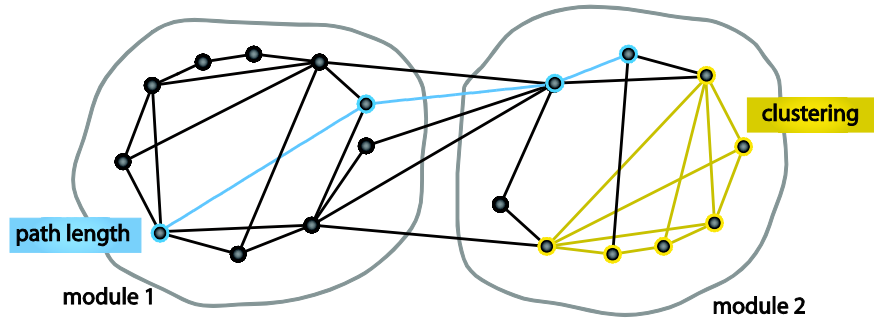

Figure 1.6 Graph theoretical measures. Different types of networks with distinct topology are shown. (A) A regular network (a.k.a. lattice network) consists of nodes that are only connected to their direct neighbors. As a consequence this type of network has a high clustering. A high path length arises from the fact that on average a large number of nodes has to be traversed to go from one node to another. (C) A network where all edges are placed at random is characterized by a low clustering; it is unlikely that highly interconnected nodes will emerge in a random setting. The path length of a random network is low, as many edges exist that connect different parts of the network. (B) The properties of high clustering and low path length are combined in a small-world network. A small-world network can be generated by adding a few 'long range' connections to a regular network. (D) A different concept that is not fully captured by clustering and path length is network modularity. This expresses the extent to which a network can be divided into modules (i.e. communities) that are highly interconnected but have little connectivity with the rest of the network. The concepts of clustering, path length and modularity are displayed as yellow edges, blue edges and gray outlines respectively in the bottom graph. 


\section{RATIONAL FOR NETWORK IMAGING OF IMPAIRED COGNITION}

As mentioned previously, one of the major complaints of patients with epilepsy are their cognitive problems. Abnormalities in the topological properties of brain networks have also been investigated for some types of epilepsy. Liao et al. (Liao et al., 2010) investigated functional networks in adult patients with temporal lobe epilepsy and found smaller clustering and shorter path length compared to healthy controls. A combination of functional and structural network analysis has also been performed in patients with idiopathic generalized epilepsy (Zhang et al., 2011b). However, the relation between cognitive impairment and the topological properties of brain networks in epilepsy has not been studied yet. Why would it be interesting to study this relation? First, cognitive functions are thought to rely on the orchestrated activity of a network of brain regions (Bressler and Menon, 2010; Deary et al., 2010). Furthermore, a relation between intelligence levels and graph theoretical measures has recently been shown (Li et al., 2009; van den Heuvel et al., 2009). Second, the expression of cognitive deficits in epilepsy is very heterogeneous (Braakman et al., 2011) and it is therefore unlikely that brain abnormalities will be localized at one specific location in the brain at the population level. Moreover, there is recent evidence that regions not directly affected by macroscopic lesions may show connectional abnormalities (He et al., 2007), as seen in stroke (Crofts et al., 2011) and epilepsy (Focke et al., 2008; Meador and Hermann, 2010). Taken together, these findings motivate an approach to studying cognition and epilepsy that is not targeted at finding localized abnormalities but rather focuses on abnormalities in large-scale networks.

\section{AIM AND OUTLINE OF THE THESIS}

The main goal of this thesis is to develop and explore the use of graph analysis methods for finding a neuronal correlate of cognitive impairment in epilepsy.

When the research for this thesis was started, the literature on applying graph analysis tools to large-scale brain networks was very limited (Sporns et al., 2005; Achard et al., 2006), and not much was known on the methodological issues that should be taken into consideration when constructing whole brain networks. Therefore, to gain insight into some of the factors that could influence brain network measures; in chapter 2 the effect of several imaging parameters on the reproducibility of graph metrics from networks constructed with fiber tractography is investigated.

As mentioned above, there are many different types of epilepsy. In this thesis, two different populations of patients are investigated and compared to healthy controls. Chapter 3 and 4 are based on the population from the CODICE (COgnitive 
Deterioration In Cryptogenic Epilepsy) study. In this study adult patients with localization related but cryptogenic epilepsy (i.e. no visible lesions on standard MRI) are included. Although all patients have a seizure onset in the frontal or temporal lobe, their epilepsy is of unknown origin. Chapters 5 and 6 are based on another cohort: the IMAGing In Epilepsy (IMAGINE) study. Here, children with localization related epilepsy, also without $M R$ visible lesions are included. All patients have a seizure focus in the frontal lobe.

Both these study populations have in common that the patients have localization related epilepsy, but normal appearing brains on standard imaging. As such, these patients are generally not candidates for epilepsy surgery when their seizures cannot be controlled by medication (refractoriness). At present, it is difficult for the clinician to give a prognosis to these patients: clinical variables such as age at onset of the epilepsy and seizure history do not provide reliable predictors for the long term outcome. Patients and relatives cannot be informed about whether cognition will remain intact and whether a certain anti-epileptic drug (AED) will successfully control seizures. Therefore, diagnostic tools to identify patients at risk of cognitive impairment and refractoriness are needed. Such tools could potentially be provided by biomarkers based on MR network imaging.

In chapter 3, measures of cognitive decline in patients with cryptogenic localizationrelated epilepsy are related to the topological properties of the entire functional cerebral network. In chapter 4, in the same patient cohort, these measures of cognitive decline are related to properties of the structural cerebral network. In chapter 5, graph theoretical measures of the modular organization of the entire cerebral network is investigated and related to cognitive variables in the same cohort of children with FLE. Finally, in chapter 6 , the relation between the functional and structural cerebral network properties are investigated in children with FLE. The sensitivity of the two modalities is explored together with the similarities and discrepancies of the two conceptually different connectivity measures. This thesis ends with a general discussion of the results and conclusions from the constituting chapters. 


\section{REFERENCES}

Achard S, Salvador R, Whitcher B, Suckling J, Bullmore E (2006) A resilient, low-frequency, small-world human brain functional network with highly connected association cortical hubs. J Neurosci 26:63-72.

Albert R, Barabási AL (2002) Statistical mechanics of complex networks. Reviews of modern physics 74:47.

Aldenkamp A, Arends J (2004) The relative influence of epileptic EEG discharges, short nonconvulsive seizures, and type of epilepsy on cognitive function. Epilepsia 45:54-63.

Alexander-Bloch AF, Gogtay N, Meunier D, Birn R, Clasen L, Lalonde F, Lenroot R, Giedd J, Bullmore ET (2010) Disrupted modularity and local connectivity of brain functional networks in childhoodonset schizophrenia. Front Syst Neurosci 4:147.

Alexander DC (2005) Multiple-fiber reconstruction algorithms for diffusion MRI. Ann N Y Acad Sci 1064:113133.

Baker GA, Jacoby A, Buck D, Stalgis C, Monnet D (1997) Quality of life of people with epilepsy: a European study. Epilepsia 38:353-362.

Bassett DS, Bullmore ET (2009) Human brain networks in health and disease. Curr Opin Neurol 22:340-347.

Behrens TE, Berg HJ, Jbabdi S, Rushworth MF, Wool rich MW (2007) Probabilistic diffusion tractography with multiple fibre orientations: What can we gain? Neuroimage 34:144-155.

Belliveau JW, Kennedy DN, Jr., McKinstry RC, Buchbinder BR, Weisskoff RM, Cohen MS, Vevea JM, Brady TJ, Rosen BR (1991) Functional mapping of the human visual cortex by magnetic resonance imaging. Science 254:716-719.

Berg AT, Berkovic SF, Brodie MJ, Buchhalter J, Cross JH, van Emde Boas W, Engel J, French J, Glauser TA, Mathern GW, Moshe SL, Nordli D, Plouin P, Scheffer IE (2010) Revised ter minology and concepts for organization of seizures and epilepsies: report of the ILAE Commission on Classification and Terminology, 2005-2009. Epilepsia 51:676-685.

Biswal B, Yetkin FZ, Haughton VM, Hyde JS (1995) Functional connectivity in the motor cortex of resting human brain using echo-planar MRI. Magn Reson Med 34:537-541.

Blume WT, Luders HO, Mizrahi E, Tassinari C, van Emde Boas W, Engel J, Jr. (2001) Glossary of descriptive terminology for ictal semiology: report of the ILAE task force on classification and terminology. Epilepsia 42:1212-1218.

Börner K, Sanyal S, Vespignani A (2007) Network science. Annual review of information science and technology 41:537-607.

Braakman HM, Vaessen MJ, Hofman PA, Debeij-van Hall MH, Backes WH, Vles JS, Aldenkamp AP (2011) Cognitive and behavioral complications of frontal lobe epilepsy in children: A review of the literature. Epilepsia 52:849-856.

Bressler SL, Menon V (2010) Large-scale brain networks in cognition: emerging methods and principles. Trends in cognitive sciences 14:277-290.

Brown GG, Perthen JE, Liu TT, Buxton RB (2007) A primer on functional magnetic resonance imaging. Neuropsychol Rev 17:107-125.

Buckner RL, Sepulcre J, Talukdar T, Krienen FM, Liu H, Hedden T, Andrews-Hanna JR, Sperling RA, Johnson KA (2009) Cortical hubs revealed by intrinsic functional connectivity: mapping, assessment of stability, and relation to Alzheimer's disease. J Neurosci 29:1860-1873.

Bullmore E, Sporns O (2009) Complex brain networks: graph theoretical analysis of structural and functional systems. Nat Rev Neurosci 10:186-198. 
Cajal SR, Swanson N, Swanson LW (1995) Histology of the nervous system of man and vertebrates: Oxford University Press.

Chaplin JE, Yepez Lasso R, Shorvon SD, Floyd M (1992) National general practice study of epilepsy: the social and psychological effects of a recent diagnosis of epilepsy. Bmj 304:1416-1418.

Crofts JJ, Higham DJ, Bosnell R, Jbabdi S, Matthews PM, Behrens TE, Johansen-Berg H (2011) Network analysis detects changes in the contralesional hemisphere following stroke. Neuroimage 54:161169.

Damasio H, Damasio AR (1989) Lesion analysis in neuropsychology: Oxford University Press.

Damoiseaux JS, Rombouts SA, Barkhof F, Scheltens P, Stam CJ, Smith SM, Beckmann CF (2006) Consistent resting-state networks across heal thy subjects. Proc Nat Acad Sci U S A 103:13848-13853.

Deary IJ, Penke L, Johnson W (2010) The neuroscience of human intelligence differences. Nat Rev Neurosci 11:201-211.

Dodrill CB (2002) Progressive cognitive decline in adolescents and adults with epilepsy. Prog Brain Res 135:399-407.

Euler L (1741) Solutio problematis ad geometriam situs pertinentis. Commentarii academiae scientiarum Petropolitanae 8:128-140.

Fisher RS, Vickrey BG, Gibson P, Hermann B, Penovich P, Scherer A, Walker S (2000) The impact of epilepsy from the patient's perspective I. Descriptions and subjective perceptions. Epilepsy Res 41:39-51.

Fisher RS, van Emde Boas W, Blume W, Elger C, Genton P, Lee P, Engel J, Jr. (2005) Epileptic seizures and epilepsy: definitions proposed by the International League Against Epilepsy (ILAE) and the International Bureau for Epilepsy (IBE). Epilepsia 46:470-472.

Focke NK, Yogarajah M, Bonelli SB, Bartlett PA, Symms MR, Duncan JS (2008) Voxel-based diffusion tensor imaging in patients with mesial temporal lobe epilepsy and hippocampal sclerosis. Neuroimage 40:728-737.

Fox MD, Raichle ME (2007) Spontaneous fluctuations in brain activity observed with functional magnetic resonanceimaging. Nat Rev Neurosci 8:700-711.

Fox PT, Lancaster JL (2002) Opinion: Mapping context and content: the BrainMap model. Nat Rev Neurosci 3:319-321.

Friston K (1994) Functional and effective connectivity in neuroimaging: A synthesis. Hum Brain Mapp 2:5678

Geschwind N (1965a) Disconnexion syndromes in animals and man. II. Brain 88:585-644.

Geschwind N (1965b) Disconnexion syndromes in animals and man. I. Brain 88:237-294.

Girvan M, Newman ME (2002) Community structure in social and biological networks. Proc Natt Acad Sci U S A 99:7821-7826.

Gong G, He Y, Concha L, Lebel C, Gross DW, Evans AC, Beaulieu C (2009) Mapping anatomical connectivity patterns of human cerebral cortex using in vivo diffusion tensor imaging tractography. Cerebral cortex 19:524-536.

Haas LF (2003) Hans Berger (1873-1941), Richard Caton (1842-1926), and el ectroencephalography. J Neurol Neurosurg Psychiatry 74:9.

Hagmann P, Cammoun L, Gigandet X, Meuli R, Honey CJ, Wedeen VJ, Sporns O (2008) Mapping the structural core of human cerebral cortex. PLoS Biol 6:e159.

He BJ, Shulman GL, Snyder AZ, Corbetta M (2007) The role of impaired neuronal communication in neurological disorders. Curr Opin Neurol 20:655-660.

He BJ, Snyder AZ, Zempel JM, Smyth MD, Raichle ME (2008) Electrophysiological correlates of the brain's intrinsic large-scale functional architecture. Proc Natl Acad Sci U S A 105:16039-16044. 
Hermann B, Jones J, Sheth R, Dow C, Koehn M, Seidenberg M (2006) Children with new-onset epilepsy: neuropsychological status and brain structure. Brain 129:2609-2619.

Hermann B, Seidenberg M, Bell B, Rutecki P, Sheth R, Ruggles K, Wendt G, O'Leary D, Magnotta V (2002) The neurodevelopmental impact of childhood-onset temporal lobe epilepsy on brain structure and function. Epilepsia 43:1062-1071.

Honey CJ, Kotter R, Breakspear M, Sporns O (2007) Network structure of cerebral cortex shapes functional connectivity on multiple time scales. Proc Natl Acad Sci U S A 104:10240-10245.

ILAE (1989) Proposal for revised classification of epilepsies and epileptic syndromes. Commission on Classification and Terminology of the International League Against Epilepsy. Epilepsia 30:389399.

Iturria-Medina Y, Sotero RC, Canales-Rodriguez EJ, Aleman-Gomez Y, Melie-Garcia L (2008) Studying the human brain anatomical network via diffusion-weighted MRI and Graph Theory. Neuroimage 40:1064-1076.

Jeurissen B, Leemans A, Tournier J, Jones D, Sijbers J (2010) Estimating the number of fiber orientations in diffusion MRI voxels: a constrained spherical deconvolution study. International Society for Magnetic Resonance in Medicine, Stockholm, Sweden.

Kuzniecky RI, Jackson GD (2005) Magnetic resonance in epilepsy: neuroimaging techniques: Elsevier Academic Press.

Kwong KK, Belliveau JW, Chesler DA, Goldberg IE, Weisskoff RM, Poncelet BP, Kennedy DN, Hoppel BE, Cohen MS, Turner R, et al. (1992) Dynamic magnetic resonance imaging of human brain activity during primary sensory stimulation. Proc Natl Acad Sci U S A 89:5675-5679.

Le Bihan D, Mangin JF, Poupon C, Clark CA, Pappata S, Molko N, Chabriat H (2001) Diffusion tensor imaging: concepts and applications. J Magn Reson Imaging 13:534-546.

Li Y, Liu Y, Li J, Qin W, Li K, Yu C, Jiang T (2009) Brain anatomical network and intelligence. PLoS computational biology 5:e1000395.

Liao W, Zhang Z, Pan Z, Mantini D, Ding J, Duan X, Luo C, Lu G, Chen H (2010) Alter ed functional connectivity and small-world in mesial temporal lobe epilepsy. PLoS One 5:e8525.

Liu Y, Liang M, Zhou Y, He Y, Hao Y, Song M, Yu C, Liu H, Liu Z, Jiang T (2008) Disrupted small-world networks in schizophrenia. Brain 131:945-961.

Lo CY, Wang PN, Chou KH, Wang J, He Y, Lin CP (2010) Diffusion tensor tractography reveals abnormal topological organization in structural cortical networks in Alzheimer's disease. J Neurosci 30:16876-16885.

Luria AR (1976) The working brain: an introduction to neuropsychology: Basic Books.

Meador KJ, Hermann B (2010) How localized is localization-related epilepsy? Neurology 75:386.

NEF (2009) Nationaal Epilepsie Fonds, www.epilepsie.nl.

Niedermeyer E (1997) Alpha rhythms as physiological and abnormal phenomena. Int J Psychophysiol 26:3149.

Ogawa S, Lee TM, Kay AR, Tank DW (1990) Brain magnetic resonance imaging with contrast dependent on blood oxygenation. Proc Natl Acad Sci U S A 87:9868-9872.

Panayiotopoulos CP (2002) A clinical guide to epileptic syndromes and their treatment. 1st ed. Oxford: Bladon Medical Publishing.

Parker GJ, Haroon HA, Wheeler-Kingshott CA (2003) A framework for a streamline-based probabilistic index of connectivity (PICo) using a structural interpretation of MRI diffusion measurements. Journal of magnetic resonance imaging : JMRI 18:242-254.

Salmenpera TM, Duncan JS (2005) Imaging in epilepsy. J Neurol Neurosurg Psychiatry 76 Suppl 3:iii 2-iii10. 
Sanz-Arigita EJ, Schoonheim MM, Damoiseaux JS, Rombouts SA, Maris E, Barkhof F, Scheltens P, Stam CJ (2010) Loss of 'small-world' networks in Alzheimer's disease: graph analysis of FMRI resting-state functional connectivity. PLoS One $5:$ e13788.

Shmuel A, Leopold DA (2008) Neuronal correlates of spontaneous fluctuations in fMRI signals in monkey visual cortex: Implications for functional connectivity at rest. Hum Brain Mapp 29:751-761.

Shu N, Liu Y, Li J, Li Y, Yu C, Jiang T (2009) Altered anatomical network in early blindness revealed by diffusion tensor tractography. PLoS One 4:e7228.

Smith SM, Fox PT, Miller KL, Glahn DC, Fox PM, Mackay CE, Filippini N, Watkins KE, Toro R, Laird AR, Beckmann CF (2009) Correspondence of the brain's functional architecture during activation and rest. Proc Natl Acad Sci U S A 106:13040-13045.

Sporns O (2011) The non-random brain: efficiency, economy, and complex dynamics. Front Comput Neurosci 5:5.

Sporns O, Tononi G, Kotter R (2005) The human connectome: A structural description of the human brain. PLoS Comput Biol 1:e42.

Stam K, Douw L, Haan W (2010) Hersenweb: wat moderne netwerktheorie ons kan leren over de werking van de hersenen: Prometheus BV Vassallucci, Uitgeverij.

Stehling MK, Turner R, Mansfield P (1991) Echo-planar imaging: magnetic resonance imaging in a fraction of a second. Science 254:43-50.

Sundgren PC, Dong Q, Gomez-Hassan D, Mukherji SK, Maly P, Welsh R (2004) Diffusion tensor imaging of the brain: review of clinical applications. Neuroradiology 46:339-350.

Supekar K, Menon V, Rubin D, Musen M, Greicius MD (2008) Network analysis of intrinsic functional brain connectivity in Alzheimer's disease. PLoS Comput Biol 4:e1000100.

Swanson LW (2011) Brain Architecture: Understanding the Basic Plan: Oxford University Press.

Taylor J, Kolamunnage-Dona R, Marson AG, Smith PE, Aldenkamp AP, Baker GA (2010) Patients with epilepsy: cognitively compromised before the start of antiepileptic drug treatment? Epilepsia 51:48-56.

Tournier JD, Calamante F, Connelly A (2007) Robust determination of the fibre orientation distribution in diffusion MRI: non-negativity constrained super-resolved spherical deconvolution. Neurolmage 35:1459-1472.

Tsao J (2010) Ultrafast imaging: principles, pitfalls, solutions, and applications. J Magn Reson Imaging 32:252-266.

Turner R, Le Bihan D, Moonen CT, Despres D, Frank J (1991) Echo-planar time course MRI of cat brain oxygenation changes. Magn Reson Med 22:159-166.

Urbach H (2005) Imaging of the epilepsies. Eur Radiol 15:494-500.

van den Heuvel MP, Stam CJ, Boersma M, Hulshoff Pol HE (2008) Small-world and scale-free organization of voxel-based resting-state functional connectivity in the human brain. Neuroimage 43:528-539.

van den Heuvel MP, Stam CJ, Kahn RS, Hulshoff Pol HE (2009) Efficiency of functional brain networks and intellectual performance. J Neurosci 29:7619-7624.

van den Heuvel MP, Mandl RC, Stam CJ, Kahn RS, Hulshoff Pol HE (2010) Aberrant frontal and temporal complex network structure in schizophrenia: a graph theoretical analysis. The Journal of neuroscience : the official journal of the Society for Neuroscience 30:15915-15926.

Vermeulen J, Aldenkamp AP (1995) Cognitive side-effects of chronic antiepileptic drug treatment: a review of 25 years of research. Epilepsy Res 22:65-95.

Vlooswijk MC, Jansen JF, de Krom MC, Majoie HM, Hofman PA, Backes WH, Aldenkamp AP (2010) Functional MRI in chronic epilepsy: associations with cognitive impairment. Lancet neurology 9:1018-1027. 
Wang L, Zhu C, He Y, Zang Y, Cao Q, Zhang H, Zhong Q, Wang Y (2009) Altered small-world brain functional networks in children with attention-deficit/hyperactivity disorder. Hum Brain Mapp 30:638-649.

Wang L, Yu C, Chen H, Qin W, He Y, Fan F, Zhang Y, Wang M, Li K, Zang Y, Woodward TS, Zhu C (2010) Dynamic functional reorganization of the motor execution network after stroke. Brain 133:12241238.

Wang Q, Su TP, Zhou Y, Chou KH, Chen IY, Jiang T, Lin CP (2011) Anatomical insights into disrupted smallworld networks in schizophrenia. Neuroimage.

Watts DJ, Strogatz SH (1998) Collective dynamics of 'small-world'networks. Nature 393:440-442.

Yogarajah M, Duncan JS (2008) Diffusion-based magnetic resonance imaging and tractography in epilepsy. Epilepsia 49:189-200.

Zalesky A, Fornito A, Seal ML, Cocchi L, Westin CF, Bullmore ET, Egan GF, Pantel is C (2011) Disrupted axonal fiber connectivity in schizophrenia. Biol Psychiatry 69:80-89.

Zhang J, Wang J, Wu Q, Kuang W, Huang X, He Y, Gong Q (2011a) Disrupted brain connectivity networks in drug-naive, first-episode major depressive disorder. Biol Psychiatry 70:334-342.

Zhang Z, Liao W, Chen H, Mantini D, Ding JR, Xu Q, Wang Z, Yuan C, Chen G, Jiao Q, Lu G (2011b) Altered functional-structural coupling of large-scale brain networks in idiopathic generalized epilepsy. Brain : a journal of neurology 134:2912-2928. 
28 Chapter 1 


\section{CHAPTER}

The effect and reproducibility of different clinical DTI gradient sets on small world brain connectivity measures

M.J. Vaessen, P.A.M. Hofman, R.H. Tijssen, A.P. Aldenkamp, J.F.A. Jansen, W.H. Backes Neurolmage 2010; 2010: 51(3):1106-16 


\section{ABSTRACT}

Advances in computational network analysis have enabled the characterization of topological properties in large scale networks including the human brain. Information on structural networks in the brain can be obtained in-vivo by performing tractography on diffusion tensor imaging (DTI) data. However, little is known about the reproducibility of network properties derived from whole brain tractography data, which has important consequences for minimally detectable abnormalities or changes over time. Moreover, acquisition parameters, such as the number of gradient directions and gradient strength, possibly influence network metrics and the corresponding reproducibility derived from tractography data. The aim of the present study is twofold: (i) to determine the effect of several clinically available DTI sampling schemes, differing in number of gradient directions and gradient amplitude, on smallworld metrics and (ii) to evaluate the interscan reproducibility of small-world metrics. DTI experiments were conducted on six healthy volunteers scanned twice. Probabilistic tractography was performed to reconstruct structural connections between regions defined from an anatomical atlas. The observed reproducibility of the network measures was high, reflected by low values for the Coefficient of Variation $(<3.8 \%)$, advocating the use of graph theoretical measurements to study neurological diseases. Small-world metrics were dependent on the choice of DTI gradient scheme and showed stronger connectivity with increasing directional resolution. The interscan reproducibility was not dependent on the gradient scheme. These findings should be considered when comparing results across studies using different gradient schemes or designing new studies. 


\section{INTRODUCTION}

Recently it has been shown that the topology of the structural network is linked to the dynamic behavior (or functional connectivity) of the brain (Honey et al., 2007; Park et al., 2008; Greicius et al., 2009). It is therefore interesting to study structural connectivity in the brain and relate it to functional connectivity, which might be reflected in behavioral data. For instance, it has recently been shown that topological properties of structural brain networks are related to intelligence (Li et al., 2009). Topological properties of large scale networks, including the human brain, can be characterized using methods from computational network analysis. One popular method in network analysis is the small-world model (Watts and Strogatz, 1998). Small-world networks are characterized by a topology in which most nodes are not neighbors of each other, but can be reached through a small number of steps. Recent studies have revealed that brain networks may possess small-world attributes (Sporns and Zwi, 2004; Bassett and Bullmore, 2006; Stam and Reijneveld, 2007; Hagmann et al., 2008). These attributes may be used to characterize the overall integrity of brain networks and may thus serve as clinical markers for several pathologies (Liu et al., 2008; Supekar et al., 2008).

Information on structural networks in the brain can be obtained in-vivo, by acquiring diffusion tensor imaging (DTI) data and subsequently performing tractography. DTI is an MRI technique which enables the measurement of water diffusion. In the brain, the movement of extra-cellular water molecules is hindered by cellular barriers present in biological tissue such as myelinated axons. Due to directional differences in water diffusion in different structures, DTI is able to provide information on the orientation of white matter (Le Bihan et al., 2001). Quantitative values derived from DTI data such as mean diffusivity (MD) or fractional anisotropy (FA) can provide valuable clinical information on local abnormalities related to various pathologies including Alzheimer's disease (Sundgren et al., 2004) and epilepsy (Yogarajah and Duncan, 2008).

Although DTI can provide parameters such as MD and FA, which yield information on the architecture of brain tissue at the voxel level, these parameters do not provide information on the projection of nerve fiber bundles between cerebral areas. Recently, a number of techniques have been developed to investigate the continuity of fiber orientations from voxel to voxel (e.g. streamline tractography). In this approach, the neuronal fiber orientation is assumed to be collinear with the principal direction of the diffusion tensor. However, this relationship is anatomically ambiguous and these methods are strongly affected by noise. Probabilistic tractography is an extension of streamline tractography that exploits the inherent uncertainty in principle diffusion direction to calculate the probability of connection from a seed voxel to other voxels in the brain (Behrens et al., 2003; Parker et al., 2003). This method is more robust to noise and is better able to cope with issues such as merging branching and dividing fiber bundles. 
Tractography studies can reveal localized network abnormalities by investigating one or more specific white matter tracts, whose existence and location are often supported by evidence from post mortem dissection or tracer studies (Bridge et al., 2008; Rilling et al., 2008). For this approach, accurate localization and quantification of the white matter pathways under investigation are important. However, in some pathologies such as epilepsy (Powell et al., 2007), Alzheimer (Supekar et al., 2008) and schizophrenia (Liu et al., 2008), the impairment does not necessarily reflect an abnormality of a single set of white matter tracts, and the exact location of the abnormality might be unknown. Since individual analysis of a large number of tracts is very impractical, a different type of analysis is needed where the integrity of the entire brain network can be assessed and quantified. This is where computational network analysis can play a critical role. However, little is known about the accuracy and reproducibility of network properties derived from tractography data, which has important consequences for the assessment of minimally detectable abnormalities or changes over time. Moreover, acquisition parameters, such as the number of gradient directions and the gradient strength, possibly influence network metrics and the corresponding accuracy derived from tractography data. As patient burden in terms of scan time is often an important aspect in clinical research, it is important to investigate clinically available DTI protocols with acceptable scan times.

In the process from DTI acquisition to the quantification of whole brain network metrics a large number of intermediate steps is involved. Each step adds to the variability of the outcome measures. A full characterization of the variability and reproducibility of the whole pipeline from image acquisition to network quantification requires for each step a careful analysis of the different possible settings, strategies and resulting parameters. Figure 2.1 schematically illustrates a number of the relevant steps and the sources of variation. In the current study, we investigated to which extent variations in image data acquisition, including diffusion gradient and test-retest variations, affect the resulting network metrics.

The aim of the present study is twofold: (i) to determine the effect of several clinically available DTI sampling schemes, differing in number of gradient directions and gradient amplitude, on small-world metrics and (ii), to evaluate the interscan reproducibility of small-world metrics that can be derived from whole brain structural connectivity data. 


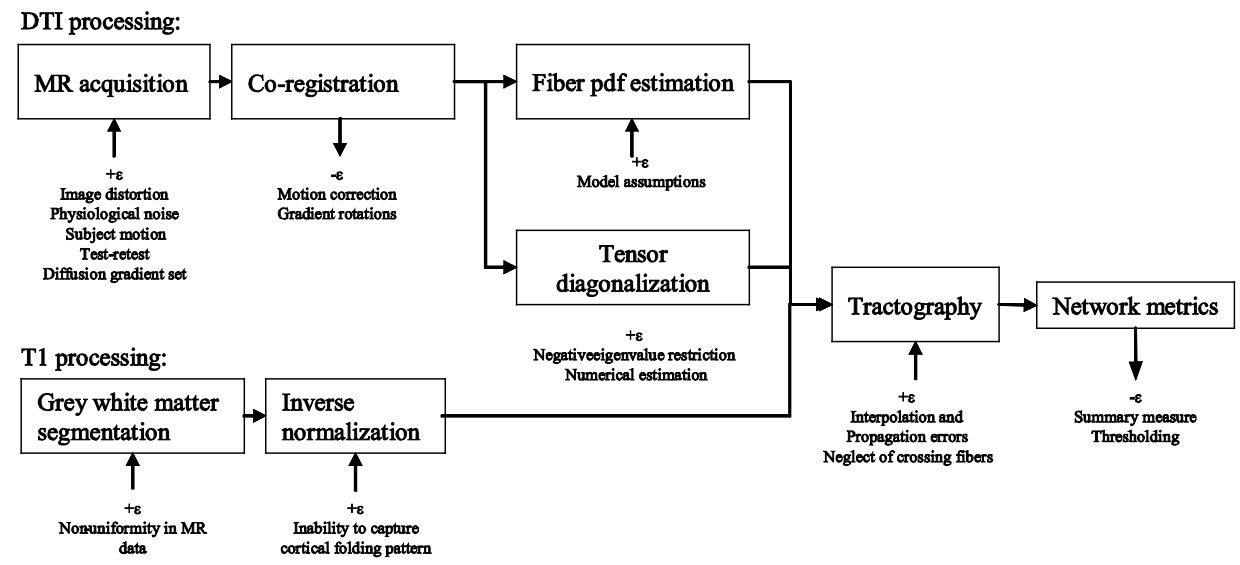

Figure 2.1 Sources of variation in the diffusion tensor image acquisition and processing pipeline. Diffusion tensor imaging is subject to a large number of sources of variation, including testretest variations, image distortion due to strong diffusion gradients and EPI acquisition, noise, and body motion. Part of the noise is reduced by image co-registration, which also partially corrects for eddy current distortions and motion.

Acquisition and processing steps for the T1-weighted image give rise to additional sources of variation, due to imperfect data and shortcomings of the segmentation and normalization methods. As a result, individual differences in brain anatomy might not be captured properly, leading to potential inaccurate placement of ROIs. Estimation of the fiber orientation PDF is based on numerical estimation and several modeling assumptions, possibly leading to a poor fit of the data. Tracking algorithm characteristics, such as spatial interpolation, and noise at the voxel level accumulate to some extent in the iterative steps of the tracking algorithm, further increasing possible errors.

Global network analysis captures properties of large scale networks in a small number of parameters, these summary measures appear to be less sensitive to errors from previous levels than more regional measurements.

\section{METHODS}

\section{Data acquisition}

DTI experiments were conducted in six healthy volunteers ( 5 male, 1 female, aged 23 28 years) , as previously described by Tijssen et al. (Tijssen et al., 2008). Every subject was scanned twice on different days, with an average interval between the scan sessions of $14 \pm 8$ days. Subjects signed informed consent prior to participation.

Each scan session consisted of a series of DTI measurements in which six sampling schemes were employed in randomized order. The six sampling schemes varied in number of diffusion directions ( $N$ dir $=32,15$ and 6 ) and gradient strength (unit-sphere or overplus). By combining the available gradient strength from each of the physical gradient axes of the scanner, the overplus schemes can employ stronger gradient strengths, which enables a shorter echo time (TE), and a higher signal-to-noise ratio (SNR). 
Acquisitions were performed on a 3T whole body scanner, release 2.0 (Philips Achieva, Philips Medical Systems, Best, the Netherlands) using a body coil for RF transmission and an 8-element SENSE head coil (SENSE-factor 2) for signal detection. The number of signal averages (NSA) was chosen such that the scan times of all the sampling schemes matched. All DTI datasets were obtained using a diffusion-weighted singleshot spin echo echo planar imaging (SE-EPI) sequence with a b-value of $800 \mathrm{~s} \cdot \mathrm{mm}^{-2}$. One $b=0 \mathrm{smm}^{-2}$ measurement per signal average was acquired for all sampling schemes. The echo time was $66 \mathrm{~ms}$ for the unit-sphere schemes and $56 \mathrm{~ms}$ for the overplus sampling schemes. The repetition time (TR) was set to $7600 \mathrm{~ms}$. All acquired images consisted of 52 contiguous axial slices, with a slice thickness of $2.5 \mathrm{~mm}$, a matrix size of $112 \times 112$, and a field of view set to $230 \times 230 \mathrm{~mm}$. Through interpolation, a matrix size of $128 \times 128$ and a final resolution of $1.8 \times 1.8 \times 2.5 \mathrm{~mm}^{3}$ were achieved. DTI parameters are summarized in Table 2.1.

Table 2.1 Differences in applied gradient schemes. Acquisition protocols, ' $t$ ' indicates that the 'overplus' setting was used. The 'overplus' gradient scheme combines available gradient strength from each of the physical gradient axes to achieve stronger gradient strength (Gamp) and thereby reducing echo time (TE). Number of gradient direction (Ndir) varied between 6 and 32.All schemes were matched for total scanning time (Tacq) by adjusting the number of signal averages (NSA). Condition numbers for the acquisition protocols were also calculated, lower condition number increases quality of the tensor estimation (Skare et al., 2000).

\begin{tabular}{lllllll}
\hline Gradient scheme & 6 & $6+$ & 15 & $15+$ & 32 & $32+$ \\
\hline Ndir & 6 & 6 & 15 & 15 & 32 & 32 \\
Gamp (mT/m) & 31 & 44 & 31 & 44 & 31 & 44 \\
TE (ms) & 66 & 56 & 66 & 56 & 66 & 56 \\
NSA & 14 & 14 & 6 & 6 & 3 & 3 \\
Tacq (min:s) & $13: 04$ & $13: 04$ & $12: 56$ & $12: 56$ & $14: 01$ & $14: 01$ \\
Condition number & 2.4 & 2.7 & 1.3 & 2.9 & 1.3 & 3.0 \\
\hline
\end{tabular}

\section{Data analysis}

The processing of the DTI data consisted of a number of steps: (1) combined motion correction of the diffusion weighted images and corresponding gradient rotations, (2) volume of interest (VOI) definition, (3) tractography from the defined VOls, and (4) post-processing of the generated tracts to derive quantitative tract measures and small-world metrics.

\section{Step 1: Motion and eddy current distortion correction}

Each data set was spatially co-registered to the $b=0$ image with an affine transformation to correct for head motion and eddy-current distortions utilizing CATNAP (Co-registration, Adjustment, and Tensor-solving, a Nicely Automated Program, version 1.3) (Farrell et al., 2007; Landman et al., 2007). Co-registration of 
images may correct for discrepancies between spatial orientations, but alters the original orientation relative to the diffusion direction. To correct for discrepancies between the relative orientation of the DW images and the diffusion gradient, the set of gradient vectors was adjusted according to the rotation of the individual images, as implemented in the CATNAP software.

\section{Step 2: VOI definition}

Only voxels on the boundary of the grey-white matter interface were used for initiating tractography. Selecting only voxels on the grey-white matter interface (with a relatively high FA value), reduces the number of false positives in the tractography results, since grey matter voxels usually yield unreliable tracts. The grey-white matter boundary was defined by first performing a probabilistic tissue segmentation on the subjects' T1-weighted images (FAST, FMRIB's Automated Segmentation Tool) and then selecting voxels where the joint tissue probability $(T)$ for grey and white matter was above a certain threshold ( $T>0.2)$. The results were transformed from the subjects' T1weighted image space to diffusion image space, using a rigid body transformation (FSL FLIRT (Smith et al., 2004)).

A brain atlas (WFUpick atlas (Maldjian et al., 2003)) was used to define all Brodmann areas (BA) in standard stereo taxis (MNI) space. The BAs were then transformed to DTI space of every individual, by applying a nonlinear transformation (SPM (Ashburner and Friston, 1999)). Next, each voxel in the grey-white matter boundary was labeled according to its shortest Euclidean distance to any of the Brodmann areas. This process is illustrated in Figure 2.2.

In this way, a VOI consisting of grey-white matter voxels labeled to the nearest Brodmann area, was obtained for each DTI data set, which is then used as input for the tractography.

\section{Step 3: Tractography}

Probabilistic tractography was performed in original DTI space according to previously described methods (Parker et al., 2003) using the CAMINO toolbox (Cook et al., 2006). The Probabilistic Index of Connectivity (PiCO) algorithm was used to track from the defined VOIs in the original space. This method models uncertainty, due to noise, in fiber orientation with probability density functions (PDFs). This method is based on streamline tractography, but incorporates Monte Carlo sampling methods to generate maps of connection probabilities from selected seed regions. One thousand tracts were generated for each seed voxel and tracts were terminated using a curvature threshold of $60^{\circ}$ (Toosy et al., 2004). Tractography was performed in original DTI space.

For all subjects, an individual cerebrum mask was created by applying the Brain Extraction Tool (BET (Smith, 2002)) on the $b=0$ diffusion image. This mask was used to limit the tractography to within the cerebrum. 
Tractography was initiated from all voxels in the grey-white matter VOI. Subsequently, cortico-cortical connections were calculated by counting the number of tracts reconstructed between all pairs of cortical areas.

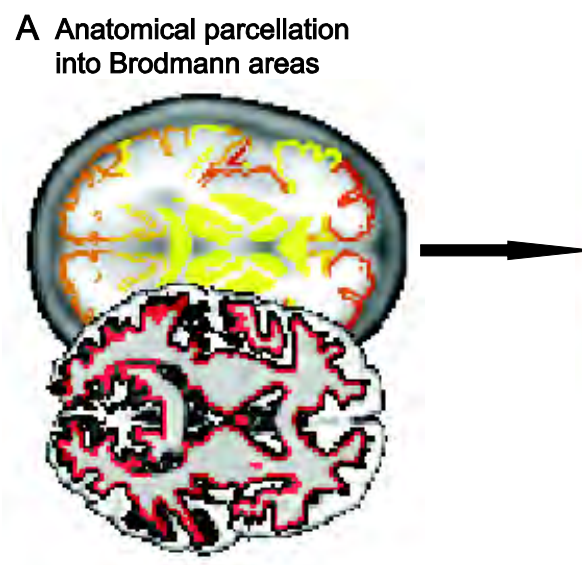

D Brain graph

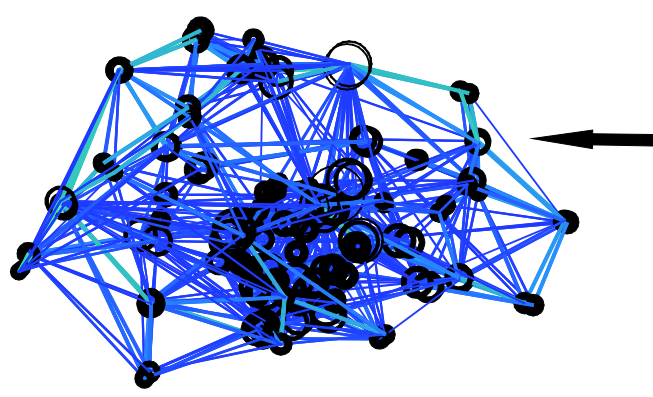

B Tractography

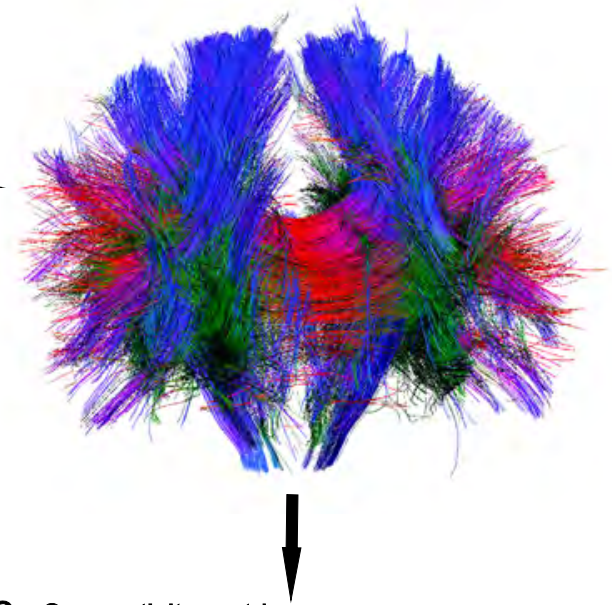

C Connectivity matrix

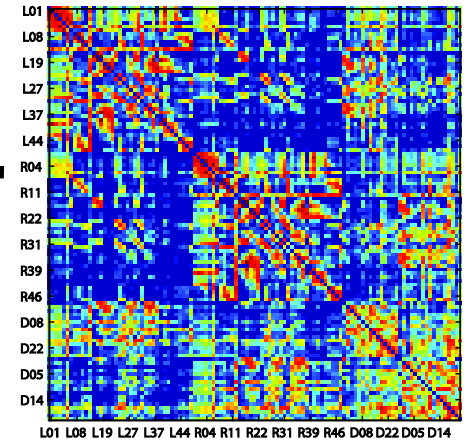

Figure 2.2 Preprocessing steps. (A) The grey-white matter interface (red) derived from a tissue segmentation of the T1-weighted image (bottom) is overlaid on an anatomical atlas containing the Brodmann areas (BA) (top). Both images are combined to construct an individual ROI containing the BAs mapped to the GM-WM interface. (B) Tractography is initiated from the GM-WM interface. (C) Resulting connections strengths between all pairs of brain regions are stored in the connection matrix (with the different BAs on the axes) which is subsequently thresholded to form the brain graph. The color temperature indicates the number of connections (hot is more connections). (D) Brain regions are displayed as black circles, circle size and thickness are scaled to node degree and cluster coefficient, respectively, of the corresponding brain region. Connections between brain regions are plotted as lines with a thickness increasing with number of fiber connections. 


\section{Step 4: Small world network analysis}

Computational networks measures were used to evaluate the cortical connections obtained from tractography (Watts and Strogatz, 1998; Strogatz, 2001; Sporns and Zwi, 2004; Bassett and Bullmore, 2006; Stam and Reijneveld, 2007; Gong et al., 2008). The connection strength between two areas $i$ and $j$ was calculated by counting the number of tracts originating from area $i$ and reaching area $j$. A binary connection matrix $A$ was formed by setting all elements (i.e. connections) where at least one tract was reconstructed to 1 and all others to 0 . The connection matrix $A$ is a numerical representation of a graph, which is an abstract data structure, consisting of nodes connected by edges. In the graph, a node is related to a brain region and is equal to a row or column from the connection matrix. An edge $e_{i, j}$ in the graph is a connection between brain areas $i$ and $j$, provided that $A_{i . j}>0$. The degree $k$ of node $i$ is the number of connections to other areas: $k_{i}=\sum_{j=i, j \neq i}^{N} A_{i, j}$, where $N$ is the total number of nodes (i.e. considered brain regions) in the graph. In the network analysis individual graphs were thresholded to create graphs with an equal number of nodes and edges (Stam et al., 2007). This was achieved by selecting the $T_{e}$ strongest connections (connections with the highest fiber count) and setting all other connections to zero. The threshold value was expressed as a sparsity value relating the number of edges maintained in the network to the total number of edges possible (Achard and Bullmore, 2007). Let $T_{e}$ be the number of edges maintained in the network, then the sparsity is defined as:

$$
\text { sparsity }=\left(N^{2}-N-T_{e}\right) /\left(N^{2}-N\right)
$$

The graph theoretical metrics mean node degree $(K)$, characteristic path length $(L)$ and cluster coefficient $(C)$ were calculated to perform analysis on the constructed brain graphs. Mean node degree is the average node degree over all nodes in the graph $(G)$, and is defined as:

$$
K=\frac{1}{N} \sum_{i} k_{i}
$$

Characteristic path length is defined as the average distance, in number of edges, connecting any two nodes in the graph:

$$
L=\frac{1}{N(N-1)} \sum_{i, j \in G, i \neq j} d_{i, j}
$$

where $d_{i . j}$ is the length of the shortest path between nodes $i$ and $j$. The characteristic path length is a measure of how well connected a network is. Small characteristic path length indicates an average short distance between any two nodes, i.e. they can be reached through a small number of steps. The cluster coefficient is defined as the number of actual edges connecting the neighbors of a node divided by the maximum number of edges possible between neighboring nodes,

$$
C=\frac{1}{N} \sum_{i} \frac{\sum_{j, m} A_{i, j} A_{j, m} A_{m, i}}{k_{i}\left(k_{i}-1\right)}
$$


The cluster coefficient of a network is a measure of how many local clusters exist in the network. A high cluster coefficient indicates that the neighbors of a node are often also directly connected to each other, i.e. they form a cluster.

To be able to determine whether a network has small-world properties, the values of $L$ and $C$ must be scaled to values from generated random networks (Watts and Strogatz, 1998). Small world networks are characterized by having characteristic path lengths that are similar to those of comparable random networks $\left(L_{\text {random }}\right)$ but with increased cluster coefficients $\left(C_{\text {random }}\right): \lambda=L / L_{\text {random }} \approx 1$ and $\gamma=C / C_{\text {random }}>$ 1 . The value $\sigma=\gamma / \lambda$ can be used to signify the 'small-worldness' of a network and is typically larger than 1 for small-world networks (Humphries et al., 2006). Random networks were generated by considering each existing edge in the original network between the nodes $i$ and $j, e_{i . j}$ and connecting it to another randomly chosen node $j 2$, with the condition that $e_{i, j 2}$ was not present in the original network (Maslov and Sneppen, 2002). This process ensures that the node degree and node distribution of the random network is similar to the original network.

We investigated both non-thresholded networks and thresholded networks. The nonthresholded networks do not necessarily have an equal number of edges between subjects and scans. As the number of edges, or similarly the node degree $K$, strongly influences the small-world metrics (Stam et al., 2007), these metrics can only be compared in a meaningful way when the number of edges is held constant over subjects and scans. For this reason the small-world metrics $L, C, \lambda, \gamma$ and $\sigma$ were only calculated for the thresholded networks as a function of the same number of edges. As the number of edges found in the networks might also provide useful information, we also analyzed the average node degree for the non-thresholded networks.

\section{Quantification of tract length}

A previous study (Tijssen et al., 2008) indicated that a lower number of gradient directions resulted in lower FA values for the white matter. This effect might also negatively affect the tractography results, especially tract length, as lower FA values and more randomly distributed principal diffusion directions can result in an earlier termination in the fiber tracking algorithm. To investigate this effect, the average tract length was computed for all gradient schemes. Termination of fibers can be caused by a low FA value $(F A<0.2)$, a tract curvature greater than a certain angle (threshold angle, $60^{\circ}$ ) or by reaching a voxel outside the cerebrum.

\section{Quantification of reproducibility}

To characterize the interscan reproducibility, three quantities were used: the Coefficient of Variation (CV), the Repeatability Coefficient (RC) and the Intra Class Correlation (ICC).

The CV used here is a pooled within group coefficient of variation (Lachin, 2004) and is defined as the mean within subject standard deviation $\left(\sigma_{w s}\right)$ divided by the overall 
measurement mean. The CV gives an indication of minimum percentage signal change detectable in repeated measures.

The RC is defined as $1.96 \cdot \sqrt{2} \cdot \sigma_{w s}$ (Bland and Altman, 1986), where $\sigma_{w s}$ is the withinsubject standard deviation. The RC represents the minimum detectable difference of a measurement method. The difference between two measurements of the same subject is expected to be less than the RC in $95 \%$ of the observations.

The ICC can be interpreted as the proportion of total variance accounted for by the between-subject variation (Lachin, 2004). The ICC is calculated as

$$
I C C=\frac{\sigma_{b s}^{2}}{\sigma_{b s}^{2}+\sigma_{w s}^{2}}
$$

where $\sigma_{b s}^{2}$ is the variance between subjects, and $\sigma_{w s}^{2}$ is the pooled variance within subjects.

\section{Statistical analysis}

The effect of gradient scheme on values of $K, L$ and $C$ and the reproducibility measures, were analyzed using a two-way ANOVA test with a Tukey HSD post hoc test in the commercial software application SPSS (version 16.0, SPSS Inc., Chicago, IL). Factors included were the number of directions (Ndir) and gradient strength (Gamp) of the applied gradient schemes.

\section{RESULTS}

\section{Atlas based parcellation}

A total of $N=111$ (out of the 150 regions available) regions of interest were mapped to the grey white matter interface, consisting of Brodmann areas and sub cortical structures as defined in the WFU pickatlas anatomical atlas. This yielded a network with a maximum number of edges equal to $N \cdot(N-1)=12210$. The threshold values $T_{e}$ ranged from 3323 to 1722 edges in 27 steps. This corresponds to a sparsity value ranging from 0.728 to 0.859 . These values were chosen such that $T_{e}$ was smaller than the lowest number of edges found in any of the networks (number of edges $=3383$ ) but not so low that any of the networks became disconnected (this occurs when one or more nodes are not connected to the rest of the network). VOI sizes (mean 226 voxels; range 12-1480 voxels) were consistent between the two different measurements in the same subject (a paired sample t-test between the VOI sizes of two different measurements showed no significant difference: $p=0.35$ ). 


\section{Tractography results}

To visualize the results of the basic tractography method, a combination of two BAs (BA 6 left - BA 19 right and BA 28 left - BA 10 left) were selected and the connecting tracts were calculated. The tractography results were averaged over all subjects and a maximum intensity plot was made (Figure 2.3).

A
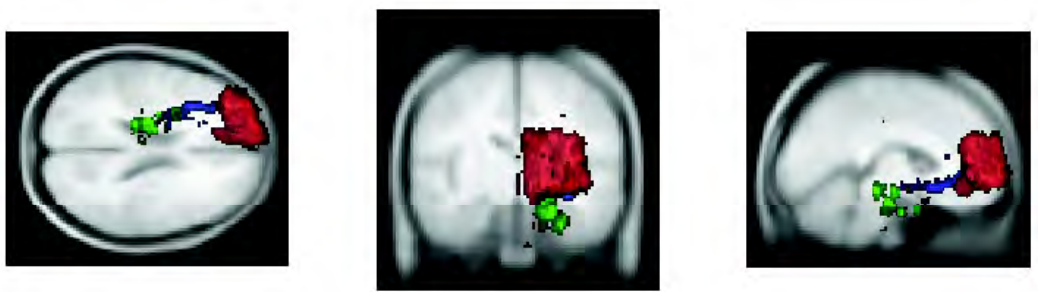

BA 28 Left (Entorhinal cortex, green) to BA 10 Left (red)

B
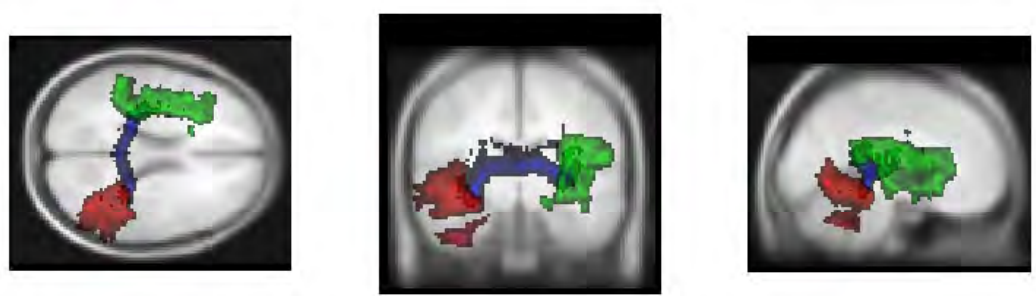

\section{BA 13 Left (green) to BA 37 Right (red)}

Figure 2.3 Tractography examples. (A) Maximum intensity projection rendering of fiber tracts (blue) connecting Brodmann areas BA 28 left connecting with BA 10 right, and (B) BA 13 left with BA 37 right. Displayed data is from the group average in $\mathrm{MNI}$ space over all gradient schemes. Only voxels where in at least $4 \%$ of the total number of available scans a tract was found are shown. The background image shows the MNI T1 template.

\section{Effect of gradient scheme on small world metrics}

\section{Non-thresholded networks}

For the non-thresholded networks, the average node degree $K$ increased significantly with directional resolution $(p<0.001)$.

In Figure 2.4 a histogram of the tract lengths is plotted. Lower directional resolution was associated with fewer long range tracts. Visual inspection revealed that the histograms of tract length mainly differed in the tails of the distributions, i.e. tract length $>40 \mathrm{~mm}$. To quantify these differences, the $80 \%$ quantile tract length value of the distribution was calculated. The $80 \%$ quantile is a certain tract length, such that $80 \%$ of all tract lengths are shorter than this $80 \%$ quantile. Results for the statistical analysis (ANOVA) of the quantiles are shown in Table 2.2. There was a significant effect (ANOVA) for both Ndir and Gamp on the quantiles $(p<0.001$ and $p<0.02$, respectively). 


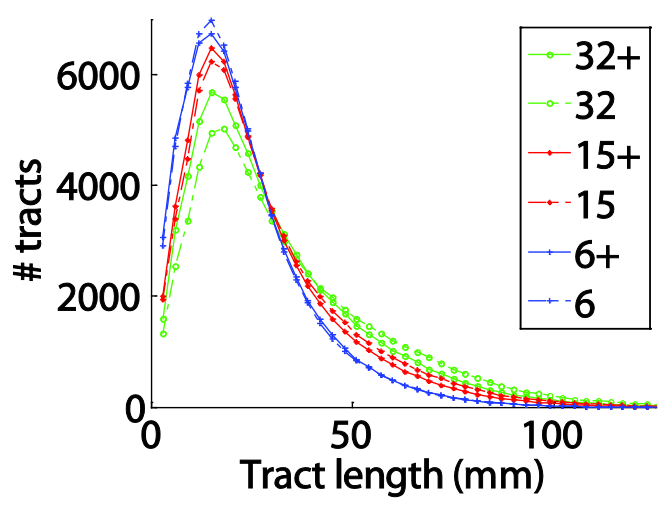

Figure 2.4 Histogram of tract lengths. Histogram distributions of the number of tracks plotted as function of the tract length for each scheme, averaged over all subjects. Low (6 directions) schemes are in blue, medium (15 directions) schemes in red, and high (32 directions) schemes are in green. Overplus schemes are indicated with continuous lines, whereas no-overplus schemes are indicated with broken lines. It can be appreciated that higher directional resolution is associated with more long tracts.
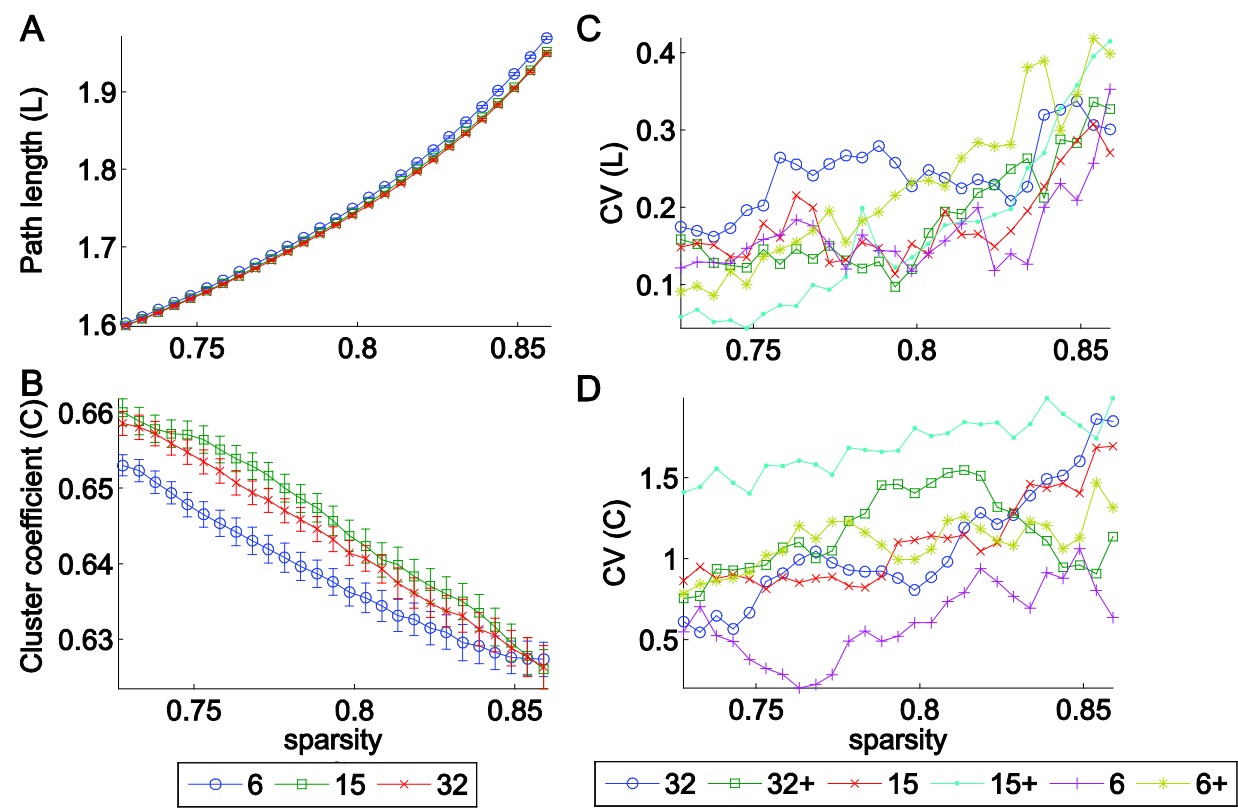

Figure 2.5 Small-world metrics as a function of gradient scheme. Graph the reproducibility (CV) of average path length $(L)$ and cluster coefficient $(C)$ over a range of sparsity values. Higher sparsity values indicate that more edges were removed from the network. (A) Higher sparsity values are associated with an increase in L. (B) A higher sparsity yields a decrease in $C$. (C) The CV of path length slightly increases with increasing sparsity, although CV values remain low. (D) The CV of $C$ increases somewhat as a function of sparsity, al though CV values remain low over all sparsity values. 
Table 2.2 The effect of gradient scheme on fiber tract length. ANOVA table for the effect of the number of gradient directions (Ndir) and gradient amplitude (Gamp) on the $80 \%$ quantile of tract length distribution. Both Ndir and Gamp had a significant effect on the tract length distribution.

\begin{tabular}{llll}
\hline & & $80 \%$ quantile & \\
\cline { 3 - 4 } & & & $p$-value \\
\hline Between subject effect & Ndir & & $<0.001$ \\
& Gamp & & 0.016 \\
& & Mean difference & $p$-value \\
Least significant difference Ndir & $6-15$ & -13.5 & $<0.001$ \\
& $6-32$ & -28.2 & $<0.001$ \\
& $15-32$ & -14.8 & $<0.001$ \\
\hline
\end{tabular}

\section{Thresholded networks}

The effect of gradient scheme was similar for the range of edge thresholds investigated, therefore we only report numerical values and statistical results of the small-world values for one representative threshold at sparsity $=0.74$. The full range of threshold values is presented in Figure 2.5. For $L$, the effect of number of gradient directions was significant over the whole range of sparsity values. The effect of number of gradient directions was significant for $C$ over a sparsity range of 0.73 to 0.76. Values for $\lambda, \gamma$ and $\sigma$ can be found in supplementary Figure S.2.1.

For a sparsity value of 0.74 , the cluster coefficient $C$ increased significantly $(p<0.005)$ with directional resolution (for 6 and 15 gradient directions and 6 and 32 gradient directions, no significant difference between 15 and 32 gradient direction was found). Characteristic path length $L$ showed significant decrease $(p<0.005)$ with directional resolution. Gradient amplitude did not have a significant effect on any of the metrics. The results of the statistical tests for this sparsity value are summarized in Table 2.3. All reconstructed networks showed small world properties when compared to randomly generated networks of the same size and average node degree. Characteristic path lengths were very close to those of random networks. The mean for all gradient schemes was $\lambda=1.020$ (range, 1.019-1.023). Cluster coefficients were much larger than those of random networks, with a mean $\gamma=2.28$ (range, 1.26-2.30).

\section{Reproducibility of small world metrics}

\section{Non-thresholded networks}

A summary of the reproducibility measures for all small world metrics can be found in Table 2.4. For average node degree $(K)$ CV was $<3.2 \%$ for all DTI gradient schemes, and $\mathrm{RC}$ was $<3.68$. Mean (standard deviation) of the ICC values for $K$ were $0.63(0.20)$. 
Table 2.3 The effect of gradient schemes on small-world metrics. Analysis of variance (ANOVA) assessing the effect of nu mber of gradient directions (Ndir) and gradient amplitude (Gamp) on small world metrics average node degree $(K)$, path length $(L)$ and cluster coefficient $(C)$. Ndir had a significant effect, while Gamp did not have a significant effect.

\begin{tabular}{|c|c|c|c|c|c|c|c|}
\hline \multirow{2}{*}{$\begin{array}{l}\text { Small world } \\
\text { metric }\end{array}$} & & \multicolumn{2}{|l|}{$K$} & \multicolumn{2}{|l|}{$L$} & \multicolumn{2}{|l|}{ C } \\
\hline & & & $p$-value & & $p$-value & & $p$-value \\
\hline Between & Ndir & & $<0.001$ & & $<0.005$ & & $<0.005$ \\
\hline \multirow[t]{2}{*}{ subject effect } & Gamp & & 0.2 & & 0.7 & & 0.8 \\
\hline & & $\begin{array}{l}\text { Mean } \\
\text { difference }\end{array}$ & $p$-value & $\begin{array}{l}\text { Mean } \\
\text { difference }\end{array}$ & $p$-value & $\begin{array}{l}\text { Mean } \\
\text { difference }\end{array}$ & $p$-value \\
\hline Least significant & $6-15$ & -6.175 & $<0.001$ & 0.003 & $<0.019$ & -0.007 & $<0.002$ \\
\hline \multirow[t]{2}{*}{ difference Ndir } & $6-32$ & -9.017 & $<0.001$ & 0.004 & $<0.002$ & -0.006 & $<0.012$ \\
\hline & $15-32$ & -2.843 & $<0.001$ & 0.01 & 0.436 & 0.001 & 0.568 \\
\hline
\end{tabular}

\section{Thresholded networks}

The CV of the network metrics was low over the whole range of thesholds as can be seen from Figure 2.5. Therefore we only report numerical values of reproducibility for one representative threshold at sparsity $=0.74$. Generally, CV values were low (smaller than $0.5 \%$ for $L$ and smaller than $1.9 \%$ for $C$ ), with a slight increase in CV values for increasing sparsity for both $L$ and $C$. Graphs for $C V$ of $\lambda, \gamma$ and $\sigma$ can be found in supplementary Figure S.2.1.

Average pathlength $(L)$ at sparsity $=0.74$ showed a $\mathrm{CV}<0.17 \%$ and an $\mathrm{RC}<0.01$, for all DTI gradient schemes. For the cluster coefficient $(C)$ CV was $<1.47 \%$ and $\mathrm{RC}$ was $<$ 0.03 . Mean (standard deviation) of the ICC values for $L$ and $C$, were $0.64(0.20)$ and 0.47 (0.31), respectively.

Both Ndir and Gamp did not have a significant effect on CV, RC and ICC values.

\section{Comparison of small world analysis with individual connections}

The reproducibility of tracts count (connection strength) from connections between brain regions was compared to reproducibility values of network metrics. In Figure 2.6, a histogram of $\mathrm{CV}$ values for connection strength of all pairs of brain regions (from the non-thresholded networks) is compared to the CV histogram of node degree and cluster coefficient of all brain regions. The CV for connection strength has a wide distribution and was generally larger than $10 \%$, indicating that most individual connections cannot be reliably reconstructed. CV values for node degree and cluster coefficient are much lower. Histograms of ICC values can be found in the supplementary Figure S.2.2.

In Figure 2.7, a Bland-Altmann plot is shown for the small world metrics and connection strength. In this plot, the agreement between two measurements in relation to their mean value can be appreciated. The scatter plots show that there is 
no obvious relation between measurement errors and effect size for any of the diffusion measures. That is, with an increased magnitude of the metrics, the measurement error does not significantly change.

Table 2.4 Reproducibility values of the small-world metrics. Mean, standard deviation (SD), coefficient of variation (CV), repeatability coefficient (RC), and intra class correlation (ICC) for small world metrics node degree $(K)$, characteristic path length $(L)$, and cluster coefficient $(C)$. Number of gradient directions (Ndir) is 6,15 or 32, ' + ' indicates the use of scan parameter 'overplus'.

\begin{tabular}{|c|c|c|c|c|c|}
\hline Ndir & mean & $S D$ & CV\% & $R C$ & ICC \\
\hline \multicolumn{6}{|c|}{$\bar{K}$ (Non-thresholded) } \\
\hline 32 & 44.65 & 2.17 & 2.81 & 3.46 & 0.70 \\
\hline $32+$ & 43.64 & 2.49 & 2.11 & 2.54 & 0.70 \\
\hline 15 & 41.91 & 2.17 & 2.19 & 2.54 & 0.77 \\
\hline $15+$ & 40.69 & 2.72 & 3.78 & 4.24 & 0.64 \\
\hline 6 & 35.03 & 1.57 & 1.44 & 1.39 & 0.23 \\
\hline $6+$ & 35.21 & 1.62 & 2.09 & 2.03 & 0.76 \\
\hline \multicolumn{6}{|c|}{$L($ Thresholded sparsity $=0.74)$} \\
\hline 32 & 1.6235 & 0.0054 & 0.25 & 0.0077 & 0.41 \\
\hline $32+$ & 1.6240 & 0.0042 & 0.14 & 0.0056 & 0.67 \\
\hline 15 & 1.6243 & 0.0049 & 0.17 & 0.0061 & 0.69 \\
\hline $15+$ & 1.6252 & 0.0043 & 0.07 & 0.0024 & 0.94 \\
\hline 6 & 1.6291 & 0.0030 & 0.13 & 0.0057 & 0.42 \\
\hline $6+$ & 1.6267 & 0.0032 & 0.12 & 0.0053 & 0.70 \\
\hline \multicolumn{6}{|c|}{$C($ Thresholded sparsity $=0.74)$} \\
\hline 32 & 0.6550 & 0.0069 & 0.68 & 0.0102 & 0.73 \\
\hline $32+$ & 0.6568 & 0.0084 & 1.12 & 0.0168 & 0.37 \\
\hline 15 & 0.6588 & 0.0088 & 1.03 & 0.0163 & 0.55 \\
\hline $15+$ & 0.6556 & 0.0091 & 1.55 & 0.0265 & 0.00 \\
\hline 6 & 0.6484 & 0.0048 & 0.53 & 0.0087 & 0.88 \\
\hline $6+$ & 0.6510 & 0.0084 & 1.09 & 0.0158 & 0.35 \\
\hline
\end{tabular}



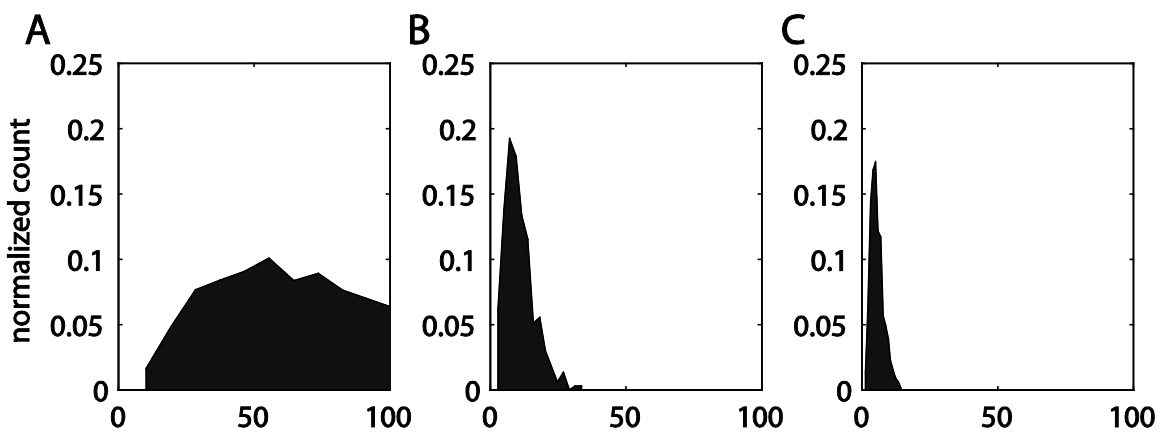

CV\% (connection strength)

CV\% (node degree)

CV\% (node cluster coefficient)

Figure 2.6 Histogram of coefficients of variation. Histogram of coefficients of variation for (A) connection strength of pairs of brain regions, (B) mean node degree of individual nodes, (C) cluster coefficient for individual nodes. CV for connection strength is more widely distributed and on average higher, indicating that connection strength is not well reproducible.

A

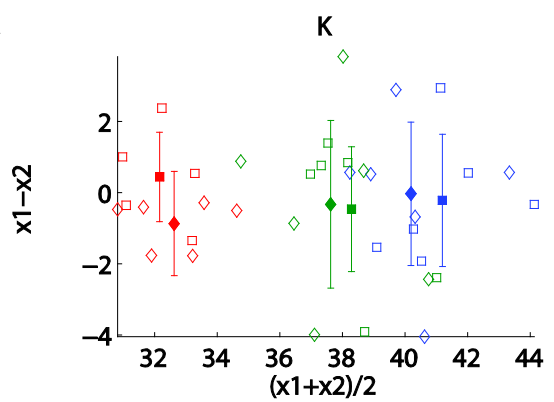

C

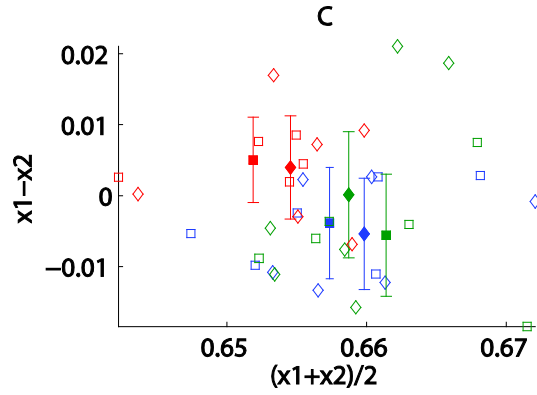

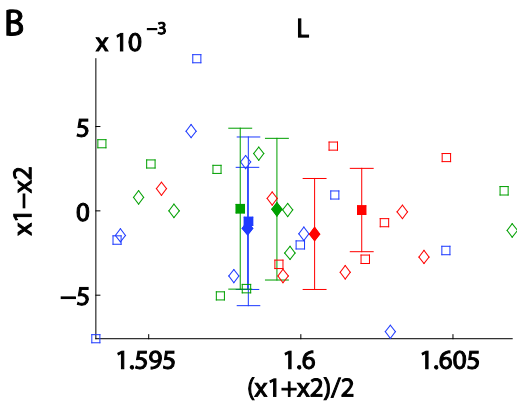

D

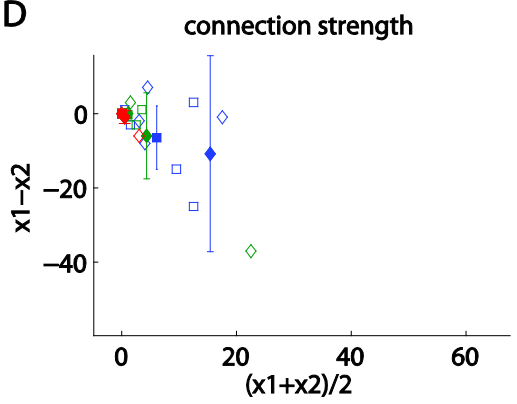

Figure 2.7 Bland-Altman plots for small-world metrics and tract connection strength. Bland-Altman plots showing the difference in measured quantities from the two sessions ( $y$-axis) as function of the average measured quantities (x-axis) for (A) node degree, (B) characteristic path length (at sparsity $=0.74),(C)$ cluster coefficient (at sparsity $=0.74)$, and $(D)$ connection strength. The values for connection strength in (D) are from the fiber connections between BA 6 left to BA 19 right. The mean of the group measurement differences ( $y$-axes) and one standard deviation (errorbar) against the mean of the group measurement means (x-axes) is also represented (filled markers and error bars). The bias introduced by choice of gradient scheme can be appreciated. 


\section{DISCUSSION}

This is, to our knowledge, the first report on the assessment of the reproducibility of structural brain network characteristics derived from tractography data. The observed interscan reproducibility of the network measures used in this study was high, advocating the use of graph theoretical measurements to study neurological diseases. The influence of DTI gradient scheme on tractography and the corresponding computational measures from structural brain networks was investigated. Small-world metrics were dependent on the directional resolution, but not the amplitude, of the gradient scheme. The interscan reproducibility did not depend on choice of the gradient scheme.

\section{Effect of DTI protocol on small world metrics}

\section{Non-thresholded networks}

Mean node degree for the non-thresholded networks varied with the applied gradient scheme. Utilization of fewer gradient directions was associated with shorter tracts. This also had an effect on small world metrics: long range connections were found less often in the gradient schemes with fewer directions. The reduced number of connections due to a lower number of gradient directions was apparent through the decreased $K$. A decrease in $K$ implies that, on average, nodes appear to be connected to fewer nodes.

\section{Thresholded networks}

For the thresholded networks, an increase in average path length between brain areas was notable through an increased $L$ value. The cluster coefficient for these networks also showed a decrease, implying that fewer clusters were found in the network. The effect of number of gradient directions on $C$ was less significant than on $L$ values (Table 2.3). Most clusters are local, thus are formed by anatomically close brain areas, these are influenced less by the absence of long range connections. This is suggested by the skewed distribution of tract lengths shown in Figure 2.4, where an abundance of short tracts is visible.

The metrics discussed above were the absolute values of $L$ and $C$. When investigating whether a network has small-world properties, the values of $L$ and $C$ should be compared to values generated from random networks. The values of $\lambda=L_{\text {real }} / L_{\text {rand }}$ and $\gamma=C_{\text {real }} / C_{\text {rand }}$ show significant difference with directional resolution $(p<0.001)$. In addition to the angular resolution it might be expected that the network resolution (i.e. number and size of nodes) of the image analysis and the spatial resolution (i.e. voxel size) of the image acquisition affect the network metrics. Zalesky et al. (Zalesky et al., 2009) and Hagmann et al. (Hagmann et al., 2008) recently showed that the parcellation scale strongly influences the network metrics. However, it is also reported 
that this strong dependence does not suggest that any given parcellation scale is more optimal than another. Regarding the spatial resolution it is a priori not clear whether smaller voxel sizes will influence the network metrics, as smaller voxels exhibit a lower signal-to-noise ratio (leading to a larger cone of uncertainty) but a more homogeneous within-voxel fiber distribution (leading to a smaller cone of uncertainty) (Parker et al., 2003). Preliminary investigations reveal that these mechanisms appear to compensate to a large extent and therefore do not strongly affect the network metrics (see supplementary Figure S.2.3).

\section{Reproducibility of small world metrics}

The network metrics all produced very low coefficients of variation $(<3.8 \%)$, advocating the applicability of these measures in clinical studies to detect small effects. Even though differences in absolute values of the small world metrics were evident, there was no significant difference in CV between different gradient sampling schemes. All sampling schemes were matched for total scan time, resulting in an increased number of signal averages for the schemes with lower directional resolution. Most likely, the averaging of an increased number of images compensated for the increased directional bias resulting from a low number of gradient directions.

ICC values varied greatly and were not as high as one might desire at first sight. Taking into consideration that the study population was very homogeneous (healthy young adults, with comparable age, educational level and demographics), the between subject variation in this sample is expectedly much lower than for the general (healthy) population or a specific patient population, which gives rise to low ICC. Furthermore, the ICC values found in our study are comparable to the ICC values reported in (Deuker et al., 2009), in which the reproducibility of graph metrics from whole brain MEG functional networks were investigated in healthy volunteers.

\section{Comparison of small world and tract metrics}

Compared to quantitative measures of number of tracts, the small world metrics show much lower CVs (Figure 2.6). This indicates that network measurements from the brain graph are more reliable than connection strengths between pairs of brain regions.

Local tract based quantifications are in practice hard to accomplish as precise, possibly observer dependent, VOI placement and multiple VOI approaches are required (Wakana et al., 2007). Global analysis approaches such as the one presented in this paper, are more robust against errors induced by preprocessing steps such as coregistration and normalization as shown in Figure 2.1. The network metrics are calculated from measurement data from the whole brain comprising many tracts and thus are less affected by noise compared to single tracts which only result from a small amount of the total data. This is reflected in the lower CV values for small-world metrics compared to single tract connection strength (Figure 2.6). 


\section{Limitations}

As illustrated in Figure 2.1, various factors of the data processing steps influence the determined small-world metrics. The results are dependent on the quality and accuracy of the VOI placement. The labeling of grey-white matter voxels to a certain Brodmann area is subject to inaccuracies in the inverse normalization step. Also the definition of the grey-white matter interface depends on the quality of the tissue segmentation and co-registration step. Furthermore, it is not clear whether the definition of the Brodmann areas is indeed a good representation of a structural node in the brain network. However, the subject of accurate brain parcellation based on tractography is still an active area of research and at present remains unfeasible for whole brain data (Johansen-Berg et al., 2004).

The highest number of gradient directions in the present study is 32 .This number is generally thought to be insufficient to accurately model multiple fiber directions per voxel (Alexander et al., 2002; Behrens et al., 2007) and for use in more advanced diffusion reconstruction models, such as Q-ball (Tuch, 2004) or DOT (Ozarslan et al., 2006). Such non-tensor methods might have great consequences for tractography and the influence on small-world metrics needs to be explored. In the current study, the differences in small world metrics appear to decrease with increasing number of diffusion directions (Figure 2.5). Therefore, further increasing the number of diffusion directions is expected to yield even smaller differences that converge to asymptotic values. With the current data it is not possible to investigate this. However, the gradient schemes investigated are clinically available, have relatively short scan times and appear to provide reproducible network parameters. This greatly increases the applicability of the current results for clinical studies, were constraints on patient scanning time are an important issue.

In this study only six healthy subjects were imaged twice. For more accurate estimations of the reproducibility measures more subjects and more than two repeated measurements would be required. It would also be important to investigate whether reproducibility values are similar in relevant patient groups, where intersubject variations are likely to be much higher.

\section{Clinical applicability}

The repeatability coefficient $(\mathrm{RC})$ gives an indication of smallest detectable differences that are biologically relevant. As such, it can be compared to known values from literature reporting on differences between healthy and diseased subjects. In Table 2.5 small world metrics from literature (Liu et al., 2008; Supekar et al., 2008; Shu et al., 2009) are shown and compared to RC values calculated in the present study. RC values are in all cases comparable and in most cases smaller that the reported differences. These findings support the notion presented in this paper that small world metrics derived from whole brain tractography data has potential as a clinical disease marker. 
There have been several studies reporting on network measures from whole brain data and even though these involve different imaging modalities such as $\mathrm{fMRI}$ (Liu et al., 2008; Supekar et al., 2008) and EEG (Stam et al., 2007), which measure different physiological properties of the brain, the current results can be compared with these studies. The agreement between small world metrics derived from structural (tractography) and functional (fMRI and EEG correlation) networks indicates a strong correspondence between the default mode functional network and the underlying structural network (Park et al., 2008; Skudlarski et al., 2008). In a recent paper by Li et al. (Li et al., 2009), a relation between brain structural network properties and intelligence was shown. Cognitive impairment or decline is often an important marker in neurological diseases and if these markers are directly related to quantitative networks metrics from tractography data, the assessment of small world brain connectivity might play an important role in the development of a more mechanistic understanding of the relation between cognitive abilities and micro-structural properties of the brain and how it is affected by disease.

In the work by Itturia (Iturria-Medina et al., 2008) and Gong (Gong et al., 2008), DTI measurements were also used to determine small-world metrics. There are considerable differences in methodology between these studies and ours, including gradient sets, brain atlas and tractography method. However, the results reported in this study agree with those studies to some extent. The differences might in part be explained by the number of gradient directions applied, as the values for $L, C$ and $\lambda$ in those studies show the same effect for number of gradient directions as found in our study ( 6 and 12 for Itturia et al. and Gong et al. respectively). For instance, $\lambda$ increases with number of gradient directions. A summary of the reported values in the above mentioned studies can be found in Table 2.6.

All applied DTI protocols give rise to a high interscan reproducibility and are therefore suitable for studies comparing patient groups with controls or longitudinal studies. This overall high reproducibility does not mean that there is no preference for any of the protocols. The small world metrics were dependent on the number of gradient directions applied. As the average node degree reveals stronger connectivity for gradient schemes with increasing number of directions, the largest scheme is expected to be the most accurate.

\section{CONCLUSIONS}

The choice of gradient acquisition scheme had a significant effect on tractography of DTI data and the resulting small world metrics. More gradient directions resulted in longer tracts and more densely connected graphs. Even though choice of gradient scheme did not influence the reproducibility of the measurements, the relative absence of long range tracts in the acquisition schemes with low directional resolution makes these schemes less favorable. The high reproducibility of the graph theoretical 
measurements of structural connectivity found in this study advocates their clinical applicability. As the number of gradient directions applied potentially introduces a bias in the network results, the use of any gradient scheme should be carefully considered when comparing results across studies or designing new studies.

Table 2.5 Comparison of Repeatability Coefficient with published differences of small-world metrics. The repeatability coefficient $(\mathrm{RC})$ for characteristic path length $(L)$, Cluster coefficient $(C), \lambda=L /$ $L_{\text {random }}, \gamma=C / C_{\text {random' }}$ (see text) and $\sigma=\gamma / \lambda$, for the '32' scheme at sparsity $=0.74$, is compared with reported differences from control and disease groups. An ' $*$ ' indicated the values were significantly different between patient and control group.

\begin{tabular}{llllll}
\hline & $L$ & $C$ & $\gamma$ & $\sigma$ & Imaging modality \\
\hline Present study & 0.007 & 0.010 & 0.090 & 0.100 & $\begin{array}{l}\text { DTI / Probabilistic } \\
\text { Tractography }\end{array}$ \\
$\begin{array}{l}\text { Supekar } \\
\text { (control - patient) }\end{array}$ & - & - & $0.18^{*}$ & $0.16^{*}$ & $\begin{array}{l}\text { RS-fMRI } \\
\text { Liu } \\
\text { (control - patient) }\end{array}$ \\
$\begin{array}{l}\text { Shu } \\
\text { (control - patient) }\end{array}$ & $-0.051^{*}$ & $0.023 / 0.013^{*}$ & 0.068 & $0.06^{*}$ & RS-fMRI \\
\hline $\begin{array}{l}1 \\
\text { fMRI Resting State (Supekar et al., 2008). }\end{array}$ & $-0.069^{*}$ & -0.0013 & -0.01 & -0.09 & DTI / Deterministic \\
fMRI Resting State (Liu et al., 2008). & & & & & Tractography \\
${ }^{3}$ Tractography (Shu et al., 2009). & & & & &
\end{tabular}

Table 2.6 Comparison of small-world metric from selected literature and our study. Small-world metrics (characteristic path length $(L)$, Cluster coefficient $(C), \lambda=L / L_{\text {random }}, \gamma=C / C_{\text {random }}$ and $\sigma=\lambda / \gamma)$ from the ' 32 ' scheme at sparsity=0.74 compared with published values from literature.

\begin{tabular}{|c|c|c|c|c|c|c|}
\hline & $L$ & $C$ & $\lambda$ & Y & $\sigma$ & Imaging modality \\
\hline Present study & 1.62 & 0.65 & 1.02 & 2.28 & 2.24 & DTI / Probabilistic Tractography \\
\hline Ituria ${ }^{1}$ & - & - & 1.12 & 1.85 & 1.64 & DTI / Deterministic Tractography \\
\hline Gong $^{2}$ & 2.32 & 0.49 & 1.15 & 4.07 & - & DTI / Deterministic Tractography \\
\hline $\mathrm{Li}^{3}$ & 2.81 & 0.49 & 1.14 & 2.07 & - & DTI / Deterministic Tractography \\
\hline $\mathrm{Shu}^{4}$ & 2.17 & 0.50 & 1.08 & 1.73 & 1.60 & DTI / Deterministic Tractography \\
\hline Hagmann ${ }^{5}$ & - & - & - & - & 1.54 & DTI / Deterministic Tractography \\
\hline $\operatorname{Liu}^{6}$ & - & - & 1.02 & 1.57 & - & RS-fMRI \\
\hline Superkar ${ }^{7}$ & - & - & 1.05 & 1.74 & - & RS-fMRI \\
\hline Stam $^{8}$ & - & - & 1.07 & 1.58 & - & EEG \\
\hline Archard $^{9}$ & 2.49 & 0.525 & 1.08 & 2.38 & 2.19 & RS-fMRI \\
\hline
\end{tabular}

${ }^{1}$ Tractography (Iturria-Medina et al., 2008).

${ }^{2}$ Tractography (Gong et al., 2008).

${ }^{3}$ Tractography (Li et al., 2009).

${ }^{4}$ Tractography (Shu et al., 2009).

${ }^{5}$ Diffusion sprectrum imaging (DSI) tractography (Hagmann et al., 2008).

${ }^{6} \mathrm{fMRI}$ resting state (Liu et al., 2008).

${ }^{7} \mathrm{fMRI}$ resting state (Supekar et al., 2008).

${ }^{8}$ EEG (Stam et al., 2007).

${ }^{9}$ fMRI (Achard and Bullmore, 2007). 


\section{REFERENCES}

Achard S, Bullmore E (2007) Efficiency and Cost of Economical Brain Functional Networks. PLoS Comput Biol 3:e17.

Alexander DC, Barker GJ, Arridge SR (2002) Detection and modeling of non-Gaussian apparent diffusion coefficient profiles in human brain data. Magn Reson Med 48:331-340.

Ashburner J, Friston KJ (1999) Nonlinear spatial normalization using basis functions. Hum Brain Mapp 7:254266.

Bassett DS, Bullmore E (2006) Small-world brain networks. Neuroscientist 12:512-523.

Behrens TE, Berg HJ, Jbabdi S, Rushworth MF, Woolrich MW (2007) Probabilistic diffusion tractography with multiple fibre orientations: What can we gain? Neuroimage 34:144-155.

Behrens TE, Woolrich MW, Jenkinson M, Johansen-Berg H, Nunes RG, Clare S, Matthews PM, Brady JM, Smith SM (2003) Characterization and propagation of uncertainty in diffusion-weighted MR imaging. Magn Reson Med 50:1077-1088.

Bland JM, Altman DG (1986) Statistical methods for assessing agreement between two methods of clinical measurement. Lancet 1:307-310.

Bridge $\mathrm{H}$, Thomas O, Jbabdi S, Cowey A (2008) Changes in connectivity after visual cortical brain damage underlie al tered visual function. Brain 131:1433-1444.

Cook PA, Bai Y, Nedjati-Gilani S, Seunarine KK, Hall MG, Parker GJ, Alexander DC (2006) Camino: OpenSource Diffusion-MRI Reconstruction and Processing. In: 14th Scientific Meeting of the International Society for Magnetic Resonance in Medicine.

Deuker L, Bullmore ET, Smith M, Christensen S, Nathan PJ, Rockstroh B, Bassett DS (2009) Reproducibility of graph metrics of human brain functional networks. Neuroimage 47:1460-1468.

Farrell JA, Landman BA, Jones CK, Smith SA, Prince JL, van ZijI PC, Mori S (2007) Effects of signal-to-noise ratio on the accuracy and reproducibility of diffusion tensor imaging-derived fractional anisotropy, mean diffusivity, and principal eigenvector measurements at $1.5 \mathrm{~T}$. J Magn Reson Imaging 26:756-767.

Gong G, He Y, Concha L, Lebel C, Gross DW, Evans AC, Beaulieu C (2008) Mapping Anatomical Connectivity Patterns of Human Cerebral Cortex Using In Vivo Diffusion Tensor Imaging Tractography. Cereb Cortex:bhn102.

Greicius MD, Supekar K, Menon V, Dougherty RF (2009) Resting-state functional connectivity reflects structural connectivity in the default mode network. Cereb Cortex 19:72-78.

Hagmann P, Cammoun L, Gigandet X, Meuli R, Honey CJ, Wedeen VJ, Sporns O (2008) Mapping the structural core of human cerebral cortex. PLoS Biol 6:e159.

Honey CJ, Kotter R, Breakspear M, Sporns O (2007) Network structure of cerebral cortex shapes functional connectivity on multiple time scales. Proc Natl Acad Sci U S A 104:10240-10245.

Humphries MD, Gurney K, Prescott TJ (2006) The brainstem reticular formation is a small-world, not scalefree, network. Proc Biol Sci 273:503-511.

Iturria-Medina Y, Sotero RC, Canales-Rodriguez EJ, Aleman-Gomez Y, Melie-Garcia L (2008) Studying the human brain anatomical network via diffusion-weighted MRI and Graph Theory. Neuroimage 40:1064-1076.

Johansen-Berg H, Behrens TE, Robson MD, Drobnjak I, Rushworth MF, Brady JM, Smith SM, Higham DJ, Matthews PM (2004) Changes in connectivity profiles define functionally distinct regions in human medial frontal cortex. Proc Natl Acad Sci U S A 101:13335-13340.

Lachin JM (2004) The role of measurement reliability in clinical trials. Clin Trials 1:553-566. 
Landman BA, Farrell JA, Jones CK, Smith SA, Prince JL, Mori S (2007) Effects of diffusion weighting schemes on the reproducibility of DTI-derived fractional anisotropy, mean diffusivity, and principal eigenvector measurements at 1.5T. Neuroimage 36:1123-1138.

Le Bihan D, Mangin JF, Poupon C, Clark CA, Pappata S, Molko N, Chabriat H (2001) Diffusion tensor imaging: concepts and applications. J Magn Reson Imaging 13:534-546.

Li Y, Liu Y, Li J, Qin W, Li K, Yu C, Jiang T (2009) Brain anatomical network and intelligence. PLoS Comput Biol 5:e1000395.

Liu Y, Liang M, Zhou Y, He Y, Hao Y, Song M, Yu C, Liu H, Liu Z, Jiang T (2008) Disrupted small-world networks in schizophrenia. Brain 131:945-961.

Maldjian JA, Laurienti PJ, Kraft RA, Burdette JH (2003) An automated method for neuroanatomic and cytoarchitectonic atlas-based interrogation of fMRI data sets. Neuroimage 19:1233-1239.

Maslov S, Sneppen K (2002) Specificity and stability in topology of protein networks. Science 296:910-913.

Ozarslan E, Shepherd TM, Vemuri BC, Blackband SJ, Mareci TH (2006) Resolution of complex tissue microarchitecture using the diffusion orientation transform (DOT). Neuroimage 31:1086-1103.

Park C-h, Kim SY, Kim Y-H, Kim K (2008) Comparison of the small-world topology between anatomical and functional connectivity in the human brain. Physica A: Statistical Mechanics and its Applications 387:5958-5962.

Parker GJ, Haroon HA, Wheeler-Kingshott CA (2003) A framework for a streamline-based probabilistic index of connectivity (PICo) using a structural interpretation of MRI diffusion measurements. Journal of Magnetic Resonance Imaging 18:242-254.

Powell HW, Parker GJ, Alexander DC, Symms MR, Boulby PA, Wheeler-Kingshott CA, Barker GJ, Koepp MJ, Duncan JS (2007) Abnormalities of language networks in temporal lobe epilepsy. Neuroimage 36:209-221.

Rilling JK, Glasser MF, Preuss TM, Ma X, Zhao T, Hu X, Behrens TE (2008) The evolution of the arcuate fasciculus revealed with comparative DTI. Nat Neurosci 11:426-428.

Shu N, Liu Y, Li J, Li Y, Yu C, Jiang T (2009) Altered anatomical network in early blindness revealed by diffusion tensor tractography. PLoS One 4:e7228.

Skare S, Hedehus M, Moseley ME, Li TQ (2000) Condition number as a measure of noise performance of diffusion tensor data acquisition schemes with MRI. J Magn Reson 147:340-352.

Skudlarski P, Jagannathan K, Calhoun VD, Hampson M, Skudlarska BA, Pearlson G (2008) Measuring brain connectivity: diffusion tensor imaging validates resting state temporal correlations. Neuroimage 43:554-561.

Smith SM (2002) Fast robust automated brain extraction. Human Brain Mapping 17:143-155.

Smith SM, Jenkinson M, Wool rich MW, Beckmann CF, Behrens TE, Johansen-Berg H, Bannister PR, De Luca M, Drobnjak I, Flitney DE, Niazy RK, Saunders J, Vickers J, Zhang Y, De Stefano N, Brady JM, Matthews PM (2004) Advances in functional and structural MR image analysis and implementation as FSL. Neuroimage 23 Suppl 1:S208-219.

Sporns O, Zwi JD (2004) The small world of the cerebral cortex. Neuroinformatics 2:145-162.

Stam CJ, Reijneveld JC (2007) Graph theoretical analysis of complex networks in the brain. Nonlinear Biomed Phys 1:3.

Stam CJ, Jones BF, Nolte G, Breakspear M, Scheltens P (2007) Small-world networks and functional connectivity in Alzheimer's disease. Cereb Cortex 17:92-99.

Strogatz SH (2001) Exploring complex networks. Nature 410:268-276.

Sundgren PC, Dong Q, Gomez-Hassan D, Mukherji SK, Maly P, Welsh R (2004) Diffusion tensor imaging of the brain: review of clinical applications. Neuroradiology 46:339-350.

Supekar K, Menon V, Rubin D, Musen M, Greicius MD (2008) Network analysis of intrinsic functional brain 
connectivity in Alzheimer's disease. PLoS Computational Biology 4:e1000100.

Tijssen RH, Jansen JF, Backes WH (2008) Assessing and minimizing the effects of noise and motion in clinical DTI at 3 T. Hum Brain Mapp.

Toosy AT, Ciccarelli O, Parker GJ, Wheeler-Kingshott CA, Miller DH, Thompson AJ (2004) Characterizing function-structure relationships in the human visual system with functional MRI and diffusion tensor imaging. Neuroimage 21:1452-1463.

Tuch DS (2004) Q-ball imaging. Magn Reson Med 52:1358-1372.

Wakana S, Caprihan A, Panzenboeck MM, Fallon JH, Perry M, Gollub RL, Hua K, Zhang J, Jiang H, Dubey P, Blitz A, van Zijl P, Mori S (2007) Reproducibility of quantitative tractography methods applied to cerebral white matter. Neuroimage 36:630-644.

Watts DJ, Strogatz SH (1998) Collective dynamics of 'small-world' networks.[see comment]. Nature 393:440442.

Yogarajah M, Duncan JS (2008) Diffusion-based magnetic resonance imaging and tractography in epilepsy. Epilepsia 49:189-200.

Zalesky A, Fornito A, Harding IH, Cocchi L, Yucel M, Pantelis C, Bullmore ET (2009) Whole-brain anatomical networks: Does the choice of nodes matter? Neuroimage. 


\section{SUPPLEMENTARY DATA}

A

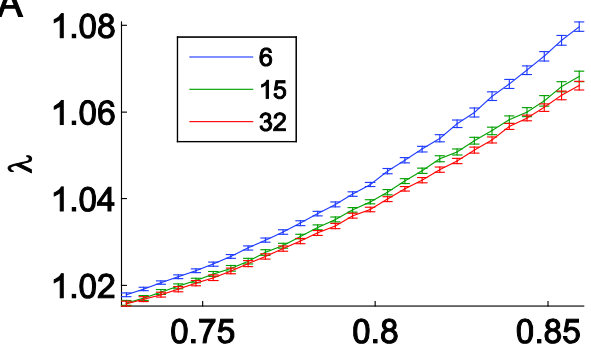

B

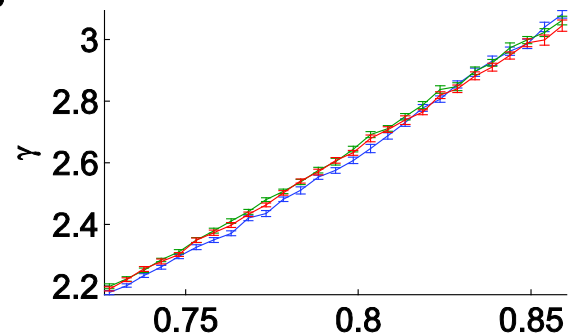

C

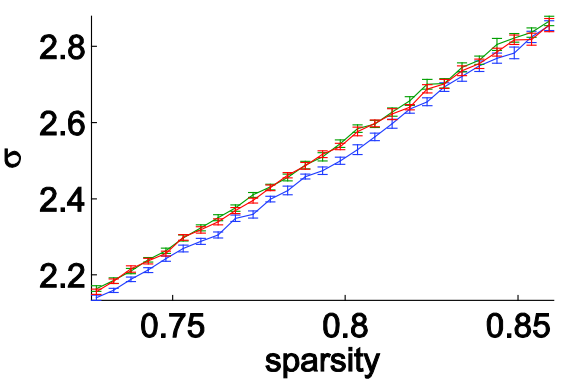

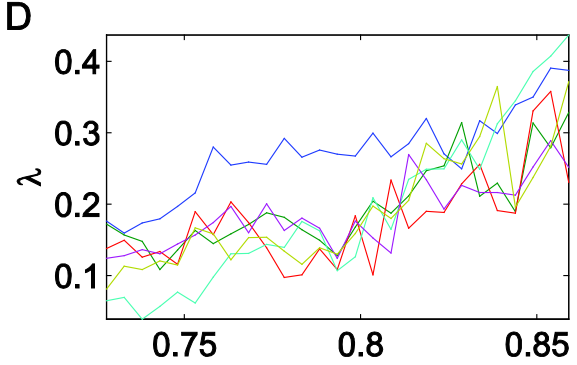

E

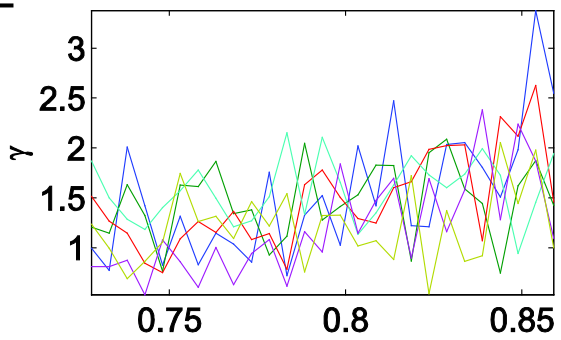

$\mathbf{F}$

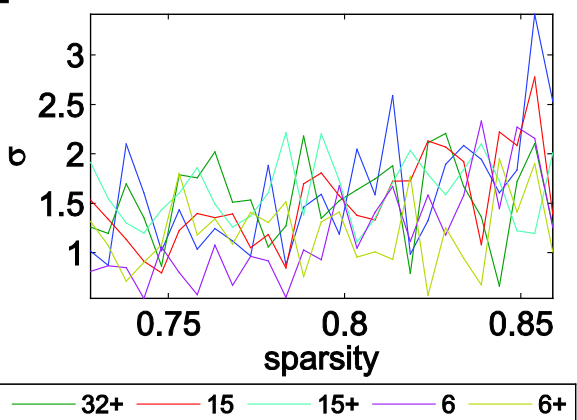

Figure S.2.1 Values and CV for $\lambda, \gamma$ and $\sigma$ as a function of sparsity. Sub panels A, B and C show the values for $\lambda, \gamma$ and $\sigma$, respectively. Al three metrics increase with sparsity, indicating a shift away from the topology of a random network. Subpanels $\mathbf{D}, \mathbf{E}$ and $\mathbf{F}$ show the corresponding CV values. The CV for all three metrics is low and only slightly increases with sparsity. 

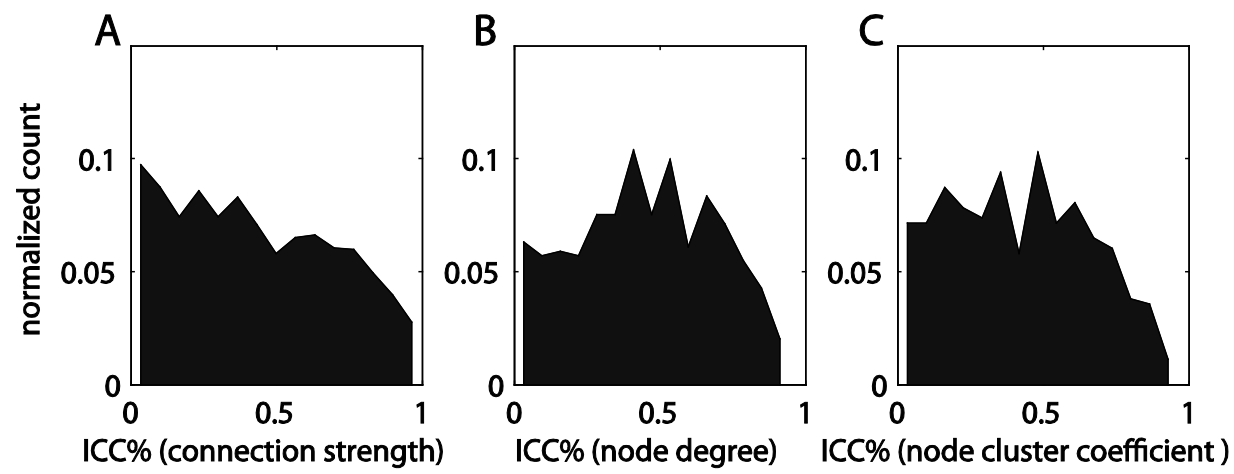

Figure S.2.2 Histogram of ICC values for (A) connection strength of pairs of brain regions, (B) node degree of individual nodes and (C) cluster coefficient for individual nodes.

A

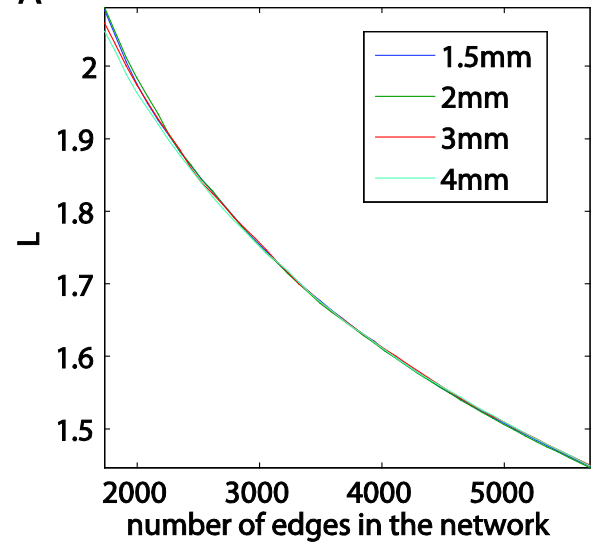

B

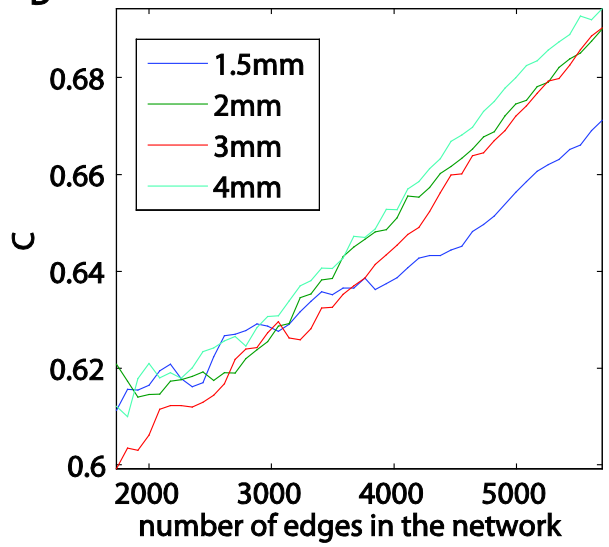

Figure S.2.3 Small world metrics $L(\mathbf{A})$ and $C(\mathbf{B})$ as a function of number of edges for different acquired voxel sizes obtained in a healthy subject. Note that there is no strong effect of the voxel size on $L$ or $C$. 
56 Chapter 2 


\section{CHAPTER 3}

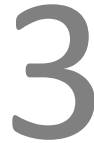

\section{Loss of functional network efficiency associated with cognitive decline in chronic epilepsy}

M.J. Vaessen*, M.C.G. Vlooswijk*, J.F.A. Jansen, M.C.F.T.M. de Krom, H.J.M. Majoie,

P.A.M. Hofman, A.P. Aldenkamp, W.H. Backes

*both authors contributed equally 


\section{ABSTRACT}

To study the relation between possibly altered whole brain topology and intellectual decline in chronic epilepsy, a combined study of neurocognitive assessment and graph theoretical network analysis of fMRI was performed.

Forty-one adult patients with cryptogenic localization-related epilepsy and 23 healthy controls underwent an intelligence test and $\mathrm{FMRI}$ with a silent-word generation paradigm. A set of undirected graphs was constructed by cross-correlating the signal time series of 893 cortical and subcortical regions. Possible changes in cerebral network efficiency were assessed by performing graph theoretical network analysis.

Healthy subjects displayed efficient small world properties, characterized by high clustering and short path lengths. On the contrary, in patients with epilepsy a disruption of both local segregation and global integration was found. An association of more pronounced intellectual decline with more disturbed local segregation was observed in the patient group. The effect of antiepileptic drug use on cognitive decline was mediated by decreased clustering.

These findings support the hypothesis that chronic localization-related epilepsy causes cognitive deficits by inducing global cerebral network changes instead of a localized disruption only. Whether this is the result of epilepsy per se or the use of antiepileptic drugs remains to be elucidated. For application in clinical practice, future studies should address the relevance of altered cerebral network topology in prediction of cognitive deficits and monitoring of therapeutic interventions. 


\section{INTRODUCTION}

In chronic epilepsy, patients often experience cognitive problems (Fisher et al., 2000) extending from memory deficits (Helmstaedter and Kurthen, 2001) to language problems (Vlooswijk et al., 2010a) and intellectual impairment (Oyegbile et al., 2004). Clinical factors such as antiepileptic drugs (AED) (Jokeit et al., 2005), and high seizure frequency (Thompson and Duncan, 2005) cannot always predict the individual cognitive course.

To understand the neurobiological mechanisms of cognitive dysfunction in localization-related epilepsy, functional MRI (fMRI) research has focused on changes in activation patterns. Most fMRI studies report an association of cognitive dysfunction with either decreased activation (Meletti et al., 2003; Cheung et al., 2006; Powell et al., 2007) or a shift of activation (Dupont et al., 2001; Weber et al., 2006; Vlooswijk et al., 2010b).

The focus of $\mathrm{fMRI}$ research in epilepsy and cognition has changed to analyzing dysfunctional networks. Most studies measure functional connectivity by correlating signal time-courses of different cerebral regions (Waites et al., 2006). Typically, higher functional connectivity is associated with better cognitive performance (Addis et al., 2007; Bettus et al., 2009; Zhang et al., 2009).

With conventional functional connectivity methods applying a priori selection of specific networks unexpected abnormalities outside these networks can remain undetected. With graph theoretical analysis the organization of the whole brain network can be investigated (for a review, see (Stam and Reijneveld, 2007)). A distinction can be made between a 'small-world' and a random topology. Most studies (Bullmore and Sporns, 2009) demonstrate that brain networks are organized as 'small-world' networks which are more efficient than 'random' networks (van den Heuvel et al., 2009).

We aimed to investigate the changes in functional networks using graph theoretical network analysis in patients with epilepsy in relation to intellectual performance and possible decline. We hypothesize that: (i.) patients will have lower intellectual performance; (ii.) patients will have a less efficient organized network than healthy controls; and (iii.) network abnormalities will be more pronounced in those patients with lowest IQ and distinct intellectual decline.

\section{METHODS}

\section{Participants}

Inclusion criteria for the patients were: localization-related epilepsy with an epileptic focus in the frontal or temporal lobe, absence of structural cerebral lesions other than mesiotemporal sclerosis (MTS), no history of status epilepticus and no other disease 
that could cause cognitive decline. The final study population included 41 patients (21 women; mean age 40 years; range 22-63) and 23 healthy controls (14 women; mean age 40 years; range 18-58). See Supplemental Table S.3.1 for an overview of the clinical characteristics of the patients and healthy controls.

\section{Standard protocol approvals, registrations, and patient consents}

This study was approved by the Institutional Review Board of the Maastricht University Medical Center. All subjects gave written informed consent.

\section{Neuropsychological testing}

For intelligence, the third Wechsler Adult Intelligence Scale (WAIS-III) was used (Wechsler, 1997). An estimate of premorbid intelligence levels was made according to the formula proposed by Schoenberg et al (Schoenberg et al., 2002). Intelligence discrepancy scores were calculated by subtracting premorbid full-scale IQ (FSIQ) estimates from actual FSIQ, resulting in IQ discrepancy scores (see Supplemental data for details).

\section{MRI protocol}

All subjects underwent a clinical epilepsy protocol on a 3.0-T unit with an 8-channel head coil. Functional MRI data were acquired using echo-planar imaging pulse sequence $\left(\mathrm{TR}=2 \mathrm{~s}, \mathrm{TE}=5 \mathrm{~ms}, \mathrm{FA}=90^{\circ}\right.$, voxel size $2 \times 2 \times 4 \mathrm{~mm}$ ) and 196 volumes per acquisition. For anatomic reference, a T1-weigthed 3D fast gradient echo was acquired $\left(\mathrm{TR}=9.91 \mathrm{~ms}, \mathrm{TE}=4.6 \mathrm{~ms}, \mathrm{Tl}=3 \mathrm{~s}, \mathrm{FA}=8^{\circ}\right.$, voxel size $\left.1 \times 1 \times 1 \mathrm{~mm}\right)$.

\section{fMRI activation paradigm}

In the word generation paradigm, subjects had to covertly generate as many words as possible starting with a visually presented letter (U-N-K-A-E-P). The paradigm consisted of six word-generation condition blocks (one letter per $30 \mathrm{~s}$ block) alternated with baseline rest condition blocks (30 s). Afterwards, all subjects were able to sufficiently reproduce words generated during the task.

\section{Image analysis}

\section{Image preprocessing}

Analysis of the time-series data was performed in the Statistical Parametric Mapping (SPM2) software application (Wellcome Department of Cognitive Neurology, UK). Dynamic images were slice-timed and realigned to correct for head movement. The corrected images were transformed into standard MNI space and spatially smoothed (6-mm kernel). 


\section{Whole brain network construction}

The preprocessed and normalized $\mathrm{fMRI}$ images were parcellated into a high resolution network consisting of $\mathrm{N}=893$ cortical and subcortical brain regions (see Supplemental data for details on the parcellation scheme). Characteristic time-series were calculated by averaging the signal intensities from all voxels in a region. To reduce the effect physiological noise and movement related noise (Van Dijk et al., 2010), the time-series were filtered by applying standard linear regression with the movement parameters as a co-variate and by applying a bandpass filter $(0.01-0.1 \mathrm{~Hz})$.

\section{Network parameters}

Graph theoretical parameters were used to evaluate the functional networks (Watts and Strogatz, 1998; Bassett and Bullmore, 2006; Stam and Reijneveld, 2007). A connection matrix $A$ was formed by calculating Pearson correlation coefficients between all pairs of brain regions. In the brain graph, a node is related to a brain region, an edge is a connection between brain areas $i$ and $j$.

The brain graph of each individual was thresholded to create graphs with an equal number of nodes and edges across subjects (Stam et al., 2007). This was achieved by selecting the $T_{k}$ connections with the highest correlation coefficient and removing all other connections. The threshold value $T_{k}$ was expressed as a sparsity value relating the number of edges maintained in the network to the total number of edges possible $\left(N^{2}-N\right)$ (Achard and Bullmore, 2007). Let $T_{k}$ be the number of edges maintained in the network, then the sparsity (s) is defined as:

$$
\text { sparsity }=\left(N^{2}-N-T_{k}\right) /\left(N^{2}-N\right)
$$

As there is no theoretical criterion for which sparsity value is the most biologically meaningful, here we explored network parameters over a range of sparsity values. To guarantee high correlation coefficients of the remaining connections, the sparsity range was chosen to be higher than 0.87 , which yielded an average correlation coefficient of 0.66 .

The graph theoretical metrics characteristic path length $(L)$ and cluster coefficient $(C)$ as well as local and global efficiency were calculated to perform analysis on the constructed brain graphs. The characteristic path length is a measure of how well connected a network is. The cluster coefficient of a network is a measure of how many local clusters exist in the network. Parameters related to characteristic path length are global efficiency $\left(E_{\text {global }}\right)$ and local efficiency $\left(E_{\text {local }}\right)$. $E_{\text {global }}$ is defined as the average inverse shortest path length, $E_{\text {local }}$ is defined as the mean of the global efficiencies of subgraphs consisting of the immediate neighbours of a particular node (Achard and Bullmore, 2007). To be able to determine whether a network has smallworld properties, the values of $L$ and $C$ must be scaled to values from generated random networks (Watts and Strogatz, 1998). Small world networks are characterized by having $L$ close to random: $\lambda=L / L_{\text {random }} \approx 1$, but with $C$ higher than random: 
$\gamma=C / C_{\text {random }}>1$. See Supplemental data for a more elaborate description of these metrics.

\section{Statistical analysis}

Group differences of graph theoretical network parameters were assessed with the Student's $t$-test. The associations between clinical variables (age, age at onset and drugload), cognitive variables (FS-IQ and IQ discrepancy) and network parameters were analyzed using Pearson's correlation coefficients. The relation between clinical seizure variables and graph theoretical parameters with intellectual decline was examined using a mediator-model approach (Baron and Kenny, 1986). With this model, it is investigated whether network parameters mediate the relation between clinical and cognitive characteristics. Analyses were performed with graph theoretical network parameters and drug load (the clinical variable with the strongest link to cognition in this dataset) as independent and intellectual decline as the dependent variable. If the relation between drug load and intellectual decline becomes nonsignificant when network parameters are entered in the model, this parameter can be considered a mediator of the relation between drug load and intellectual decline.

\section{RESULTS}

\section{Neuropsychological assessment}

Patients had lower FSIQ than controls $(96 \pm 15$ vs $113 \pm 15, p<0.01)$. In the patient group, IQ discrepancy scores were significantly lower than in the controls, indicating intellectual decline in the patient group as a whole (mean \pm standard deviation IQ discrepancy score $-8.6 \pm 6.5$ in patients vs. $-3.6 \pm 8.8$ in controls, $p=0.02$ ). For the individual patients, seven had lower IQ discrepancy scores than the minimum score in the control group; they had evident intellectual decline (impaired group).

\section{Functional MRI results}

\section{Activation map results}

Activation maps of the word-generation paradigm revealed significantly activated clusters in the left inferior and left middle frontal cortex (Broca region), the right middle frontal cortex, and the anterior cingulate cortex for both groups. No significant differences were found between controls and patients with epilepsy (see (Vlooswijk et al., 2010a) for details). 


\section{fMRI graph theoretical network parameters}

Both patient and control networks showed a topology in the small-world regime with values for $\lambda$ close to 1 and values for $\gamma$ higher than 1 . Patients displayed significantly lower values $(p<0.05)$ for $\gamma, E_{\text {global }}$ and $E_{\text {local }}$ over almost the entire sparsity range (Figure 3.1). A trend towards higher $\lambda$ values was observed in the patient group, which was significantly higher for high sparsity values $(s=0.96-0.97)$. For $C$, patients also had lower values for the highest sparsity values. The network parameters of the impaired group revealed significant lower values for $C$ over a broader sparsity range as compared with unimpaired patients and controls. Impaired patients displayed significantly higher values than controls for $L(s=0.94-0.95)$ and $\lambda(s=0.94-0.96)$ (Supplemental Figure S.3.1).

Summarizing, all the encountered differences point toward a disruption of network integrity characterized by a more random network topology in the patient group.
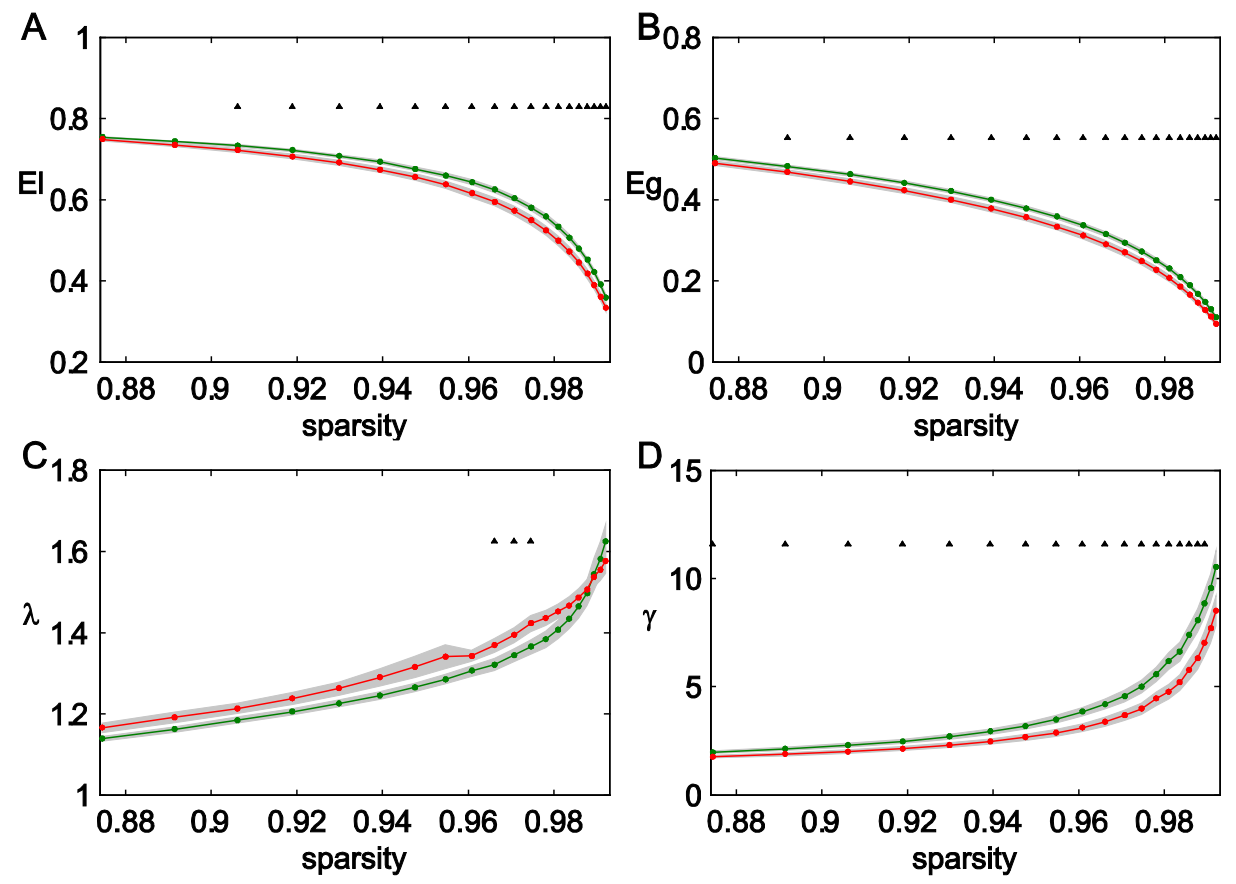

Figure 3.1 Network parameters for the whole brain networks of the patient group (red lines) and the control group (green lines) as function of sparsity. Black triangles indicate for which sparsity values the groups differed significantly, demonstrating consistently lower values for Eg, El and $\gamma$ and higher values for $\lambda$ in the patient group. Grey areas indicate standard error of the mean.

(A) Local efficiency, (B) global efficiency, (C) $\lambda$, and (D) $\gamma$. 

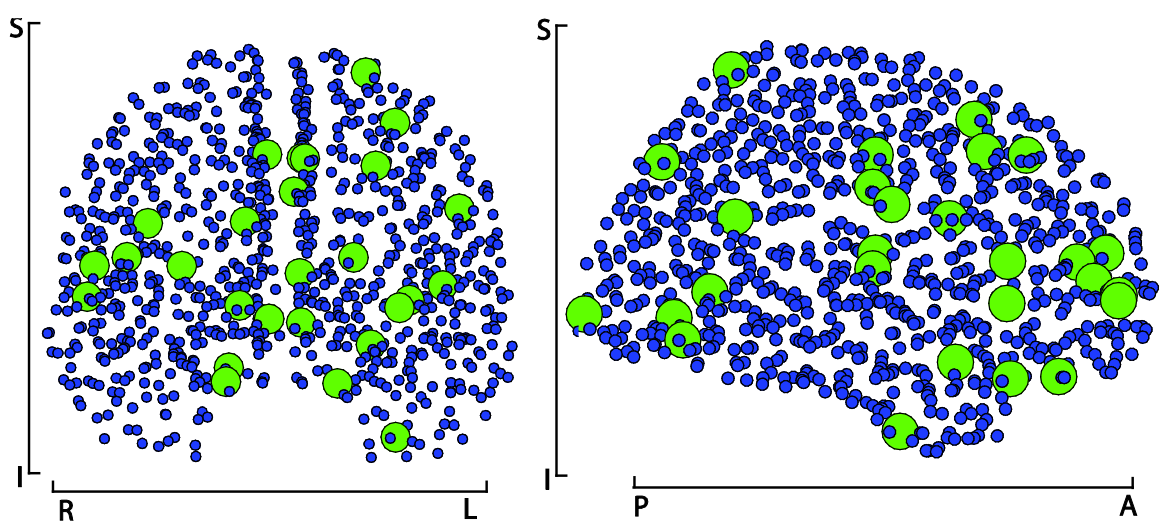

Figure 3.2 3D projection of the network nodes. Green circles indicate where the nodal values of $y$ were significantly lower in the patient group $(p<0.005)$. The affected nodes are distributed throughout the whole brain, not restricted to specific lobes or functional networks. $\mathrm{S}=$ superior; $\mathrm{I}=$ inferior; $\mathrm{L}=$ left; $\mathrm{R}=$ right; $\mathrm{A}=$ anterior; $\mathrm{P}=$ posterior.

\section{Regional analysis of network parameters}

Additional analysis was carried out to investigate whether the majority of identified network abnormalities are localized in certain brain regions or networks. Results are only shown for $\gamma$, since $\gamma$ was the network parameter with the most pronounced difference between patients and controls. As can be observed from Figure 3.2, a number of regions did show significant differences $(p<0.005)$. However, these regions were evenly distributed throughout the whole brain, without evident grouping within a specific lobe or recognizable network. When controls were compared to the impaired patients only, similar results were obtained. No associations of side of seizure focus with distribution of affected regions were observed.

\section{fMRI graph theoretical network parameters in correlation with neuropsychological parameters}

The cluster coefficient was found to be positively associated with FSIQ over a range of sparsity values $(s=0.87-0.97)$. This same effect was seen for the association between $C$ and IQ discrepancy ( $s=0.93-0.98)$. Hence, a decreased amount of clustering in the activated patient brain indicates a reduced $I Q$, and a decreased clustering relates to a more pronounced intellectual decline. For the other network parameters, no correlation with FSIQ and IQ discrepancy was found. In the control group, there was no association between any network parameter and FSIQ. 


\section{Correlation of fMRI graph theoretical network parameters with clinical factors}

From the clinical factors available, drug load was negatively associated with $C, \gamma$ and $E_{\text {local, }}$ indicating a more random and less efficient network with higher drug load (for $C$ : $p<0.03$ for sparsity range $0.97-0.99$; for $\gamma$ : $p$-values range from 0.03 to 0.06 in the entire sparsity range; for $E_{\text {local }}: p<0.05$ for sparsity range $0.87-0.93$ ). For the other clinical characteristics, age and age at onset, no significant associations were found.

\section{Model for the relation between clinical characteristics, network parameters and intellectual decline}

The relation of clinical epilepsy variables and graph theoretical network parameters to intellectual decline was examined using the mediator analysis. Since drug load had the strongest correlation with intellectual decline $(p=0.03)$, this factor was entered in the mediator analysis. The other clinical characteristics did not meet the criteria to be entered in a mediator analysis (Baron and Kenny, 1986). Drug load was no longer a significant predictor of intellectual decline when the clustering coefficient $C$ was included in the model (Supplemental Figure S.3.2). On the contrary, $C$ was the mediating factor between drug load and intellectual decline, indicating that abnormalities in graph theoretical network parameters, particularly the clustering coefficient, mediated the impact of drug load on cognition ( $s=0.96-0.98)$.

\section{DISCUSSION}

In the present study, fMRI time-series data from a language paradigm were used to evaluate functional brain networks in patients with chronic epilepsy and healthy controls. Graph theoretical network parameters were compared between patients and controls and compared with cognitive performance. First, the patients with epilepsy displayed disturbed network parameters, such as a lower normalized clustering coefficient $(\gamma)$, and lower local and global efficiencies. Second, for the subgroup of patients with most pronounced cognitive decline, lower absolute clustering coefficient, and higher absolute and normalized path length were observed. Third, cognitive status (IQ) and the degree of intellectual decline (IQ discrepancy score) were correlated with graph theoretical network parameters, which revealed that poorer cognitive status and more pronounced intellectual decline were associated with lower absolute clustering coefficients. Fourth, as expected (Liu et al., 2008; van den Heuvel et al., 2009), the topological parameters were consistent with a small-world organization of the cerebral networks in the control group.

Lower clustering coefficients (Liu et al., 2008) and an increased path length (Stam et al., 2007; Liu et al., 2008) have been demonstrated in neuropsychiatric disorders such as Alzheimer's disease and schizophrenia. These disorders are accompanied with 
pronounced cognitive deficits, although graph theoretical network parameters have not previously been linked directly with cognitive measures.

Previously, in patients with bilateral mesial temporal lobe epilepsy whole-brain graph network analysis with $\mathrm{fMRI}$ demonstrated lower absolute and normalized path lengths together with a decrease in absolute clustering coefficients (Liao et al., 2010). This was interpreted as a disruption of the whole brain network with a more random topology. The apparent contradictive results concerning path length are difficult to explain due to differences in study population and methodology. For example, patients in the latter study were on average much younger which might have influenced the results. One might hypothesize that brain networks of younger patients respond differently to disease. For instance, young patients might be able to compensate better by acquiring alternate brain regions for cognitive processing, while older patients might have lost this ability. This could lead to a different expression of networks parameters between control and patient groups in different age categories.

Cognitive functioning depends on several cerebral networks instead of isolated brain regions. It is reasonable to assume that in patients with epilepsy a disruption of whole brain networks is involved in the development of cognitive deficits, instead of a localized disruption at the site of seizure focus only. This is supported by MR studies demonstrating volumetric loss (Hermann et al., 2004; Oyegbile et al., 2006), microstructural white matter abnormalities (Focke et al., 2008), cortical thinning (Bernhardt et al., 2010), and functional abnormalities (Bettus et al., 2009; Vlooswijk et al., 2010a) outside the epileptic focus. The application of methods sensitive to overlapping localized abnormalities seems to be limited in patient populations with heterogeneous seizure foci and cognitive deficits over multiple domains. Graph theoretical network analysis allows for analysis of whole brain networks rather than at a local level. Hence, it might be better capable of detecting patient specific abnormalities in functional brain organization and reorganization than more conventional (single connection) analysis methods. Indeed, the disruption of smallworld characteristics in our patient group could not be localized within one or more specialized brain regions, which could represent diffuse disruptions throughout the whole brain. No global technical differences between patients and controls, such as the motion correction parameters, could be identified in this study. The possibility that differences in the location of epileptogenic zone or the use of antiepileptic drugs contribute to the non-localized abnormalities cannot be ruled out. Furthermore, the silent word generation task does not allow for objective assessment of task performance. Theoretically, differences in task performance could affect the network parameters. Nevertheless, based on post-task performance assessment and careful inspection of individual activation maps it is unlikely that salient differences in task performance have influenced the fMRI data.

In normal brain networks, high clustering coefficients ( $C$ and $\gamma$ ) and local efficiency $\left(E_{\text {local }}\right)$ are parameters which reflect a high local specialization (segregation) of information processing. Contrarily, low path length $(L$ and $\lambda)$ and high global efficiency 
$\left(E_{\text {global }}\right)$ express a great ability to integrate information from the whole brain. Optimal brain networks possess both features with a balanced segregation and integration of information processing (Sporns et al., 2004; Bullmore and Sporns, 2009). In contrast, a decrease in clustering coefficient with a decrease in $\mathrm{IQ}$, as found in the patient population, is characteristic for more random networks and can be interpreted as a loss in network organization.

In healthy controls, graph theoretical network parameters are related with level of intelligence (van den Heuvel et al., 2009) supporting the theory that cognitive processes depend on an optimal organization of segregation and integration. To our knowledge, this relation has not been investigated before in patients with epilepsy, who are prone to suffer from a range of cognitive deficits. It is an interesting observation that network topology changes in the presence of epilepsy, and that this alteration is associated with a decline of intellectual abilities.

Of the clinical factors studied, only drug load was associated with network parameters. Antiepileptic drugs (AEDs) inhibit the spread of abnormal neuronal firing to distant sites, thereby suppressing the occurrence of clinical seizures (Rogawski and Loscher, 2004). As AEDs might also have a more generalized suppressive effect (Rogawski and Loscher, 2004), an alteration of neuroexcitability may affect local and global efficiency parameters such as found in the present study. There is too much overlap in AEDs used (Supplemental Table S.3.1) to provide any information on the effect of specific AEDs on network topological parameters, which would be of high interest for future studies. In an epilepsy population, it is complicated to disentangle the effects of AED use and epilepsy per se on network parameters. It would therefore be interesting to investigate whether AED use in other patient populations than those with epilepsy (e.g. in patients with migraine or neuropathic pain) is also associated with change of network parameters.

Although no other clinical factor could be identified to influence the network characteristics, this does not imply that no relation exists. For example, decreased local clustering and small-worldness within the epileptogenic temporal lobe has been associated with longer duration of temporal lobe epilepsy (van Dellen et al., 2009). Moreover, clinical epilepsy factors cannot be viewed as totally independent factors. For example, drug load is likely to increase when patients do not achieve seizure control. In that case, higher drug load can be a marker for a more severe form of epilepsy. Maybe it is not the seizures as such that disturb the cerebral networks, but simply the epilepsy itself. This could imply that there is a shared mechanism leading to both epilepsy and a disruption of cerebral networks that is associated with cognitive decline.

Network parameters may obtain a role in identifying patients at risk for developing cognitive problems. If clinical factors can be identified, this can be beneficial in decision making: for example, patients with advantageous network organization may need less stringent seizure control than patients with disadvantageous network organization. Additionally, network topology might be a more sensitive marker for 
disease progression: if network parameters change before intellectual decline can be measured (normally clinically relevant decline can be observed only after intervals of several years), this may also call for more strict treatment of seizures, and/or earlier referral for epilepsy surgery. Finally, network topological characteristics might be of value in predicting cognitive outcome after epilepsy surgery. 


\section{REFERENCES}

Achard S, Bullmore E (2007) Efficiency and cost of economical brain functional networks. PLoS Comput Biol 3:e17.

Addis DR, Moscovitch M, McAndrews MP (2007) Consequences of hippocampal damage across the autobiographical memory network in left temporal lobe epilepsy. Brain 130:2327-2342.

Baron RM, Kenny DA (1986) The moderator-mediator variable distinction in social psychological research: Conceptual, strategic, and statistical considerations. Journal of personality and social psychology 51:1173.

Bassett DS, Bullmore E (2006) Small-world brain networks. Neuroscientist 12:512-523.

Bernhardt BC, Bernasconi N, Concha L, Bernasconi A (2010) Cortical thickness analysis in temporal lobe epilepsy: reproducibility and relation to outcome. Neurology 74:1776-1784.

Bettus G, Guedj E, Joyeux F, Confort-Gouny S, Soulier E, Laguitton V, Cozzone PJ, Chauvel P, Ranjeva JP, Bartolomei F, Guye M (2009) Decreased basal fMRI functional connectivity in epileptogenic networks and contralateral compensatory mechanisms. Hum Brain Mapp 30:1580-1591.

Bullmore E, Sporns O (2009) Complex brain networks: graph theoretical analysis of structural and functional systems. Nat Rev Neurosci 10:186-198.

Cheung MC, Chan AS, Chan YL, Lam JM, Lam W (2006) Effects of illness duration on memory processing of patients with temporal lobe epilepsy. Epilepsia 47:1320-1328.

Dupont S, Samson Y, Van de Moortele PF, Samson S, Poline JB, Adam C, Lehericy S, Le Bihan D, Baulac M (2001) Delayed verbal memory retrieval: a functional MRI study in epileptic patients with structural lesions of the left medial temporal lobe. Neuroimage 14:995-1003.

Fisher RS, Vickrey BG, Gibson P, Hermann B, Penovich P, Scherer A, Walker S (2000) The impact of epilepsy from the patient's perspective I. Descriptions and subjective perceptions. Epilepsy Res 41:39-51.

Focke NK, Yogarajah M, Bonelli SB, Bartlett PA, Symms MR, Duncan JS (2008) Voxel-based diffusion tensor imaging in patients with mesial temporal lobe epilepsy and hippocampal sclerosis. Neuroimage 40:728-737.

Helmstaedter C, Kurthen M (2001) Memory and epilepsy: characteristics, course, and influence of drugs and surgery. Curr Opin Neurol 14:211-216.

Hermann B, Seidenberg M, Sears L, Hansen R, Bayless K, Rutecki P, Dow C (2004) Cerebellar atrophy in temporal lobe epilepsy affects procedural memory. Neurology 63:2129-2131.

Humphries MD, Gurney K, Prescott TJ (2006) The brainstem reticular formation is a small-world, not scalefree, network. Proc Biol Sci 273:503-511.

Jokeit H, Kramer G, Ebner A (2005) Do antiepileptic drugs accelerate forgetting? Epilepsy Behav 6:430-432.

Lammers MW, Hekster YA, Keyser A, Meinardi H, Renier WO, van Lier H (1995) Monotherapy or polytherapy for epilepsy revisited: a quantitative assessment. Epilepsia 36:440-446.

Liao W, Zhang Z, Pan Z, Mantini D, Ding J, Duan X, Luo C, Lu G, Chen H (2010) Alter ed functional connectivity and small-world in mesial temporal lobe epilepsy. PLoS One 5:e8525.

Liu Y, Liang M, Zhou Y, He Y, Hao Y, Song M, Yu C, Liu H, Liu Z, Jiang T (2008) Disrupted small-world networks in schizophrenia. Brain 131:945-961.

Maslov S, Sneppen K (2002) Specificity and stability in topology of protein networks. Science 296:910-913.

Meletti S, Benuzzi F, Rubboli G, Cantalupo G, Stanzani Maserati M, Nichelli P, Tassinari CA (2003) Impaired facial emotion recognition in early-onset right mesial temporal lobe epilepsy. Neurology 60:426431. 
Oyegbile TO, Bhattacharya A, Seidenberg M, Hermann BP (2006) Quantitative MRI biomarkers of cognitive morbidity in temporal lobe epilepsy. Epilepsia 47:143-152.

Oyegbile TO, Dow C, Jones J, Bell B, Rutecki P, Sheth R, Seidenberg M, Hermann BP (2004) The nature and course of neuropsychological morbidity in chronic temporal lobe epilepsy. Neurology 62:17361742.

Powell HW, Richardson MP, Symms MR, Boulby PA, Thompson PJ, Duncan JS, Koepp MJ (2007) Reorganization of verbal and nonverbal memory in temporal lobe epilepsy due to unilateral hippocampal sclerosis. Epilepsia 48:1512-1525.

Rogawski MA, Loscher W (2004) The neurobiology of antiepileptic drugs. Nature reviews Neuroscience 5:553-564.

Rubinov M, Sporns O (2010) Complex network measures of brain connectivity: uses and interpretations. Neuroimage 52:1059-1069.

Schoenberg MR, Scott JG, Duff K, Adams RL (2002) Estimation of WAIS-III intelligence from combined performance and demographic variables: development of the OPIE-3. Clin Neuropsychol 16:426437.

Sporns O, Chialvo DR, Kaiser M, Hilgetag CC (2004) Organization, development and function of complex brain networks. Trends Cogn Sci 8:418-425.

Stam CJ, Reijneveld JC (2007) Graph theoretical analysis of complex networks in the brain. Nonlinear Biomed Phys 1:3.

Stam CJ, Jones BF, Nolte G, Breakspear M, Scheltens P (2007) Small-world networks and functional connectivity in Alzheimer's disease. Cereb Cortex 17:92-99.

Thompson PJ, Duncan JS (2005) Cognitive decline in severe intractable epilepsy. Epilepsia 46:1780-1787.

van Dellen E, Douw L, Baayen JC, Heimans JJ, Ponten SC, Vandertop WP, Velis DN, Stam CJ, Reijneveld JC (2009) Long-term effects of temporal lobe epilepsy on local neural networks: a graph theoretical analysis of corticography recordings. PLoS One 4:e8081.

van den Heuvel MP, Stam CJ, Kahn RS, Hulshoff Pol HE (2009) Efficiency of functional brain networks and intellectual performance. J Neurosci 29:7619-7624.

Van Dijk KRA, Hedden T, Venkataraman A, Evans KC, Lazar SW, Buckner RL (2010) Intrinsic functional connectivity as a tool for human connectomics: theory, properties, and optimization. Journal of neurophysiology 103:297.

Vlooswijk MC, Jansen JFA, Majoie HJM, Hofman PAM, de Krom MCTFM, Aldenkamp AP, Backes WH (2010a) Functional connectivity and language impairment in cryptogenic localization-related epilepsy. Neurology.

Vlooswijk MC, Jansen JF, de Krom MC, Majoie HM, Hofman PA, Backes WH, Aldenkamp AP (2010b) Functional MRI in chronic epilepsy: associations with cognitive impairment. Lancet Neurol.

Waites AB, Briellmann RS, Saling MM, Abbott DF, Jackson GD (2006) Functional connectivity networks are disrupted in left temporal lobe epilepsy. Ann Neurol 59:335-343.

Watts DJ, Strogatz SH (1998) Collective dynamics of 'small-world' networks.[see comment]. Nature 393:440442.

Weber B, Wellmer J, Schur S, Dinkelacker V, Ruhlmann J, Mormann F, Axmacher N, Elger CE, Fernandez G (2006) Presurgical language $\mathrm{fMRI}$ in patients with drug-resistant epilepsy: effects of task performance. Epilepsia 47:880-886

Wechsler D (1997) Manual for the Wechsler Adult Intelligence Scale-III. San Antonio: The Psychological Corporation.

Zhang Z, Lu G, Zhong Y, Tan Q, Liao W, Chen Z, Shi J, Liu Y (2009) Impaired perceptual networks in temporal lobe epilepsy revealed by resting fMRI. J Neurol 256:1705-1713. 


\section{SUPPLEMENTARY DATA}

\section{Premorbid FSIQ estimate calculation}

Based on the OPIE-3(4ST) from the Oklahoma Premorbid Intelligence Estimate for the WAIS-III Calculation Worksheet (Schoenberg et al., 2002).

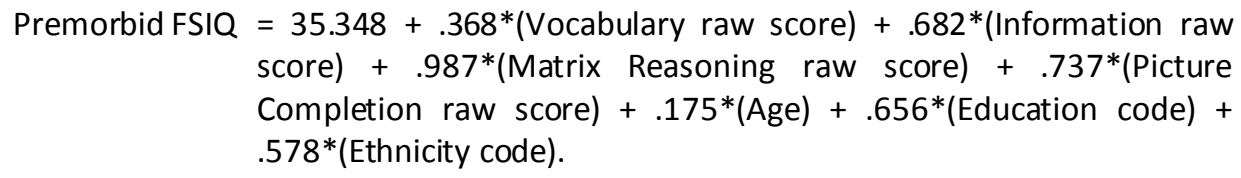

Coding variables:

$\begin{array}{lll}\text { Age } & - & \text { in years } \\ \text { Education } & - & 1=0-8 \text { years } \\ & 2=9-11 \text { years } \\ & 3=12 \text { years } \\ & 4=13-15 \text { years } \\ & 5=16+\text { years } \\ & 1=\text { African-American } \\ & 2=\text { Hispanic } \\ \text { Ethnicity } & 3=\text { Other } \\ & 4=\text { Caucasian } \\ & \text { Note that all participants were Caucasian }\end{array}$

\section{Methods}

\section{Parcellation scheme}

The final brain parcellation into 892 regions was derived from the AAL template, consisting of 90 regions. We started by dividing each AAL region into two more or less equally sized regions by principal component analysis. The first principal component (a 3D vector), together with the center of gravity of the voxels within the region (a 3D point), defines a plane in 3D space which divides the region into two subregions according to the maximum spatial variance of the region (e.g. a "stretched" region will be divided along its main longitudinal axis).

Regions were subsequently subdivided with the criterion that a division must not yield a subregion with a size smaller than 125 voxel. The final result was a parcellation of 892 regions with comparable sizes (of at least 125 voxels).

\section{Sparsity}

Functional connection strengths between two areas $i$ and $j$ were obtained by calculating the Pearson correlation coefficient between the filtered time series of those regions. Each element $A_{i, j}$ of the connection matrix $A$ was defined by the 
Pearson correlation coefficient between every possible pair of brain regions. The connection matrix $A$ is a numerical representation of a graph, which is an abstract data structure, consisting of nodes connected by edges. In the graph, a node is related to a brain region and is represented by a row and a column in the connection matrix. An edge in the graph is a connection between brain areas $i$ and $j$, provided that $A_{i, j}>0$. The brain graph of each individual was thresholded to create graphs with an equal number of nodes and edges across subjects (Stam et al., 2007). This was achieved by selecting the $T_{k}$ strongest connections (connections with the highest correlation coefficient and removing all other connections. The threshold value $T_{k}$ was expressed as a sparsity value relating the number of edges maintained in the network to the total number of edges possible $\left(\mathrm{N}^{2}-\mathrm{N}\right)$, where $\mathrm{N}$ is the number of nodes (brain regions) in the network (Achard and Bullmore, 2007). Let $T_{k}$ be the number of edges maintained in the network, then the sparsity is defined as:

$$
\text { sparsity }=\left(N^{2}-N-T_{k}\right) /\left(N^{2}-N\right)
$$

Theoretically, sparsity can range between 0 (a fully connected graph) and 1 (no edges). A low sparsity value (many connections in the network) implies that connections with a low correlation coefficient are also allowed to exist in the network, which might yield some false positive connections. With a high sparsity value, only edges with the highest correlation coefficients (i.e. strongest connections) remain. As a consequence, edges with intermediate correlation coefficients might be removed albeit being biologically plausible.

\section{Network metrics}

The degree $k$ of node $i$ is the number of connections to other areas:

$$
k=\sum_{j=1, j \neq i}^{N} A_{i . j},
$$

where $N$ is the total number of nodes (i.e. considered brain regions) in the graph.

The graph theoretical metrics characteristic path length $(L)$ and cluster coefficient $(C)$ as well as local and global efficiency were calculated to perform analysis on the constructed brain graphs.

Characteristic path length is defined as the average geodesic distance, in number of edges, connecting any two nodes in the graph:

$$
L=\frac{1}{N(N-1)} \sum_{i=1}^{N} \sum_{j=1, i \neq j}^{N} d_{i, j},
$$

where $d_{i, j}$ is the length of the shortest path between nodes $i$ and $j$. The characteristic path length is a measure of how well connected a network is. Small characteristic path length indicates an average short distance between any two nodes, i.e. they can be reached through a small number of steps. 
The cluster coefficient is defined as the number of actual edges connecting the neighbours of a node divided by the maximum number of edges possible between neighbouring nodes,

$$
C=\frac{1}{N} \sum_{i=1}^{N} \frac{\sum_{j=1}^{N} \sum_{m=1}^{N} A_{i, j} A_{j, m} A_{m, i}}{k_{i}\left(k_{i}-1\right)}
$$

where $i, j$ and $m$ are summation indices. The cluster coefficient of a network is a measure of how many local clusters exist in the network. A high cluster coefficient indicates that the neighbours of a node are often also directly connected to each other, i.e. they form a cluster. Parameters related to characteristic path length are global efficiency $\left(E_{\text {global }}\right)$ and local efficiency $\left(E_{\text {local }}\right)$. $E_{\text {global }}$ is defined as the average inverse shortest path length, $E_{\text {local }}$ is defined as the mean of the global efficiencies of subgraphs consisting of the immediate neighbours of a particular node. These parameters may be meaningfully computed on disconnected graphs and are a superior measure of integration according to some authors (Achard and Bullmore, 2007).

\section{Random networks}

To be able to determine whether a network has small-world properties, the values of $L$ and $C$ must be scaled to values from generated random networks (Watts and Strogatz, 1998). Small world networks are characterized by having $L$ close to random: $\lambda=L / L_{\text {random }} \approx 1$, but with $C$ higher than random: $\gamma=C / C_{\text {random }}>1$. The value $\sigma=\gamma / \lambda$ can be used to signify the 'small-worldness' of a network and is typically larger than 1 for small-world networks (Humphries et al., 2006).

Random networks were generated by considering each existing edge in the original network between the nodes $i$ and $j, e_{i, j}$ and connecting it to another randomly chosen node $j 2$, with the condition that $e_{i, j 2}$ was not present in the original network (Maslov and Sneppen, 2002). This process ensures that the node degree and node distribution of the random network is similar to the original network. Network parameters were calculated with routines from the brain connectivity toolbox (Rubinov and Sporns, 2010). 
A

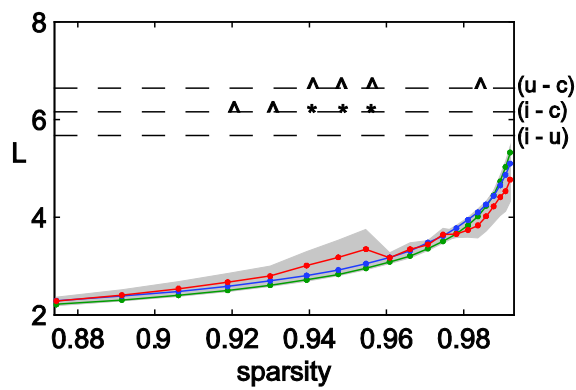

B

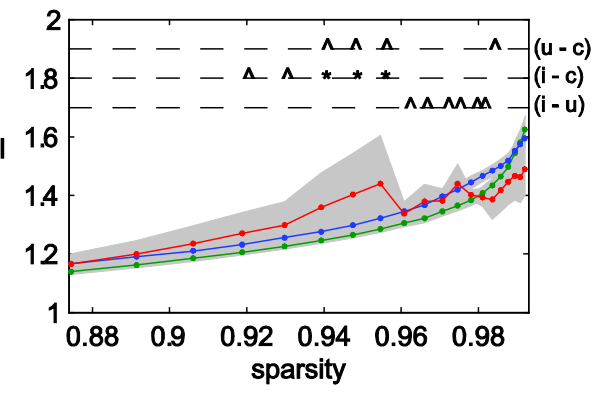

Figure S.3.1 Path length and lambda parameters for the whole brain network including cognitively impaired patients. Networks of the impaired patient group (red lines), the unimpaired patient group (blue lines) and the control group (green lines) as function of sparsity for (A) path length and (B) lambda. Grey areas indicate standard error of the mean for each of the analyzed groups. The dashed lines represent significant group differences (annotated with an asterisk) or trends (annotated with a triangle) towards group differences for the unimpaired versus the control group ( $u-c$, top line), the impaired versus the control group (i-c, middle line) and the impaired versus unimpaired group ( $\mathrm{i}-\mathrm{u}$, bottom line).
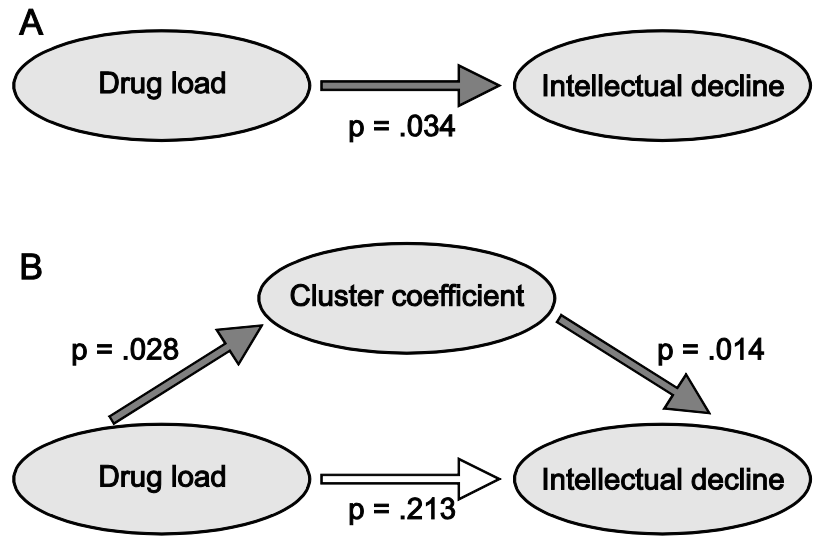

Figure S.3.2 The mediator analysis. (A) Drug load as a predictor of intellectual decline when graph theoretical network parameters are not considered. (B) When graph theoretical network parameters are considered, the cluster coefficient $(C)$ mediates the relation between drug load and intellectual decline. $P$-values represent the values for sparsity value 0.97 . 
Table S.3.1 Clinical characteristics of patients and controls

\begin{tabular}{|c|c|c|c|c|c|c|c|c|c|c|}
\hline 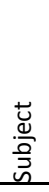 & 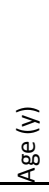 & 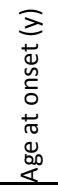 & 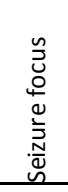 & 心్ & 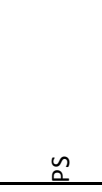 & $\begin{array}{l}\text { D } \\
\frac{0}{00} \\
\frac{0}{30} \\
\vdots \\
\end{array}$ & 峞 & $\frac{\sigma}{\tilde{}}$ & $\underline{\Xi}$ & 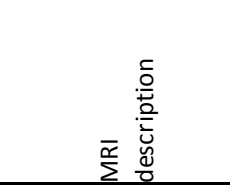 \\
\hline \multicolumn{11}{|c|}{ Patients } \\
\hline 1 & 49 & 43 & LT & 2 & $11-20$ & 1.40 & LTG, VPA & 78 & -16 & no abnormalities \\
\hline 2 & 26 & 13 & RT & 0 & $>100$ & 1.79 & LEV, LTG & 98 & -8 & no abnormalities \\
\hline 3 & 34 & 6 & $\mathrm{LF}$ & 6 & $>100$ & 3.74 & PHT, LEV, LTG & 108 & -9 & no abnormalities \\
\hline 4 & 46 & 13 & BT & 40 & $>100$ & 1.70 & $\mathrm{CBZ}, \mathrm{GBP}$ & 61 & -9 & no abnormalities \\
\hline 5 & 41 & 13 & LF & 24 & $31-40$ & 3.33 & CBZ, CLB, LEV & 77 & -24 & no abnormalities \\
\hline 6 & 23 & 4 & $\mathrm{LF}$ & 50 & $>100$ & 4.80 & CBZ, LEV, LTG & 79 & -10 & $\begin{array}{l}\text { cystic enlargement } \\
\text { pineal gland }\end{array}$ \\
\hline 7 & 22 & 13 & $\mathrm{BF}$ & 35 & none & 1.38 & LEV & 85 & -12 & no abnormalities \\
\hline 8 & 55 & 21 & $\mathrm{BF}$ & 33 & none & 1.67 & VPA & 70 & -3 & no abnormalities \\
\hline 9 & 30 & 20 & LFT & 4 & $31-40$ & 1.45 & LEV, LTG & 89 & -17 & no abnormalities \\
\hline 10 & 46 & 40 & RFT & 0 & $21-30$ & 1.33 & VPA & 88 & -18 & no abnormalities \\
\hline 11 & 49 & 40 & RT & 2 & $51-100$ & 1.97 & CBZ, CLB, VPA & 100 & -15 & no abnormalities \\
\hline 12 & 50 & 34 & LT & 3 & $>100$ & 3.61 & CBZ, LEV, LTG & 95 & -9 & no abnormalities \\
\hline 13 & 28 & 21 & LT & 10 & $>100$ & 0.85 & $\mathrm{CBZ}, \mathrm{CZP}$ & 106 & -3 & $\begin{array}{l}\text { atrophy left } \\
\text { temporal lobe }\end{array}$ \\
\hline 14 & 59 & 23 & LT & 3 & $31-40$ & 1.74 & CBZ, LTG & 86 & -3 & MTS left \\
\hline 15 & 24 & 8 & LF & 17 & $1-10$ & 2.51 & CBZ, LTG & 95 & -4 & no abnormalities \\
\hline 16 & 61 & 48 & BFT & 4 & none & 0.40 & CBZ & 111 & -5 & no abnormalities \\
\hline 17 & 23 & 8 & LT & 500 & $51-100$ & 1.94 & CBZ, LTG & 98 & -8 & no abnormalities \\
\hline 18 & 56 & 51 & LFT & 0 & $41-50$ & 1.20 & OCBZ & 113 & -10 & no abnormalities \\
\hline 19 & 63 & 56 & LFT & 1 & $>100$ & 1.20 & OCBZ & 101 & 0 & no abnormalities \\
\hline 20 & 22 & 10 & $\mathrm{BFT}$ & 1 & $>100$ & 2.95 & LEV, LTG & 96 & -10 & no abnormalities \\
\hline 21 & 36 & 14 & RFT & 3 & $>100$ & 1.80 & OCBZ & 97 & -4 & no abnormalities \\
\hline 22 & 51 & 4 & BT & 30 & $>100$ & 2.70 & CBZ, CLB, LEV & 95 & -6 & MTS left \\
\hline 23 & 38 & 34 & LT & 2 & $1-10$ & 0.90 & OCBZ & 97 & 0 & no abnormalities \\
\hline 24 & 45 & 33 & LF & 3 & none & 0.60 & OCBZ & 121 & -2 & no abnormalities \\
\hline 25 & 31 & 7 & LFT & 8 & $1-10$ & 0.20 & VPA & 94 & -7 & no abnormalities \\
\hline 26 & 29 & 29 & RFT & 0 & $11-20$ & 1.43 & LTG & 80 & -11 & $\begin{array}{l}\text { venous angioma } \\
\text { right frontal }\end{array}$ \\
\hline 27 & 45 & 14 & $\mathrm{BF}$ & 35 & none & 2.29 & CLB, LTG & 121 & -3 & no abnormalities \\
\hline 28 & 41 & 39 & BFT & 3 & $11-20$ & 0.80 & $\mathrm{CBZ}$ & 105 & -8 & no abnormalities \\
\hline 29 & 30 & 13 & LFT & 0 & $>100$ & 3.03 & CLB, OCBZ, VPA & 79 & -10 & no abnormalities \\
\hline 30 & 36 & 18 & $\mathrm{BF}$ & 35 & $1-10$ & 2.20 & $\mathrm{CBZ}, \mathrm{PHT}$ & 83 & -16 & no abnormalities \\
\hline 31 & 60 & 39 & $\mathrm{BF}$ & 0 & $>100$ & 1.94 & CBZ, LTG & 101 & -8 & no abnormalities \\
\hline 32 & 44 & 25 & RT & 6 & $1-10$ & 3.25 & PHT, OCBZ & 101 & -11 & $\begin{array}{l}\text { increased FLAIR } \\
\text { signal right HC, } \\
\text { normal HC volumes }\end{array}$ \\
\hline 33 & 35 & 23 & $\mathrm{BF}$ & 10 & $>100$ & 1.00 & CLB & 128 & 10 & no abnormalities \\
\hline 34 & 27 & 13 & $\mathrm{BF}$ & 3 & none & 0.40 & CBZ & 129 & 8 & no abnormalities \\
\hline 35 & 52 & 12 & $\mathrm{BF}$ & 4 & $>100$ & 1.30 & $\mathrm{CBZ}, \mathrm{PB}$ & 97 & -11 & no abnormalities \\
\hline 36 & 26 & 10 & RFT & 1 & $1-10$ & 0.50 & CLB & 104 & -7 & no abnormalities \\
\hline 37 & 33 & 4 & RFT & 3 & $11-20$ & 2.00 & CLB, LTG & 91 & -17 & no abnormalities \\
\hline 38 & 58 & 29 & LT & 20 & $>100$ & 2.14 & CLB, LTG, OCBZ & 91 & -17 & MTS left \\
\hline 39 & 43 & 27 & $\mathrm{RF}$ & 20 & $1-10$ & 0.90 & CLB, VPA & 86 & -7 & no abnormalities \\
\hline 40 & 37 & 29 & BT & 30 & $1-10$ & 1.20 & CLB, VPA & 84 & -12 & no abnormalities \\
\hline 41 & 55 & 26 & RT & 6 & $41-50$ & 0.40 & $\mathrm{CBZ}$ & 109 & -5 & no abnormalities \\
\hline
\end{tabular}




\begin{tabular}{|c|c|c|c|c|c|c|c|c|c|c|}
\hline 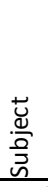 & 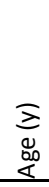 & 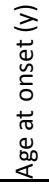 & 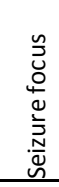 & 氜 & $\simeq$ & $\begin{array}{l}0 \\
\frac{\pi}{0} \\
\frac{0}{00} \\
30 \\
0 \\
\end{array}$ & 岂 & $\frac{\sigma}{\sim}$ & $\underline{\Xi}$ & 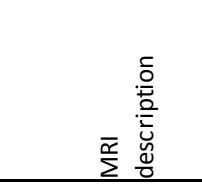 \\
\hline \multicolumn{11}{|c|}{ Healthy controls } \\
\hline 42 & 53 & NA & NA & NA & NA & NA & NA & 111 & -10 & no abnormalities \\
\hline 43 & 52 & NA & NA & NA & NA & NA & NA & 115 & -6 & no abnormalities \\
\hline 44 & 45 & NA & NA & NA & NA & NA & NA & 106 & -8 & no abnormalities \\
\hline 45 & 51 & NA & NA & NA & NA & NA & NA & 126 & 1 & no abnormalities \\
\hline 46 & 32 & NA & NA & NA & NA & NA & NA & 107 & -16 & no abnormalities \\
\hline 47 & 28 & NA & NA & NA & NA & NA & NA & 88 & -15 & no abnormalities \\
\hline 48 & 28 & NA & NA & NA & NA & NA & NA & 108 & -3 & no abnormalities \\
\hline 49 & 46 & NA & NA & NA & NA & NA & NA & 108 & -7 & $\begin{array}{l}\text { variant septum } \\
\text { pellucidum }\end{array}$ \\
\hline 50 & 32 & NA & NA & NA & NA & NA & NA & 112 & -6 & no abnormalities \\
\hline 51 & 52 & NA & NA & NA & NA & NA & NA & 140 & 12 & no abnormalities \\
\hline 52 & 23 & NA & NA & NA & NA & NA & NA & 138 & 15 & no abnormalities \\
\hline 53 & 48 & NA & NA & NA & NA & NA & NA & 112 & -8 & no abnormalities \\
\hline 54 & 24 & NA & NA & NA & NA & NA & NA & 122 & 5 & no abnormalities \\
\hline 55 & 56 & NA & NA & NA & NA & NA & NA & 129 & 3 & no abnormalities \\
\hline 56 & 47 & NA & NA & NA & NA & NA & NA & 105 & -9 & no abnormalities \\
\hline 57 & 47 & NA & NA & NA & NA & NA & NA & 147 & 13 & no abnormalities \\
\hline 58 & 46 & NA & NA & NA & NA & NA & NA & 110 & -13 & no abnormalities \\
\hline 59 & 54 & NA & NA & NA & NA & NA & NA & 91 & -11 & no abnormalities \\
\hline 60 & 18 & NA & NA & NA & NA & NA & NA & 117 & 4 & no abnormalities \\
\hline 61 & 21 & NA & NA & NA & NA & NA & NA & 112 & 0 & no abnormalities \\
\hline 62 & 51 & NA & NA & NA & NA & NA & NA & 105 & -12 & no abnormalities \\
\hline 63 & 58 & NA & NA & NA & NA & NA & NA & 91 & -6 & no abnormalities \\
\hline 64 & 18 & NA & NA & NA & NA & NA & NA & 105 & -6 & no abnormalities \\
\hline
\end{tabular}

Abbreviations: $y$ = years; SGS = total number of secondarily generalized seizures; $P S=$ total number of partial seizures; FSIQ = full-scale IQ; IQ $\Delta=I Q$ discrepancy score $\left(I Q_{\text {actual }}-I_{\text {premorbid estimate }}\right) ; L=I$ eft; $R=$ right; $T$ = temporal; $\mathrm{F}=$ frontal; $\mathrm{FT}=$ frontotemporal; $\mathrm{B}=$ bilateral; $\mathrm{NA}=$ not applicable; $\mathrm{MTS}=$ mesiotemporal sclerosis; $\mathrm{HC}=$ hippocampus. $\mathrm{AED}=$ antiepileptic drugs; $\mathrm{CBZ}=$ carbamazepine; $\mathrm{CLB}$ = clobazam; $\mathrm{CZP}=$ clonazepam; $\mathrm{PB}$ = fenobarbital; $\mathrm{GBP}=$ gabapentin; $\mathrm{LEV}=$ levetiracetam; LTG = lamotrigine; $\mathrm{OCBZ}=$ oxcarbazepine; $\mathrm{PHT}$ = phenytoin; VPA = valproic acid. Drug load was calculated by the ratio of prescribed daily dose to defined daily dose (Lammers et al., 1995). 


\section{CHAPTER 4}

\section{White matter network abnormalities are associated with cognitive decline in chronic epilepsy}




\section{ABSTRACT}

Patients with chronic epilepsy frequently display cognitive comorbidity and might have widespread network abnormalities outside the epileptic zone, which might affect a variety of cognitive functions and global intelligence. We aimed to study the role of white matter connectivity in cognitive comorbidity. Thirty-nine patients with nonsymptomatic localization-related epilepsy and varying degrees of cognitive impairment and 23 age-matched healthy controls were included. Whole brain white matter networks were constructed from fiber tractography. Weighted graph theoretical analysis was performed to study white matter network abnormalities associated with epilepsy and cognition. Patients with severe cognitive impairment showed lower clustering (a measure of brain network segregation) and higher path length (a measure of brain network integration) compared with the healthy controls and patients with little or no cognitive impairment, whereas whole brain white matter volume did not differ. Correlation analyses revealed that IQ and cognitive impairment were strongly associated with clustering and path lengths. This study revealed impaired white matter connectivity, associated with cognitive comorbidity in patients with chronic epilepsy. As whole brain white matter volumes were preserved in the patient group, our results suggest an important role for the network topology rather than volumetric changes, in epilepsy with cognitive decline. 


\section{INTRODUCTION}

Patients with chronic epilepsy frequently display co-morbid cognitive problems, ranging from memory deficits, mental slowing (Elger et al., 2004) and language problems (Vlooswijk et al., 2010), to global cognitive deterioration (Oyegbile et al., 2004). Patients with epilepsy often have lower intelligence levels than expected compared to healthy controls (Helmstaedter and Kockelmann, 2006; Bonelli et al., 2010) even in studies that exclude symptomatic epilepsy (Hermann et al., 1995).

There is recent consensus that many cognitive functions result from the concerted interaction between brain areas in large-scale networks (Bressler and Menon, 2010; Deary et al., 2010). The functioning of these networks is constrained by the organization of the associated axonal bundles of the white matter (WM). Magnetic resonance imaging (MRI) studies on WM and functional networks (Li et al., 2009; van den Heuvel et al., 2009) furthermore show that network efficiency plays an important role in intelligence. Therefore any abnormality in the axonal network organization, associated with epilepsy, may explain cognitive decline in a much more sensitive way than changes in specific brain structures, as the location of the affected structures may vary from patient to patient.

The extent to which brain abnormalities manifest beyond the epileptic zone is currently a matter of debate (Meador and Hermann, 2010). For instance, there is growing evidence from Diffusion Tensor Imaging (DTI) that micro-structural WM abnormalities are present outside the epileptic focus in temporal lobe epilepsy (TLE) (Focke et al., 2008b; Meng et al., 2010; Riley et al., 2010), and that distant fiber bundles are affected (Powell et al., 2007; Yogarajah et al., 2008) and associated with cognitive co-morbidity (Diehl et al., 2008; McDonald et al., 2008; Yogarajah et al., 2008; Riley et al., 2010).

For cryptogenic epilepsy, the imaging of WM abnormalities focused on predefined brain regions or connections gives rise to interpretational ambiguities because the precise anatomical location of the epileptic zone often remains uncertain and may vary between patients. A method that adequately deals with these problems is graph theoretical analysis (Stam and Reijneveld, 2007; Bullmore and Sporns, 2009). Graph theoretical analysis typically captures topological properties of the brain network in a few summary measures, which provide information on the amount of segregation and integration among brain regions. With graph theoretical analysis it is possible to investigate the whole brain network, dividing it into a large number of regions (nodes) with an even larger number of possible connections (edges). It can then be calculated how the network is organized. One distinction that can be made is between a 'smallworld' and a random topology. A 'small-world' network is characterized by a high degree of local clustering and short path lengths that globally link all the regions of the network. On the contrary, in a random topology all links in the graph have an equal probability connecting any two nodes. This methodology has recently been shown to provide more sensitive measurements than conventional DTI indices in stroke (Crofts 
et al., 2011) and in schizophrenia (van den Heuvel et al., 2010; Zalesky et al., 2010c). Graph theoretical analysis of WM networks has not been performed in epilepsy and might provide valuable insights into the extent and nature of WM network abnormalities and their potential relation with decline in cognitive performance (cognitive co-morbidity).

Our objective was to investigate the integrity of white matter network organization using graph theoretical network analysis in patients with cryptogenic, localization related, epilepsy with fronto-temporal focus in comparison to age matched healthy controls. We investigated to which extent patients with epilepsy show abnormal white matter network properties and whether these relate to cognitive impairment.

\section{MATERIALS AND METHODS}

\section{Study population}

All patients were included from the tertiary referral epilepsy center Kempenhaeghe (Heeze, the Netherlands) and the outpatient clinic neurology of Maastricht University Medical Centre (Maastricht, the Netherlands). Inclusion criteria for the patients were: cryptogenic (i.e. non-symptomatic) localization-related epilepsy with a temporal and/or frontal epileptic focus, no history of status epilepticus and no other underlying disease that could possibly cause cognitive decline. Healthy controls were family members and acquaintances of the patients without a history of brain injury or cognitive problems. Thirty-nine patients (19 males, age $40 \pm 12 \mathrm{y}$ ) and 23 age-matched

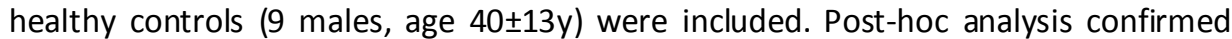
that the patient and healthy control group did not differ in age ( $t$-test, $p=0.65$ ) or group composition of gender (Chi-square, $p=0.48$ ). All subjects underwent a full intelligence (FS-IQ) assessment with the Wechsler Adult Intelligence Scale (WAIS-III) (Wechsler, 1997). Patients were considered to be either cognitively impaired or unimpaired based on an estimation of their pre-morbid IQ (Schoenberg et al., 2002). An estimate of pre-morbid intelligence levels was made according to the formula proposed by Schoenberg et al. (Schoenberg et al., 2002), which is based on the observation that the subtests Vocabulary, Information, Matrix Reasoning, and Picture Completion are relatively resistant to neurological insult (Wechsler, 1997). Intelligence discrepancy scores were calculated by subtracting pre-morbid and full-scale IQ (FSIQ) scores. Patients with a difference between FS-IQ and pre-morbid IQ lower than any differences recorded in the healthy control group were categorized as cognitively impaired. This resulted in a subgroup of $n=7$ ( 4 males) cognitively impaired patients. This subgroup had comparable age $(41 \pm 10 y)$ to the healthy control and non-impaired groups. Patient and epilepsy characteristics, including drug load, seizure frequency and age at onset were collected as described in (Vlooswijk et al., 2010). An overview of the study population is given in Table 4.1. 
Table 4.1 Demographics and epilepsy parameters. Notation : mean (standard deviation).

\begin{tabular}{lccc}
\hline & $\begin{array}{c}\text { Controls } \\
(\mathrm{n}=23)\end{array}$ & $\begin{array}{c}\text { Non-impaired patients } \\
(\mathrm{n}=32)\end{array}$ & $\begin{array}{c}\text { Impaired patients } \\
(\mathrm{n}=7)\end{array}$ \\
\hline Gender (M/F) & $9 / 14$ & $15 / 17$ & $4 / 3$ \\
Age (yr) & $40.4(13.5)$ & $40.8(13.1)$ & $41.8(9.8)$ \\
FS-IQ & $113.2(15.0)$ & $97.6(15.5)$ & $85.2(5.9)$ \\
IQ discrepancy & $-3.6(8.8)$ & $-6.5(5.3)$ & $-17.8(2.8)$ \\
Age at onset (yr) & $\mathrm{n} / \mathrm{a}$ & $23.1(14.1)$ & $23.8(14.2)$ \\
Duration of epilepsy (yr) & $\mathrm{n} / \mathrm{a}$ & $17.6(11.5)$ & $18.0(10.7)$ \\
Drug load & $\mathrm{n} / \mathrm{a}$ & $1.64(1.07)$ & $1.98(0.70)$ \\
\hline
\end{tabular}

$\mathrm{FS}-\mathrm{IQ}=$ Full-Scale Intelligence Quotient. $\mathrm{n} / \mathrm{a}=$ not applicable

\section{Image analysis}

Diffusion Tensor Imaging (DTI) measurements were performed in all subjects on a 3 Tesla MRI system (Philips Medical Systems, Achieva). Acquisition parameters for DTI were: 52 contiguous 2-mm thick slices, matrix size $96 \times 96$, pixel size $2 \times 2 \mathrm{~mm}$, TE 62 ms and TR $6600 \mathrm{~ms}$. Images were obtained along 15 non-collinear diffusion directions with a b-value of $800 \mathrm{~s} / \mathrm{mm}^{2}$; one $b=0 \mathrm{~s} / \mathrm{mm}^{2}$ image was acquired. Anatomic reference images were acquired by a T1 weighted 3D-fast gradient echo sequence (Jeukens et al., 2009).

Each data set was spatially co-registered to the $b=0$ image with an affine transformation to correct for head motion and eddy-current distortions utilizing CATNAP (Co-registration, Adjustment, and Tensor-solving, a Nicely Automated Program, version 1.3) software (Farrell et al., 2007). The set of gradient vectors was adjusted according to the rotation of the individual images.

\section{Whole brain white matter volume}

As our network parameters are based on fiber tract volume, we also investigated group differences in white matter volume and the relation between white matter volume relative to total intracranial volume and FS-IQ. White matter volumes were obtained from a probabilistic tissue segmentation on the subjects' T1-weighted images (Zhang et al., 2001).

\section{Region definition}

The Automatic Anatomical Labeling (AAL) atlas was used to define $\mathrm{N}=90$ cortical and sub-cortical regions (Tzourio-Mazoyer et al., 2002). The AAL volumes of interest (VOI) were then transformed to DTI space of every individual, by first applying a nonlinear 
transformation between standard space and T1 space followed by an affine transformation from T1 space to DTI space (Ashburner and Friston, 1999).

\section{Tractography}

Probabilistic tractography was performed in original DTI space according to previously described methods (Parker et al., 2003) using the CAMINO toolbox (Cook et al., 2006). The Probabilistic Index of Connectivity (PICo) algorithm was used to apply the fiber tracking from the defined VOIs in the original space. This method models uncertainty, due to noise or crossing of fibers, in fiber orientation with probability density functions (PDFs). This method is based on streamline tractography, but incorporates Monte Carlo sampling methods to generate maps of connection probabilities from selected seed regions. Tracts were terminated when a curvature threshold of $60^{\circ}$ over one voxel was encountered (Toosy et al., 2004). Tractography was performed in original DTI space.

For all subjects, an individual cerebrum mask was created by applying the Brain Extraction Tool (Smith, 2002) on the $b=0$ diffusion image. This mask was used to limit the tractography to within the cerebrum. Only voxels on the boundary of the greywhite matter interface were used for initiating tractography. Using the T1 tissue segmentation, the grey-white matter boundary was defined by selecting voxels where the joint tissue probability $(T)$ for grey and white matter was above a certain threshold ( $T>0.2)$ (Vaessen et al., 2010). The results were transformed from the subjects' T1weighted image space to diffusion image space, using a rigid body transformation (Smith et al., 2004). One thousand tracts were initiated from each voxel, leading to an average of 23 million tracts per brain. Subsequently, for each pair of regions, the subset of tracts connecting these two regions were identified from the set of tracts of the whole brain tractography. As an additional noise filter, voxels that were traversed by fewer than 50 tracts were eliminated from the analysis. Exploratory analysis revealed that voxels with $<50$ tracts (i.e. $<5 \%$ of the number of streamlines generated per seed voxel) were widespread throughout the brain and often did not seem to represent plausible anatomical connectivity and were therefore considered noise.

\section{Network construction}

Structural connection strengths between two regions $i$ and $j$ were obtained by calculating the total volume of the voxels within the fiber tracts connecting those regions and scaling these by the total intracranial volume. This scaling is necessary because total brain volume is a confounder for measures based upon tract volume. Another plausible option would be to correct for the total volume traversed by the generated fiber tracts. However, an additional analysis revealed that these two measures are strongly correlated (Peason's $r=0.99$ ), yielding nearly equal corrections. The connection matrix $A$ was formed by calculating the structural connection strength between all pairs of brain regions. The matrix $A$ is a numerical representation of a 
graph $(G)$, which is an abstract data structure, consisting of nodes connected by edges. In the graph, a node is related to a brain region and represents a row or column in the connection matrix. An edge in the graph is a connection between brain areas $i$ and $j\left(A_{i, j}\right)$.

The total number of edges $(K)$, regardless of weight, found in each individual subject was investigated for group differences and associations with FS-IQ. We also investigated all connections of the connectivity matrices for group differences in edgeweights (tract volume) and for associations with FS-IQ.

\section{Graph theoretical analysis}

Graph theoretical network analysis was used to investigate whether the brain structural network parameters differed between epilepsy patients and the healthy control group and for possible correlations with cognition and epilepsy parameters.

Critically, as the number of edges in a network is a strong confounder for subsequent network parameters, it is best to compare networks where the number of edges is kept constant over subjects (van Wijk et al., 2010). Moreover, with tractography the presence or absence of a fiber tract might be hard to interpret, especially when the presence varies from subject to subject. Therefore we chose the following approach: only fiber tracts that were found in every subject were allowed as edges in the final connectivity matrices. As a result all individuals had exactly the same set of edges in their networks. The use of a fixed set of edges implies that any differences in network parameters between subjects are solely due to differences in edge weights as opposed to differences in binary connectivity patterns per se.

The graph theoretical parameters weighted characteristic path length $(L)$ and weighted cluster coefficient $(C)$ were calculated to perform analysis on the constructed volume weighted brain graphs. The weighted characteristic path length (Rubinov and Sporns, 2010) is defined as the average of the shortest paths connecting any two nodes in the graph:

$$
L=\frac{1}{N(N-1)} \sum_{i, j \in G, i \neq j} w_{i, j},
$$

where $w_{i, j}$ is the sum of weights (i.e. tract volume) of the shortest weighted path between nodes $i$ and $j$. The characteristic path length is a measure of how well connected a network is. In an un-weighted graph, a small characteristic path length indicates that, on average, any two nodes are connected through only a few edges. In the case of a volume weighted network a larger tract volume will decrease the distance (i.e. the connection strength) between two nodes, and thus a short path length indicates that, on average, any two nodes are connected by one or several large fibers bundles. The weighted cluster coefficient (Onnela et al., 2005) is defined as:

$$
C=\frac{1}{N} \sum_{i} \frac{\sum_{j, m}\left(A_{i, j} A_{j, m} A_{m, i}\right)^{1 / 3}}{k_{i}\left(k_{1}-1\right)}
$$


The (un-weighted) cluster coefficient of a network is a measure of cliqueness, i.e. sets of nodes that are highly interconnected. For a volume weighted graph, the cluster coefficient is high when the direct neighbors of a node are also interconnected and have relatively large tract volumes.

Network parameters were calculated with routines from the brain connectivity toolbox (Rubinov and Sporns, 2009). The entire processing pipeline is visualized in Figure 4.1.

The weighted graph metrics are in part dependent on the average weight of the connectivity matrix. Therefore, the mean matrix weight was tested for group differences and associations with FS-IQ and the graph metrics were tested with and without adjustment for average matrix weight.

\section{Statistics}

Group differences in parameters were tested using a Student's t-test, associations between network parameters, connections and other subject related variables were tested with Pearson's correlation coefficient and partial correlation coefficients.

\section{RESULTS}

\section{Whole brain white matter volume}

The FS-IQ of the impaired patient group (mean \pm SD, 85.2 \pm 6.0 ) was significantly lower compared to the healthy control group $(113.2 \pm 15.0, p<0.001)$ and the non-impaired patient group $(97.6 \pm 15.6, p<0.05)$. The non-impaired patient group had significantly lower FS-IQ compared to the control group $(p<0.001)$, see Table 4.1.

White matter volumes (as a fraction of total intracranial volume) did not differ significantly between the impaired patient group $(0.471 \pm 0.05)$, the healthy control $(0.481 \pm 0.06, p=0.73)$ and the non-impaired patient group $(0.461 \pm 0.10, p=0.78)$. White matter volume was significantly correlated with $C(r=-0.39, p<0.02)$ and $L(r=0.46$, $p<0.005)$. No association was found between whole brain white matter volume and FS-IQ ( $p=0.85)$ in the entire patient group (the non-impaired plus the impaired patient group). 


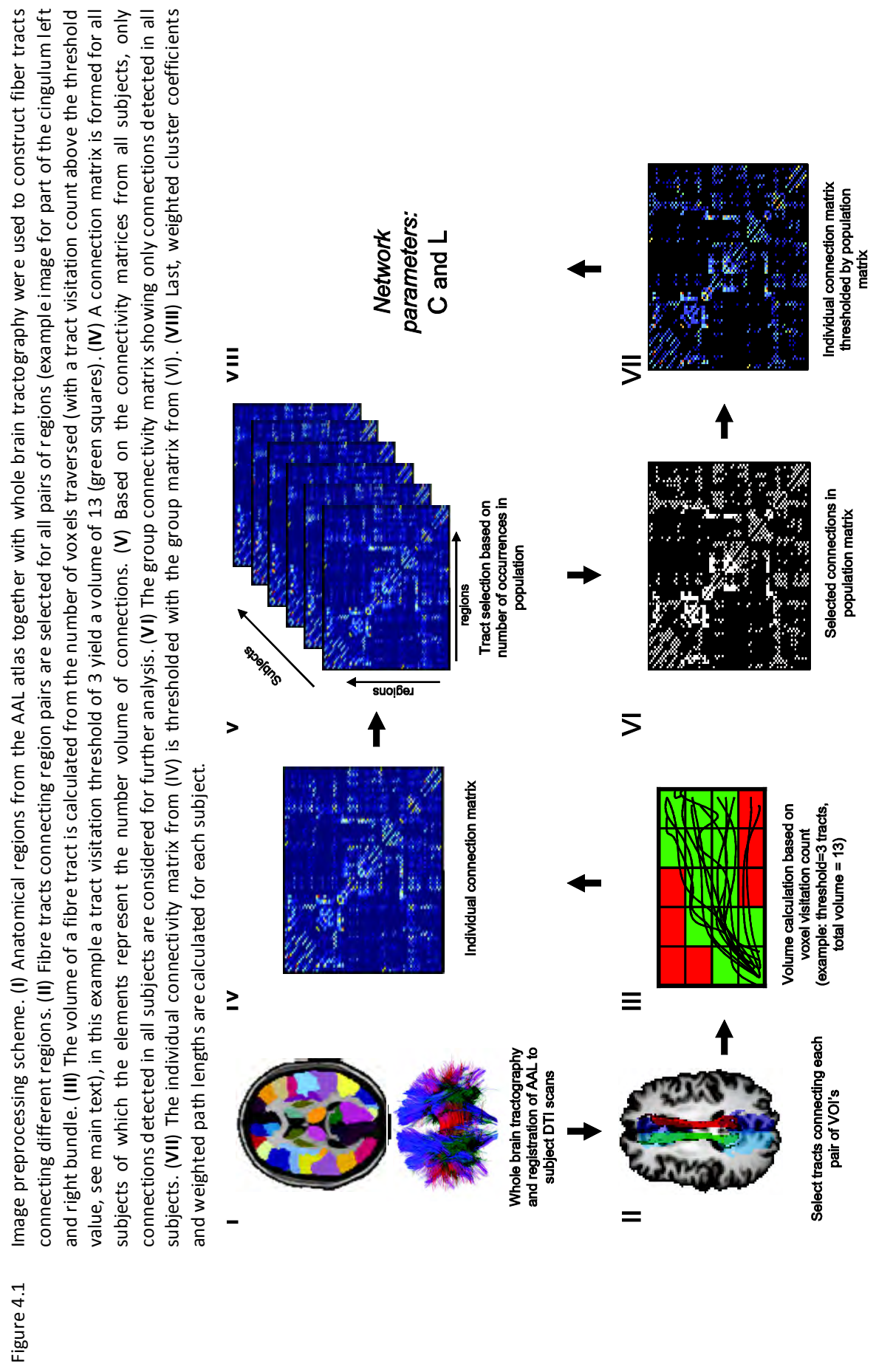




\section{Number of connections and fiber bundle volume}

We found a lower number of reconstructed fiber tracts for the impaired patient group compared to the healthy control group $(p<0.01)$ and the non-impaired patient group $(p<0.04)$, see Figure 4.2A. The number of edges $(K)$ was found to be positively correlated with FS-IQ in the patient group $(r=0.41, p<0.009)$.

Edges showing differences in volume between the different patient groups (at $p<0.05$, uncorrected) were widespread throughout the brain as shown in Figure 4.3. Almost all tracts volumes were smaller for the patient groups. Critically, none of these differences remained significant after FDR ( $q=0.05)$ correction for multiple comparisons (Benjamini and Hochberg, 1995).

The correlation analysis of each fiber tract with FS-IQ in the entire patient group revealed a number of significant correlations ( $p<0.001$, uncorrected) of edges mainly in the left hemisphere, as shown in Figure 3D. These correlations were no longer significant after FDR ( $q=0.05$ ) correction for multiple comparisons.

The mean matrix weight was slightly lower in the non impaired patient group $(305 \pm 53)$ relative to the healthy control group $(322 \pm 39$, n.s.), and even further decreased for the impaired patient group $(269 \pm 42, p=0.01)$. Mean matrix weight was positively correlated with FS-IQ in the entire patient group $(r=0.39, p<0.02)$.

\section{Network analysis}

The final networks consisted of 90 cortical and sub-cortical regions with 1224 edges per subject. The weighted cluster coefficient was significantly lower for the impaired patient group $(0.0153 \pm 0.0023)$ compared to the healthy control group $(0.0182 \pm 0.0032, p<0.04)$ and the non-impaired group $(0.0180 \pm 0.0029, p<0.02)$, see Figure 4.2B. For the path length, the impaired patient group $(69.7 \pm 10.1)$ had significantly higher values compared to the healthy control group $(60.9 \pm 10.0, p<0.05)$ and the non-impaired group $(63.3 \pm 9.6, p<0.04)$, see Figure $4.2 \mathrm{C}$.

Correlation analyses between $C, L, I Q$, pre-morbid $I Q$ and $I Q$ discrepancy were performed in the entire patient group, with and without correction for age and gender. Figure 4.4 shows an overview of the tested variables. Age was found to be predictive for both $C$ and $L$. An increase in age was associated with a decrease in $C$ ( $r=-$ $0.40, p<0.01)$ and an increase in $L(r=0.33, p<0.04)$.

Weighted cluster coefficient $C$ was positively associated with FS-IQ when corrected for age and gender $(r=0.58, p<0.001)$, this was also significant without corrections $(r=0.39$, $p<0.016)$. Path length was significantly and negatively associated with FS-IQ when corrected for age and gender $(r=-0.57, p<0.001)$, and was also significant without corrections $(r=-0.40, p<0.011)$. When also correcting for mean matrix weight in addition to age and gender, $C$ was still correlated with FS-IQ $(r=0.40, p=0.01)$ as was $L$ $(r=-0.41, p=0.01)$. 

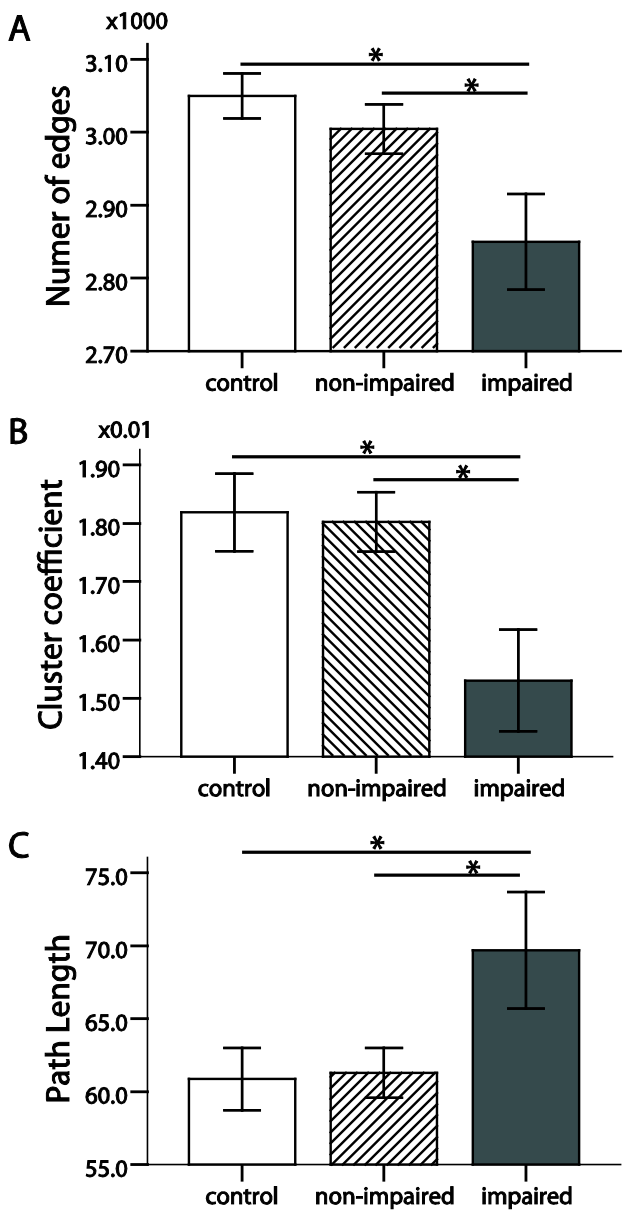

Figure 4.2 Between group comparison of network parameters. (A) Number of edges of the unthresholded networks, (B) weighted cluster coefficient and (C) weighted path length of the thresholded networks in epilepsy patients with and without cognitive impairment and healthy controls. Error bars indicate the standard error of the mean. Asterisk denote a significant difference $(p<0.05)$. 
A
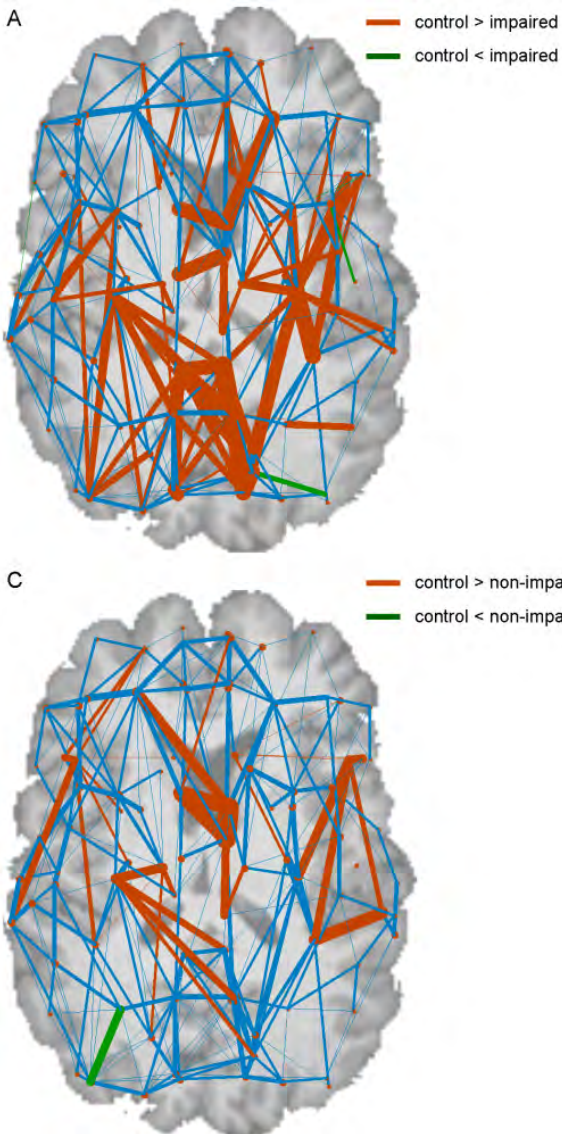

B

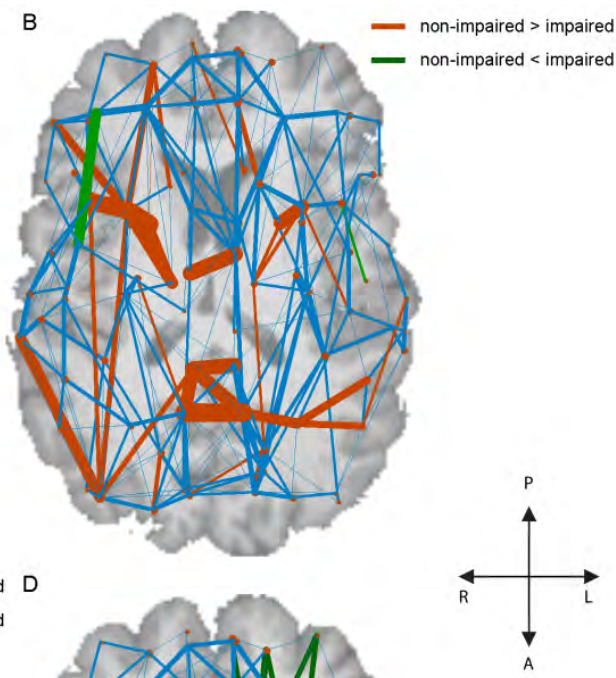

control > non-impaired control < non-impaired

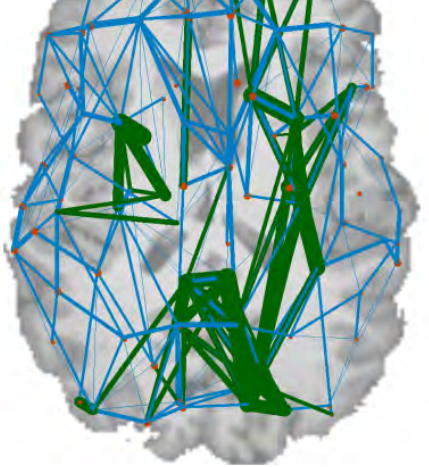

Figure 4.3 Analysis of individual tract volumes. (A) Group differences in tract volume between the healthy control group and the impaired patient group, (B) the non-impaired patient group and the impaired patient group and $(\mathbf{C})$ the healthy control and non-impaired group. Blue-colored lines relate to population average volume weighted structural network. Edge thickness relates to volume of the tract (thick line represents larger tract volume). Red and green edges indicate group differences as indicated in the sub panels. Dots denote the center locations of brain regions. (D) Correlation of FSIQ with tract volume. Edge thickness relates to volume of the tract (thick line represents larger tract volume of connection strength). Thick greencolored edges are significantly correlated with FSIQ in the entire patient group $(p<0.01$, uncorrected). 
A

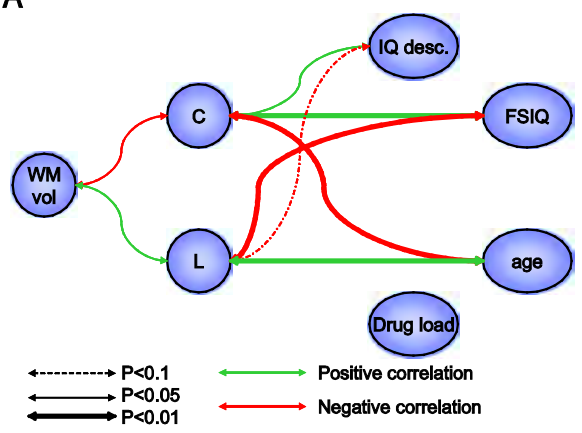

B

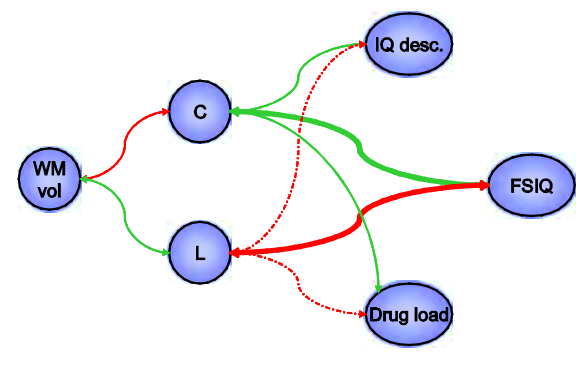

Figure 4.4 Correlation diagrams. (A) Schematic diagram showing the investigated correlations without correction for age and gender in the entire patient group. (B) Partial correlations between variables of interest in the entire patient group with correction for age and gender.

The IQ discrepancy score, was also found to be positively associated with $C(r=0.37$, $p<0.03)$ and a trend for negative association with $L(r=-0.31, p<0.07)$ was found, while correcting for age and gender. A positive IQ discrepancy (no intellectual decline) was associated with a higher $C$.

In the healthy control group, neither FS-IQ nor IQ discrepancy was significantly correlated with $C$ and $L$.

Drug load was negatively associated with $C(r=-0.34, p<0.04)$ and trend for a positive association with $L(r=0.30, p=0.08)$ was found. We also tested for an association between $C$ and $L$ and total number of partial seizures, total number of secondarily generalized seizures and duration of epilepsy, although none of these associations were significant when controlling for age and gender. Table 4.2 and Figure 4.5 display the correlation results.

Table 4.2 Partial correlation table with corrections for age and gender.

\begin{tabular}{lccc}
\hline Network parameter & $K$ & $C$ & $L$ \\
Correlation coefficient $(r)$ & & & \\
\hline FS-IQ & $0.51^{* *}$ & $0.58^{* * *}$ & $-0.57^{* * *}$ \\
IQ discrepancy & $0.35^{* *}$ & $0.37^{* *}$ & $-0.31^{*}$ \\
Drug load & $-0.32^{*}$ & $-0.34^{* *}$ & $0.30^{*}$ \\
SGS count & -0.01 & -0.01 & -0.01 \\
PS count & -0.06 & -0.24 & 0.18 \\
Duration of epilepsy & $-0.32^{*}$ & -0.20 & 0.12 \\
\hline
\end{tabular}

$* * * p<0.001, * * p<0.05, * p<0.1$

$K=$ number of edges, $C=$ weighted cluster coefficient, $L=$ weighted path length, SGS = Secondarily Gen eralized Seizures, PS = Partial seizures. 

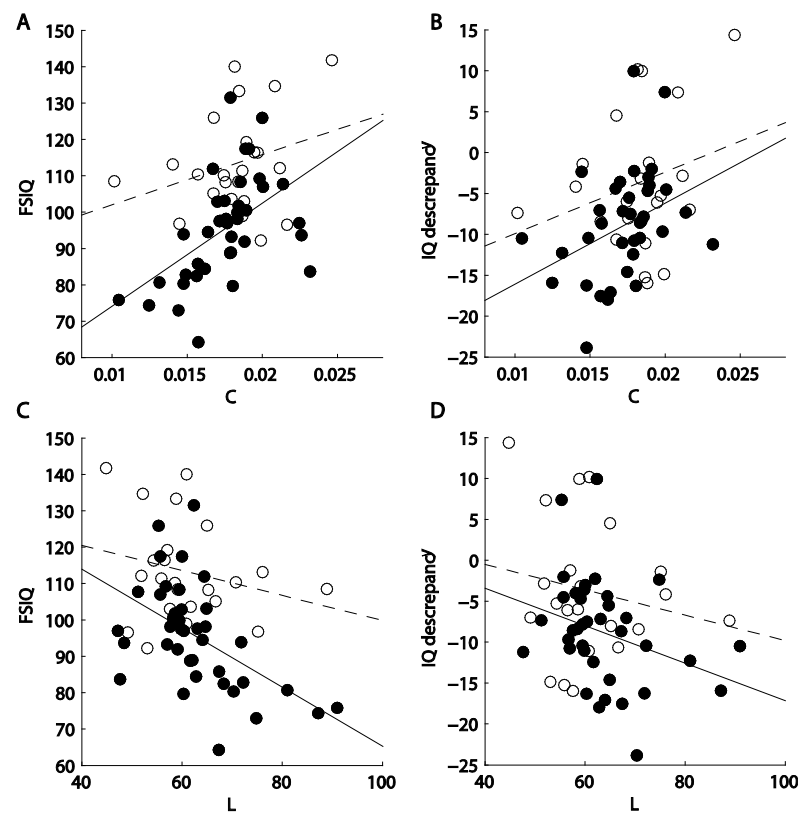

Figure 4.5 Correlation plots. (A) Plot showing correlation between full IQ (FS-IQ) and weighted clustering coefficient (C), for the patient group (black dots, solid regression line) and healthy controls (open dots, dashed regression line). (B) $C$ versus IQ discrepancy. (C) Path length $(L)$ versus FSIQ. (D) $L$ versus IQ discrepancy.

\section{DISCUSSION}

\section{Main findings}

This study was performed to find abnormalities in white matter network organization in patients with cryptogenic localization related epilepsy that may explain the associated cognitive decline in comparison to healthy controls. A number of novel observations were obtained regarding the axonal organization of white matter in relation to cognitive impairment in chronic epilepsy. First, epilepsy patients with cognitive impairment displayed less efficient white matter network properties in the form of a lower weighted clustering and a higher weighted mean path length compared to epilepsy patients without cognitive impairment and healthy controls. No differences in whole brain white matter volumes were noticed. Second, in the entire patient group, a decreased weighted cluster coefficient and increased weighted path length were strongly correlated with lower FS-IQ and stronger IQ discrepancy. Controlling for age and gender did not affect this observation. Third, whole brain white matter in itself was not correlated with FS-IQ or IQ discrepancy, although 
significant correlations between total white matter volume and network parameters were found.

\section{White matter correlates of cognitive impairment}

The weighted clustering coefficient and weighted path length were significantly different in the cognitively impaired patient group compared to both the unimpaired patient and healthy control group. A lower weighted clustering indicates that local brain regions are mutually weaker interconnected and a higher weighted path length refers to a less globally connected brain in the sense that more distal brain regions are less efficiently connected. As the clustering was lower and the path length was higher for cognitively impaired patients the networks can be interpreted as less efficiently organized. The observation that the white matter organization rather than the white matter volume appears to be disrupted is a novel finding in epilepsy. The origin of this finding remains unknown, although different mechanisms could lead to less efficient networks, for instance subtle alterations in tract volumes due to atrophy and transneuronal degeneration (Kodama et al., 2003; Beirowski et al., 2005) or compensatory mechanisms (Schlaug et al., 2009). Additionally, patients with cryptogenic localization-related epilepsies might have a diffuse underlying pathology such as cortical dysplasia type I or microdysgenesis (Sisodiya, 2004). Although undetected at 3T, these are real anatomical disorders, and could also explain the poor connectivity and intellectual impairment as much as the epilepsy itself.

Previously, macro-structural abnormalities in grey and white matter volumes were reported in epilepsy (Seidenberg et al., 2005). For instance, Hermann et al (Hermann et al., 2010) reports abnormal white matter development in children with new onset epilepsy and Focke et al. (Focke et al., 2008a) showed that grey matter volume was associated with cognitive scores in TLE patients with hippocampal sclerosis, although these changes did not reside in narrowly circumscribed brain regions. Local macrostructural WM lesions have been shown not to be associated with cognitive impairment in chronic epilepsy (Jansen et al., 2008). Micro-structural white matter abnormalities have also been found (Focke et al., 2008b; Meng et al., 2010). Additionally, diffusion indices were found to be correlated with various cognitive scores (Riley et al., 2010). The above mentioned studies were focused on finding regions in which indices of local white matter fiber integrity were abnormal. However, they have not investigated axonal connectivity per se. In Powel et al. (Powell et al., 2007) and Yogarajah et al. (Yogarajah et al., 2008) tractography was used to study axonal connectivity more directly. Tract volume and tract FA were used to study specific temporal and frontal lobe white matter tracts. Their results showed reduced volume and FA in the ipsilateral hemisphere (mainly in the left TLE compared with the control group), while an increase was seen in the contralateral hemisphere. In addition, Yogarajah et al. (Yogarajah et al., 2008) found an association between these white matter alterations and decreased memory scores. Both studies provide 
evidence for reorganization of white matter connections in TLE, and support our findings that white matter connectivity alterations might underlie cognitive impairments in patients with cryptogenic localization related epilepsy. However, our study provides a more complete picture of white matter disruption, as altered whole brain network properties were observed as opposed to disruptions in a predefined set of connections.

Cerebral network properties have previously been studied in healthy subjects with fiber tractography (Hagmann et al., 2008; Gong et al., 2009) of which the properties have been linked to gender and brain size (Yan et al., 2010), age and development (Hagmann et al., 2010) and intelligence (Li et al., 2009). Our finding that age, gender, and brain size are related to structural network properties is in agreement with these studies. Furthermore, our results concur with those of Li et al. (Li et al., 2009), who also found a negative correlation between FS-IQ and path length. Li et al. (Li et al., 2009) also found a positive correlation between $C$ and FS-IQ, although this was not significant. These results support the notion that network efficiency and cognitive performance are related (Bosma et al., 2009; Li et al., 2009; van den Heuvel et al., 2009), and could imply that in vivo measurements of brain network efficiency provide a more sensitive marker for cognitive decline at an early stage.

\section{Clinical perspective}

For future cognitive prognosis of patients with epilepsy it would be clinically relevant to know whether patients are prone to developing cognitive impairment. The presented method of DTI combined with graph theoretical network analysis has the potential to discern patients with increased vulnerability for cognitive impairment on the basis of inefficient network parameters (i.e. low clustering and/or high path length). The high reproducibility (Vaessen et al., 2010; Bassett et al., 2011) of the imaging technique in combination with the calculated network parameters could make this imaging method a promising adjunctive tool in the clinical diagnosis of cognitive co-morbidity in epilepsy and may influence clinical therapeutic decision making. DTI does not require any cognitive task performance during scanning and is therefore more applicable in patients with severe cognitive problems.

\section{Methodological considerations}

We used a DTI acquisition with 15 gradient directions at $b=800 \mathrm{~s} / \mathrm{mm}^{2}$. An important question is whether a different acquisition scheme with higher angular resolution and different $b$-values (Hagmann et al., 2010) will influence the results. Recent studies on the effects of different acquisition schemes on graph metrics (Vaessen et al., 2010; Zalesky et al., 2010b) revealed that the effect of angular resolution and $b$-value was small, in healthy volunteers. We expect therefore no strong effects in our study population without macroscopic lesions, but future studies might address the issue of 
how various DWI acquisition schemes influence group differences and effects in a clinical population.

The main emphasis of this study was on connectivity of the cerebral cortex through the white matter. This requires the tracking algorithm to reach parts of the brain close to the grey matter. Therefore, we used probabilistic fiber tracking in combination with a liberal curvature threshold of 60 degrees (calculated over the length of a voxel).

The AAL template defines regions with a variety of different sizes, which may bias certain nodal measurements such as node degree. An additional analysis was performed to check whether correcting connection weights for region size would alter the results. As expected, correction for region size did not have a large effect (see Supplementary data).

An overall lower number of fiber tracts was found in the impaired patient group compared to the healthy control and unimpaired patient group. These results cannot be interpreted unambiguously as the current state of technology does not allow to infer whether a missing fiber tract is actually anatomically non-existing or could not be reconstructed as a result of algorithm or data features. Furthermore, the number of edges in a network is a strong confounder for subsequent network parameters (van Wijk et al., 2010). Therefore, a network was constructed with edges that could be reliably found within every subject. It is likely that relevant pathological information exist in the edges removed from the final network analysis, since the number of edges was lower in the cognitively impaired patient group compared to the unimpaired and healthy control group and this number was associated with FS-IQ in the whole patient group. That, even after such stringent data filtering, we can still observe strong and significant differences in network parameters, shows the robustness of the current approach.

Tract volume was used to weight the edges in the calculation of the connection matrix and consequently the network measures $C$ and $L$. Other indices of tract integrity, such as fractional anisotropy, mean diffusivity, level of myelination and the number of reconstructed fibers have previously been applied (Gong et al., 2009; Li et al., 2009; Hagmann et al., 2010; van den Heuvel et al., 2010). Currently, no consensus prevails which weighting method describes best the fiber tract integrity or is most sensitive to pathological effects. To test the robustness of our results, we also constructed network weighted by fractional anisotropy (FA) and mean diffusivity (MD) values. The results of those networks were comparable with those of the presented volume networks (see Supplementary data).

The statistical tests with graph metrics were not corrected for multiple comparisons, as our two main study objectives were to investigate whether graph metrics would differ between the studied groups and would show a correlation with cognitive scores. Other analyses (such as a relation between graph metrics and epilepsy related variables) were of a more explanatory nature. Future studies with a larger number of subjects and more specific hypothesis might benefit from a proper multiple 
comparisons correction that needs to be tailored specifically to network data (Zalesky et al., 2010a).

\section{CONCLUSIONS}

The application of graph theoretical analysis on whole brain diffusion tensor imaging data enabled the detection of loss of axonal network organization in the white matter in cognitively impaired patients with cryptogenic localization related epilepsy. Here, deviations in network organization appear to be sensitive to cognitive decline even in patients without MRI-visible lesions. More specifically, it is not the total volume of the white matter that has changed, but the network organization of the white matter, in terms of relative volume contributions of multiple white matter fiber bundles, that is affected in cognitively impaired patients with epilepsy. 


\section{REFERENCES}

Ashburner J, Friston KJ (1999) Nonlinear spatial normalization using basis functions. Hum Brain Mapp 7:254266.

Bassett DS, Brown JA, Deshpande V, Carlson JM, Grafton ST (2011) Conserved and variable architecture of human white matter connectivity. Neuroimage 54:1262-1279.

Beirowski B, Adalbert R, Wagner D, Grumme DS, Addicks K, Ribchester RR, Coleman MP (2005) The progressive nature of Wallerian degeneration in wild-type and slow Wallerian degeneration (WIdS) nerves. BMC Neurosci 6:6.

Benjamini Y, Hochberg Y (1995) Controlling the false discovery rate - a practical and powerful approach to multiple testing. Journal of the Royal Statistical Society Series B-Methodological 57:289-300.

Bonelli SB, Powell RH, Yogarajah M, Samson RS, Symms MR, Thompson PJ, Koepp MJ, Duncan JS (2010) Imaging memory in temporal lobe epilepsy: predicting the effects of temporal lobe resection. Brain 133:1186-1199.

Bosma I, Reijneveld JC, Klein M, Douw L, van Dijk BW, Heimans JJ, Stam CJ (2009) Disturbed functional brain networks and neurocognitive function in low-grade glioma patients: a graph theoretical analysis of resting-state MEG. Nonlinear Biomed Phys 3:9.

Bressler SL, Menon V (2010) Large-scale brain networks in cognition: emerging methods and principles. Trends Cogn Sci 14:277-290.

Bullmore E, Sporns O (2009) Complex brain networks: graph theoretical analysis of structural and functional systems. Nat Rev Neurosci 10:186-198.

Cook PA, Bai Y, Nedjati-Gilani S, Seunarine KK, Hall MG, Parker GJ, Alexander DC (2006) Camino: OpenSource Diffusion-MRI Reconstruction and Processing. In: 14th Scientific Meeting of the International Society for Magnetic Resonance in Medicine, p 2759. Seatle (WA): 14th ISMRM.

Crofts JJ, Higham DJ, Bosnell R, Jbabdi S, Matthews PM, Behrens TE, Johansen-Berg H (2011) Network analysis detects changes in the contralesional hemisphere following stroke. Neuroimage 54:161169.

Deary IJ, Penke L, Johnson W (2010) The neuroscience of human intelligence differences. Nat Rev Neurosci 11:201-211.

Diehl B, Busch RM, Duncan JS, Piao Z, Tkach J, Luders HO (2008) Abnormalities in diffusion tensor imaging of the uncinate fasciculus relate to reduced memory in temporal lobe epilepsy. Epilepsia 49:14091418.

Elger CE, Hel mstaedter C, Kurthen M (2004) Chronic epilepsy and cognition. Lancet Neurol 3:663-672.

Farrell JA, Landman BA, Jones CK, Smith SA, Prince JL, van ZijI PC, Mori S (2007) Effects of signal-to-noise ratio on the accuracy and reproducibility of diffusion tensor imaging-derived fractional anisotropy, mean diffusivity, and principal eigenvector measurements at $1.5 \mathrm{~T}$. J Magn Reson Imaging 26:756-767.

Focke NK, Thompson PJ, Duncan JS (2008a) Correlation of cognitive functions with voxel-based morphometry in patients with hippocampal sclerosis. Epilepsy Behav 12:472-476.

Focke NK, Yogarajah M, Bonelli SB, Bartlett PA, Symms MR, Duncan JS (2008b) Voxel-based diffusion tensor imaging in patients with mesial temporal lobe epilepsy and hippocampal sclerosis. Neuroimage 40:728-737.

Glasser MF, Rilling JK (2008) DTI tractography of the human brain's language pathways. Cereb Cortex 18:2471-2482. 
Gong G, He Y, Concha L, Lebel C, Gross DW, Evans AC, Beaulieu C (2009) Mapping anatomical connectivity patterns of human cerebral cortex using in vivo diffusion tensor imaging tractography. Cereb Cortex 19:524-536.

Hagmann P, Cammoun L, Gigandet X, Meuli R, Honey CJ, Wedeen VJ, Sporns O (2008) Mapping the structural core of human cerebral cortex. PLoS Biol 6:e159.

Hagmann P, Sporns O, Madan N, Cammoun L, Pienaar R, Wedeen VJ, Meuli R, Thiran JP, Grant PE (2010) White matter maturation reshapes structural connectivity in the late developing human brain. Proceedings of the National Academy of Sciences.

Helmstaedter C, Kockelmann E (2006) Cognitive outcomes in patients with chronic temporal lobe epilepsy. Epilepsia 47 Suppl 2:96-98.

Hermann BP, Gold J, Pusakulich R, Wyler AR, Randolph C, Rankin G, Hoy W (1995) Wechsler adult intelligence scale-revised in the evaluation of anterior temporal lobectomy candidates. Epilepsia 36:480-487.

Hermann BP, Dabbs K, Becker T, Jones JE, Myers YGA, Wendt G, Koehn MA, Sheth R, Seidenberg M (2010) Brain development in children with new onset epilepsy: A prospective controlled cohort investigation. Epilepsia 51:2038-2046.

Jansen JF, Vlooswijk MC, Majoie HM, de Krom MC, Aldenkamp AP, Hofman PA, Backes WH (2008) White matter lesions in patients with localization-related epilepsy. Invest Radiol 43:552-558.

Jeukens CR, Vlooswijk MC, Majoie HJ, de Krom MC, Aldenkamp AP, Hofman PA, Jansen JF, Backes WH (2009) Hippocampal MRI volumetry at 3 Tesla: reliability and practical guidance. Invest Radiol 44:509-517.

Kodama F, Ogawa T, Sugihara S, Kamba M, Kohaya N, Kondo S, Kinoshita T (2003) Transneuronal degeneration in patients with temporal lobe epilepsy: evaluation by MR imaging. Eur Radiol 13:2180-2185

Li Y, Liu Y, Li J, Qin W, Li K, Yu C, Jiang T (2009) Brain anatomical network and intelligence. PLoS Comput Biol 5:e1000395.

McDonald CR, Ahmadi ME, Hagler DJ, Tecoma ES, Iragui VJ, Gharapetian L, Dale AM, Halgren E (2008) Diffusion tensor imaging correlates of memory and language impairments in temporal lobe epilepsy. Neurology 71:1869-1876.

Meador KJ, Hermann B (2010) How localized is localization-related epilepsy? Neurology 75:386-387.

Meng L, Xiang J, Kotecha R, Rose D, Zhao H, Zhao D, Yang J, Degrauw T (2010) White matter abnormalities in children and adolescents with temporal lobe epilepsy. Magn Reson Imaging 28:1290-1298.

Onnela J-P, Saram, auml, ki J, Kert, eacute, sz J, aacute, nos, Kaski K (2005) Intensity and coherence of motifs in weighted complex networks. Physical Review E 71:065103.

Oyegbile TO, Dow C, Jones J, Bell B, Rutecki P, Sheth R, Seidenberg M, Hermann BP (2004) The nature and course of neuropsychological morbidity in chronic temporal lobe epilepsy. Neurology 62:17361742.

Parker GJ, Haroon HA, Wheeler-Kingshott CA (2003) A framework for a streamline-based probabilistic index of connectivity (PICo) using a structural interpretation of MRI diffusion measurements. Journal of Magnetic Resonance Imaging 18:242-254.

Powell HW, Parker GJ, Alexander DC, Symms MR, Boulby PA, Wheeler-Kingshott CA, Barker GJ, Koepp MJ, Duncan JS (2007) Abnormalities of language networks in temporal lobe epilepsy. Neuroimage 36:209-221.

Riley JD, Franklin DL, Choi V, Kim RC, Binder DK, Cramer SC, Lin JJ (2010) Altered white matter integrity in temporal lobe epilepsy: association with cognitive and clinical profiles. Epilepsia 51:536-545. 
Rubinov M, Sporns O (2009) Complex network measures of brain connectivity: Uses and interpretations. Neuroimage.

Rubinov M, Sporns O (2010) Complex network measures of brain connectivity: uses and interpretations. Neuroimage 52:1059-1069.

Schlaug G, Marchina S, Norton A (2009) Evidence for plasticity in white-matter tracts of patients with chronic Broca's aphasia undergoing intense intonation-based speech therapy. Ann N Y Acad Sci 1169:385-394.

Schoenberg MR, Scott JG, Duff K, Adams RL (2002) Estimation of WAIS-III intelligence from combined performance and demographic variables: development of the OPIE-3. Clin Neuropsychol 16:426437.

Seidenberg M, Geary E, Hermann B (2005) Investigating temporal lobe contribution to confrontation naming using MRI quantitative volumetrics. J Int Neuropsychol Soc 11:358-366.

Sisodiya SM (2004) Malformations of cortical development: burdens and insights from important causes of human epilepsy. Lancet Neurol 3:29-38.

Smith SM (2002) Fast robust automated brain extraction. Human Brain Mapping 17:143-155.

Smith SM, Jenkinson M, Wool rich MW, Beckmann CF, Behrens TE, Johansen-Berg H, Bannister PR, De Luca M, Drobnjak I, Flitney DE, Niazy RK, Saunders J, Vickers J, Zhang Y, De Stefano N, Brady JM, Matthews PM (2004) Advances in functional and structural MR image analysis and implementation as FSL. Neuroimage 23 Suppl 1:S208-219.

Stam CJ, Reijneveld JC (2007) Graph theoretical analysis of complex networks in the brain. Nonlinear Biomed Phys 1:3.

Thomas C, Moya L, Avidan G, Humphreys K, Jung KJ, Peterson MA, Behrmann M (2008) Reduction in white matter connectivity, revealed by diffusion tensor imaging, may account for age-related changes in face perception. J Cogn Neurosci 20:268-284.

Toosy AT, Ciccarelli O, Parker GJ, Wheeler-Kingshott CA, Miller DH, Thompson AJ (2004) Characterizing function-structure relationships in the human visual system with functional MRI and diffusion tensor imaging. Neuroimage 21:1452-1463.

Tzourio-Mazoyer N, Landeau B, Papathanassiou D, Crivello F, Etard O, Delcroix N, Mazoyer B, Joliot M (2002) Automated Anatomical Labeling of Activations in SPM Using a Macroscopic Anatomical Parcellation of the MNI MRI Single-Subject Brain. Neuroimage 15:273-289.

Vaessen MJ, Hofman PA, Tijssen HN, Aldenkamp AP, Jansen JF, Backes WH (2010) The effect and reproducibility of different clinical DTI gradient sets on small world brain connectivity measures. Neuroimage 51:1106-1116.

van den Heuvel MP, Stam CJ, Kahn RS, Hulshoff Pol HE (2009) Efficiency of functional brain networks and intellectual performance. J Neurosci 29:7619-7624.

van den Heuvel MP, Mandl RC, Stam CJ, Kahn RS, Hulshoff Pol HE (2010) Aberrant frontal and temporal complex network structure in schizophrenia: a graph theoretical analysis. J Neurosci 30:1591515926.

van Wijk BCM, Stam CJ, Daffertshofer A (2010) Comparing Brain Networks of Different Size and Connectivity Density Using Graph Theory. PLoS One $5:$ e13701.

Vlooswijk MC, Jansen JF, Majoie HJ, Hofman PA, de Krom MC, Aldenkamp AP, Backes WH (2010) Functional connectivity and language impairment in cryptogenic localization-related epilepsy. Neurology 75:395-402.

Wechsler D (1997) Manual for the Wechsler Adult Intelligence Scale-III. San Antonio: The Psychological Corporation. 
Yan C, Gong G, Wang J, Wang D, Liu D, Zhu C, Chen ZJ, Evans A, Zang Y, He Y (2010) Sex- and Brain SizeRelated Small-World Structural Cortical Networks in Young Adults: A DTI Tractography Study. Cereb Cortex.

Yogarajah M, Powell HW, Parker GJ, Alexander DC, Thompson PJ, Symms MR, Boulby P, Wheeler-Kingshott CA, Barker GJ, Koepp MJ, Duncan JS (2008) Tractography of the parahippocampal gyrus and material specific memory impairment in unilateral temporal lobe epilepsy. Neuroimage 40:17551764.

Zalesky A, Fornito A, Bullmore ET (2010a) Network-based statistic: identifying differences in brain networks. Neuroimage 53:1197-1207.

Zalesky A, Fornito A, Harding IH, Cocchi L, Yucel M, Pantel is C, Bullmore ET (2010b) Whole-brain anatomical networks: does the choice of nodes matter? Neuroimage 50:970-983.

Zalesky A, Fornito A, Seal ML, Cocchi L, Westin CF, Bullmore ET, Egan GF, Pantelis C (2010c) Disrupted Axonal Fiber Connectivity in Schizophrenia. Biol Psychiatry.

Zhang Y, Brady M, Smith S (2001) Segmentation of brain MR images through a hidden Markov random field model and the expectation-maximization algorithm. IEEE transactions on medical imaging 20:4557. 


\section{SUPPLEMENTARY DATA}

\section{FA, MD and stream count weighted networks}

Tract volume was used to weight the edges in the calculation of the connection matrix and consequently the network measures $C$ and $L$. White matter fiber tract volumes derived from tractography have previously been used in several contexts. In (Powell et al., 2007) and (Yogarajah et al., 2008) tract volume and tract FA are used to study specific temporal and frontal lobe white matter tracts. Their results show reduced volume and FA in the ipsi-lateral hemisphere (mainly in the left TLE compared with the control group), while the opposite was seen in the contra-lateral hemisphere. In other domains than epilepsy, tract volume has also been used to study white matter properties. In (Glasser and Rilling, 2008), tract volume was used to study asymmetry of language pathways. In (Thomas et al., 2008), tract volume was used to study the relationship between age and a decline in face perception. Together, these studies indicate that tract volume indeed is a sensitive measure to study variations in white matter connectivity for a broad range of topics.

Other indices of tract integrity, such as fractional anisotropy, mean diffusivity, level of myelination and the number of reconstructed fibers have previously been applied (Gong et al., 2009; Li et al., 2009; Hagmann et al., 2010; van den Heuvel et al., 2010). Currently no consensus prevails which weighting method describes best the fiber tract integrity or is most sensitive to pathological effects.

To test the robustness of our results, we also constructed network weighted by fractional anisotropy (FA), mean diffusivity (MD) values and number of reconstructed fibers. The mean of the FA or $1 / \mathrm{MD}$ voxel values of a tract were used as weight. For the number of reconstructed fibers weighting, the number of streamlines connection two regions was counted (streamline count, SC). Connectivity matrices were divided by the mean weight of all connections prior to network parameters calculation.

Results of correlation and partial correlation with FSIQ, etc are shown in Table S.4.1.

Figure S.4.1 Results of the different weighting schemes.

\begin{tabular}{|c|c|c|c|c|c|c|c|}
\hline & & LFA & CFA & LMD & CMD & LSC & CSC \\
\hline $\begin{array}{l}\text { Partial correlation } \\
\text { correcting for age \& } \\
\text { gender }\end{array}$ & FS-IQ & -0.25 & $0.35^{* *}$ & -0.25 & $0.31 *$ & -0.06 & $0.39 * *$ \\
\hline Pearson's correlation & FS-IQ & $-0.27 *$ & $0.36 * *$ & $-0.27^{*}$ & $0.35^{* *}$ & 0.25 & 0.07 \\
\hline
\end{tabular}

${ }^{* *} p<0.05, * p<=0.1$

LFA = weighted path length for the FA weighted network, CFA = weighted cluster coefficient for the FA weighted network. The same convention holds for LMD, CMD, LSC, CSC. SC = streamline count. 
As can be seen from Table S.4.1, the results of the FA, MD and SC weighted networks were comparable with those of the presented volume networks. Lower FA values and lower $1 / \mathrm{MD}$ values are normally associated with impaired white matter integrity. For tract volume, one can hypothesize that a reduced tract volume is related to some sort of WM damage. It is important to note that FA or MD values are not necessarily correlated with tract volume. A large tract might traverse an area within the deep with matter (with relatively high FA). Similarly, a small tract might traverse closer to the GM (usually with lower FA values, for instance u-shaped fibers), but just as well traverse the deep white matter (relatively high FA, for instance the optic radiation). Still, changes between subjects should have the same direction (e.g. lower FA, lower \# of fibers and lower volume in a damaged tract). Therefore, we would expect network changes to also show the same effect between the different weighting schemes, which is precisely what we observed.

\section{Correction for VOI size}

The AAL template defines regions with very different sizes, which may bias certain nodal measurements such as node degree. An additional analysis was performed to check whether correcting connection weights for region size would alter the results.

Table S.4.2 displays the differences in partial correlation with FSIQ for the connectivity matrices with and without correction for VOI size (the volume of each tract was divided by ICV and by the mean volume of the VOI's connected by the tract). As expected, correction for region size did not have a large effect.

Figure S.4.2 Results of the different weighting schemes. correlation of $C$ and $L$ with FS-IQ of the volume weighted networks, with and without correction for region size.

\begin{tabular}{lllll}
\hline $\begin{array}{l}\text { Cvolume weighted } \\
\text { network }\end{array}$ & $\begin{array}{l}\text { L volume weighted } \\
\text { network }\end{array}$ & $\begin{array}{l}\text { C volume weighted } \\
\text { network, corrected for } \\
\text { region size }\end{array}$ & $\begin{array}{l}\text { L volume weighted } \\
\text { network, corrected } \\
\text { for region size }\end{array}$ \\
\hline FS-IQ & $r=0.58, p<0.001$ & $r=-0.57, p<0.001$ & $r=0.44, p<0.007$ & $r=-0.44, p<0.007$ \\
\hline
\end{tabular}




\section{CHAPTER 5}

\section{Abnormal modular organization of functional networks in cognitively impaired children with}

frontal lobe epilepsy

M.J. Vaessen, H.M.H. Braakman, J.S. Heerink, J.F.A. Jansen, M.H.J.A. Debeij-van Hall, P.A.M. Hofman, A.P. Aldenkamp, W.H. Backes 


\section{ABSTRACT}

Many children with frontal lobe epilepsy (FLE) have significant cognitive co-morbidity, for which the underlying mechanism has not yet been unravelled, but is likely related to disturbed cerebral network integrity. Using resting-state fMRI we investigated whether cerebral network characteristics are associated with epilepsy and cognitive co-morbidity.

We included 37 children with FLE and 41 healthy age-matched controls. Cognitive performance was determined by means of a computerized visual searching task (CVST). A connectivity matrix for 82 cortical and subcortical brain regions was generated for each subject by calculating the inter-regional correlation of the fMRI time-signals. From the connectivity matrix graph metrics were calculated and the anatomical configuration of aberrant connections and modular organisation was investigated.

Both patients and controls displayed efficiently organized networks. However, FLE patients displayed a higher modularity, implying that sub-networks are less interconnected. Impaired cognition was associated with higher modularity scores and abnormal modular organization of the brain, which was mainly expressed as a decrease of long-range and an increase of inter-hemispheric connectivity in patients.We show that network modularity analysis provides a sensitive marker for cognitive impairment in FLE and suggest that abnormally interconnected functional sub-networks of the brain might underlie the cognitive problems in children with FLE. 


\section{INTRODUCTION}

Frontal lobe epilepsy (FLE) is considered the second most common type of the localization-related (partial) epilepsies of childhood, after temporal lobe epilepsy, and accounts for $20-30 \%$ of partial epilepsies (Manford et al., 1992). Pediatric FLE is frequently complicated by cognitive impairment and behavioral disturbances. FLE impacts a broad scale of cognitive domains, broader than the typical frontal functions (Centeno et al., 2010; Braakman et al., 2011). In children with FLE, the learning difficulties may even precede seizure onset, suggesting a nontrivial relation between seizures and cognitive problems, which has not been unraveled thus far (Prevost et al., 2006; Patrikelis et al., 2009).

The broad scale of affected cognitive domains hints at a network disturbance, rather than disturbance of localized processes. In line with this suggestion, conventional structural MRI studies have not shown an anatomical substrate for the neuronal mechanisms leading to cognitive impairment in FLE (Harvey et al., 1993; Laskowitz et al., 1995; Lorenzo et al., 1995; Provini et al., 1999; Lawson et al., 2002). To resolve this, functional MRI techniques may prove novel and valuable insights. Resting-state $\mathrm{fMRI}(\mathrm{RS}-\mathrm{fMRI})$ is a functional imaging technique that may be useful in understanding the neuronal mechanisms behind cognitive co-morbidity in neurological disorders (Fox and Raichle, 2007). RS-fMRI enables the investigation of the intrinsic functional organization of the brain, in contrast to the cerebral effect of tasks executed by subjects. This intrinsic functional organization is called "functional connectivity", which is defined by the temporal correlation of neuronal activity-induced patterns of anatomically different brain regions (Friston, 1994; Van den Heuvel and Hulshoff Pol, 2010). Previous studies have demonstrated disturbances in functional connectivity networks in the brains of adult epilepsy patients (Waites et al., 2006; Bettus et al., 2009; Liao et al., 2010; Pereira et al., 2010; Vlooswijk et al., 2010; Pravata et al., 2011; Vlooswijk et al., 2011). These studies focused on local connectivity abnormalities, i.e. only a few regions of the brain were analysed or considered as a reference. Given the heterogeneous expression of cognitive deficits in FLE (Braakman et al., 2011), it is advantageous to investigate the whole brain network. In mathematical terms, the brain can be modeled as a system consisting of nodes (brain regions) and edges (connections) between them. The strength of a connection is then quantified by the degree of correlation of the dynamic fluctuations between a pair of nodes, i.e. brain regions. An elegant way to understand and quantify the organization of this system of nodes and edges is to calculate graph theoretical metrics of the whole brain network (Rubinov and Sporns, 2010). Metrics that provide information on the amount of integration and segregation over the entire brain are path length and clustering. In addition to whole brain network organization, graph theoretical metrics can also describe the interconnection of sub-networks in the whole brain network by modularity analysis (Newman, 2006), see Table 5.1 for a detailed description. The modular structure of the brain network is thought to be important for cognitive 
abilities as increases in coherent activity between functional systems might facilitate information integration (van den Heuvel et al., 2009) and adaptive behavior (Power et al., 2010).

Based on the hypotheses that children with FLE have an abnormal brain organization caused by interference of epilepsy with normal development and that this organization may affect cognitive function, we have analyzed cerebral functional connectivity of children with FLE in correlation to their cognitive performance using graph theoretical network parameters.

Table 5.1 Partial correlation table with corrections for age and gender.

\begin{tabular}{ll}
\hline Measure & Interpretation \\
\hline Binary characteristic path length & $\begin{array}{l}\text { Characteristic path length is defined as the average } \\
\text { geodesic distance, in number of edges, connecting any two } \\
\text { nodes in the graph, where } d_{i, j} \text { is the length of the shortest } \\
\text { path between nodes } i \text { and } j . \text { The characteristic path length } \\
\text { is a measure of how well connected a network is. Small } \\
\text { characteristic path length indicates an average short } \\
\text { distance between any two nodes, i.e. they can be reached } \\
\text { through a small number of steps. }\end{array}$
\end{tabular}

Weighted characteristic path length

$$
L_{w}=\frac{1}{N(N-1)} \sum_{i, j \in G, i \neq j} w_{i, j}
$$

Binary clustering coefficient

$$
C_{b}=\frac{1}{N} \sum_{i} \frac{\sum_{j, m}\left(A_{i, j} A_{j, m} A_{m, i}\right)}{k_{i}\left(k_{i}-1\right)}
$$

Weighted clustering coefficient

$$
C_{w}=\frac{1}{N} \sum_{i} \frac{\sum_{j, m}\left(w_{i, j} w_{j, m} w_{m, i}\right)^{1 / 3}}{k_{i}\left(k_{i}-1\right)}
$$

Modularity

$$
Q=\frac{1}{2 K} \sum_{i, j}\left[A_{i, j}-\frac{k_{i} k_{j}}{2 K}\right] \delta\left(m_{i}, m_{j}\right)
$$

The weighted characteristic path length is defined as the average of the shortest paths connecting any two nodes in the graph, where $w_{i, j}$ is the sum of weights of the shortest weighted path between nodes $i$ and $j$. The characteristic path length is a measure of how well connected a network is. In the case of a weighted network higher connectivity strength will decrease the distance between two nodes, and thus a short path length indicates that, on average, any two nodes are connected by one or several strong connections.

The cluster coefficient is defined as the number of edges connecting the neighbours of a node divided by the maximum number of edges possible between neighbouring nodes. The cluster coefficient of a network is a measure of how many local clusters exist in the network. A high cluster coefficient indicates that the neighbours of a node are often also directly connected to each other, i.e. they form a cluster.

For a weighted graph, the cluster coefficient is high when the direct neighbors of a node are also interconnected and have relatively high connectivity strengths.

The modularity score $Q$ quantifies the degree to which a network can be divided in non-overlapping groups. The membership of node $i$ with a module is $m_{i}$. Hence, $\delta\left(m_{i}, m_{j}\right)=1$ when two nodes $i$ and $j$ are in the same module and 0 otherwise. 


\section{METHODS}

\section{Participants}

Patients with FLE were selected from our reference clinic database and were actively contacted. Inclusion criteria for the patients were: a confirmed cryptogenic (i.e., presumed to be symptomatic, but with unknown aetiology) localization-related epilepsy with an epileptic focus in the frontal lobe, aged between 8 and 13 years, no other disease that could cause cognitive impairment, and no history of brain injury. Healthy age-matched controls were recruited by advertisements in local newspapers. Inclusion criteria were no history of brain injury or cognitive problems and visiting regular education. All subjects and parents gave written informed consent and approval for the study by the local Medical Ethical Committee was obtained.

\section{Neuropsychological assessment}

Cognitive performance was measured using a computerized visual searching task (CVST) (Aldenkamp et al., 2004). This task consists of finding a grid pattern out of 24 patterns which matches the one in the centre of the screen. Grid patterns are displayed in a checkerboard fashion and are numbered from 1 to 24 . The target pattern is marked by an arrow on the right side and is selected by typing the correct number on the keyboard. Twenty different target patterns are presented. After 12 presentations the surrounding grids change. The testee is asked to respond as fast as possible. Results show accuracy and speed of responses and are evaluated within the context of visual (complex) information processing and perceptual mental strategies. The most important variable indicating efficient information processing is the average reaction time.

By determining the average reaction time and the errors being made during the task, an age-corrected cognitive performance score was generated (decile score). After grouping these scores into numbers from 1 (worst score) to 10 (best score), the 3 worst performance scores (1, 2 and 3 ) were considered a manifestation of impaired cognitive performance, while other scores were considered normal or good.

\section{Image acquisition}

MRI was performed on a 3.0-Tesla unit equipped with an 8-channel head coil (Philips Achieva, Philips Medical Systems, Best, The Netherlands). Functional MRI data were acquired using a whole-brain single-shot multi-slice echo-planar imaging (EPI) sequence sensitive to the blood oxygen level-dependent (BOLD) effect, with TR $2 \mathrm{~s}$, TE $35 \mathrm{~ms}$, flip angle $90^{\circ}$, pixel size $2 \times 2 \mathrm{~mm}^{2}, 32$ contiguous 4-mm thick slices per volume, 195 volumes per acquisition, and an acceleration factor (SENSE) of 1.5. For anatomic reference, a T1-weigthed 3D fast field echo was acquired with the following 
parameters: repetition time (TR) $8.1 \mathrm{~ms}$, echo time (TE) $3.7 \mathrm{~ms}$, flip angle $8^{\circ}$, field of view (FOV) $256 \times 256 \times 180 \mathrm{~mm}^{3}$ and voxel size $1 \times 1 \times 1 \mathrm{~mm}^{3}$.

\section{Network construction}

As displayed in Figure 5.1, data analysis sequentially consisted of the following procedures:

(i) preprocessing of the measured $\mathrm{fMRI}$ time series; (ii) anatomical parcellation and connectivity matrix; (iii) network analysis; and (iv) statistical analysis.

\section{Preprocessing of time-series data}

The BOLD images were corrected for motion artifacts using SPM5 (Wellcome Trust Centre for Neuroimaging, UCL, London, UK) software. The images were then high-pass filtered with a $\sigma$ of 25 scans ( 50 seconds) and spatially smoothed $(\sigma=1.7 \mathrm{~mm}$ ) using FSL 4.1.7 (Oxford University, Oxford, UK) software. Subsequently, the CSF and whole brain signal time course were removed from the images using standard linear regression. The resulting residual time-series of the cerebrum were used for further analysis. Lastly, the images were low-pass filtered ( $\sigma=2$ s, i.e. 1 dynamic scan interval) to remove high-frequency noise components. To assess possible confounding of motion parameters, these were compared between the groups.

\section{Anatomical parcellation and connectivity matrix}

Freesurfer (Martinos Center of Biomedical Imaging, Boston, US) software was used to segment the T1 images of each subject into 82 cortical and subcortical regions. Freesurfer uses a surface based alignment procedure, which might be more accurate than a volume based alignment of a cortical atlas (Ghosh et al., 2010).

Using Matlab 7.6.0 (The MathWorks Inc., Natick, US), Pearson's linear correlation coefficients between the region-averaged time-series of all pairs of Freesurfer regions were computed. In this way, for each subject an $82 \times 82$ connectivity matrix was determined. This connectivity matrix included both negative and positive correlation values. The removal of the whole brain average time-series signal tends to shift the correlation distribution to a mean value that is closer to zero, thereby creating negative correlations even if no such correlations are initially present in the data (Van Dijk et al., 2010). Only positive correlations were used for further analysis. Additionally, low (absolute) correlation coefficients could adversely affect the results as they may either represent physiologically relevant signal fluctuations or just noise. To overcome this issue, only a pre-specified number of connections with highest correlation coefficients were selected and all other connections were set to zero (Vaessen et al., 2010). Conceptually, this thresholding procedure can be expressed as a sparsity value relating the connections maintained in the network to the total number of connections possible (Achard and Bullmore, 2007; Vlooswijk et al., 2011). 
In the remainder of this article, results will either be presented for a particular sparsity value or as a function of sparsity.
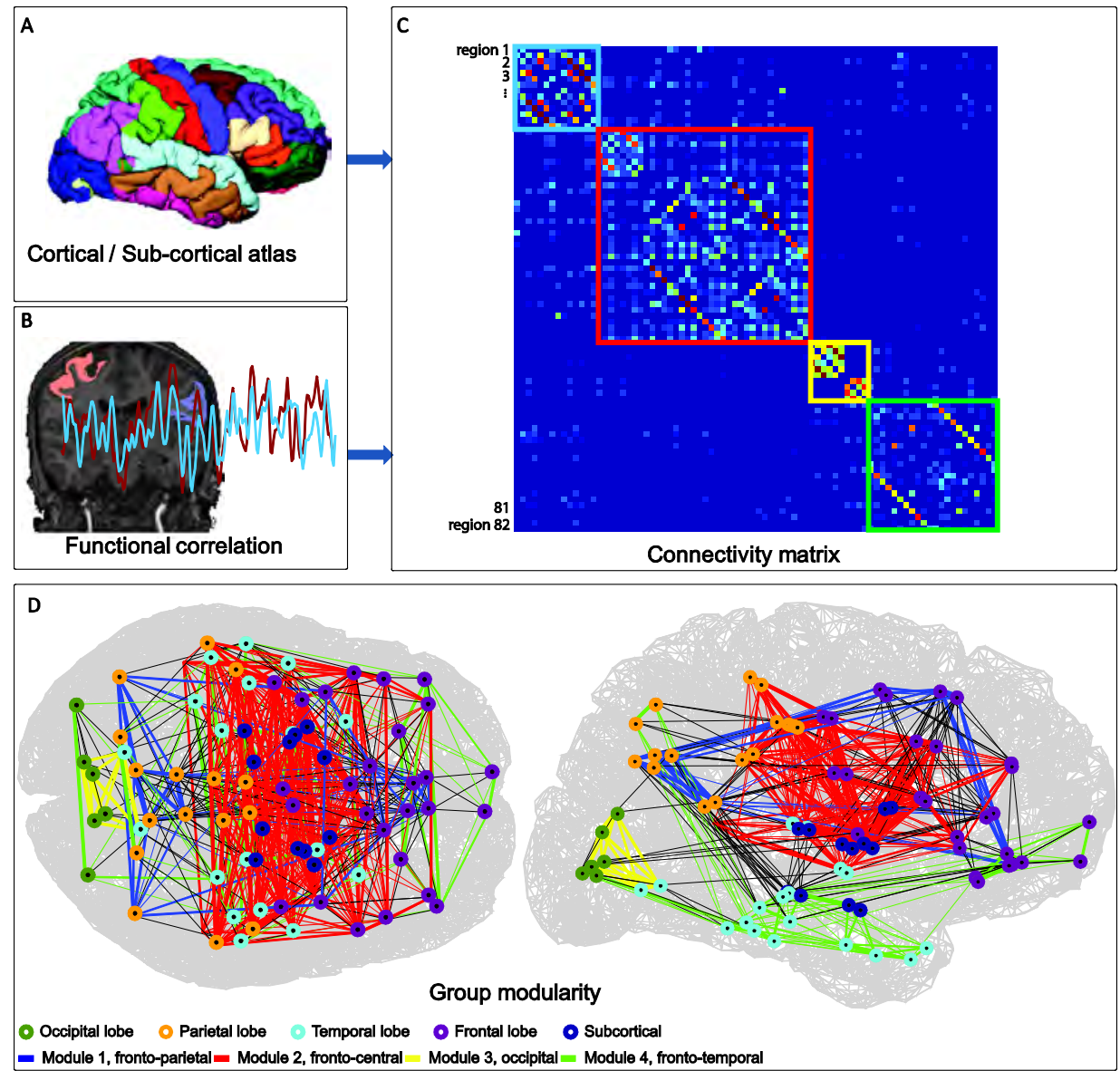

Figure 5.1 (A) T1-image based subject specific parcellation of the cortex and subcortical structures. (B) Mean time signals of the 82 parcellated regions are extracted from the regional signal fluctuations of RS-fMRI. (C) Correlation analysis is performed on each pair of time signals to construct a connectivity matrix for each subject. The colored squares indicate in which module the regions (nodes) and connections reside. (D) Modular organization of the resting state network in the control group. In this figure, the anatomical locations of the regions are indicated as black dots. The surrounding colored circles indicate in which lobe these regions reside. The color of the lines between regions display to what modules the connections belong. It is evident that some modules occupy several lobes, while other are mainly present within one lobe (the yellow occipital module for instance). 


\section{Network analysis}

\section{Network characteristics}

For each subject, values of network metrics were calculated from the individual connectivity matrix. We included 3 widely-used network metrics: characteristic path length, clustering coefficient and modularity, using algorithms implemented in the Brain Connectivity Toolbox (Rubinov and Sporns, 2010). A description of these parameters can be found in Table 5.1 and in Rubinov et al. (Rubinov and Sporns, 2010). For these metrics both the binary and weighted variants were calculated. For the binary metrics the sparsity thresholded connectivity matrix was binarized by setting all edges with a correlation coefficient $>0$ to the value of 1 . For the weighted networks, the connection matrices were divided by the mean connection weight (mean correlation coefficient over all connections), which was also evaluated separately, as this can potentially influence weighted network metrics (Ginestet et al., 2011). For the binary networks equivalent random networks were generated (Maslov and Sneppen, 2002; Vaessen et al., 2010). Clustering and path length from the random networks were compared to the measured networks to assess small-worldness (Watts and Strogatz, 1998).

We compared the entire patient group with controls, the cognitively impaired patients with controls and the cognitively normal patients with the impaired patients. Between-group effects in network parameters were assessed by a two-sample (twotailed) $t$-test.

\section{Correlation between network metrics and cognitive performance}

Pearson's (linear) correlation coefficients were calculated between cognitive performance (CVST reaction time, age and decile scores) and network parameters. We performed this analysis for the entire subject population, as well as for the patient and control groups separately.

\section{Group modularity}

Modularity quantifies the degree to which a brain network is organized in isolated sub-networks. The more isolated the sub-networks are, the higher the modularity. We used an algorithm developed by Newman et al. (Newman, 2006) to visualize the modular structure of the brain. With this algorithm the brain was automatically subdivided into a number of modules (i.e. groups of nodes) with maximal correlation within and minimal correlation between the modules, creating a so-called "optimal community structure" (OCS) of the cerebrum. It is difficult to assess OCS at the group level, because the resulting number and spatial locations of the modules varies between subjects. For group level analysis, a group connectivity matrix was obtained by averaging all individual correlation matrices from a subgroup of subjects (Fair et al., 2009). Another option is to concatenate the time series from all subjects, as is often 
done in fMRI studies using group ICA (Filippini et al., 2009), and to subsequently calculate group connectivity matrices and modularity. Both options were performed, however interpretations of the results were similar. Therefore, only the results from the averaged connectivity matrices are reported here. The OCS for the group matrix was calculated and visualized for (i) the control group, (ii) the entire patient group and (iii) the group of cognitively impaired patients.

\section{Analysis of individual connections}

The individual elements of the connectivity matrices were tested for group differences. Differences between the entire patient group and the control group as well as differences between the cognitively impaired patient group and the control group were assessed by mass uni-variate (2-sided) t-tests. Due to the exploratory nature of this analysis, no stringent multiple comparisons methods were applied. Instead, a liberal significance threshold of $p<0.05$ was used to assess possible group differences.

Furthermore, we investigated whether any aberrant connections would show a particular relation with the modularity analysis or would reveal an effect with connection length. The relation with modularity was investigated by analyzing whether differences would manifest as inter- or intra-modular connections. A possible difference in anatomical orientation was investigated by measuring the angle relative to a strict left-right orientation of connections. Hence, we quantified whether aberrant edges would be oriented in an anterior-posterior of left-right orientation, disregarding their inferior-superior orientation. A possible effect of connection length was assessed by the differences in Euclidian distance between connections that showed either an increase or decrease of connection strength in the entire patient group.

\section{RESULTS}

\section{Preprocessing results}

The motion correction procedure was able to adequately correct for movement in the majority of the cases. The control and patient group did not differ in the amount of head movement; no significant differences were found in the mean, standard deviation and maximum of the movement parameters. Subjects were excluded when head movements exceeded $1.5 \mathrm{~mm} / \mathrm{s}$ or 1.5 degrees $/ \mathrm{s}$ in at least one direction. Data of nine patients were excluded from further analysis because of movement related artifacts ( $n=6 ; 2$ controls, 4 patients) or EPI artifacts ( $n=3 ; 2$ controls, 1 patient). The final study population for analysis included 30 patients and 37 healthy controls. 


\section{Cognition}

Six patients did not complete the neuropsychological assessment and had no CVST scores. These patients are included in the group analysis, but not in the correlation analysis. In total, 11 FLE patients had a decile score below 4 and were considered cognitively impaired. Mean CVST reaction time was significantly higher in the patient group ( $\mathrm{N}=37$ ) (controls: $17.3 \pm 6.4 \mathrm{~s}$, patients: $23.8 \pm 9.5 \mathrm{~s}, p<0.002$ ).

\section{Between-group analysis of network parameters}

The mean matrix weight of the weighted connection matrices did not differ significantly between the control and patient group, nor between the control and impaired patient group. Network parameters were assessed over a range of sparsity values (0.35 to 0.75 ). Both patient and control networks showed small-world properties indicated by a high clustering compared to equivalent random networks (mean $C=1.82$, range 1.19-4.65) and a path length comparable to equivalent random networks (mean $L=1.02$, range 1.00-1.09) (Watts and Strogatz, 1998). The binary cluster coefficient displayed significantly higher values in the cognitively impaired patient group compared to the control group for the relatively small sparsity range 0.67-0.74. The binary path length was significantly higher in the impaired patient group compared to the control group for the narrow sparsity range $0.41-0.47$. The weighted cluster coefficient was significantly higher for the impaired patient group compared to the healthy control group over the sparsity range 0.37-0.46 and 0.650.85 . The weighted path length was significantly higher for the impaired patient group compared to the healthy control group over the sparsity range 0.64-0.74.

In contrast to $L$ and $C$, we found that modularity showed significant group differences over a much wider range of sparsity values. The modularity calculated from the binary networks was higher in the entire patient group compared to the control group for the sparsity range $0.58-0.73$. The impaired patient group displayed significantly higher modularity compared to the control group for the entire sparsity range. The impaired patient group also showed higher modularity scores compared to the non-impaired patient group over the sparsity range $0.37-0.70$. The weighted modularity scores were higher for both the entire patient group and the impaired patient group compared to the control group over the entire sparsity range. These results are visualized in Figure 5.2 .

\section{Network metrics, age and cognitive performance}

Within the patient group, we found that binary modularity scores significantly increased with decreased cognitive performance (i.e. increased CVST reaction time) for all sparsity values (mean $r=0.48$; range: $0.44-0.55$; all $p$-values $<0.03$ ). See Figure 5.3 for a plot of the correlation between CVST reaction times and modularity scores at sparsity $=0.48$. The same effect was found with the CVST decile scores, which is a 
normalized age and gender corrected score (mean $r=-0.55$; range: $-0.63--0.47$; all $p$ values $<0.02$ ). Higher modularity scores were associated with longer reaction times and lower decile scores (i.e. poor performance). CVST reaction times positively correlated with the weighted modularity scores over the entire sparsity range (mean $r=0.47$; min-max: $0.43-0.54$; all $p$-values $<0.05$ ). CVST decile scores were negatively correlated with the weighted modularity scores over the entire sparsity range (mean $r=-0.45$; range $-0.55--0.40$; all $p$-values $<0.05$ ). No significant correlations were found in the control group. No significant correlations were found between age (range 8-12 y) and any of the network parameters. CVST reaction times and decile scores were not significantly correlated with the binary or weighted cluster coefficient or path length in the patient or control groups.
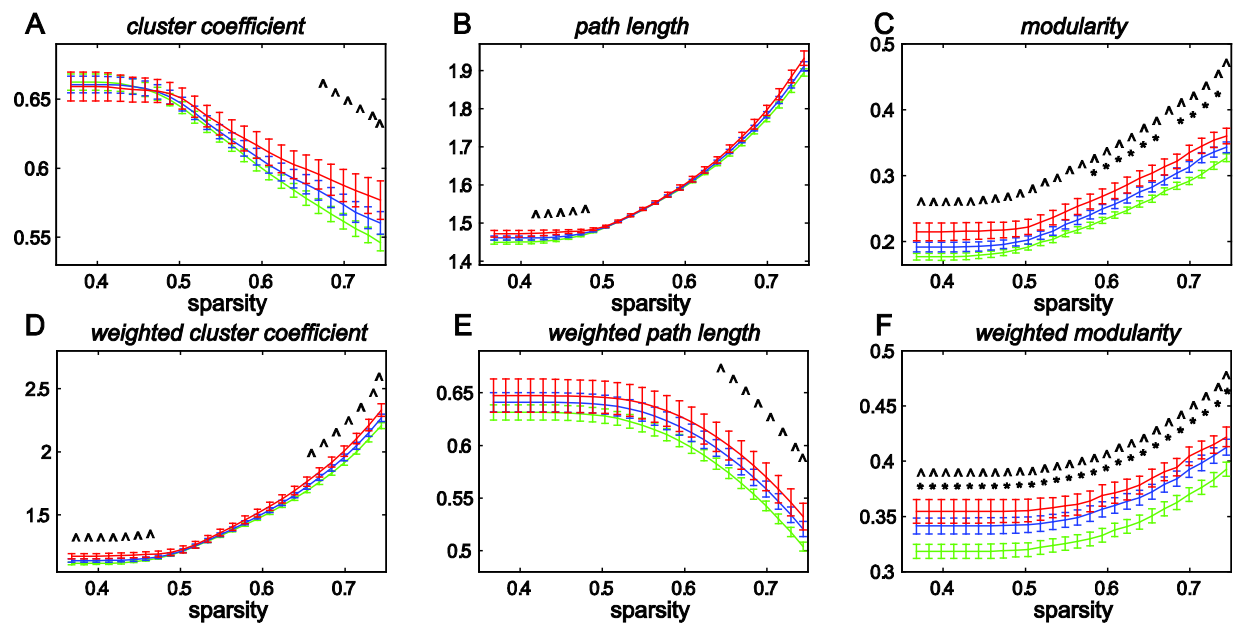

Figure 5.2 The mean \pm standard error (error bars) of the tested network parameters for the control group (green), the full patient group (blue) and the cognitively impaired patient group (red) as function of sparsity. An asterisk $\left(^{*}\right)$ indicates that the full patient group was significantly different from the control group. A hat $(\wedge)$ indicates that the impaired patient group was significantly different from the control group. As the sparsity increases, the number of edges in the network decreases, which causes a decrease in the binary cluster coefficient (A) and an increase in the binary path length (B) and binary and weighted modularity scores (C and F). The weighted cluster coefficient increases (D) and the weighted path length increases (E) because the remaining edges have high connection strengths and are strongly clustered (D). 


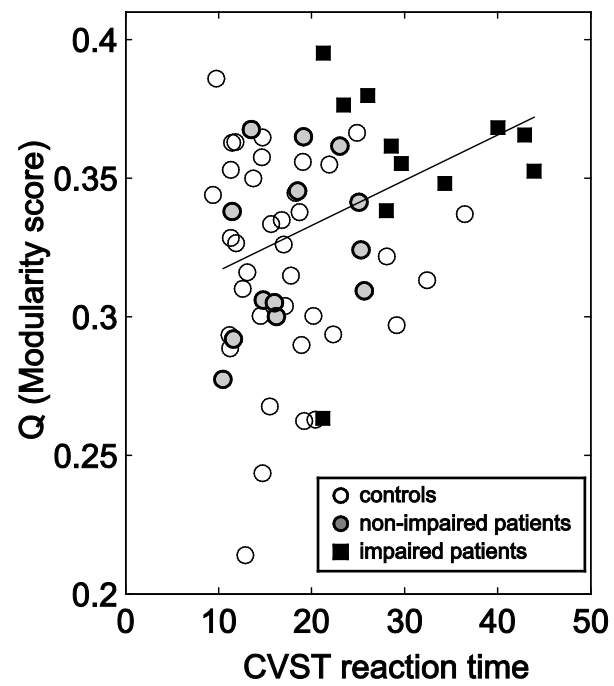

Figure 5.3 Scatter plot of the modularity scores against CVST scores for the control, entire patient and impaired patient group. The regression line (black) of the correlation between modularity and CVST for the (entire) patient group is displayed.

\section{Visualization of cerebral modularity}

The OCS was calculated for networks thresholded at sparsity $=0.48$. About half of the possible number of edges (3500 out of 6724) in the full matrix is included at this sparsity value, which reflects a good balance between the presence of noisy edges and an overly sparse matrix.

Four modules were found in all groups at this sparsity threshold. All modules were organized in a bilateral fashion. Figure 5.4 visualizes the organization of the OCS in the cerebrum, such that group differences in modules can be observed. Considering the control group, module 1 was located mainly in the frontal and parietal lobe (blue in Figure 5.4), module 2 was mainly located in the frontal lobe (red in Figure 5.4), module 3 was mainly located in the occipital lobe (yellow in Figure 5.4), while module 4 was mainly located in the frontal and temporal lobes (green in Figure 5.4). Both the patient group as a whole and the cognitively impaired patients displayed several differences in modular structure in both hemispheres in comparison to the control group. Module 4 (the green module), occupies occipital, parietal, temporal and prefrontal regions in the control group, whereas this module curtails only to temporal regions in the impaired patient group. As a consequence module 1 (blue) occupies most of the prefrontal regions and therefore the diversity of the modular composition in the frontal lobe decreases in the entire patient group, and even further decreases in the cognitively impaired patient group relative to the control group (see 
Supplementary data). Although the vast majority of modular differences were located in frontal regions ( $n=21)$, as could be expected considering the frontal seizure focus in FLE, parietal $(n=8)$, temporal $(n=2)$ and occipital $(n=2)$ regions are also involved.

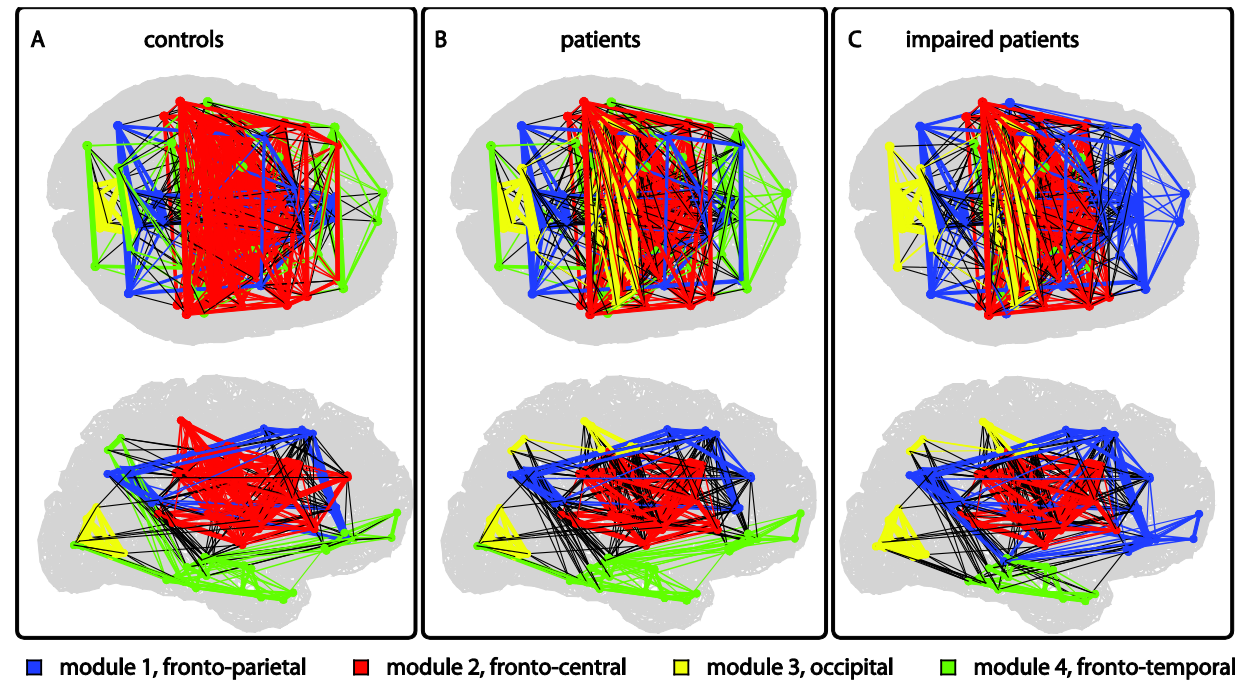

Figure 5.4 In the Optimal Community Structure (OCS) four distinct modules of the cerebrum are visualized by different colors (as in Figure 5.1) for the healthy controls (A), the full patient group (B), and the cognitively impaired patient subgroup. Module 1 (blue) extends from fronto-parietal regions in controls to more prefrontal and latero-frontal regions in patients, particularly for the cognitively impaired patients. Module 2 (red) reveals no apparent differences between patients and controls. Module 3 (yellow) extends from mere posterior occipital regions in controls to parietal and more latero-occipetal regions in patients. Module 4 (green) curtails from occipital, parietal, temporal and frontal regions to temporal and frontal regions.

\section{Modular and distance based characteristics of aberrant connections}

As shown in Figure 5.5A, a number of connections were found to be altered in the patient group, and a larger number of aberrant connections was found in the impaired patient group. These results were obtained at the same sparsity threshold as the one used for visualization of the modules (sparsity=0.48). Of the connections significantly different at the $p<0.05$ level, 52 (41\%) were intra-modular connections while 151 (59\%) were inter-modular connections. Moreover, Figure 5.5B indicates that most connections weaker in patients $(p<0.05)$ are oriented anterior-posteriorly, while the connections stronger in the patients are mainly oriented left-right and interhemispheric. This discrepancy in orientation was quantified by measuring the angle of the connections with respect to left-right axis (a 90 degree angle would indicate a pure anterior-posterior orientation of an edge). This revealed that the connections weaker in patients had a significantly higher angle (hence were oriented more anterior-posteriorly) than the connections stronger in patients $(65.3 \pm 22.2$ degrees vs. 
$38.8 \pm 27.5$ degrees, $p<0.001)$. We tested whether the anatomical distance between the connections that were either stronger or weaker would differ. We found that the connections that were weaker in the patient group were on average longer $(76.8 \pm$ $26.9 \mathrm{~mm})$ than those that were stronger in the patient group $(65.7 \pm 26.0 \mathrm{~mm}$, $p<0.003)$, see Figure 5.5C.

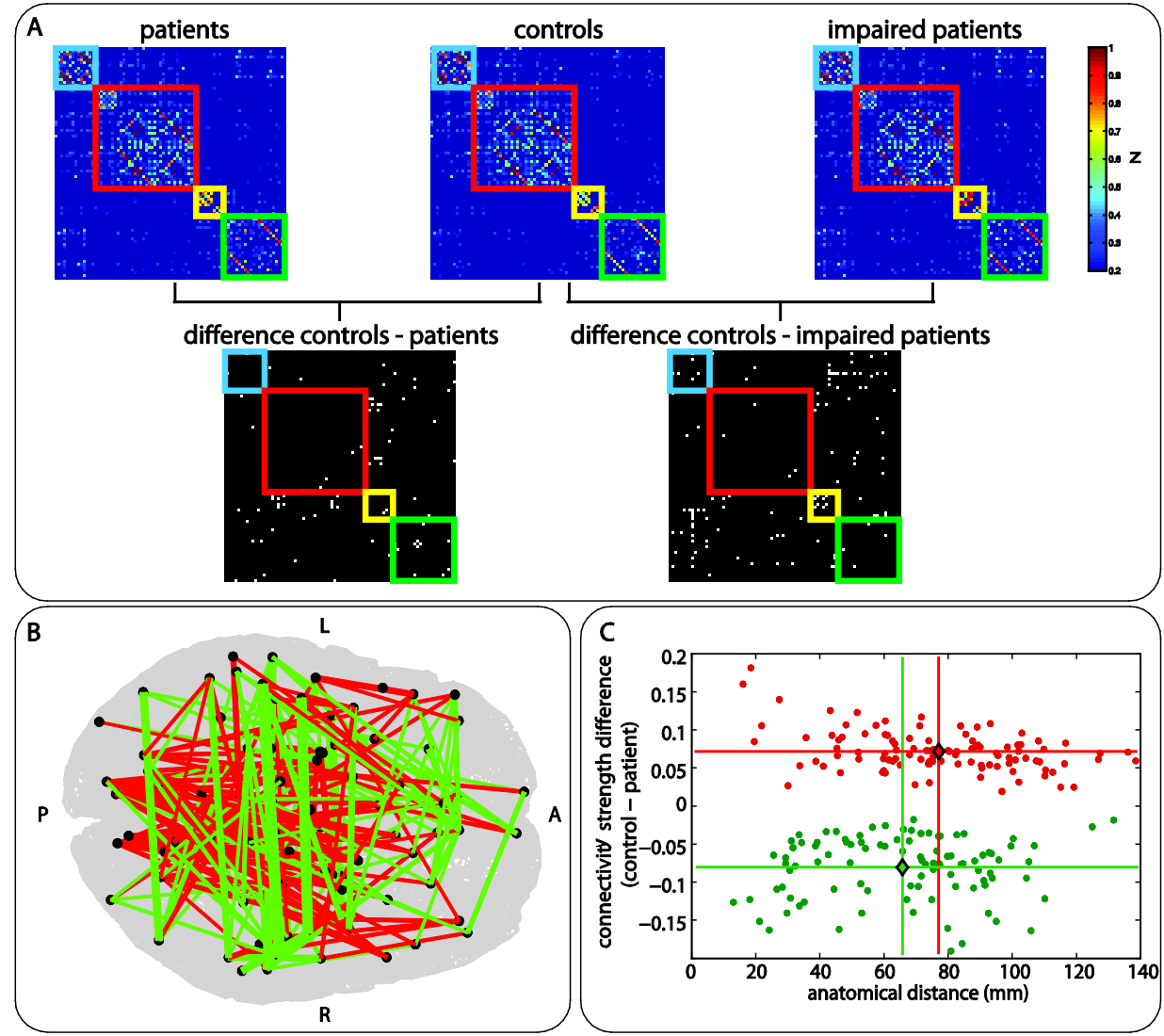

Figure 5.5 (A) Top row: connectivity matrices for all three groups; controls (middle), patients (left) and impaired patients (right). Colors of matrix elements indicate Fisher z-transformed correlation coefficients (truncated between 0.2 and 1 ). The rows and columns of the matrices are sorted by the modules found in the control group as indicated by the colored squares. Bottom row shows the connections that differed at the $p<0.01$ significance level (uncorrected) between the control and patient (left) and impaired patients (right). Note that most aberrant connections are inter-modular. (B) Location of abnormal connections at the $p<0.05$ level. Red and green lines indicate connections weaker and stronger, respectively, in patients. Red (weaker) connections are oriented in an anterior-posterior fashion, while green (stronger) connections have a left-right orientation (C). Same set of connections as in (B) versus anatomical distance. On average, the connections weaker in patients (red dots) are longer than the connections stronger in patients (green dots). Red and green diamonds and lines indicate the average (distance and difference) of the two classes of connections. 


\section{DISCUSSION}

Here, we have shown for the first time that a neuronal correlate for cognitive impairment exists in children with FLE. Our results suggest that functional networks in FLE are configured to have reduced connectivity between functional modules with a decline in long-range connectivity and an increase in inter-hemispheric connectivity. This was expressed by an increased modularity score in paediatric patients with epilepsy that was correlated with the cognitive impairment. Notably, in the cognitively impaired patients, the frontal lobe missed the characteristic module that functionally interacted with the temporal, parietal and occipital regions as seen in the healthy controls.

Interestingly, the discrepancy in network organization found between the children with FLE (with cognitive impairments) and normal controls has previously been reported in healthy development, where a decrease in modularity and an increase in long-range connectivity was associated with normal brain maturation (Fair et al., 2009; Hagmann et al., 2010).

Our results relate to a larger body of literature on the relation between cognitive performance and large-scale connectivity, where it is suggested that higher cognitive functions are the result of interactions between systems involving numerous brain regions, instead of a direct relation between cognitive functioning and single brain regions (Bressler and Menon, 2010; Menon, 2011). This paradigm can also be extended to differences between healthy subjects and FLE patients. The brain and especially the frontal lobe is a highly connected structure and thus regional abnormalities might extend beyond the seizure focus and affect distant regions and connectivity to such regions. Functional MRI measurements are ultimately dependent on the synaptic and axonal configuration of the underlying neuronal ensembles. However, it is thought that, by approximately 9 month of age, axonal connectivity is near complete (Conel, 1947) but other mechanisms such as synaptic pruning (Huttenlocher, 1979) and axonal myelination (Fields, 2005) continue through young adulthood. How then do epileptic seizures (Dodrill, 2002) and daily AED use (Vermeulen and Aldenkamp, 1995; Kuhnert et al., 2010) interact with the mechanisms of normal development and how do they eventually affect development of large-scale brain connectivity as measured with fMRI? It is likely that disturbances early in life in any of these mechanisms may have profound influences on large parts of the brain, as indicated by the whole brain network results presented here.

\section{Previous findings}

Several studies found functional connectivity abnormalities in epilepsy by means of calculating correlation coefficients between pairs of brain regions (Waites et al., 2006; Bettus et al., 2009; Zhang et al., 2009a; Zhang et al., 2009b; Pereira et al., 2010; Wang et al., 2011), but in only one study an analysis of these correlations was performed in 
terms of network parameters (Liao et al., 2010). Moreover, most of these studies focused on local connectivity abnormalities (only a few regions of the brain were analysed or considered as a reference), while here, we primarily analysed global brain connectivity. Cognitive functioning depends on several cerebral networks instead of isolated brain regions. It is reasonable to assume that in patients with epilepsy a disruption of whole brain networks is involved in the development of cognitive deficits, instead of a localized disruption at the site of seizure focus only (Vlooswijk et al., 2011).

\section{Increased modularity but preserved small-worldness in FLE}

The means of both variants of the cluster coefficient were higher in the patients and further increased in the impaired patient group. For both variants of the path length, the same effect could be observed. Networks with high path length and high clustering are also known as regular networks (Sanz-Arigita et al., 2010): these are networks with high local clustering but few connections linking distant nodes. These findings are in line with the modularity analysis: high path length and high clustering are signs that the patient networks are organized in tightly clustered modules with only limited inter-modular connectivity. However, the high clustering and comparable low path length, compared to equivalent random networks, indicate that the resting state functional networks of both groups are still organized as an efficient small world network (Stam et al., 2007). Previous studies have found altered small world networks in epilepsy patients (Liao et al., 2010; Vlooswijk et al., 2011). It remains to be elucidated why these parameters only showed limited effects in this study.

The network parameter modularity did show ample significant group differences. Patients, especially the cognitively impaired patients, showed higher modularity scores than controls, suggesting the presence of more functionally isolated brain modules. In line with these findings, longer reaction times (greater cognitive impairment) correlated with higher modularity scores within the patient group. It is possible that increases in coherent activity between functional systems (integration) might facilitate particular cognitive abilities. Therefore, a reduced amount of integration could lead to an impairment of cognitive functions.

When we visualized the modular structures of different subject groups (Figure 5.4) we observed a rearrangement of modular structures between controls and patients, which was more pronounced in the cognitively impaired subgroup. These findings suggest that disruptions of functional brain network modularity in children with cryptogenic FLE are related to their cognitive impairment. Furthermore, the vast majority of modular differences were located in frontal lobe, as could be expected considering the frontal seizure focus in FLE. Importantly, module 4 (green) comprised prefrontal, temporal, parietal and occipital regions and might thus facilitate information integration over spatially distributed regions of the brain. Especially this module curtailed to mere temporal regions in the impaired patient group. The 
functional significance of this module remains to be elucidated, but the corresponding connections to the frontal lobe might be an interesting target for future studies. The finding that modular abnormalities did not seem to be restricted exclusively to the frontal lobe might imply that regions of other parts of the brain are also involved in the process that hinder some individuals with FLE to successfully perform complex cognitive tasks like the CVST. This could also be an explanation for the broad variety of cognitive impairment seen in children with FLE.

\section{Aberrant connections and anatomical distance}

We found both decreases and increases in connection strengths in the patient group (figure 5.5B), although given the large number of connections tested, these findings should be interpreted with care. A further analysis on the anatomical length and orientation of these connections did reveal an interesting effect. Connections with decreased strength in the patients were on average longer than the connections that were increased in the patients, compared to the healthy controls. Furthermore, a predominantly anterior-posterior orientation of the connections with decreased strength and a left-right (and thus inter-hemispheric) orientation of connections with increased strength in the patients was found. This is in agreement with prior studies that showed that in early (normal) development mainly the long range connections increase in strength, while the strength of the short range connections decreased (Fair et al., 2009; Supekar et al., 2009; Hagmann et al., 2010). This raises the question of whether FLE interferes with normal development of functional brain networks (Power et al., 2010; Uddin et al., 2010). However, due to the narrow age range of our study population, network metrics could not be related to age. Future studies should include FLE patients and controls with a wider age range to investigate whether cognitive impairment in FLE can be modeled as a developmental delay (Church et al., 2009). Most connections that showed significant differences between the healthy control and patients were inter-modular connections. These are connections that contribute to the overall integration of functional systems in the brain. These findings indicate that the higher modularity scores found in the patient and impaired patient group can be mainly attributed to connectional differences in those regions that connect different modules.

\section{Clinical significance and future research}

Follow-up research is needed to investigate the relationship between cognition and measures of network topology, particularly for determining the prognostic value of these measures that predict cognitive progress or delay in time. Currently, no clinical tools are available that can reliably predict the long-term cognitive outcome and drug response in children with FLE. Individual connectivity maps and network analysis might eventually serve as an additional tool for the neurologist to tailor the 
therapeutic decision-making to the network characteristics of the impaired brain, and to balance this to seizure treatment.

\section{Methodological considerations}

Several considerations in the choice of methodology for the current study should be discussed. First, the use of resting state fMRI has advantages over task fMRI because the experiment is not dependent on subject compliance and ability to perform a task. This can especially be relevant in paediatric studies, where differences in developmental status and task performance are present. Here, all included subjects were video monitored during scanning and were able to lie still with their eyes closed. Second, there are many algorithms designed to calculate the OSC (Meunier et al., 2010). Although we applied a widely-used algorithm (Newman, 2006), novel approaches for comparing the OCS between several different individual networks (Meunier et al., 2009), or groups of networks (Alexander-Bloch et al., 2010), have also been proposed. Other authors have suggested that future algorithms might include the concept of persistence of information flowing within modules over time (Delvenne et al., 2010), or use the concept of hierarchy, subdividing the modules into smaller modules, which can be further subdivided into smaller modules, and so on (Meunier et al., 2010). In the latter method, large modules have been described to represent consciously demanding tasks (i.e., working memory or the cognitive task from the current study), because they demand access to a more globally integrated processing system (Zeki and Bartels, 1998). Smaller modules are supposed to represent automated, anatomically localized tasks (i.e., color vision or visual motion detection). Because consciously demanding tasks usually include a combination of automated tasks, algorithms that determine the modular structure on different levels might be useful in future research relating cerebral connectivity data to cognition.

\section{Conclusion}

In conclusion, our results show that network modularity analysis of whole brain resting state $\mathrm{fMRI}$ connectivity provides a sensitive marker for cognitive impairment in FLE. We found that the more cognitively impaired the FLE-patient is, the more isolated brain sub-networks appeared to function. Cognitively impaired patients seem to have a less efficient inter-regional transfer of information between functional networks. We suggest that abnormally interconnected functional sub-networks of the brain might underlie the cognitive problems in children with FLE. 


\section{REFERENCES}

Achard S, Bullmore E (2007) Efficiency and cost of economical brain functional networks. PLoS Comput Biol 3:e17.

Aldenkamp AP, Ar ends J, Verspeek S, Berting M (2004) The cognitive impact of epileptiform EEG-discharges; relationship with type of cognitive task. Child Neuropsychol 10:297-305.

Alexander-Bloch AF, Gogtay N, Meunier D, Birn R, Clasen L, Lalonde F, Lenroot R, Giedd J, Bullmore ET (2010) Disrupted modularity and local connectivity of brain functional networks in childhoodonset schizophrenia. Front Syst Neurosci 4:147.

Bettus G, Guedj E, Joyeux F, Confort-Gouny S, Soulier E, Laguitton V, Cozzone PJ, Chauvel P, Ranjeva JP, Bartolomei F, Guye M (2009) Decreased basal fMRI functional connectivity in epileptogenic networks and contralateral compensatory mechanisms. Hum Brain Mapp 30:1580-1591.

Braakman HM, Vaessen MJ, Hofman PA, Debeij-van Hall MH, Backes WH, Vles JS, Aldenkamp AP (2011) Cognitive and behavioral complications of frontal lobe epilepsy in children: A review of the literature. Epilepsia 52:849-856.

Bressler SL, Menon V (2010) Large-scale brain networks in cognition: emerging methods and principles. Trends in cognitive sciences 14:277-290.

Centeno M, Thompson PJ, Koepp MJ, Helmstaedter C, Duncan JS (2010) Memory in frontal lobe epilepsy. Epilepsy Res 91:123-132.

Church JA, Fair DA, Dosenbach NU, Cohen AL, Miezin FM, Petersen SE, Schlaggar BL (2009) Control networks in paediatric Tourette syndrome show immature and anomalous patterns of functional connectivity. Brain 132:225-238.

Conel JLR (1947) The postnatal development of the human cerebral cortex: The cortex of the three-month infant: Harvard University Press.

Delvenne JC, Yaliraki SN, Barahona M (2010) Stability of graph communities across time scales. Proc Natl Acad Sci U S A 107:12755-12760.

Dodrill CB (2002) Progressive cognitive decline in adolescents and adults with epilepsy. Prog Brain Res 135:399-407.

Fair DA, Cohen AL, Power JD, Dosenbach NU, Church JA, Miezin FM, Schlaggar BL, Petersen SE (2009) Functional brain networks develop from a "local to distributed" organization. PLoS Comput Biol 5:e1000381.

Fields RD (2005) Myelination: an overlooked mechanism of synaptic plasticity? The Neuroscientist : a review journal bringing neurobiology, neurology and psychiatry 11:528-531.

Filippini N, Maclntosh BJ, Hough MG, Goodwin GM, Frisoni GB, Smith SM, Matthews PM, Beckmann CF, Mackay CE (2009) Distinct patterns of brain activity in young carriers of the APOE-epsilon4 allele. Proc Natl Acad Sci U S A 106:7209-7214.

Fox MD, Raichle ME (2007) Spontaneous fluctuations in brain activity observed with functional magnetic resonanceimaging. Nat Rev Neurosci 8:700-711.

Friston KJ (1994) Functional and effective connectivity in neuroimaging: A synthesis. Human Brain Mapping 2:56-78.

Ghosh SS, Kakunoori S, Augustinack J, Nieto-Castanon A, Kovelman I, Gaab N, Christodoulou JA, Triantafyllou C, Gabrieli JD, Fischl B (2010) Evaluating the validity of volume-based and surfacebased brain image registration for developmental cognitive neuroscience studies in children 4 to 11 years of age. Neuroimage 53:85-93. 
Ginestet CE, Nichols TE, Bullmore ET, Simmons A (2011) Brain network analysis: separating cost from topology using cost-integration. PLoS One 6:e21570.

Hagmann P, Sporns O, Madan N, Cammoun L, Pienaar R, Wedeen VJ, Meuli R, Thiran JP, Grant PE (2010) White matter maturation reshapes structural connectivity in the late developing human brain. Proc Natl Acad Sci U S A 107:19067-19072.

Harvey AS, Hopkins IJ, Bowe JM, Cook DJ, Shield LK, Berkovic SF (1993) Frontal lobe epilepsy: clinical seizure characteristics and localization with ictal 99mTc-HMPAO SPECT. Neurology 43:1966-1980.

Huttenlocher PR (1979) Synaptic density in human frontal cortex - developmental changes and effects of aging. Brain research 163:195-205.

Kuhnert MT, Elger CE, Lehnertz K (2010) Long-term variability of global statistical properties of epileptic brain networks. Chaos 20:043126.

Laskowitz DT, Sperling MR, French JA, O'Connor MJ (1995) The syndrome of frontal lobe epilepsy: characteristics and surgical management. Neurology 45:780-787.

Lawson JA, Cook MJ, Vogrin S, Litewka L, Strong D, Bleasel AF, Bye AM (2002) Clinical, EEG, and quantitative MRI differences in pediatric frontal and temporal lobe epilepsy. Neurology 58:723-729.

Liao W, Zhang Z, Pan Z, Mantini D, Ding J, Duan X, Luo C, Lu G, Chen H (2010) Alter ed functional connectivity and small-world in mesial temporal lobe epilepsy. PLoS One 5:e8525.

Lorenzo NY, Parisi JE, Cascino GD, Jack CR, Jr., Marsh WR, Hirschorn KA (1995) Intractable frontal lobe epilepsy: pathological and MRI features. Epilepsy Res 20:171-178.

Manford M, Hart YM, Sander JW, Shorvon SD (1992) National General Practice Study of Epilepsy (NGPSE): partial seizure patterns in a general population. Neurology 42:1911-1917.

Maslov S, Sneppen K (2002) Specificity and stability in topology of protein networks. Science 296:910-913.

Menon V (2011) Large-scale brain networks and psychopathology: a unifying triple network model. Trends in cognitive sciences 15:483-506.

Meunier D, Lambiotte R, Bullmore ET (2010) Modular and hierarchically modular organization of brain networks. Front Neurosci 4:200.

Meunier D, Lambiotte R, Fornito A, Ersche KD, Bullmore ET (2009) Hierarchical modularity in human brain functional networks. Front Neuroinformatics 3:37.

Newman ME (2006) Modularity and community structure in networks. Proc Nat Acad Sci U S A 103:85778582.

Patrikelis P, Angelakis E, Gatzonis S (2009) Neurocognitive and behavioral functioning in frontal lobe epilepsy: a review. Epilepsy Behav 14:19-26

Pereira FR, Alessio A, Sercheli MS, Pedro T, Bilevicius E, Rondina JM, Ozelo HF, Castellano G, Covolan RJ, Damasceno BP, Cendes $\mathrm{F}$ (2010) Asymmetrical hippocampal connectivity in mesial temporal lobe epilepsy: evidence from resting state fMRI. BMC Neurosci 11:66.

Power JD, Fair DA, Schlaggar BL, Petersen SE (2010) The development of human functional brain networks. Neuron 67:735-748.

Pravata E, Sestieri C, Mantini D, Briganti C, Colicchio G, Marra C, Colosimo C, Tartaro A, Romani GL, Caulo M (2011) Functional connectivity MR imaging of the language network in patients with drugresistant epilepsy. AJNR Am J Neuroradiol 32:532-540.

Prevost J, Lortie A, Nguyen D, Lassonde M, Carmant L (2006) Nonlesional frontal lobe epilepsy (FLE) of childhood: clinical presentation, response to treatment and comorbidity. Epilepsia 47:21982201.

Provini F, Plazzi G, Tinuper P, Vandi S, Lugaresi E, Montagna P (1999) Nocturnal frontal lobe epilepsy. A clinical and polygraphic overview of 100 consecutive cases. Brain 122 ( Pt 6):1017-1031. 
Rubinov M, Sporns O (2010) Complex network measures of brain connectivity: uses and interpretations. Neuroimage 52:1059-1069.

Sanz-Arigita EJ, Schoonheim MM, Damoiseaux JS, Rombouts SA, Maris E, Barkhof F, Scheltens P, Stam CJ (2010) Loss of 'small-world' networks in Alzheimer's disease: graph analysis of FMRI resting-state functional connectivity. PLoS One 5 :e13788.

Stam CJ, Jones BF, Nolte G, Breakspear M, Scheltens P (2007) Small-world networks and functional connectivity in Alzheimer's disease. Cereb Cortex 17:92-99.

Supekar K, Musen M, Menon V (2009) Development of large-scale functional brain networks in children. PLoS Biol 7:e1000157.

Uddin LQ, Supekar K, Menon V (2010) Typical and atypical development of functional human brain networks: insights from resting-state FMRI. Front Syst Neurosci 4:21.

Vaessen MJ, Hofman PA, Tijssen HN, Aldenkamp AP, Jansen JF, Backes WH (2010) The effect and reproducibility of different clinical DTI gradient sets on small world brain connectivity measures. Neuroimage 51:1106-1116.

Van den Heuvel MP, Hulshoff Pol HE (2010) Exploring the brain network: a review on resting-state fMRI functional connectivity. Eur Neuropsychopharmacol 20:519-534.

van den Heuvel MP, Stam CJ, Kahn RS, Hulshoff Pol HE (2009) Efficiency of functional brain networks and intellectual performance. J Neurosci 29:7619-7624.

Van Dijk KRA, Hedden T, Venkataraman A, Evans KC, Lazar SW, Buckner RL (2010) Intrinsic Functional Connectivity As a Tool For Human Connectomics: Theory, Properties, and Optimization. Journal of Neurophysiology 103:297-321.

Vermeulen J, Aldenkamp AP (1995) Cognitive side-effects of chronic antiepileptic drug treatment: a review of 25 years of research. Epilepsy Res 22:65-95.

Vlooswijk MC, Jansen JF, Majoie HJ, Hofman PA, de Krom MC, Aldenkamp AP, Backes WH (2010) Functional connectivity and language impairment in cryptogenic localization-related epilepsy. Neurology 75:395-402.

Vlooswijk MC, Vaessen MJ, Jansen JF, de Krom MC, Majoie HJ, Hofman PA, Aldenkamp AP, Backes WH (2011) Loss of network efficiency associated with cognitive decline in chronic epilepsy. Neurology 77:938-944.

Waites AB, Briellmann RS, Saling MM, Abbott DF, Jackson GD (2006) Functional connectivity networks are disrupted in left temporal lobe epilepsy. Ann Neurol 59:335-343.

Wang Z, Lu G, Zhang Z, Zhong Y, Jiao Q, Tan Q, Tian L, Chen G, Liao W, Li K, Liu Y (2011) Altered resting state networks in epileptic patients with generalized tonic-clonic seizures. Brain Res 1374:134-141.

Watts DJ, Strogatz SH (1998) Collective dynamics of 'small-world' networks. Nature 393:440-442.

Zeki S, Bartels A (1998) The autonomy of the visual systems and the modularity of conscious vision. Philos Trans R Soc Lond B Biol Sci 353:1911-1914.

Zhang Z, Lu G, Zhong Y, Tan Q, Liao W, Chen Z, Shi J, Liu Y (2009a) Impaired perceptual networks in temporal lobe epilepsy revealed by resting fMRI. J Neurol 256:1705-1713.

Zhang Z, Lu G, Zhong Y, Tan Q, Yang Z, Liao W, Chen Z, Shi J, Liu Y (2009b) Impaired attention network in temporal lobe epilepsy: a resting FMRI study. Neurosci Lett 458:97-101. 


\section{SUPPLEMENTARY DATA}
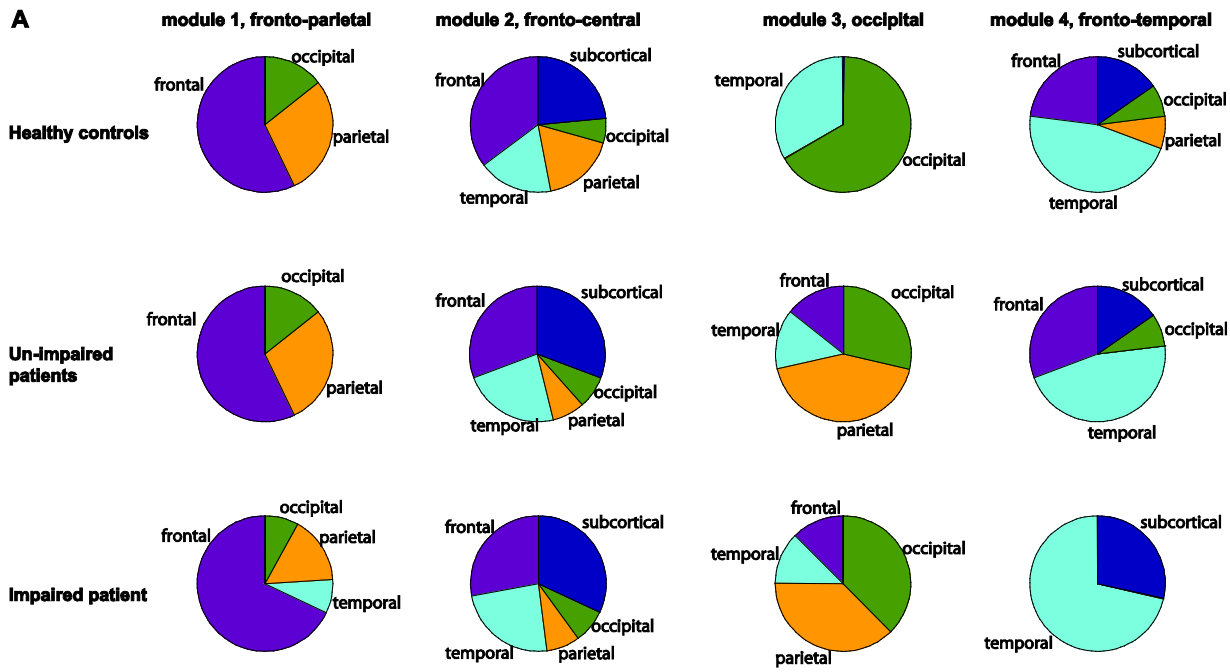

B

Healthy controls
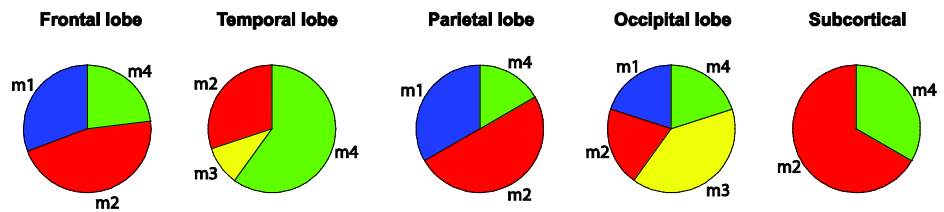

Un-Impalred patlents
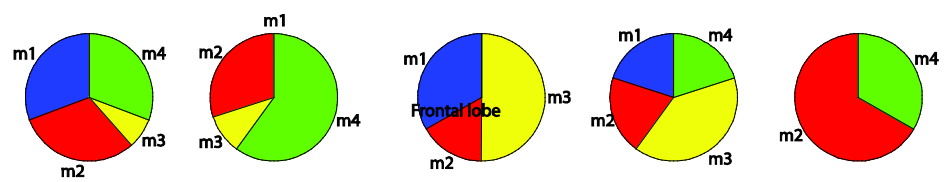

Impalred patlent
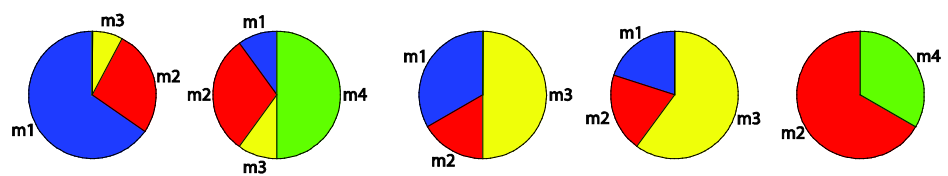

Figure S.5.1 (A) The lobar composition of each module is depicted as a pie chart showing the relative number of regions (nodes) of each lobe to a module. For instance, module 3 is composed of only temporal and occipital regions in the control group, while parietal and frontal regions are also present in the patient groups. (B) The modular composition of each lobe is depicted here in the same fashion as in (A). The frontal lobe is occupied by regions from modules 1,2 and 4 in the control group, while in the impaired patient group, modules 1,2 and 3 are found in the frontal lobe. The same color schemes as in Figure 5.1 are used. 


\section{CHAPTER 6}

Connectional abnormalities of functional and structural networks in childhood frontal lobe epilepsy

M.J. Vaessen, H.M.H. Braakman, P.A.M. Hofman, A. de Louw, A.P. Aldenkamp, J.F.A. Jansen, W.H. Backes In preparation 


\section{ABSTRACT}

In childhood frontal lobe epilepsy (FLE) cognitive impairment and educational underachievement are serious well-known co-morbid disorders. The broad scale of affected cognitive domains suggests a global network disturbance, rather than perturbations of localized individual processes. In this study we have investigated whole brain connectional properties of children with FLE in relation to their cognitive impairment and compared them with healthy controls. Functional connectivity (FC) of the networks was derived from dynamic fluctuations of resting state $\mathrm{fMRI}$ and structural connectivity (SC) was obtained from fiber tractography. Graph theoretical analysis was used to characterize the whole brain network in terms of path length, clustering and the degree to which the network can be separated into modules. Subsequently, the connectivity within and between modules was related to cognitive performance. Functional network disturbances in FLE comprised increased clustering, increased path length, and stronger modularity compared to healthy controls, which was accompanied by stronger within and weaker between-module functional connectivity. Although structural path length and clustering appeared normal in children with FLE, structural modularity increased with stronger cognitive impairment. It is concluded that decreased coupling between large-scale functional network modules is a hallmark for impaired cognition in childhood FLE. 


\section{INTRODUCTION}

Frontal lobe epilepsy (FLE) is considered to be, after temporal lobe epilepsy, the second most common type of the localization-related (partial) epilepsies of childhood and accounts for 20-30\% of partial epilepsies (Manford et al., 1992). Pediatric FLE, even when cryptogenic in nature, is frequently complicated by the impairment of a broad range of cognitive problems, behavioral disturbances, and therapy resistance (Berg, 2011). The fact that all these complications occur at a young age is troublesome. In childhood the brain is at its most vulnerable state and neurologic disturbances such as FLE can have an impact on brain maturation and the development of cognitive skills, with potentially severe consequences for school performance (Braakman et al., 2011).

The broad range of affected cognitive domains suggests a global network disturbance, rather than perturbations of localized individual processes. Disturbances in network organization can be assessed by connectome analysis, which comprises the mapping of the nodes and connections of the human cerebral network (Sporns, 2011). Cerebral connectivity may either be of functional or structural nature. Functional connectivity (FC) can be measured by correlating blood-oxygen-dependent oxygenation (BOLD) related dynamic fluctuations of gray matter activity between different brain regions (Fox and Raichle, 2007) and structural connectivity (SC) can be obtained by tracing axonal bundles through the white matter with fiber tractography (Tournier et al., 2011).

Resting state functional MRI (RS-fMRI) enables the investigation of the intrinsic functional organization of the brain and is typically measured by the temporal correlation of neuronal activity-induced signal variations of anatomically different brain regions (Friston, 1994; Van den Heuvel and Hulshoff Pol, 2010). Previous studies have demonstrated disruptions in functional networks of adult epilepsy patients (Waites et al., 2006; Liao et al., 2010; Pereira et al., 2010), which have also been related to cognitive and epilepsy variables (Bettus et al., 2009; Vlooswijk et al., 2010; Pravata et al., 2011; Vlooswijk et al., 2011).

Modeling the brain as one system of nodes (brain regions) and edges (connections) allows a direct comparison of SC and FC, because the organization of nodes and edges can be derived from both functional and structural imaging data. Apart from correlating the functional and structural connection strengths of individual edges, one can also explore and relate the topology of SC and FC networks in terms of graph theoretical measures. Graph theoretical analysis has the advantage that topological properties of the whole brain network can be captured in a few summary measures that describe the amount of segregation and integration among brain regions (Stam and Reijneveld, 2007; Bullmore and Sporns, 2009).

Changes in either FC or SC are interdependent (Johansen-Berg, 2011). However, the relation between FC and SC is likely to be complex (Honey et al., 2010). The white matter connectivity provides a physical substrate that possibly constraints the 
functional connectivity between different brain regions (Honey et al., 2009; Hagmann et al., 2010; Ethofer et al., 2011). Several studies have indicated that SC is predictive for FC, while FC is not predictive for SC, across healthy human brain networks (Greicius et al., 2009; Honey et al., 2009). The SC-FC relation increases in strength during normal development (Hagmann et al., 2010) and might be disrupted in the diseased brain (Skudlarski et al., 2010; Zhang et al., 2011). However, it is unclear to what extend abnormalities in the dependency between FC and SC explains cognitive impairment.

Previously, it was observed that the neuronal basis for cognitive deficits in FLE reside in the interaction between large-scale functional brain sub networks, the so-called modules (Vaessen, 2011). The whole brain network can be divided into sub networks by modular decomposition (i.e. community structure) methods (Newman, 2006), and therefore, this method provides the opportunity to investigate the connectional properties of the different large-scale sub networks. The modular structure of the brain network is thought to be important for cognitive abilities, as increases in coherent activity between functional systems might facilitate adaptive behavior and the integration of information integration (van den Heuvel et al., 2009; Power et al., 2010). In FLE, the cognitive pathology might be reflected through reductions in coupling between sub networks, which can either be of functional or structural origin or both.

In this study we investigate functional as well as structural whole brain networks in children with FLE. We explore whether abnormalities in graph theoretical measures are present for both the functional and structural networks and correlate these to the cognitive impairment. We hypothesize that differences in whole brain graph theoretical measures can be explained by changes in connectivity between and within large-scale modules. Moreover, the coupling between SC and FC connectivity was compared between children with FLE and healthy controls and correlated with the cognitive impairment.

\section{METHODS}

\section{Participants}

Children with FLE were selected from our reference clinical database and were actively contacted. Inclusion criteria were: a clinically confirmed cryptogenic (i.e., based on EEG and MRI findings, presumed to be symptomatic, but with unknown etiology) localization-related epilepsy with an epileptic focus in the frontal lobe, aged between 8 and 13 years, no other disease that could cause cognitive impairment, and no history of brain injury. Healthy age-matched controls were recruited by advertisements in local newspapers. Inclusion criteria were no history of brain injury or cognitive problems and visiting regular education. All subjects and parents gave 
written informed consent. Approval for the study by the local Medical Ethical Committee was obtained.

\section{Neuropsychological assessment}

Cognitive performance was measured using a computerized visual searching task (CVST) (Aldenkamp et al., 2004). This task consists of finding the right grid pattern that matches the one in the centre of a screen surrounded by 24 other grid patterns. The task is used to assess central information processing speed and perceptual strategies and is considered to be an assessment of frontal lobe function. A detailed description of this task can be found in (Aldenkamp et al., 2004; Vaessen, 2011). By determining the average CVST searching time (reaction time) and the number of correct and incorrect responses during the task, an age-corrected cognitive performance score was generated (i.e. the decile score). After grouping these scores into numbers from 1 (worst score) to 10 (best score), the 3 worst performance scores (1, 2 or 3 ) were considered a manifestation of impaired cognitive performance, while higher scores ( $\geq$ 4) were considered normal.

\section{MRI acquisition}

MRI was performed on a 3.0-Tesla unit equipped with an 8-channel head coil (Philips Achieva, Philips Medical Systems, Best, The Netherlands). Functional MRI data were acquired using a whole-brain single-shot multi-slice echo-planar imaging (EPI) sequence sensitive to the blood-oxygen-level-dependent (BOLD) effect, with TR $2 \mathrm{~s}$, TE $35 \mathrm{~ms}$, flip angle $90^{\circ}$, pixel size $2 \times 2 \mathrm{~mm}^{2}, 32$ contiguous 4-mm thick slices per volume, 195 volumes per acquisition, and an parallel imaging acceleration factor of 1.5 (Sensitivity Encoding).

Diffusion weighted MRI (DWI) was acquired at a pixel size of $2 \times 2 \mathrm{~mm}^{2}$, slice thickness $2 \mathrm{~mm}$, and a $b$-value of $1200 \mathrm{~s} / \mathrm{mm}^{2}$. An echo planar imaging sequence was used with TE $72 \mathrm{~ms}$, TR $6584 \mathrm{~ms}$, and parallel imaging acceleration factor of 2. A set of 61 gradient directions was used, optimized via electrostatic repulsion to ensure homogenous distribution over the sphere (Jones, Horsfield et al. 1999). In addition, a single non-diffusion weighted scan (b0-scan) was obtained. The DWI acquisition time was 8 minutes.

For anatomic reference, a T1-weigthed 3D spoiled fast gradient echo pulse sequence was acquired with the following parameters: TR $8.1 \mathrm{~ms}$, TE $3.7 \mathrm{~ms}$, flip angle $8^{\circ}$, field of view (FOV) $256 \times 256 \times 180 \mathrm{~mm}^{3}$, and voxel size $1 \times 1 \times 1 \mathrm{~mm}^{3}$.

\section{Inclusion}

Subjects were excluded when head movements exceeded $1.5 \mathrm{~mm} / \mathrm{s}$ or 1.5 degrees $/ \mathrm{s}$ in at least one direction. Data of nine patients were excluded from further analysis because of movement related artifacts ( $n=6 ; 2$ controls, 4 patients), EPI artifacts ( $n=3$; 
2 controls, 1 patient). From the DWI data, five subjects were excluded from further analysis due to EPI artifacts ( $n=1$ controls, $n=4$ patients). For analysis the final study population consisted of 26 patients and 36 healthy controls.

Six patients did not complete the neuropsychological assessment and thus had no CVST scores. These patients are included in the group analysis, but not in the correlation analysis. In total, 9 FLE patients had a decile score below 4 and were considered cognitively impaired. Mean CVST reaction time was significantly higher in the patient group compared to the healthy control group (controls: $7.5 \pm 6.4 \mathrm{~s}$ (mean $\pm S D)$, patients: $23.6 \pm 9.9 \mathrm{~s}, p<0.006)$.

\section{Network construction}

\section{Anatomical parcellation}

Freesurfer (Martinos Center of Biomedical Imaging, Boston, US) software was used to segment the T1 images of each subject into cortical and subcortical regions. Freesurfer uses a surface based alignment procedure, which might be more accurate than a volume based alignment of a cortical atlas (Ghosh et al., 2010).

The Freesurfer cortical regions were further refined into a larger number of smaller regions. We started by dividing each region from the standard Freesurfer template into two more or less equally sized regions by principal component analysis. This segmentation was performed in the spherical surface coordinates. The cortical surface of each hemisphere can be modeled as the surface of a sphere; each point on the cortical surface can be related to a point on the sphere which is defined by its longitude and latitude. The first principal component (a 2D vector), together with the center of gravity of the cortex point within the region (a 2D point), defines a line in 2D space which divides the region into two sub regions according to the maximum spatial variance of the region (e.g. a "stretched" region will be divided along its main longitudinal axis). Regions were subsequently subdivided with the criterion that a division must not yield a sub region with a size smaller than 1200 cortical points. The final result was a parcellation of 95 regions with comparable sizes (of at least 1200 cortex points) per hemisphere. The regions were converted from the spherical format to the cortex of each individual by standard Freesurfer routines. The subcortical regions were used in their original Freesurfer format.

The cortical and subcortical parcellation of each individual in native T1 space was transformed to the native DWI or fMRI space by applying a rigid body transformation. The transformations from Freesurfer standard space to T1 space and from T1 space to DWI or fMRI space can result in the loss of several regions by partial volume effects. Therefore, only regions that were present in all the parcellations in DWI and FMRI space were used. This resulted in a parcellation with 205 bi-lateral regions for all subjects ( $2 \times 95$ cortical regions and 15 subcortical regions). 


\section{Functional network construction}

The BOLD images were corrected for motion artifacts using SPM5 (Wellcome Trust Centre for Neuroimaging, UCL, London, UK) software. The images were then high-pass filtered with a $\sigma$ of 25 scans ( 50 seconds) and spatially smoothed $(\sigma=1.7 \mathrm{~mm}$ ) using FSL 4.1.7 (Oxford University, Oxford, UK) software. Subsequently, the CSF and whole brain signal time course were removed from the images using standard linear regression. The resulting residual time series were used for further analysis. Lastly, the images were low-pass filtered ( $\sigma=2 \mathrm{~s}$, i.e. 1 dynamic scan interval) to remove the detrimental effects of high-frequency noise components. Using Matlab (The MathWorks Inc., Natick, US; version 7.6.0), the Pearson's linear correlation coefficient was calculated between the region-averaged time-series of all pairs of Freesurfer regions. In this way, a $205 \times 205$ connectivity matrix was calculated for each subject.

\section{Structural network construction}

Each data set was spatially co-registered to the $b=0$ image with an affine transformation to correct for head motion and eddy-current distortions utilizing CATNAP (Co-registration, Adjustment, and Tensor-solving, a Nicely Automated Program, version 1.3) software (Farrell et al., 2007). The set of gradient vectors was adjusted according to the rotation of the individual images.

All DWI analyses, the tractography and tract segmentations were performed using the MRtrix software package (Tournier et al., 2007). Diffusion tensor (DT) fits were performed to calculate FA and ADC maps. In addition, fiber orientation distributions (FODs), representing local fiber orientation, were estimated using constrained spherical deconvolution (CSD). In CSD, the diffusion profile is converted to the underlying fiber orientations by a constrained deconvolution method (Tournier et al., 2007). The CSD response function was estimated from data with high FA voxels values (FA>0.7). Slice drop-outs are a common phenomenon in DW-EPI, especially in the presence of motion (Rohde et al., 2004). To reduce the effects of corrupted slices on FOD estimation, a method was developed in which corrupted slices were automatically detected and removed from the data. Subsequently, FOD's were estimated per slice with a slice-specific gradient set (i.e. without the directions corresponding to the removed slices). On average 46 (out of 3660. i.e. approx. 1\%) slices were corrupted per subject. The number of corrupted slices did not differ between the groups.

Within the white matter, five million evenly distributed seeds were placed and a streamline was started from each seed. Subsequently, for each pair of regions from the anatomical atlas, the subset of tracts connecting these two regions were identified from the set of tracts of the whole brain tractogram. Connection weights were determined by calculating the tract volume of the voxels traversed by the streamlines of the connection, divided by the total intracranial volume (Vaessen et al., 
2011). As an additional noise filter, voxels that were traversed by fewer than 2 tracts were eliminated from the analysis.

\section{Network characteristics}

For each subject, values of network measures were calculated from the individual SC or FC matrices. We included 3 network measures: characteristic path length $(L)$, clustering coefficient $(C)$, and modularity, using algorithms implemented in the Brain Connectivity Toolbox (Rubinov and Sporns, 2010). A detailed description of path length and clustering can be found elsewhere (Rubinov and Sporns, 2010). Network measures were assessed over a range of sparsity values (Vlooswijk et al., 2011). Modularity quantifies the degree to which a brain network is organized in isolated sub networks (i.e. the modules). The more isolated the sub networks are, the higher the modularity. We used algorithm developed by Newman et al. (Newman, 2006) to quantify the modularity of the brain. With this algorithm the brain was automatically subdivided into a number of modules (i.e. groups of connected nodes) with maximal connection strength within and minimal connection strength between the modules, creating a so-called "optimal community structure" (OCS) of the brain. To avoid effects of differently organized modules in patients and controls, the within- and between-module connectivity was determined from the connectivity matrix that comprised the mean of entire study population, thus the combination of patients and controls.

\section{Analysis of within- and between-module connectivity}

To assess the potential differences in within- and between-module connectivity, the modular organization of the FC was calculated, by applying the modularity algorithm to the FC matrix averaged over all subjects. Next, each edge was classified as either between-module (the edge connects nodes of two different modules) or withinmodule (the edge connects nodes of the same module). Connection strengths of the within- and between-module edges from the FC and SC modules were averaged. The within-module connections were assessed both as the aggregate over all modules and for each module separately. The weak and negative edges of the FC matrices might contain relevant information on between-module connectivity, therefore the unthresholded FC and SC matrices were used.

\section{Statistical analysis}

Between-group effects in network measures and connection strengths were assessed by two-sample Student's $t$-tests. We compared the entire patient group (EP) and the cognitively impaired patient group (IP) with the healthy control group. Pearson's (linear) correlation coefficients $(r)$ were calculated between cognitive performance (CVST reaction time), age, connection strengths and network measures. This analysis was performed for the EP group and control group, separately. After the coupling 
between the FC and SC edge strengths was calculated by a correlation analysis for each subject, the individual FC-SC coupling values were associated with CVST scores and age.

\section{RESULTS}

\section{Network connectivity}

\section{Functional connectivity}

The mean functional connectivity value (i.e. Fisher-z transformed time series correlations) over all connections did not significantly differ between the EP, IP and healthy control groups. Mean FC was not significantly correlated with age or with CVST scores.

\section{Structural connectivity}

For the structural connectivity (i.e. relative tract volume over all connections) the IP group $\left(9.5 \cdot 10^{-4} \pm 0.5 \cdot 10^{-4}, p<0.07\right)$, but not the EP group $\left(8.9 \cdot 10^{-4} \pm 0.4 \cdot 10^{-4}\right.$, n.s. $)$, showed a trend of higher mean connectivity values than the control group $\left(8.5 \cdot 10^{-4} \pm 0.1 \cdot 10^{-4}\right)$. Mean structural connectivity was not correlated with CVST score or age. Mean FA was not different between the groups, but increased with age in both the control $(r=0.32$, $p<0.05)$ and EP group $(r=0.44, p<0.02)$. Mean ADC was also not different between the groups and did not significantly correlate with age in the control group, while a negative correlation was found in the EP group $(r=-0.47, p<0.01)$.

\section{Network topology}

\section{Functional connectivity}

The cluster coefficient was significantly higher for both the EP and IP groups compared to the healthy control group over the entire sparsity range (0.55-0.90). The path length was significantly higher for the EP and IP groups compared to the control group over the sparsity range $0.55-0.80$. The modularity was significantly higher for the IP group compared to the control group over the entire sparsity range, while the EP group had a significantly higher modularity over the sparsity range $0.55-0.80$ (Figure. 6.1A).

The cluster coefficient, path length, nor modularity of the functional networks was significantly correlated with CVST score or age for the control or EP group. 


\section{Structural connectivity}

Although the means of the cluster coefficient, path length, and modularity showed slightly higher values for the IP group compared to the control and EP groups, none of these differences were significant. The EP group also did not display significant differences compared to the control group (Figure 6.1B).

In the EP group there was a negative correlation trend between $C$ and age (mean $r$ over entire sparsity range $r=-0.37$, mean $p=0.06$ ) and negative correlation between modularity and age (mean $r$ over entire sparsity range $r=-0.40$, mean $p=0.04$ ). In the control group, path length showed a trend for positive correlation with CVST scores (mean $r$ over entire sparsity range $=0.29$, mean $p=0.09$ ). Modularity scores increased with CVST scores in the EP group (mean $r$ over entire sparsity range $r=0.51$, mean $p=0.02$ ).

\section{(A) Functional connectivity}
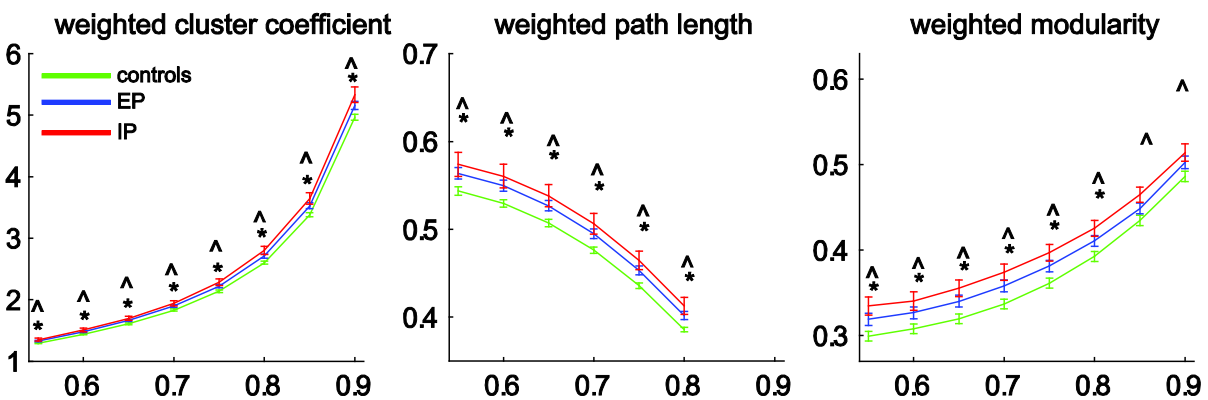

(B) Structural connectivity
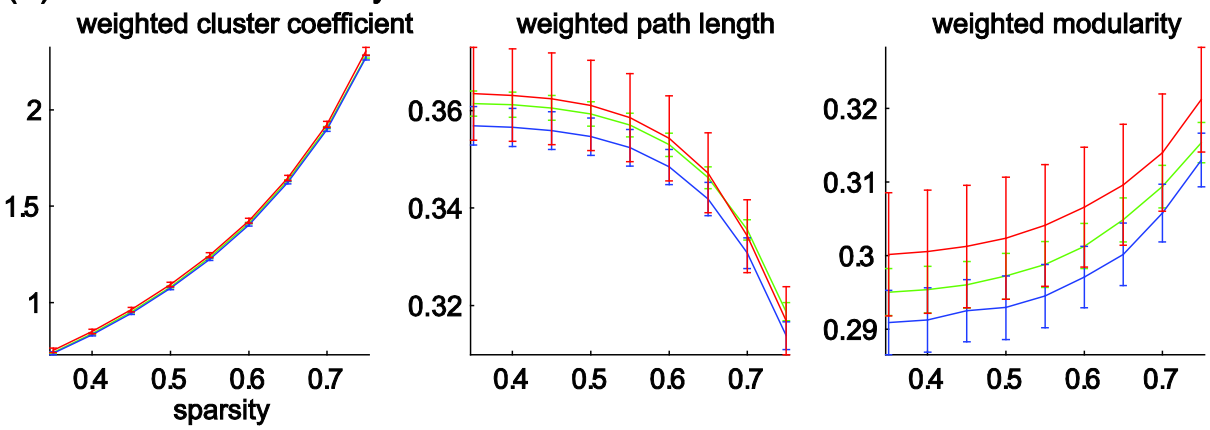

Figure 6.1 Network metrics for functional (A) and structural (B) connectivity as a function of sparsity. The networks measures for the control group (green), the entire patient group (EP, blue) and the impaired patient group (IP, red) as a function of sparsity. Symbols for statistical comparison: $*: p<0.05$ for EP versus control group, ^: $p<0.05$ for IP versus control group. 


\section{Modular organization}

The modularity algorithm determined four modules from the averaged FC matrix over all subjects (Figure 6.2A). The spatial organization of module 1 (blue) highly resembled to the default mode network (DMN) (Greicius et al., 2003), with regions in the frontal, temporal and parietal lobes. The second module (red) consisted of frontal and subcortical regions. The relatively small third module (yellow) was centered in the occipital lobe. Module 4 (green) was distributed over frontal, temporal and occipital regions. All modules were highly symmetric with respect to the interhemispheric fissure. After structural connections were ordered similar to the organization of the FC modularity matrix, the structural organization of the modules revealed more or less bilateral structural sub networks (Figure 6.2A and 6.2B).

For the SC matrix the modularity algorithm determined only two modules, which were separated between the two hemispheres. After functional connections were ordered according to these $\mathrm{SC}$ modules, no further sub organization became evident (Figure $6.2 \mathrm{C}$ and $6.2 \mathrm{D})$.

\section{Modular connectivity}

FC (Fisher-z transformed correlation values) and SC (relative tract volumes) values were classified as between-module, within-module averaged over all modules (i.e. aggregated within-module) and individual within-module connections. These connection values are listed per group in Table 6.1.

\section{Between-module connectivity}

The between-module FC was lower in the IP group $(p<0.017)$ and the EP group $(p<0.06)$ compared to the control group. A trend for higher between-module SC was found in the IP group compared to the healthy control group $(p=0.07)$, while no differences were found between the EP and control group (Figure 6.3C). No significant correlations were found between CVST score or age and FC or SC between-module connectivity.

\section{Aggregated within-module connectivity}

A trend for higher within-module FC values was observed for the IP group $(p=0.095)$ and the EP group $(p=0.08)$ compared to the control group. The SC within-module connection strengths displayed a trend for higher values in the IP group $(p=0.08)$ compared to the control group, but not for the EP group (Figure 6.3D). No significant correlations were found between CVST score or age and within-module FC or SC connectivity. 
(A) FC sorted by FC modularity

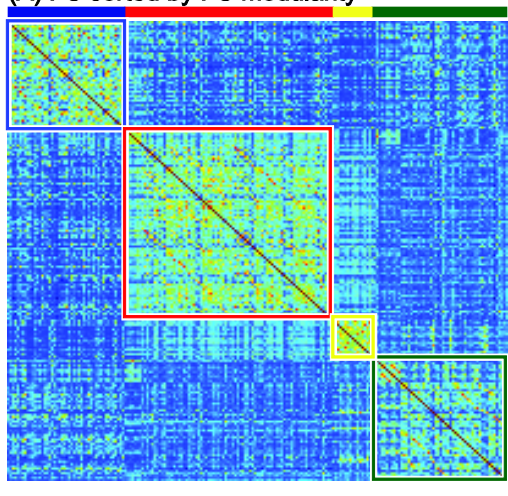

(C) FC sorted by SC modularity

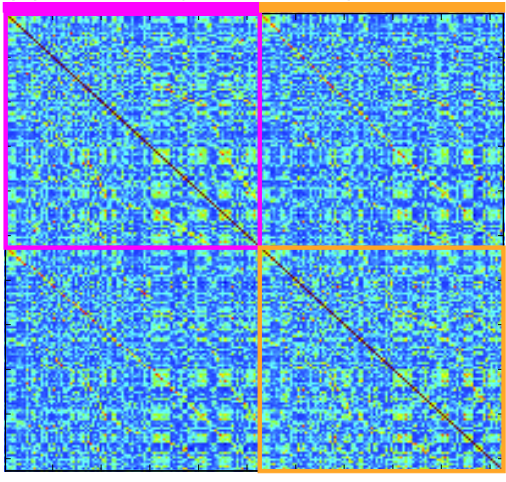

\section{(B) SC sorted by FC modularity}
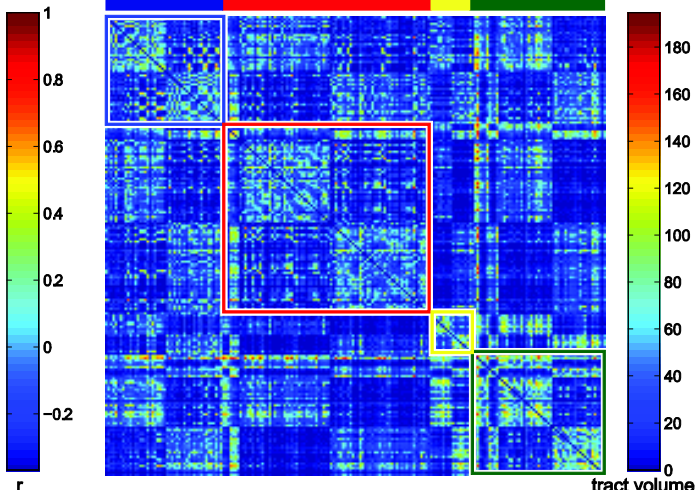

(D) SC sorted by SC modularity

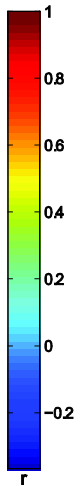

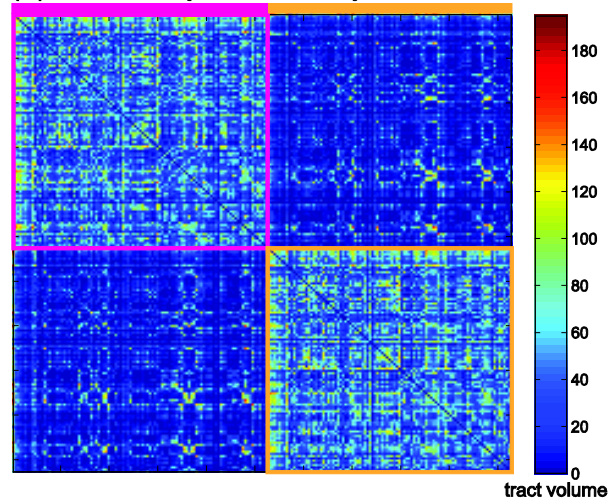

Figure 6.2 Group average connection matrices sorted by module. (A) Functional connectivity. Colored rectangles indicate the modules. High within-module connectivity is clearly visible by the higher values (more hot colors), while between-module connectivity is more sparse (more cold colors). (B) Structural connectivity sorted by functional modules. The functional modules are organized bi-laterally, while the SC has strong inter-hemispheric connectivity and low intra-hemispheric connectivity clearly visible in the block patterns. (C) The FC matrix sorted by the modular organization derived from the SC. The two found SC modules are basically the left and right hemisphere. From the FC it is visible that strong inter-hemispheric connections are present within the two modules. (D) The SC sorted by SC modularity. Strong intrahemispheric connections are visible, while inter-hemispheric connections (and thus betweenmodule connections) are weaker.

\section{Separate within-module connectivity}

For FC, the strongest differences in within-modularity between patients and controls were found for module 4. Module 4 showed higher within module FC for the EP group $(p=0.012)$, while a trend was observed for the IP group $(p=0.071)$, compared to the control group (Figure 6.3E). A positive association between CVST score (higher scores indicate reduced cognitive performance) and within module FC was found for module 4 in the control group $(r=0.36, p<0.04)$. The other modules did not reveal associations 
between CVST score and FC in neither the control group nor the EP group. Age was not significantly correlated with any of the within module FC values.

The within-module SC of module 4 was also significantly higher for the IP group $(p=0.013)$ compared to the control group. The other three modules did not show significant group differences in SC (Figure 6.3F). For module 4 the within-module SC increased with higher CVST score (worse cognitive performance) in the EP group $(r=0.55, p<0.01)$. No association between SC and CVST score was found in any of the other modules. Age was not significantly correlated with any of the within-module SC values.

\begin{tabular}{llllll}
\hline & $\begin{array}{l}\text { Controls } \\
\text { (mean } \pm \text { SEM) }\end{array}$ & $\begin{array}{l}\text { EP } \\
\text { (mean } \pm \text { SEM) }\end{array}$ & $\begin{array}{l}\text {-value } \\
(C-E P)\end{array}$ & $\begin{array}{l}\text { IP } \\
\text { (mean } \pm S E M)\end{array}$ & $\begin{array}{l}\text { p-value } \\
(C-I P)\end{array}$ \\
\hline FC & & & & \\
BT & $-0.026 \pm 0.003$ & $-0.033 \pm 0.004$ & 0.06 & $-0.041 \pm 0.005$ & 0.02 \\
WI & $0.245 \pm 0.005$ & $0.260 \pm 0.008$ & 0.08 & $0.265 \pm 0.011$ & 0.10 \\
WI1 & $0.284 \pm 0.021$ & $0.299 \pm 0.010$ & n.s. & $0.313 \pm 0.024$ & n.s. \\
WI2 & $0.244 \pm 0.005$ & $0.255 \pm 0.014$ & n.s. & $0.258 \pm 0.013$ & n.s. \\
WI3 & $0.606 \pm 0.007$ & $0.643 \pm 0.029$ & n.s. & $0.700 \pm 0.057$ & 0.07 \\
WI4 & $0.176 \pm 0.005$ & $0.197 \pm 0.009$ & 0.01 & $0.196 \pm 0.009$ & 0.07 \\
SC $\left(* 10^{-4}\right)$ & & & & & \\
BT & $7.8 \pm 0.1$ & $14.9 \pm 0.1$ & n.s. & $8.3 \pm 0.1$ & 0.07 \\
WI & $9.9 \pm 0.2$ & $10.2 \pm 0.2$ & n.s. & $11.0 \pm 0.4$ & 0.08 \\
WI1 & $9.70 \pm 0.2$ & $10.0 \pm 0.3$ & n.s. & $11.0 \pm 0.6$ & n.s. \\
WI2 & $8.0 \pm 0.2$ & $8.2 \pm 0.2$ & n.s. & $7.9 \pm 0.3$ & n.s. \\
WI3 & $15.0 \pm 0.4$ & $14.7 \pm 0.4$ & n.s. & $16.0 \pm 0.8$ & n.s. \\
WI4 & $13.0 \pm 0.3$ & $14.0 \pm 0.5$ & n.s. & $15.0 \pm 0.1$ & 0.01 \\
\hline
\end{tabular}

Table 6.1 The modularity measures derived from the functional connectivity (FC) and structural connectivity (SC) matrices of the entire patient (EP) group, the cognitively impaired patient (IP) group, and the healthy controls. The modularity measures comprise the between-module (BT) and within module connectivity values; the latter averaged over all four functional modules (WI) and per functional module (WI1...4).

\section{Structure-function correlation}

The EP and IP groups did not differ significantly from the control group in SC-FC coupling. CVST score was not significantly associated with FC-SC coupling. A trend for a negative association was found in the EP group between FC-SC coupling and age ( $r=-$ $0.38, p=0.06)$, while for the control group a significant positive association $(r=0.40$, $p<0.01$ ) was found (Figure 6.4). 
A

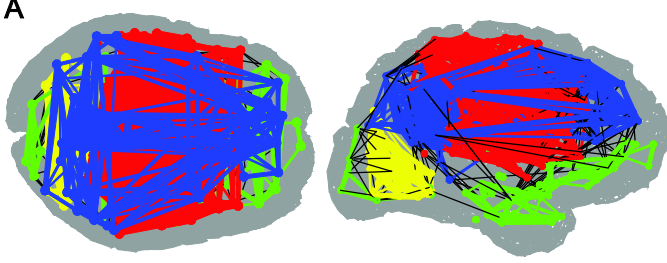

B

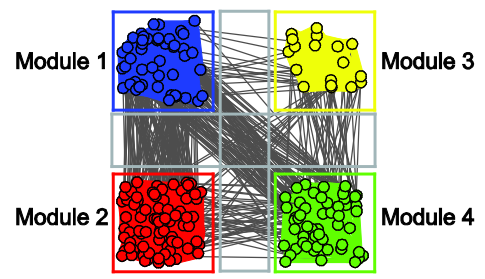

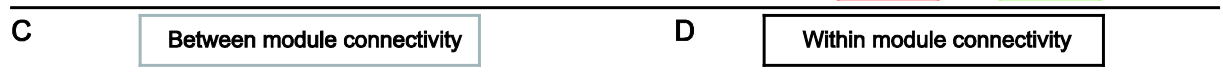
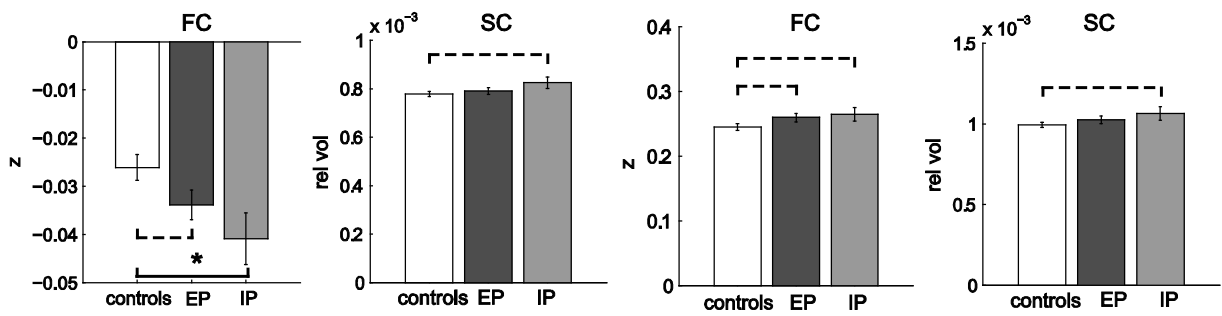

\section{E FC seperate modules}
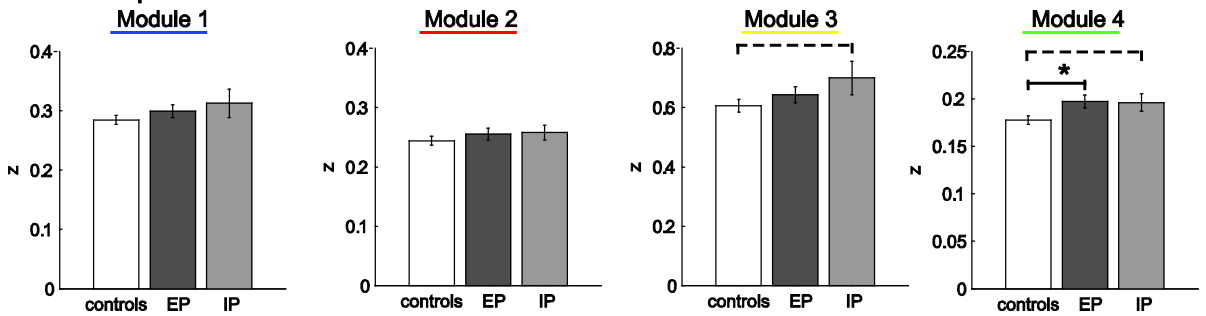

F SC seperate modules
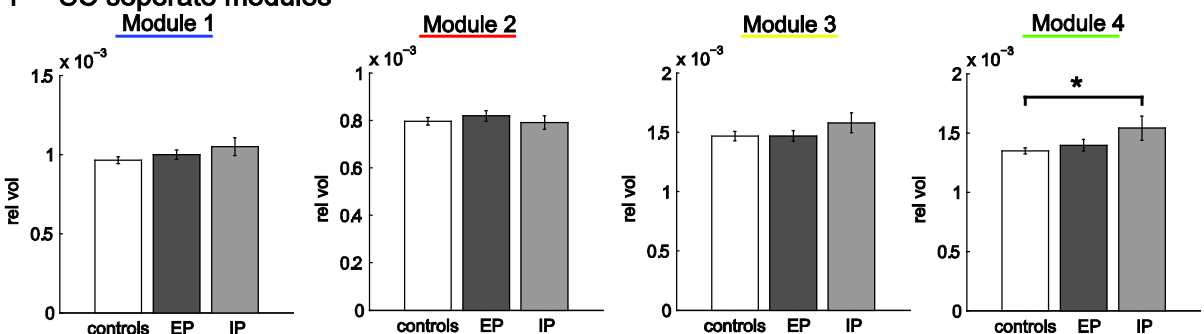

Figure 6.3 Within- and between-module connectivity for FC and SC. (A) Modular organization of FC. Within-module connections are colored as in Figure 6.2A. (B) An alternative presentation of the modular organization. The nodes of each separate module are depicted spatially segregated. The gray lines indicate the between-module connections. (C) All between-module connection strengths (gray lines in B) for FC and SC were averaged and compared between the different groups. (D) The within-module connections over all four modules were also averaged for the different groups and compared. (E) FC mean within-module connection strengths are compared between the groups for the four different modules. (F) Withinmodule SC. Bars display the mean \pm SEM. 


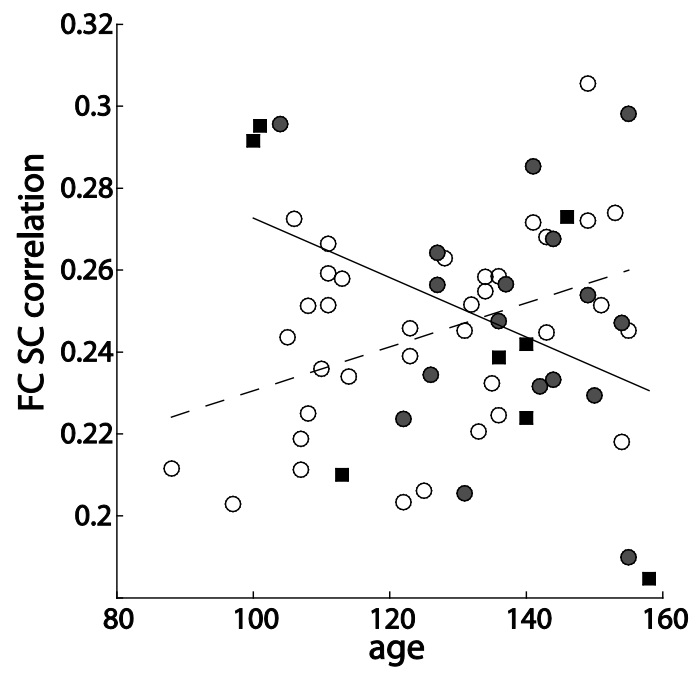

Figure 6.4 Function structure correlation with age. Coupling of SC and FC versus age of the control group (open circles) and unimpaired patient group (grey circles) and impaired patients (black squares). Each dot represents the correlation of all non-zero edge strengths of the FC and SC for that person. Regression lines for the control group (dashed) and entire patient group (solid) are shown. Note the increasing FC-SC correlation with age for the healthy controls, which was reversed for the patients with FLE.

\section{DISCUSSION}

In this study we compared the FC and SC of cerebral networks in children with FLE to healthy controls and investigated whether the associated cognitive impairment in FLE is reflected by an aberrant functional or structural modular organization. For the whole brain network the functional network clustering, path length and modularity appeared more sensitive than structural network measures to discern children with FLE, and particularly those with cognitive impairment from healthy controls. To focus on the organization of sub networks, modularity analysis resulted in a division of the whole brain network into four large-scale functional modules with relatively strong within-module and relatively weak between-module connections. The functional modular organization in childhood FLE appeared to be aberrant in the sense that between-module connectivity was weakest in the children with FLE who had cognitive impairments. In more detail it was found that module 4, which comprised large parts of the frontal and temporal lobe, exhibited both increased functional and structural within-module connectivity relative to controls. For this particular module 4 the normal negative correlation between within-module FC and cognitive performance, as observed in healthy controls, was lost in the FLE patients. Although, in this module, an increase in SC with decreased cognitive performance was observed for the FLE patients, but not for the controls. 


\section{Global functional and structural network abnormalities}

Whole brain functional network organization appeared to be disturbed in children with FLE, while the global structural network organization did not show such salient effects. Although structural network organization was not different in FLE patients compared to controls, stronger structural modularity was associated with worse cognitive scores in the patients. The deviant whole brain functional network measures were most salient in the cognitively impaired patients, and suggest that the functional network organization is linked to the cognitive pathology. Furthermore, the functional but not the structural abnormalities in network organization could imply that functional disturbances precede structural abnormalities in childhood FLE. Conversely, differences in sensitivity of the different imaging modalities in detecting abnormalities could also underlie these results.

\section{Aberrant functional and structural modules}

The stronger modular organization in children FLE with cognitive impairment supports the hypothesis that whole brain connectional abnormalities can be traced back to differences in connectivity between and within more or less isolated functional modules. This is in agreement with a recent study where a loss of communication between functional modules was observed in Alzheimer Disease patients (de Haan et al., 2011). Between-module functional connectivity was decreased in the impaired children, while at the same time an increase in structural connectivity was observed. For both the functional and structural networks, an increase in within-module connectivity was found. Hence, children with FLE and cognitive impairments had an overall increase in structural connectivity, while the differences in functional connectivity were characterized by decreased between- and increased within-module connectivity.

Most striking for the relation between cognitive impairment and modular organization was the aberrant connectivity of module 4, which covers large parts of the frontal, temporal lobe. For this module, both the functional and structural within-module connectivity was increased, specifically for the cognitively impaired children, relative to the healthy controls. Considering the decline in higher cognitive functions for children with FLE, this observation seems to hint in more detail at the neuropathological substrate of cognitive impairment in childhood FLE.

\section{Developmental effect on connectivity}

We found that the coupling between FC and SC increased with age in the healthy controls, which is in agreement with (Hagmann et al., 2010). However, the increase was not found for the children with FLE. This could indicate that the normal development in SC-FC relation is disturbed in childhood FLE. It is currently unclear to what extend $\mathrm{FC}$ and SC modularity differ and how this relation depends on age. 
Some developmental processes such as an increase in white matter integrity or functional correlation with age might manifest in a distributed whole brain wide manner and thus influence the mean SC or FC. Since the connectivity matrices were corrected for mean connectivity values, such effect might not be evident in the topological measures $(L$ and $C$ ). Therefore, we also investigated the underlying data for the mean connectivity values. For the mean SC, an increase in FA and a decrease in $A D C$ were observed with increasing age in the entire patient group, while FC was not correlated with age. Changes in FA and ADC with age have extensively been reported in literature (Stadlbauer et al., 2008; Hagmann et al., 2010; Westlye et al., 2010) and are a sign of normal development. We could not detect significant group differences in mean FA or mean ADC, but a trend for larger relative tract volumes (thus SC values) in the impaired patient group was found. Given the narrow age range ( $8-13$ year) of the subjects in this study for which correlations with age were found, studies with larger age ranges or preferably longitudinal studies are needed to infer on the expected abnormal developmental trajectories in childhood FLE.

\section{Conclusion}

In children with FLE it was shown that the more isolated functional brain sub networks appear to function the more cognitively impaired these children are. This observation can be interpreted in a way that cognitively impaired patients have a less efficient interregional transfer of information between functional sub networks. As this effect was not directly evident from structural connectivity measures, this raises the question whether functional changes precede structural changes. Future studies with patients in a broader age range might clarify the relation between structural and functional abnormalities in relation to cognitive developmental abnormalities in more detail. 


\section{REFERENCES}

Aldenkamp AP, Ar ends J, Verspeek S, Berting M (2004) The cognitive impact of epileptiform EEG-discharges; relationship with type of cognitive task. Child Neuropsychol 10:297-305.

Berg AT (2011) Epilepsy, cognition, and behavior: The clinical picture. Epilepsia 52 Suppl 1:7-12.

Bettus G, Guedj E, Joyeux F, Confort-Gouny S, Soulier E, Laguitton V, Cozzone PJ, Chauvel P, Ranjeva JP, Bartolomei F, Guye M (2009) Decreased basal fMRI functional connectivity in epileptogenic networks and contralateral compensatory mechanisms. Hum Brain Mapp 30:1580-1591.

Bullmore E, Sporns O (2009) Complex brain networks: graph theoretical analysis of structural and functional systems. Nat Rev Neurosci 10:186-198.

de Haan W, van der Flier WM, Koene T, Smits LL, Scheltens P, Stam CJ (2011) Disrupted modular brain dynamics reflect cognitive dysfunction in Alzheimer's disease. Neuroimage.

Ethofer T, Gschwind M, Vuilleumier P (2011) Processing social aspects of human gaze: a combined fMRI-DTI study. Neuroimage 55:411-419.

Farrell JA, Landman BA, Jones CK, Smith SA, Prince JL, van ZijI PC, Mori S (2007) Effects of signal-to-noise ratio on the accuracy and reproducibility of diffusion tensor imaging-derived fractional anisotropy, mean diffusivity, and principal eigenvector measurements at $1.5 \mathrm{~T}$. Journal of magnetic resonance imaging : JMRI 26:756-767.

Fox MD, Raichle ME (2007) Spontaneous fluctuations in brain activity observed with functional magnetic resonance imaging. Nat Rev Neurosci 8:700-711.

Friston KJ (1994) Functional and effective connectivity in neuroimaging: A synthesis. Human Brain Mapping 2:56-78.

Ghosh SS, Kakunoori S, Augustinack J, Nieto-Castanon A, Kovelman I, Gaab N, Christodoulou JA, Triantafyllou C, Gabrieli JD, Fischl B (2010) Evaluating the validity of volume-based and surfacebased brain image registration for developmental cognitive neuroscience studies in children 4 to 11 years of age. Neuroimage 53:85-93.

Greicius MD, Krasnow B, Reiss AL, Menon V (2003) Functional connectivity in the resting brain: a network analysis of the default mode hypothesis. Proceedings of the National Academy of Sciences of the United States of America 100:253-258.

Greicius MD, Supekar K, Menon V, Dougherty RF (2009) Resting-state functional connectivity reflects structural connectivity in the default mode network. Cerebral cortex 19:72-78.

Hagmann P, Sporns O, Madan N, Cammoun L, Pienaar R, Wedeen VJ, Meuli R, Thiran JP, Grant PE (2010) White matter maturation reshapes structural connectivity in the late developing human brain. Proc Natl Acad Sci U S A 107:19067-19072.

Honey CJ, Thivierge JP, Sporns O (2010) Can structure predict function in the human brain? Neuroimage 52:766-776

Honey CJ, Sporns O, Cammoun L, Gigandet X, Thiran JP, Meuli R, Hagmann P (2009) Predicting human resting-state functional connectivity from structural connectivity. Proceedings of the National Academy of Sciences of the United States of America 106:2035-2040.

Johansen-Berg H (2011) The future of functionally-related structural change assessment. Neuroimage.

Liao W, Zhang Z, Pan Z, Mantini D, Ding J, Duan X, Luo C, Lu G, Chen H (2010) Alter ed functional connectivity and small-world in mesial temporal lobe epilepsy. PLoS One 5:e8525.

Manford M, Hart YM, Sander JW, Shorvon SD (1992) National General Practice Study of Epilepsy (NGPSE): partial seizure patterns in a general population. Neurology 42:1911-1917. 
Newman ME (2006) Modularity and community structure in networks. Proc Natl Acad Sci U S A 103:85778582.

Pereira FR, Alessio A, Sercheli MS, Pedro T, Bilevicius E, Rondina JM, Ozelo HF, Castellano G, Covolan RJ, Damasceno BP, Cendes F (2010) Asymmetrical hippocampal connectivity in mesial temporal lobe epilepsy: evidence from resting state fMRI. BMC Neurosci 11:66.

Power JD, Fair DA, Schlaggar BL, Petersen SE (2010) The development of human functional brain networks. Neuron 67:735-748.

Pravata E, Sestieri C, Mantini D, Briganti C, Colicchio G, Marra C, Colosimo C, Tartaro A, Romani GL, Caulo M (2011) Functional connectivity MR imaging of the language network in patients with drugresistant epilepsy. AJNR Am J Neuroradiol 32:532-540.

Rohde GK, Barnett AS, Basser PJ, Marenco S, Pierpaoli C (2004) Comprehensive approach for correction of motion and distortion in diffusion-weighted MRI. Magnetic resonance in medicine : official journal of the Society of Magnetic Resonance in Medicine / Society of Magnetic Resonance in Medicine 51:103-114.

Rubinov M, Sporns O (2010) Complex network measures of brain connectivity: uses and interpretations. Neuroimage 52:1059-1069.

Skudlarski P, Jagannathan K, Anderson K, Stevens MC, Calhoun VD, Skudlarska BA, Pearlson G (2010) Brain connectivity is not only lower but different in schizophrenia: a combined anatomical and functional approach. Biological psychiatry 68:61-69.

Sporns O (2011) The human connectome: a complex network. Annals of the New York Academy of Sciences 1224:109-125.

Stadlbauer A, Salomonowitz E, Strunk G, Hammen T, Ganslandt O (2008) Age-related degradation in the central nervous system: assessment with diffusion-tensor imaging and quantitative fiber tracking. Radiology 247:179-188.

Stam CJ, Reijneveld JC (2007) Graph theoretical analysis of complex networks in the brain. Nonlinear Biomed Phys 1:3.

Tournier JD, Calamante F, Connelly A (2007) Robust determination of the fibre orientation distribution in diffusion MRI: non-negativity constrained super-resolved spherical deconvolution. Neuroimage 35:1459-1472.

Tournier JD, Mori S, Leemans A (2011) Diffusion tensor imaging and beyond. Magnetic resonance in medicine : official journal of the Society of Magnetic Resonance in Medicine / Society of Magnetic Resonance in Medicine 65:1532-1556.

Vaessen MJ (2011) Abnormal modular organization of cerebral functional networks in cognitively impaired children with frontal lobe epilepsy. in preparation.

Vaessen MJ, Jansen JF, Vlooswijk MC, Hofman PA, Majoie HJ, Aldenkamp AP, Backes WH (2011) White Matter Network Abnormalities Are Associated with Cognitive Decline in Chronic Epilepsy. Cerebral cortex.

Van den Heuvel MP, Hulshoff Pol HE (2010) Exploring the brain network: a review on resting-state fMRI functional connectivity. Eur Neuropsychopharmacol 20:519-534.

van den Heuvel MP, Stam CJ, Kahn RS, Hulshoff Pol HE (2009) Efficiency of functional brain networks and intellectual performance. J Neurosci 29:7619-7624.

Vlooswijk MC, Jansen JF, Majoie HJ, Hofman PA, de Krom MC, Aldenkamp AP, Backes WH (2010) Functional connectivity and language impairment in cryptogenic localization-related epilepsy. Neurology 75:395-402. 
Vlooswijk MC, Vaessen MJ, Jansen JF, de Krom MC, Majoie HJ, Hofman PA, Aldenkamp AP, Backes WH (2011) Loss of network efficiency associated with cognitive decline in chronic epilepsy. Neurology 77:938-944.

Waites AB, Briellmann RS, Saling MM, Abbott DF, Jackson GD (2006) Functional connectivity networks are disrupted in left temporal lobe epilepsy. Ann Neurol 59:335-343.

Westlye LT, Walhovd KB, Dale AM, Bjornerud A, Due-Tonnessen P, Engvig A, Grydeland H, Tamnes CK, Ostby $Y$, Fjell AM (2010) Life-span changes of the human brain white matter: diffusion tensor imaging (DTI) and volumetry. Cerebral cortex 20:2055-2068.

Zhang Z, Liao W, Chen H, Mantini D, Ding JR, Xu Q, Wang Z, Yuan C, Chen G, Jiao Q, Lu G (2011) Altered functional-structural coupling of large-scale brain networks in idiopathic generalized epilepsy. Brain : a journal of neurology 134:2912-2928. 
CHAPTER

General discussion 
144 Chapter 7 
The general aim of the research described in this thesis was to develop and explore the use of graph analysis methods to find the neuronal correlate of cognitive impairment in epilepsy. This was pursued by deriving graph theoretical parameters from whole brain networks constructed from functional and diffusion weighted MRI data. Research to date has mainly focused on the "focal" approach: relating cognitive parameters to localized functional and macro- and micro-structural tissue abnormalities (Berl et al., 2005; Cheung et al., 2006; Weber et al., 2006; Powell et al., 2007; Focke et al., 2008; Yogarajah et al., 2008; Bonelli et al., 2010; Meng et al., 2010; Riley et al., 2010). Although this approach is viable, in this thesis we advocated for a different paradigm where the brain is viewed as a highly interconnected system and analysis methods should be tailored towards relating cognitive deficits to characteristics of large-scale networks. In the following paragraphs our findings and interpretations using this approach will be discussed in a broader perspective. In addition, the scientific and clinical implications of our main findings will be addressed, some methodological issues will be considered, and recommendations for future research will be given.

\section{Summary of findings}

Abnormalities in large scale organization of brain networks appear to underlie cognitive problems in patients with chronic epilepsy. In chapter 3 it was shown that reduced efficiency of cerebral functional networks was evident in adult patients with chronic cryptogenic epilepsy and that the cerebral efficiency was related to measures of global cognitive performance. Chapter 4 concerns the same study population, but here white matter networks were investigated with fiber tractography. Differences in graph metrics between patients with cognitive impairment and healthy controls were identified, as well as a strong relation between cognitive scores and graph metrics. A cohort of children with frontal lobe epilepsy was investigated in chapter 5 . Functional brain networks showed connectional abnormalities that were mainly expressed as a reduction in functional coupling between sub-networks (modules) which was correlated with reduced cognitive scores. In chapter 6 it was studied whether the functional abnormalities observed in children with FLE could also be related to white matter networks. A reduction in functional coupling between networks was identified in the patient group in addition to increased modularity, clustering and path length. However, an overall increase in white matter (structural) connectivity was observed, although whole brain white matter graph metrics did not show any differences. None of these studies revealed a salient relation between clinical factors and cognitive performance. Therefore, the added value of imaging was evident in chapter 3 , where a statistical relation was found between drug load and intelligence when imaging parameters of network efficiency were introduced as a mediating variable. 


\section{Brain networks and cognition in adults and children with epilepsy}

Abnormalities in brain network organization were found in both adults and children with cryptogenic epilepsy. What are the similarities and differences observed, and how can we interpret the direction of change in network parameters? Adult patients, especially those with cognitive impairment, had higher path lengths and lower clustering of both functional and structural networks. While in children, higher path lengths and higher clustering were found. What network models can explain these effects? To date several network models have been proposed that can be related to our findings, among those are the small-world model of Watts and Strogatz (Watts and Strogatz, 1998) and the hierarchical modular model (Kaiser et al., 2007).

The Watts and Strogatz model is characterized by one parameter, the randomness, that increases as the network model goes from a regular (or lattice) form to a completely random form. It was shown that path length and cluster coefficient change differentially with the randomness parameter (Watts and Strogatz, 1998). Path length drops exponentially as some edges are rewired randomly in the lattice form, while clustering remains relatively high until the network form is almost completely random (see also Figure 1.6). Therefore, this model is able to differentiate between lattice and random topologies. Where, starting from a small-world model, a lattice form is characterized by higher path length and higher clustering, a more random form is characterized by a decrease in path length and a decrease in clustering. From this point of view, the changes observed in the children with epilepsy can be explained by a shift from a healthy small-world in the control group to a more lattice like form in the patient group. However, it is not straightforward to relate the findings from the adult study to the Watts and Strogatz model. While, an increased path length in the patient group indicated a more lattice like network, a decrease in clustering is characteristic for a more random network topology. It thus seems that this model is not sufficient to explain our findings. This comes as no surprise. It is unlikely that an enormously complex biological system as the human brain is subject to such a simple, one parameter, model. This however does not compromise the usefulness of simple network parameters such as path length and clustering. Rather, a more complex model is needed to explain the observed changes in these parameters.

Network modularity is a topological concept that might be better capable of elucidating some of the observed changes in brain network topology. As shown in Figure 1.6, modularity quantifies the degree to which a network can be divided in groups of nodes that are strongly intra-connected but weakly inter-connected (Newman and Leicht, 2007). A small-world organization is associated with a high modularity: the tightly clustered nodes of the modules give rise to a high cluster coefficient and the between module edges, although sparse, facilitate a low overall path length. However, the degree of clustering and the path length of the within module nodes may vary which would influence the overall values for $L$ and $C$. Furthermore, the between module edges might have an exceptionally large contribution to path length. The deletion (or impairment) of only a few between 
module edges can disconnect two modules which would lead to a large increase in overall path length.

It is from this point of view that we now try to provide a conceptual model for the changes in network parameters found in both groups of epilepsy patients. An explanation for the higher path length and lower cluster coefficient in the adult epilepsy patients could be as follows. The healthy adult brain consists of several modules, where each module is in itself organized as a small-world network. If we now only decrease the amount (or strength) of the between module connections, an increase in path length would result while clustering would be preserved. If now, the within module topology would shift towards a random network, a decrease in clustering would be observed. Here, within module path length would also decrease, but this would be nullified by the loss of between module connectivity, which has a larger impact on the overall path length, see Figure 7.1.

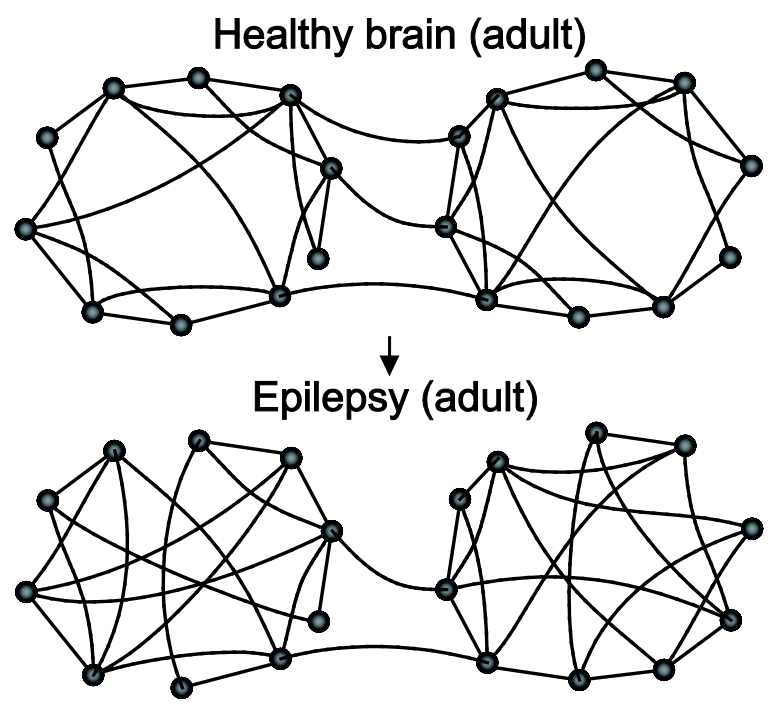

Figure 7.1 Hypothetical model of network changes in adult cryptogenic epilepsy patients. The healthy adult brain (top) is modeled as a small-world modular network. The alteration in the adult patients can be viewed as a randomization of within module connectivity and a reduction in between module connectivity.

In the child group, modularity was directly quantified and was shown to be increased in the patient group. Higher modularity results from a decrease in between module connectivity. This is in line with the increase in path length seen in the patients. As the overall level of connectivity was comparable between the controls and patients, a 
decrease in between module connectivity implies an increase of within module connectivity, which was evident in the increase in clustering, see Figure 7.2.

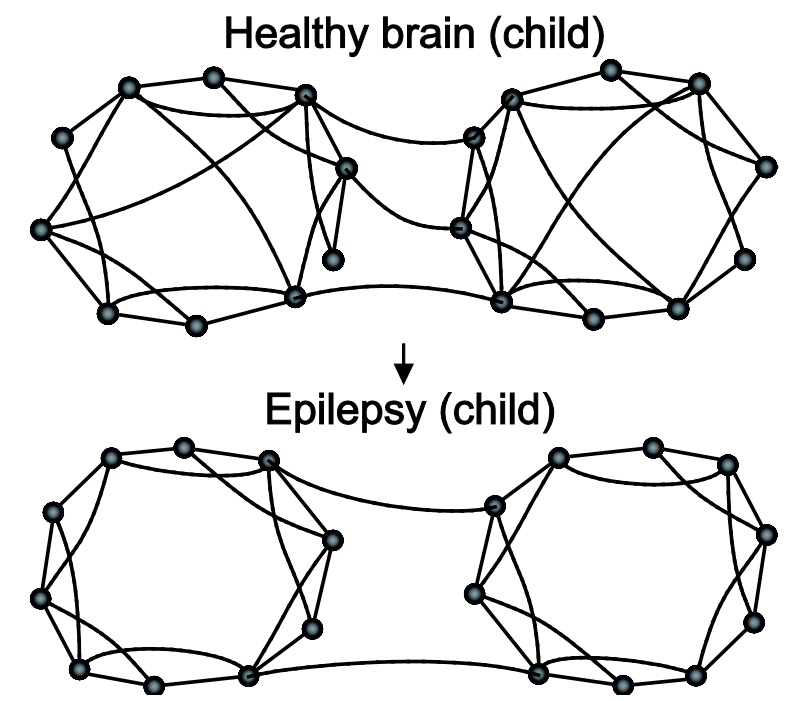

Figure 7.2 Brain networks of children with epilepsy can be thought of as more regular within module networks with reduced between module connectivity.

Thus, two differential effects in terms of small-worldness and modularity can be thought of that might explain the alterations in network parameters found in the adult and child groups. Brain networks of adults with chronic epilepsy suffer from increased modularity and a randomization of within module connectivity. Children with epilepsy have brain networks with a decrease in modularity but a more lattice like within module connectivity (Figure 7.2). Whether brain changes in children should be viewed as developmental or as deterioration is an interesting, yet, unresolved question (Fair et al., 2010; Tosun et al., 2011). However, because of the cross sectional design of our studies, we are currently unable to discriminate between those scenarios. Naturally, the above mentioned theories should not be viewed as definite but rather as a hypothesis that can be tested in detail in future (longitudinal) studies.

\section{The relation between gray and white matter networks in epilepsy}

In the adult patients we found similar network changes for the gray (functional connectivity) and white matter networks (structural connectivity): increased path length and decreased clustering. Changes in both grey and white matter properties have been observed previously in epilepsy (Powell et al., 2007). However, the differences in node definition used in chapters 3 and 4 preclude the direct comparison of network metrics. The gray matter networks were derived from a silent word generation fMRI task and a high resolution network ( 900 nodes), while the white 
matter networks resulted from DWI fiber tractography and a low resolution network ( 90 nodes).

To enable direct comparison of FC (functional connectivity) and SC (structural connectivity) we applied the exact same brain parcellation in the pediatric patient study. Here a high resolution network of 200 nodes was used based on a segmentation of the grey matter and subcortical structures. Network topology of FC was markedly different in the patients, especially the modularity. The SC did not reveal altered topological measures. However, a moderate brain wide increase in white matter connectivity was observed, while the FC revealed an increase in within module connectivity and a decrease in between module connectivity in the patients. One of the advantages of graph analysis is that connection matrices derived from different modalities can be compared directly, as both are modeled by abstract graphs. However, research in this area has only very recently commenced (Hagmann et al., 2010; Honey et al., 2010; Skudlarski et al., 2010; Johansen-Berg, 2011; Zhang et al., 2011b). It has been shown recently that SC is predictive for FC, but that FC in not very predictive for SC (Honey et al., 2010). Thus, there is a prominent role of white matter connectivity for the dynamic correlations across the brain, but the pattern of functional correlations can extend beyond the direct axonal connections (indirect connections). An interesting question is whether changes in SC precede FC changes or vice versa, as this would have implications for the stage of disease at which one of the imaging modalities (probing FC or SC) could detect abnormalities. It is likely that changes in SC would induce changes in FC, as SC is predictive for FC. However, whether changes in $\mathrm{FC}$ also (always) result in altered $\mathrm{SC}$ is unknown.

Currently, there is a lack of biophysical models that describe the relation between FC and SC correlation and pathologically induces alterations (Johansen-Berg, 2011). Therefore, it is difficult to interpret the findings in this thesis from a neurobiological point of view. Future studies applying longitudinal measurements of the FC-SC relation in subjects as well as advances in the understanding of the relation between MR measurements and neurobiology are needed.

\section{The role of graph analysis in neurological and psychiatric disorders}

In this thesis we have proposed that cognitive dysfunction is related to network abnormalities in patients with epilepsy. From a network point of view, higher cognitive functions arise from the orchestrated activity of a large ensemble of cortical regions. Therefore, any abnormalities in either a network node (a cortical region or sub-cortical nuclei) or edge (axonal connections in the white matter) may lead to a dysfunctional network and consequently to cognitive impairment. In what follows, we explore the notion that the relation between network dysfunction and cognitive impairment is also applicable to other neurological and psychiatric disorders and advocate the relevance of several properties of graph analysis. 
Many psychiatric and neurological disorders including epilepsy are characterized by a heterogeneous expression of cognitive problems (Berg, 2011; Natanen et al., 2011). If network abnormalities are at the basis of these cognitive problems, these should be detectable by imaging methods probing network integrity and be related to cognitive and clinical variables of interest. A number of recent studies have applied graph theoretical analysis of brain networks in several neurological and psychiatric disorders. Increased path length has been associated with a decrease in cognitive performance in AD (Stam et al., 2007). A longer duration of illness was associated with increased $L$ and decreased $C$ in schizophrenia (Liu et al., 2008). Also in schizophrenia, IQ scores were related to an increase in $L$ and decrease in $C$ (Zalesky et al., 2011). In major depression a relation between regional network characteristics and duration of illness and cognitive scores was shown (Zhang et al., 2011a). In idiopathic generalized epilepsy, an association between duration of epilepsy and regional network characteristics was also identified (Zhang et al., 2011b). Although differences in pathology, imaging modality and analysis methods hinder the direct comparison of these results, there indeed appears to be a relation between network organization and cognitive and clinical variables in a variety of neurological and psychiatric disorders that can be detected by graph analysis. But what does graph analysis have to offer over more conventional image analysis methods?

Many imaging studies to date have focused on finding the location of tissue abnormalities in clinical populations. Due to the high variability of brain morphology across individuals and the limited signal to noise ratio of the imaging methods (e.g. scans used in $\mathrm{FMRI}$ en DWI) group comparisons are made to obtain sufficient statistical power to detect abnormalities. Implicit to this approach is the assumption that the tissue abnormalities are indeed located at more or less the same anatomical location across individuals (i.e. overlap). Furthermore, it has to be assumed that all individuals in the patient group have to display a similar effect of tissue parameters values (e.g. activation levels or FA values): the mean of the parameter values has to be either sufficiently increased or decreased over the entire patient group to detect significant differences.

First, in the case of patients with cryptogenic localization related epilepsy several arguments can be thought of that disagree with these assumptions. As the label 'cryptogenic' indicates, it is uncertain what the nature of the lesion is, where it is located precisely and whether tissue abnormalities extend beyond the seizure focus. This violates the first assumption: it is not clear why tissue abnormalities should be anatomically co-located at the group level. Indeed, results from the populations studied in this thesis reveal that conventional voxel based comparisons did not reveal significant group differences in functional activation values in adult (Vlooswijk et al., 2010b) and pediatric (Braakman et al., 2011) patients compared to healthy controls. The same was observed for voxelwise comparisons of FA values in pediatric epilepsy patients (unpublished results) where no group differences could be found. From this 
we can carefully conclude that co-localized tissue abnormalities at the group level are not very salient in these patient populations.

Second, cognitive dysfunction in epilepsy has been associated with a change in functional activation patterns (Vlooswijk et al., 2010a), where activation is shifted inter- or intra-hemispherically from the normally activated regions to other cerebral regions. This implies that some functional connections will show a decrease in strength (those engaged with the pathological regions), while others will be increased in strength (those engaged with the atypically activated regions). A similar effect may be seen for measures of microstructure, such as FA or ADC values. This is in line with chapters 5 and 6, where indeed both increases and decreases in connectivity were found, and several publications in other fields were both increases and decreases of tissue parameters have been observed (Powell et al., 2007; Fair et al., 2010; Meng et al., 2010; Zhang et al., 2011a). One could hypothesize that the pattern or reorganization is highly individual and that it is a-priori unknown whether certain tissue parameters will shown an increase or decrease in the individual patient. Therefore, simple statistics that test for differences in mean value of localized abnormalities at the group level might have limited statistical power in neurological disorders with heterogeneous expression of cognitive co-morbidity as observed in epilepsy.

From these points of view, graph theoretical analysis of brain networks might have some conceptual advantages over conventional methods. Whole brain graph measures are derived from an individual brain. Therefore, there is no need to compare voxel-wise parameters at the group level. Different subjects can have very dissimilar brain abnormalities in terms of location and quantitative parameters (higher or lower), which would normally remain undetected by conventional analysis methods. However, these dissimilar abnormalities might display similar graph metrics. As shown in Figure 7.3, two hypothetical subjects with non-overlapping abnormalities can have a similar alteration of graph metrics compared to a normal subject. 
B

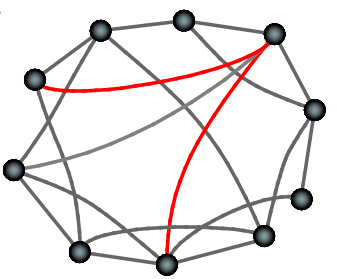

$C=0.18$

$L=1.48$
A

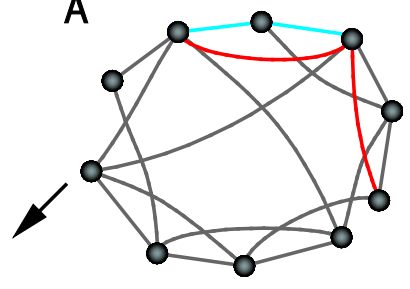

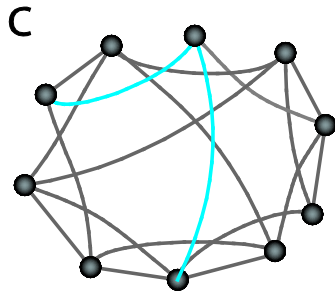

$C=0.23$

$L=1.47$

Figure 7.3 This figure displays three hypothetical brain networks. The one in the middle (A) is assumed to be healthy, while the networks to the left (B) and right (C) are supposed to be pathological networks. In the left network, a rewiring has occurred (red edges) resulting in a decrease of clustering, but similar $L$, compared to the normal network. The network on the right also has a rewiring (blue edges), although a different parts of the network are implicated. Still, a similar reduction in clustering results in (C) as in (B).

\section{Methodological and technical considerations}

\section{Node definition of the brain network}

Modeling the brain as a graph entails the definition of nodes and edges. However, defining nodes is not straightforward. Ideally, these nodes should represent real items of the system. At the macroscopic scale, nodes should correspond to functional cortical areas and sub-cortical nuclei, but the number and exact location of these entities in humans is largely unknown. To date, several approaches of node definitions have been reported (Wang et al., 2010), ranging in size and number from single voxels (van den Heuvel et al., 2008), to small cortical patches (Hagmann et al., 2008; Meunier et al., 2009) or macro-scaled regions from an anatomical atlas (Salvador et al., 2005; Zhang et al., 2011b). Several studies have investigated the effect of different node definitions in detail (Wang et al., 2009; Zalesky et al., 2010; Bassett et al., 2011; Power et al., 2011). In this thesis, we have used several node definitions. In chapter 4, the Automated Anatomical Labeling (AAL) atlas was used with 90 regions. In chapter 3, a refinement of the AAL atlas into $\sim 900$ regions was applied. In chapter 5 the cortical and subcortical atlas from the Freesurfer routines was utilized, containing 82 regions. In chapter 6 , this Freesurfer atlas was refined into $\sim 200$ regions. See Figure 7.4 for an overview of the atlases. 
A

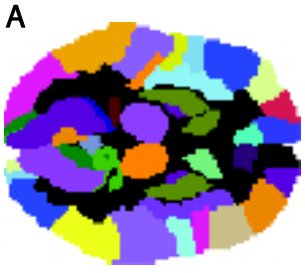

$\mathrm{N}=90$

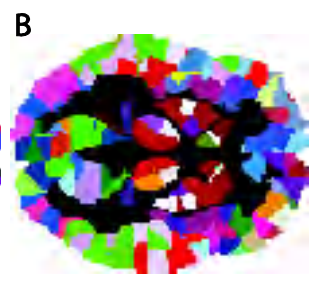

$\mathrm{N}=898$
C

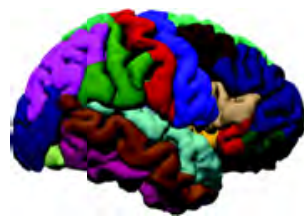

$\mathrm{N}=82$
D

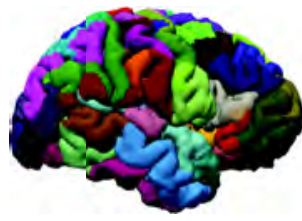

$\mathrm{N}=205$

Figure 7.4 Overview of the different anatomical atlases applied. (A) The AAL atlas with 90 regions. (B) The AAL atlas refined to 898 regions. (C) The default Freesurfer atlas with 82 regions. (D) The refined Freesurfer atlas with 205 regions.

In Zalesky et al. (Wang et al., 2009; Zalesky et al., 2010) it was shown that the number of nodes in the network has an effect on network metrics, but in all cases the measured networks displayed robust small-world characteristics. In (Zhang et al., 2011b) two different network parcellations were applied in a clinical population of epilepsy patients. Results indicated that although exact values of network metrics were different between the two parcellations, the qualitative group differences between the patients and controls were highly similar. The networks metrics from chapter 5 and chapter 6 are also directly comparable as they are derived from the same dataset. Functional network metrics from the Freesurfer atlas and a refined version thereof displayed very similar effects when comparing the different subject groups. From these results we can conclude that although the precise definition of nodes is an important factor that affects the values of network metrics, the interpretation of between group effects appear to be robust to different node definitions.

\section{Binary, weighted, undirected or directed networks}

The edges of a graph can be binary, weighted, undirected or directed. In this thesis the brain has been modeled as an undirected binary or weighted graph. In a binary graph, an edge is either present or not and no further information is stored. In a weighted graph a value is assigned to each edge that represents its connection strength. In chapters 2 and 5 binary graphs were used, whereas in chapters 3, 4, 5 and 6 the weighted variant was applied. Although there are several conceptual differences between binary and weighted graphs, such as the amount of information that is retained in the graph, it is unclear which of the two methods is most sensitive as a biomarker. In chapter 5, the two were directly compared and although some minor differences were visible, the overall interpretation of the results was similar. This would indicate that both methods also have similar sensitivity, although further research is needed to be able to extend this finding to other pathologies.

A related issue is the choice of edge weight in FC and SC. In SC this choice is less straightforward than in FC, where the correlation value is most often used. Other measures of functional coupling, such as partial correlation values (correlation where 
the influence of several other variables are filtered out, e.g. the indirect correlation of two regions that are caused by a third region) or more advanced methods may also be used (Smith et al., 2011), although these remain largely unexplored in the framework of graph analysis. Furthermore, some concerns about the sensitivity and specificity of several methods have been raised recently (Smith et al., 2011). For SC, the choice of weighting is not straightforward. Several options can be explored, such as the number of streamlines, the (relative) volume of the tract or the mean FA or ADC value of the voxel within the tract. Some authors have even opted to combine several of these measures and also to include edge length in the weighting scheme (Hagmann et al., 2008; Zhang et al., 2011b). Currently, there is no consensus on which weighting scheme best captures the efficacy of a white matter tract. This issue will likely remain unresolved, as no 'gold standard' for fiber tractography is available for the human brain. Studies combining chemical tracers and electro-stimulation or optogenetic methods (Deisseroth, 2011) in animals could potentially shed light on this issue.

Directed networks are also of high interest in neuroscience. Ideally, the direction of a cortico-cortical or cortico-subcortical connection would provide information on the flow of information between neurons. Currently, deriving directed networks from DWI data is not possible. Fiber tractography is unable to differentiate either afferent or efferent structural connections. Several methods to obtain the directionality of a connection from functional MRI data exist and are still being developed. Such methods include Structural Equation Modeling (SEM) (Bullmore et al., 2000), Granger Causality Mapping (GCM) (Roebroeck et al., 2005) and Dynamic Causal Modeling (DCM) (Friston et al., 2003). In their current form SEM and DCM are not applicable to large networks, as methodological and computational limitations hinder the inference of model parameters for a large number of regions. However, it would be interesting to investigate the added value of whole brain GCM analysis with graph theoretical parameters for directed networks (Liao et al., 2011) (e.g. the causal relationships between sub networks could be characterized).

\section{Functional connectivity: Resting state versus task fMRI}

In this thesis, the most straightforward implementation of functional connectivity was used: the linear correlation between the BOLD signal time series of two regions. In chapter 3, the BOLD signal time series were extracted from an fMRI experiment in which subjects performed a word generation task in the scanner. In chapters 5 and 6 , a resting state $\mathrm{fMRI}$ experiment was used. In both cases the concept of functional connectivity is the same, what regions in the brain co-activate? However, it is not clear what the correspondence between the architecture of functional networks during rest or task is and how task performance influences group differences in network metrics. Several recent studies have investigated this in more detail using Independent Component Analysis (ICA) (Calhoun et al., 2008; Smith et al., 2009). In both studies it was shown that the networks identified with ICA were present in both task and rest data. This indicates that the brain is composed of several sub networks 
that are functionally linked and, over the course of several minutes, synchronize their activity whether the owner of the brain is performing a task or not. For instance, in (Calhoun et al., 2008), components representing the motor and visual systems could be successfully identified both during an auditory oddball task and resting state. An ICA analysis of our data showed similar results, the task network of the word generation was also evident in the resting state data, as shown in Figure 7.5.

(A) Resting State ICA

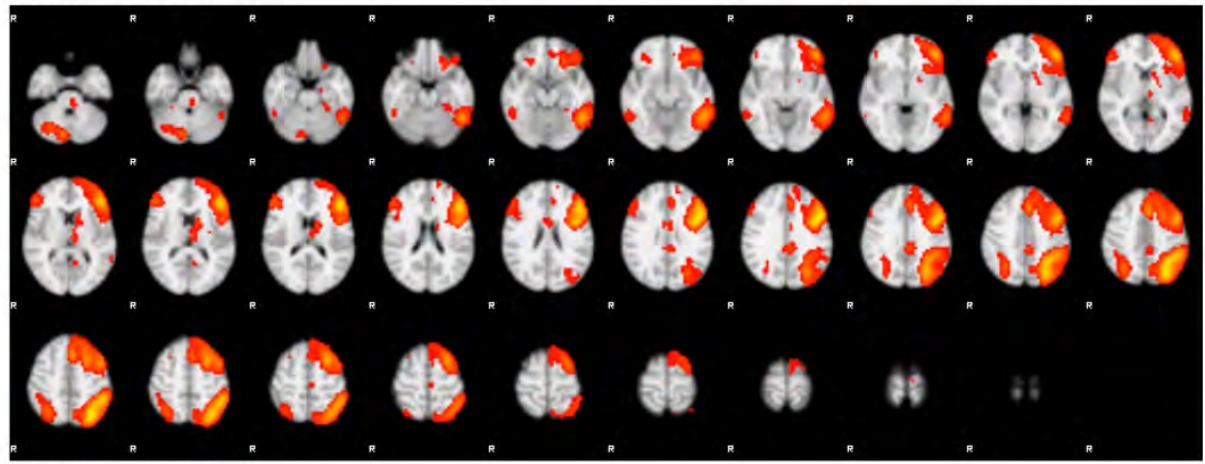

(B) Word generation ICA

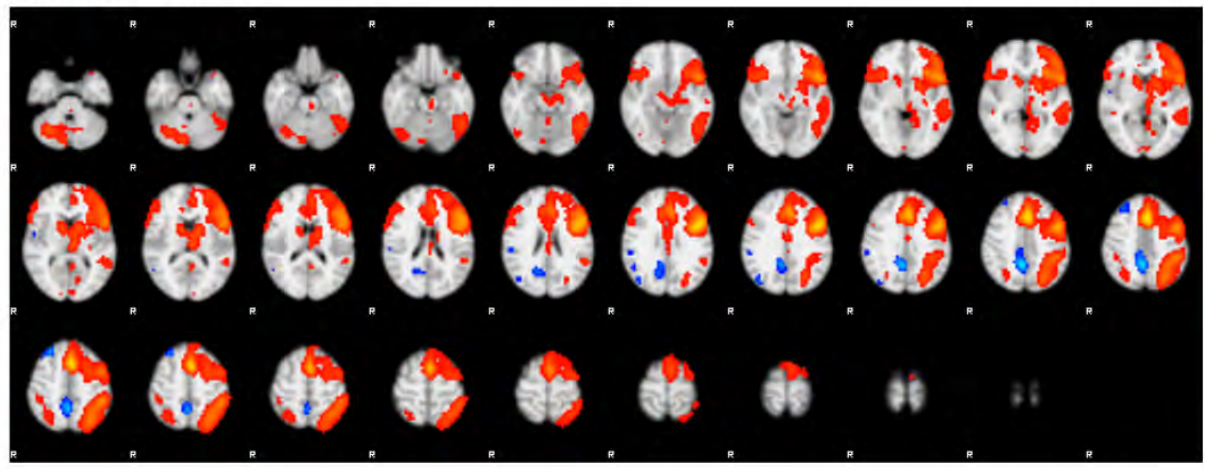

Figure 7.5 Functional networks from resting state and task data. The top panel shows a resting state network involving left lateralized frontal and parietal regions. The bottom panel displays a task network found to be activated by the word generation task. Note the striking similarity between the two networks.

To date, no studies have directly compared functional graph metrics between task and resting state. However, the above mentioned studies and the results from chapter 3 (task) and chapter 5 (resting state) indicate that, on a whole brain scale, highly similar results should be expected. Although the results from chapters 3 and 5 cannot be directly compared due to differences in study population and node definition, it is apparent that functional whole brain networks during task or rest display efficient small world properties. It is likely that the performance of a task amplifies the 
correlation values of the regions involved in the task. However, this apparently does not mean that dynamic fluctuations in other brain regions are attenuated such that no functional connectivity can be detected. Therefore, a relevant question is whether task $\mathrm{fMRI}$ is needed at all when investigating functional networks in neurological disorders. As shown above, many of the networks determined with task fMRI are also present in resting state $\mathrm{fMRI}$. However, an interesting hypothesis is that some disease related effects only become visible during cognitive effort (Meda et al., 2009). Therefore, it would be appealing to investigate functional connectivity at different cognitive efforts. Also, it would be of interest to investigate whether individual differences in network topology during rest and task would be characteristic for neurological disorders. A recently proposed theory (Menon, 2011) suggests that abnormalities in the interplay between several core functional networks (default mode network, central executive network and the saliency network) form the basis for cognitive dysfunction in a large variety of neurological and psychiatric disorders. Such abnormalities might be detected by comparing metrics of functional network organization during rest and task. Furthermore, resting state $\mathrm{fMRI}$ is also applicable in clinical populations where task compliance is a problem (e.g. very young patients). On the other hand, task fMRI might be better capable of localizing specific areas at the individual level. These could then be used as seed ROIs for connectivity analyses.

\section{DWI and crossing fibers}

Fiber tractography enables the delineation of the white matter into large fiber bundles. Over the past decade a large number of methods have been developed to characterize the orientation of fibers from DWI data. In this thesis, two different types of fiber models have been used: the diffusion tensor model and the spherical deconvolution model. In diffusion tensor tractography, the principal diffusion direction of the tensor is used as fiber direction. Thus, only one fiber direction can be modeled per voxel, even though crossing fibers are prevalent in the human brain. In chapters 2 and 4, a probabilistic extension of this model was used to perform fiber tractography (Parker et al., 2003). For this the amount of anisotropy in the tensor was used to infer a probability function for the principal diffusion direction. In some cases this can reduce the shortcomings of the single tensor model in crossing fibers, e.g. in a voxel with two crossing fiber bundles the probabilistic model of the principal diffusion direction will allow a tract to navigate through this voxel. However, an explicit model of multiple fiber direction is more appropriate. Therefore, in chapter 6, the constrained spherical deconvolution model was used (Tournier et al., 2007). This model allows the estimation of the fiber orientation distribution at high angular resolution and is capable of tracking through white matter regions with crossing fibers.

DWI tractography is still a very active research field and many innovations are still being made, not only for fiber models but also at the level of image acquisition and post-processing (Jones, 2010). Until a golden standard for human white matter neuro- 
anatomy is provided, it will remain difficult to precisely evaluate the pros and cons of the different fiber models and tractography algorithms. However, a more relevant question here is whether the choice for a certain method could introduce a bias when investigating different clinical populations. No reports on this subject have been published and it is unlikely that group differences will depend on the choice of method. Therefore, even though the different fiber models have several conceptual advantages and disadvantages, all can be used in clinical studies. The sensitivity of different methods in different neurological disorders is as of yet largely unknown, but would be of importance for future study designs.

\section{Clinical implications and future perspectives}

Based on MR network imaging, one would like to provide a prognosis based on the neurological substrate to determine the individual patient's vulnerability for cognitive decline. This would entail interpretation of the individual patient's MR data. Currently, the available MR connectivity measurements do not seem ready for this and the cognitive consequences of epilepsy can only be detected with neuropsychological assessment in patients who already experience problems in daily life. However, clinicians are unable to predict which patient will be at risk to develop cognitive problems based only on clinical variables. Therefore, research aimed at finding early MR biomarkers for development of cognitive problems should continue.

The results presented in this thesis demonstrate that a mechanism underlying or at least associated with cognitive dysfunction in cryptogenic localization-related epilepsy appears to be a disruption of large-scale brain networks. When changes in MR network parameters would precede changes in neuropsychological parameters, this would potentially open a window towards early detection of neuronal changes that increase the risk for the development of cognitive impairment. The early detection of patients at risk may be valuable, especially in chronic epilepsy, as earlier therapeutic interventions hopefully lead to a better neurocognitive outcome at long term. However, several advances are needed before such scenarios might become reality. MRI protocols need to be optimized and preferably standardized, such that results from different study and research centers can be compared and aggregated. This would also require a level of standardization of post-processing protocols. In functional mapping, standardized tools are already available (e.g. SPM and FSL), although no such tools are currently available for resting state fMRI connectivity or DWI tractography, but will likely become available in the near future.

Although a comparison between epilepsy patients with epilepsy from different age categories was made in this discussion, ideally longitudinal studies should be performed to monitor the relation between neuronal organization, cognitive dysfunction and clinical factors. Theoretically, patients should be followed even before the onset of epilepsy, although this is obviously practically infeasible. Therefore, a realistic option would be to follow patients from the onset of epilepsy 
over large time periods. Such studies might be able to provide unique insight on the causal relations between seizures, brain abnormalities and cognitive deficits. Also, as the influence of clinical factors on brain organization and cognition is likely to be multi-factorial, large cohorts are needed to have sufficient statistical power to disentangle this interaction. 


\section{REFERENCES}

Bassett DS, Brown JA, Deshpande V, Carlson JM, Grafton ST (2011) Conserved and variable architecture of human white matter connectivity. Neuroimage 54:1262-1279.

Berg AT (2011) Epilepsy, cognition, and behavior: The clinical picture. Epilepsia 52 Suppl 1:7-12.

Berl MM, Balsamo LM, Xu B, Moore EN, Weinstein SL, Conry JA, Pearl PL, Sachs BC, Grandin CB, Frattali C, Ritter FJ, Sato S, Theodore WH, Gaillard WD (2005) Seizure focus affects regional language networks assessed by fMRI. Neurology 65:1604-1611.

Bonelli SB, Powell RH, Yogarajah M, Samson RS, Symms MR, Thompson PJ, Koepp MJ, Duncan JS (2010) Imaging memory in temporal lobe epilepsy: predicting the effects of temporal lobe resection. Brain 133:1186-1199.

Braakman H, Vaessen M, Jansen J, Hofman P, Aldenkamp A, Backes W (2011) Functional connectivity of the language network in children with Frontal Lobe Epilepsy. in preparation.

Bullmore E, Horwitz B, Honey G, Brammer M, Williams S, Sharma T (2000) How good is good enough in path analysis of $\mathrm{FMRI}$ data? Neuroimage 11:289-301.

Calhoun VD, Kiehl KA, Pearlson GD (2008) Modulation of temporally coherent brain networks estimated using ICA at rest and during cognitive tasks. Hum Brain Mapp 29:828-838.

Cheung MC, Chan AS, Chan YL, Lam JM, Lam W (2006) Effects of illness duration on memory processing of patients with temporal lobe epilepsy. Epilepsia 47:1320-1328.

Deisseroth K (2011) Optogenetics. Nat Methods 8:26-29.

Fair DA, Posner J, Nagel BJ, Bathula D, Dias TG, Mills KL, Blythe MS, Giwa A, Schmitt CF, Nigg JT (2010) Atypical default network connectivity in youth with attention-deficit/hyperactivity disorder. Biological psychiatry 68:1084-1091.

Focke NK, Yogarajah M, Bonelli SB, Bartlett PA, Symms MR, Duncan JS (2008) Voxel-based diffusion tensor imaging in patients with mesial temporal lobe epilepsy and hippocampal sclerosis. Neuroimage 40:728-737.

Friston KJ, Harrison L, Penny W (2003) Dynamic causal modelling. Neuroimage 19:1273-1302.

Hagmann P, Cammoun L, Gigandet X, Meuli R, Honey CJ, Wedeen VJ, Sporns O (2008) Mapping the structural core of human cerebral cortex. PLoS Biol 6:e159.

Hagmann P, Sporns O, Madan N, Cammoun L, Pienaar R, Wedeen VJ, Meuli R, Thiran JP, Grant PE (2010) White matter maturation reshapes structural connectivity in the late developing human brain. Proc Natl Acad Sci U S A 107:19067-19072.

Honey CJ, Thivierge JP, Sporns O (2010) Can structure predict function in the human brain? Neurolmage 52:766-776.

Johansen-Berg H (2011) The future of functionally-related structural change assessment. Neurolmage.

Jones DK (2010) Diffusion MRI: Theory, Methods, and Applications: Oxford University Press.

Kaiser M, Goerner M, Hilgetag CC (2007) Criticality of spreading dynamics in hierarchical cluster networks without inhibition. New Journal of Physics 9:110.

Liao W, Ding J, Marinazzo D, Xu Q, Wang Z, Yuan C, Zhang Z, Lu G, Chen H (2011) Small-world directed networks in the human brain: multivariate Granger causality analysis of resting-state fMRI. Neuroimage 54:2683-2694.

Liu Y, Liang M, Zhou Y, He Y, Hao Y, Song M, Yu C, Liu H, Liu Z, Jiang T (2008) Disrupted small-world networks in schizophrenia. Brain 131:945-961. 
Meda SA, Stevens MC, Folley BS, Calhoun VD, Pearlson GD (2009) Evidence for anomalous network connectivity during working memory encoding in schizophrenia: an ICA based analysis. PLoS One 4:e7911.

Meng L, Xiang J, Kotecha R, Rose D, Zhao H, Zhao D, Yang J, Degrauw T (2010) White matter abnormalities in children and adolescents with temporal lobe epilepsy. Magnetic resonance imaging 28:12901298.

Menon V (2011) Large-scale brain networks and psychopathology: a unifying triple network model. Trends in cognitive sciences 15:483-506.

Meunier D, Lambiotte R, Fornito A, Ersche KD, Bullmore ET (2009) Hierarchical modularity in human brain functional networks. Front Neuroinform 3:37.

Naatanen R, Kujala T, Kreegipuu K, Carlson S, Escera C, Baldeweg T, Ponton C (2011) The mismatch negativity: an index of cognitive decline in neuropsychiatric and neurological diseases and in ageing. Brain.

Newman ME, Leicht EA (2007) Mixture models and exploratory analysis in networks. Proc Natl Acad Sci U S A 104:9564-9569.

Parker GJ, Haroon HA, Wheeler-Kingshott CA (2003) A framework for a streamline-based probabilistic index of connectivity (PICo) using a structural interpretation of MRI diffusion measurements. Journal of magnetic resonance imaging : JMRI 18:242-254.

Powell HW, Parker GJ, Alexander DC, Symms MR, Boulby PA, Wheeler-Kingshott CA, Barker GJ, Koepp MJ, Duncan JS (2007) Abnormalities of language networks in temporal lobe epilepsy. Neuroimage 36:209-221.

Power Jonathan D, Cohen Alexander L, Nelson Steven M, Wig Gagan S, Barnes Kelly A, Church Jessica A, Vogel Alecia C, Laumann Timothy O, Miezin Fran M, Schlaggar Bradley L, Petersen Steven E (2011) Functional Network Organization of the Human Brain. Neuron 72:665-678.

Riley JD, Franklin DL, Choi V, Kim RC, Binder DK, Cramer SC, Lin JJ (2010) Altered white matter integrity in temporal lobe epilepsy: association with cognitive and clinical profiles. Epilepsia 51:536-545.

Roebroeck A, Formisano E, Goebel R (2005) Mapping directed influence over the brain using Granger causality and fMRI. Neuroimage 25:230-242.

Salvador R, Suckling J, Coleman MR, Pickard JD, Menon D, Bullmore E (2005) Neurophysiological architecture of functional magnetic resonance images of human brain. Cereb Cortex 15:13321342 .

Skudlarski P, Jagannathan K, Anderson K, Stevens MC, Calhoun VD, Skudlarska BA, Pearlson G (2010) Brain connectivity is not only lower but different in schizophrenia: a combined anatomical and functional approach. Biological psychiatry 68:61-69.

Smith SM, Miller KL, Salimi-Khorshidi G, Webster M, Beckmann CF, Nichols TE, Ramsey JD, Woolrich MW (2011) Network modelling methods for FMRI. Neuroimage 54:875-891.

Smith SM, Fox PT, Miller KL, Glahn DC, Fox PM, Mackay CE, Filippini N, Watkins KE, Toro R, Laird AR, Beckmann CF (2009) Correspondence of the brain's functional architecture during activation and rest. Proc Natl Acad Sci U S A 106:13040-13045.

Stam CJ, Jones BF, Nolte G, Breakspear M, Scheltens P (2007) Small-world networks and functional connectivity in Alzheimer's disease. Cereb Cortex 17:92-99.

Tosun D, Dabbs K, Caplan R, Siddarth P, Toga A, Seidenberg M, Hermann B (2011) Deformation-based morphometry of prospective neurodevelopmental changes in new onset paediatric epilepsy. Brain 134:1003-1014. 
Tournier JD, Calamante F, Connelly A (2007) Robust determination of the fibre orientation distribution in diffusion MRI: non-negativity constrained super-resolved spherical deconvolution. Neurolmage 35:1459-1472.

van den Heuvel MP, Stam CJ, Boersma M, Hulshoff Pol HE (2008) Small-world and scale-free organization of voxel-based resting-state functional connectivity in the human brain. Neuroimage 43:528-539.

Vlooswijk MC, Jansen JF, de Krom MC, Majoie HM, Hofman PA, Backes WH, Aldenkamp AP (2010a) Functional MRI in chronic epilepsy: associations with cognitive impairment. Lancet neurology 9:1018-1027.

Vlooswijk MC, Jansen JF, Majoie HJ, Hofman PA, de Krom MC, Aldenkamp AP, Backes WH (2010b) Functional connectivity and language impairment in cryptogenic localization-related epilepsy. Neurology 75:395-402.

Wang J, Zuo X, He Y (2010) Graph-based network analysis of resting-state functional MRI. Front Syst Neurosci 4:16.

Wang J, Wang L, Zang Y, Yang H, Tang H, Gong Q, Chen Z, Zhu C, He Y (2009) Parcellation-dependent smallworld brain functional networks: a resting-state fMRI study. Hum Brain Mapp 30:1511-1523.

Watts DJ, Strogatz SH (1998) Collective dynamics of 'small-world'networks. Nature 393:440-442.

Weber B, Wellmer J, Schur S, Dinkelacker V, Ruhlmann J, Mormann F, Axmacher N, Elger CE, Fernandez G (2006) Presurgical language fMRI in patients with drug-resistant epilepsy: effects of task performance. Epilepsia 47:880-886.

Yogarajah M, Powell HW, Parker GJ, Alexander DC, Thompson PJ, Symms MR, Boulby P, Wheeler-Kingshott CA, Barker GJ, Koepp MJ, Duncan JS (2008) Tractography of the parahippocampal gyrus and material specific memory impairment in unilateral temporal lobe epilepsy. Neuroimage 40:17551764.

Zalesky A, Fornito A, Harding IH, Cocchi L, Yucel M, Pantelis C, Bullmore ET (2010) Whole-brain anatomical networks: does the choice of nodes matter? Neuroimage 50:970-983.

Zalesky A, Fornito A, Seal ML, Cocchi L, Westin CF, Bullmore ET, Egan GF, Pantelis C (2011) Disrupted axonal fiber connectivity in schizophrenia. Biol Psychiatry 69:80-89.

Zhang J, Wang J, Wu Q, Kuang W, Huang X, He Y, Gong Q (2011a) Disrupted brain connectivity networks in drug-naive, first-episode major depressive disorder. Biol Psychiatry 70:334-342.

Zhang Z, Liao W, Chen H, Mantini D, Ding JR, Xu Q, Wang Z, Yuan C, Chen G, Jiao Q, Lu G (2011b) Altered functional-structural coupling of large-scale brain networks in idiopathic generalized epilepsy. Brain : a journal of neurology 134:2912-2928. 
$162 \mid$ Chapter 7 
Summary 


\section{SUMMARY}

Many patients with epilepsy develop cognitive problems in the course of their disease, such as memory problems, slowness of thought or problems finding words. The etiology of these cognitive problems has not been resolved. It is thought that cognitive functioning is the result of interactions between brain areas in large scale networks. Therefore, it is interesting to investigate whether the cause of cognitive problems results from a disruption of brain networks. With the aid of relatively new MRI techniques, in particular functional Magnetic Resonance Imaging (fMRI) and diffusion weighted MRI (DWI), it is possible to measure abnormalities of brain networks which might be related to cognitive impairments. The research in this thesis has focused on detecting network abnormalities in patients with epilepsy compared to healthy controls. The results demonstrated that patients with epilepsy - especially those with cognitive problems - have a disrupted organization of functional and structural networks. These findings are an important step in understanding the neuronal correlate of cognitive deficits in neurological diseases such as epilepsy.

Epilepsy is one of the most prevalent neurological disorders worldwide. It affects 1-2\% percent of the population at some point in their lives. In the Netherlands alone, approximately 110,000 persons have some form of epilepsy at any given moment. The unpredictability and sudden occurrence of a seizure frequently creates a great social burden to the patient and those who surround him. Although the direct consequences of epileptic seizures are serious, many patients rank their cognitive impairments highest on their list of complaints. Much research has focused on clinical factors that might contribute to the development of cognitive impairments, for instance, the role of seizure frequency, age at onset of the seizures and anti-epileptic drug use. However, results have been mixed and thus far no conclusive relationship between cognitive problems and these clinical factors has been demonstrated. At present, it is difficult for the clinician to give a prognosis: patients and relatives cannot be informed about whether cognition will remain intact and whether a certain anti-epileptic drug (AED) will successfully control seizures. Therefore, diagnostic tools to identify patients at risk of cognitive impairment and treatment failure are needed.

Owing to the recent progress made in $M R$ imaging and particularly computational methods, it is now possible to measure how macroscopic brain regions functionally interact and how they are connected by white matter fiber bundles. Functional MRI allows indirect measurement of neuronal activity in the brain's grey matter. Functional connectivity can then be derived by calculating the synchronicity between the activity of different brain areas. Diffusion weighted MRI is able to provide information on the micro structural organization of the white matter (the nerve fibers). By applying fiber tractography it is possible to reconstruct and visualize structural connections between brain areas. This opens up new opportunities for 
researchers to investigate the properties of the human brain network, how they relate to cognitive functioning, and how connectivity is affected by cerebral diseases such as epilepsy. Intuitively, a network is a collection of entities that are somehow connected and thus the term network can be applied to a large number of systems like the brain, the internet, or groups of socially interacting humans. A more formal definition of a network exists from the field of applied mathematics: here a network is often called a graph which is composed of its individual elements, the nodes, and the link between nodes, the edges. At the macroscopic scale the nodes of the brain graph relate to brain regions and the edges represent the functional or structural connectivity between regions. With methods from graph theory, it is possible to derive simple characteristics of enormously complex networks that provide information on the topological properties of the network, thus facilitating research where measured networks need to be compared. A great variety of graph metrics exist. This thesis focuses on metrics that characterize integration and segregation between brain regions (path length and clustering respectively) and sub-networks formed by brain regions (modularity analysis).

The aim of this thesis was to develop and explore the use of graph analysis methods in finding the neuronal correlate of cognitive impairment in epilepsy. Research to date has mainly focused on the relation between cognitive parameters and localized functional and structural tissue abnormalities. Although this approach is viable, in this thesis we advocate a different paradigm: the brain is to be viewed as a highly interconnected system and analysis methods should be tailored towards relating cognitive deficits to characteristics of large-scale networks.

Not much was known about the peculiarities of deriving graph metrics from structural large-scale brain networks. Therefore, in chapter 2, the effect of several imaging parameters on the reproducibility of graph metrics was investigated. The results indicated that the graph metrics were well reproducible and thus might be used to detect abnormalities in brain networks. The reproducibility of graph metrics was better than that of traditional tract measures such as the number of tracts of a connection.

In this thesis, two different groups of patients are investigated and compared to healthy controls. Chapter 3 and 4 are based on the population from the CODICE (COgnitive Deterioration In Cryptogenic Epilepsy) study. In this study adult patients with localization related but cryptogenic epilepsy (i.e. no visible lesions on structural MRI) are included. Although all patients have a seizure onset in the frontal or temporal lobe, their epilepsy is of unknown cause. Chapters 5 and 6 are based on another cohort: the IMAGINE (IMAGing IN Epilepsy) study. Here, children with localization related epilepsy, also without visible lesions on macrostructural MRI, are included. All patients have a seizure focus in the frontal lobe. Graph metrics derived 
from both functional and structural brain networks were compared between patients and healthy controls and related to cognitive performance.

In chapter 3 it was shown that reduced efficiency of cerebral functional networks was evident in adult patients with chronic cryptogenic epilepsy and that the cerebral efficiency was related to measures of global cognitive performance. Especially the patients with severe cognitive problems displayed a less efficient network (increased path length and reduced clustering). Chapter 4 dealt with the same study population, but here structural networks were investigated. Differences in graph metrics between patients with cognitive impairment and healthy controls were identified as well as a strong relation between cognitive scores and graph metrics. Again, the largest deviations in graph metrics were seen in the patients with the most severe cognitive problems. A cohort of children with frontal lobe epilepsy was investigated in chapter 5. Functional brain networks showed connectional abnormalities that were mainly expressed as a reduction in coupling between functional sub-networks (modules) and this reduction was correlated with poorer cognitive function. The reduction in between-module connectivity was most evident in the most cognitively impaired patients. Moreover, the frontal lobe displayed a deviant modular organization in patients. A specific module was identified that occupied several brain lobes in healthy controls but was severely reduced in extent in patients, especially those with cognitive impairment. In chapter 6 we studied whether the functional abnormalities found in children with FLE could also be related to structural networks. A reduction in between-module connectivity with increased modularity, clustering and path length was observed in the patients. However, an overall increase in white matter connectivity was observed, although whole structural graph metrics did not show any differences.

The results presented in this thesis demonstrate that disruption of large-scale brain networks is one of the mechanisms underlying, or at least associated with, cognitive dysfunction in cryptogenic localization-related epilepsy. When changes in MR network measures would precede changes in neuropsychological outcomes, this would potentially open a window towards early detection of neuronal changes that increase the risk for the development of cognitive impairment. The early detection of patients at risk may be valuable, especially in chronic epilepsy, as earlier therapeutic interventions hopefully lead to a better long term neurocognitive outcome. This is particularly important for children with epilepsy, as children and parents remain uncertain about treatment success and the maximum achievable educational level. 
Samenvatting 
170 


\section{SAMENVATTING}

In het verloop van hun ziekte ontwikkelen patiënten met epilepsie vaak cognitieve stoornissen zoals geheugen problemen, traagheid van denken en problemen met het vinden van woorden. De oorzaak van deze cognitieve stoornissen is nog onbekend. $\mathrm{Er}$ wordt gedacht dat cognitieve functies het resultaat zijn van synchrone activiteit tussen gebieden in de hersenen die onderdeel zijn van netwerken. Het is daarom interessant om de oorzaak van cognitieve stoornissen te zoeken in de verstoring van hersennetwerken. Met behulp van relatief nieuwe Magnetic Resonance Imaging (MRI) technieken, in het bijzonder functionele MRI (fMRI) en diffusie gewogen MRI (DWI), is het mogelijk om eigenschappen van netwerken in de hersenen te meten en te onderzoeken of mogelijke afwijkingen in deze netwerkeigenschappen gerelateerd zijn aan de cognitieve stoornissen. Deze thesis richt zich op het detecteren van afwijkingen in hersennetwerken bij patiënten met epilepsie in vergelijking tot gezonde personen. De resultaten demonstreren dat patiënten met epilepsie - vooral diegenen met cognitieve stoornissen - een afwijkende functionele en structurele hersennetwerk organisatie hebben. Deze bevindingen geven inzicht in het neuronale correlaat van cognitieve stoornissen bij neurologische ziekten zoals epilepsie.

Epilepsie is een van de meest voorkomende neurologische ziekten wereldwijd en 1 tot $2 \%$ van de bevolking heeft er op een gegeven moment last van. In Nederland hebben ongeveer 110,000 mensen epilepsie. De onvoorspelbaarheid en plotselinge opkomst van een epileptische aanval zorgt ervoor dat patiënten en hun naasten het sociaal moeilijker hebben. De aanvallen zijn het meest bekende aspect van de ziekte, maar veel patiënten vinden hun cognitieve stoornissen het meest belastend. $\mathrm{Er}$ is al veel onderzoek gedaan naar de relatie tussen klinische factoren en het ontstaan van cognitieve stoornissen, bijvoorbeeld de aanvalsfrequentie, de leeftijd waarop de epilepsie begon en het gebruik van medicatie. Deze onderzoeken laten echter geen eenduidige resultaten zien. Mede hierdoor is het lastig voor de behandelaar om een duidelijke prognose te geven. Daarom is er een behoefte aan additionele diagnostische middelen om patiënten, die cognitieve achteruitgang zullen gaan vertonen, te kunnen identificeren.

Dankzij recente ontwikkelingen in $\mathrm{MRI}$ en beeldanalyse methoden is het nu mogelijk om in kaart te brengen hoe het brein op een grove (macroscopische) schaal functioneel en structureel verbonden is. Met functionele MRI kan indirect de neuronale activiteit in de grijze stof worden gemeten. De functionele connectiviteit kan dan berekend worden aan de hand van de mate van synchronisatie tussen verschillende gebieden. Met diffusie-gewogen MRI kan de microstructurele oriëntatie van de witte stof (de zenuwbanen) bepaald worden, en door fiber tractography toe te passen kan worden berekend en gevisualiseerd hoe gebieden structureel verbonden zijn. Dit geeft onderzoekers nieuwe mogelijkheden om de eigenschappen van 
hersennetwerken te meten, uit te zoeken hoe deze eigenschappen samenhangen met het cognitief functioneren en te bepalen of hersennetwerken aangedaan zijn in neurologische ziekten zoals epilepsie. Een netwerk is een begrip waarmee meestal een verzameling objecten (deelsystemen) wordt aangeduid die op een of andere manier verbonden zijn. De term netwerk slaat dus op een grote verzameling systemen zoals het brein, het internet of de sociale interactie tussen een groep mensen. Vanuit de toegepaste wiskunde bestaat er echter een meer formele definitie van een netwerk, deze wordt dan een graaf genoemd. Een graaf bestaat uit de elementen van het systeem (de nodes, knooppunten) en de verbindingen tussen de nodes (de edges). Macroscopisch kunnen we de nodes toekennen aan brein regio's en de edges toekennen aan de functionele of structurele verbindingen tussen de verschillende regio's in het brein. Met behulp van methodes uit de graaf theorie kunnen we een complex netwerk samenvatten in een klein aantal maten (graaf maten) die ons een beeld geven over de opbouw (topologie) van het netwerk. Op deze manier wordt het uitvoeren van wetenschappelijk onderzoek naar complexe netwerken gefaciliteerd. Er bestaan een groot aantal graaf theoretische maten. In deze thesis wordt vooral gebruik gemaakt van maten die informatie geven over de integratie en segregatie tussen brein regio's (respectievelijk pad lengte en clustering) en over hoe het brein opgedeeld is in subnetwerken (modulariteit).

Het primaire doel van het onderzoek in deze thesis was het ontwikkelen en toepassen van graaf theoretische analyse methodes om het neuronale correlaat van cognitieve stoornissen in epilepsie te vinden. Tot nu toe hebben veel studies zich gericht op het vinden van een specifieke locatie in het brein waar het weefsel aangedaan is. Dit is zeker een praktische aanpak, maar in deze thesis hebben we gepleit voor een ander paradigma waarbij het brein wordt gezien als een sterk verbonden systeem en geanalyseerd zou moeten worden met methodes die netwerk- en cognitieve eigenschappen direct kunnen relateren.

Het onderzoek in deze thesis vergelijkt de resultaten tussen twee verschillende groepen: patiënten met epilepsie en gezonde personen. In de hoofdstukken 3 en 4 worden mensen uit de CODICE (COgnitive Deterioration In Cryptogenic Epilepsy) studie onderzocht. Deze groep bestaat uit patiënten met cryptogene lokalisatie gebonden epilepsie (d.w.z. er zijn geen grote laesies te zien op standaard MRI) en een groep gezonde volwassenen van vergelijkbare leeftijd. De patiënten hebben een epileptisch focus in de frontaal kwab of temporaalkwab, maar de precieze oorzaak van de epilepsie is onbekend. De hoofdstukken 5 en 6 zijn gebaseerd op de IMAGINE (IMAGing IN Epilepsy) studie waarin jonge kinderen met lokalisatie gebonden epilepsie zijn onderzocht. Deze patiëntjes hebben geen zichtbare afwijkingen op structurele MRI en het epileptisch focus bevindt zich in de frontaal kwab. Bij deze groepen zijn graaf maten van de functionele en structurele hersennetwerken 
berekend en vergeleken met de gezonde controles en gerelateerd aan cognitief presteren.

Omdat er nog weinig bekend was over het berekenen van graaf maten van structurele netwerken, zijn het effect van MRI scan parameters op graaf maten en de reproduceerbaarheid van de graaf maten onderzocht in hoofdstuk 2 . Hier bleek dat deze parameters inderdaad goed reproduceerbaar waren en dus mogelijk gebruikt konden worden om afwijkingen te detecteren, beter dan traditionele maten zoals het aantal tracts. De resultaten beschreven in hoofdstuk 3 lieten zien dat de functionele netwerken minder efficiënt waren opgebouwd bij de volwassen epilepsie patiënten en dat de graaf maten gecorreleerd waren aan cognitief functioneren. Vooral de patiënten met een meer uitgesproken intellectuele achteruitgang lieten een minder efficiënte opbouw van het netwerk zien (langere padlengte en verminderde clustering). In hoofdstuk 4 zijn dezelfde patiënten en controles onderzocht, maar hier zijn juist de eigenschappen van de structurele netwerken nader bekeken. Verschillen in graaf maten tussen gezonden en patiënten waren aanwezig en geassocieerd met cognitieve parameters. Wederom werden bij de patiënten die cognitief het meest aangedaan waren de voornaamste afwijkingen gevonden. De groep met jonge patiënten is onderzocht in hoofdstuk 5. De resultaten lieten zien dat de afwijkingen in de functionele netwerken van de patiënten vooral gekarakteriseerd werden door een vermindering in connectiviteit tussen subnetwerken (de modules). De verminderde tussen-module connectiviteit was het meest uitgesproken bij de patiënten met lage cognitieve scores. Bovendien liet vooral de frontaalkwab een andere modulaire organisatie zien bij de patiënten met frontaalkwab epilepsie. Ook viel er een module te identificeren die bij gezonde personen een grote uitgebreidheid had en vele kwabben bestreek, die echter in patiënten veel minder uitgebreid bleek, vooral in die patiënten die cognitief aangedaan waren. In hoofdstuk 6 is onderzocht of de functionele afwijkingen die bij de kinderen aanwezig waren ook te zien waren in de structurele netwerken. Er werd bij de patiënten een afname in tussen-module connectiviteit en een toename in modulariteit, pad lengte en clustering waargenomen. Opvallend was dat de opbouw van de structurele netwerken nauwelijks of geen verandering liet zien, maar dat er wel sprake was van een algemene verhoging van de structurele (witte stof) connectiviteit.

De resultaten van het onderzoek gepresenteerd in deze thesis laten zien dat er een belangrijke associatie is tussen afwijkingen in macroscopische hersennetwerken en cognitieve stoornissen bij patienten met lokalisatie gebonden epilepsie. Wanneer de veranderingen in hersennetwerken, zoals gemeten met MRI en graaf maten, eerder zouden optreden dan veranderingen in neuropsychologische uitkomsten (die de cognitieve achteruitgang meten), zou vroege detectie van patienten die cognitieve stoornissen gaan krijgen mogelijk zijn. Een vroege detectie van een patient met een verhoogd risico op cognitieve stoornissen kan waardevol zijn in de behandeling van 
epilepsie, omdat een vroege en adequate therapeutische interventie zou kunnen leiden tot een betere cognitieve uitkomst op de lange termijn. Dit is vooral belangrijk bij kinderen met epilepsie, aangezien de kinderen en ouders in het ongewisse verkeren of een behandeling zal aanslaan en wat het opleidingspotentieel zal worden. 


\section{Dankwoord}




\section{DANKWOORD}

Hier zit ik dan in ons nieuwe huis nabij Geneve. Met een been in het nieuwe avontuur, terwijl mijn promotie nog niet eens helemaal afgerond is. Maar ik zou hier natuurlijk niet zitten zonder de steun van een hoop mensen. Een aantal daarvan wil ik in het bijzonder bedanken.

Beste Walter, een van de eerste dingen die je me vertelde was dat je deur altijd openstond en dat ik minstens drie keer per week binnen moest lopen met vragen. Het duurde even voordat ik doorhad dat je dat zowel letterlijk als figuurlijk echt meende. Je had op een of andere manier altijd tijd voor me (ondanks je vele andere verplichtingen). Heel veel dank voor je inzet en alles wat ik van je heb mogen leren. Je werklust en passie voor de wetenschap zijn een inspiratie voor me.

Beste Bert, als promotor was je nauw betrokken bij mijn onderzoek. Als 'techneut' moest ik even wennen aan het gedragwetenschappelijk en klinisch onderzoek, maar jouw leiding aan onze onderzoekslijn en je klinische interpretatie van de resultaten hebben me altijd het vertrouwen gegeven dat ik op de goede weg zat.

Beste Paul en Jaap, jullie bijdragen aan mijn promotie waren onmisbaar. Paul, bedankt voor je blijvende interesse in mijn onderzoek. Ik was altijd erg onder de indruk van je kennis op het gebied van epilepsie en de neuroradiologie. Jaap, hartelijk dank voor je betrokkenheid bij mijn promotie (zelfs toen je in New York zat). Je zit altijd vol goede ideeën en ik kon altijd bij je terecht voor vragen.

Beste Hilde en Mariëlle (mijn "klinische partners"), het was een plezier om met jullie samen te werken. Hilde, ik vind het erg fijn dat je nu ook als mijn paranimf wil optreden. Ik weet zeker dat we de nog liggende stukken van onze IMAGINE studie tot een succes zullen maken. Mariëlle, bedankt dat je me wegwijs hebt gemaakt in de CODICE studie en het schrijven van een klinisch getint artikel, daar heb ik enorm van kunnen profiteren!

Beste René, lange tijd was je naast mij de enige AIO op het neuro-imaging vakgebied en ik vond het erg fijn om met je van gedachte te kunnen wisselen over het brein en frustraties op het gebied van NIFTI headers en beeldtransformaties te kunnen delen. Leuk dat je nu ook als paranimf naast me wil staan. Beste Marc en Jos, zonder jullie hulp op soft- en hardware gebied was ik nu waarschijnlijk nog steeds analyses aan het uitvoeren op mijn oude Windows PC. Dankzij jullie heb ik veel geleerd over scripting en parallel processing, iets dat me zeker van pas zal komen in de toekomst. Alle oude en nieuwe collega's van radiologie en Kempenheaghe: Eline, Gerrit, Doenja en Monique, Robert, Bas, Robert-Jan, Frank, Karolien, Marlies, Martijn, Kim, Vera, Eline, Frank, Bart, Martine, Jorn, Sander, Hanke, Cecile, Ewelina, Christine, Petra, Lucas, Raf, 
bedankt voor de leuke tijd. Beste Esther, Petra en Remco, zonder jullie inzet bij de inclusie en het scannen was de IMAGINE studie niet mogelijk geweest. Bedankt! Beste prof. Wildberger, bedankt voor de mogelijkheden die u me heeft geboden en dat ik de afgelopen jaren in alle vrijheid in het azM bij de afdeling radiologie heb mogen werken.

Laura en Merlijn, jullie zijn bijna ons spiegelbeeld! Klimmen, promoveren en een postdoc in het buitenland (en dan ook nog in hetzelfde gebied!). Ik hoop dat we de komende jaren nog veel leuke dingen blijven doen en werk en blessure leed kunnen blijven delen. Boris, partner in crime, samen blokken voor de OR master en daarna eerst even het bedrijfsleven in en dan toch maar voor de wetenschap kiezen. Bedankt voor alle DE-tjes (volgens mij heb jij er uiteindelijk meer betaald...).

Mijn lieve familie en ook die van llona wil ik heel erg bedanken voor alle steun door de jaren heen. Lieve pap en mam, dankzij jullie ben ik wie en waar ik vandaag ben. Jullie onvoorwaardelijke steun, liefde en aanmoediging zijn onmisbaar voor me. Anniek, Bram en Thijs, Paul en Dyon, het is belangrijk om een familie te hebben waar je op terug kunt vallen en dankzij jullie weet ik dat ik altijd op een gezellige tijd thuis kan rekenen. Marius en Marijke, Mark en Karina, jullie ook bedankt voor de gezellige momenten en interesse.

Lieve llona, voor jou is de laatste plek gereserveerd, de ere plek. Alles wat ik in woorden zou kunnen vatten, zou je tekort doen. Ik hoop dat je weet hoeveel je voor me betekend. Bedankt... voor alles! Ik hou van je. 
List of publications 


\section{PUBLICATIONS}

1) M.J. Vaessen, P.A.M Hofman, H.N. Tijssen, A.P. Aldenkamp, J.F.A. Jansen, W.H. Backes. The effect and reproducibility of different clinical DTI gradient sets on small world brain connectivity measures. Neuroimage. 2010: 51(3):1106-16

2) M.J. Vaessen, M.C.G. Vlooswijk, J.F.A. Jansen, M.C. De Krom, H.J. Majoie, P.A.M. Hofman, A.P. Aldenkamp and W.H. Backes. Loss of network efficiency associated with cognitive decline in chronic epilepsy. Neurology. 2011, 77(10):938-44

3) H.M.H. Braakman, M.J. Vaessen, P.A.M. Hofman, M.H.J.A. Debeij-van Hall, W.H. Backes, J.S. Vles, A.P. Aldenkamp. Cognitive and behavioral complications of frontal lobe epilepsy in children: A review of the literature. Epilepsia. 2011: 52(5):849-56

4) M.J. Vaessen, J.F.A. Jansen, M.C.G. Vlooswijk, P.A.M. Hofman, H.J. Majoie, A.P. Aldenkamp, W.H. Backes. White matter network abnormalities are associated with cognitive decline in chronic epilepsy. Cerebral Cortex 2011; doi: 10.1093/cercor/bhr298, Epub ahead of print

5) S.J. van der Kruijs, N.M. Bodde, M.J. Vaessen, R.H. Lazeron, K. Vonck, P. Boon, P.A.M. Hofman, W.H. Backes, A.P. Aldenkamp, J.F.A. Jansen. Functional connectivity of dissociation in patients with psychogenic non-epileptic seizures. Journal of Neurology Neurosurgery and Psychiatry. 2011, 83(3):239-47

6) H.M.H. Braakman, D.M. IJff, M.J. Vaessen, M.H.J.A. Debeij-van Hall, P.A.M. Hofman, W.H. Backes, J.S.H. Vles, A.P. Aldenkamp. The cognitive and behavioural profile of children with frontal lobe epilepsy. Submitted

7) M.J. Vaessen, H.M.H. Braakman, J.S. Heerink, J.F.A. Jansen, M.H.J.A. Debeij-van Hall, P.A.M. Hofman, A.P. Aldenkamp, W.H. Backes. Abnormal modular organization of functional networks in cognitively impaired children with frontal lobe epilepsy. Cerebral Cortex, under revision

8) R.M.H. Besseling, J.F.A. Jansen, G.M. Overvliet, M.J. Vaessen, H.M.H. Braakman, P.A.M. Hofman, A.P. Aldenkamp, W.H. Backes. Tract specific reproducibility of tractography based morphology and diffusion metrics. PLOS ONE, accepted

9) M.J. Vaessen, H.M.H. Braakman, P.A.M. Hofman, A. de Louw, A.P. Aldenkamp, J.F.A. Jansen, W.H. Backes. Connectional abnormalities of functional and structural networks in childhood frontal lobe epilepsy. In preparation 
182 
Curriculum Vitae 


\section{CURRICULUM VITAE}

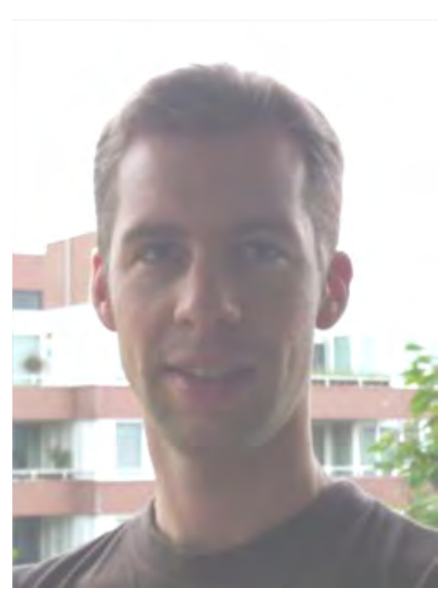

Maarten Vaessen was born on July $4^{\text {th }} 1981$ in Hoensbroek, the Netherlands. He completed secondary school at the Sinter Meerten College in Heerlen in 1999. After short endeavors at Computer Science in Heerlen and Delft, he started studying Knowledge Engineering at the Maastricht University in September 2002. There, he finished his Bachelor of science in July 2005 with a thesis on ECG waveform detection using the analog wavelet transform. In September 2005, he started studying for a Master of Science degree in Operations Research at the Maastricht University. At the department of Instrument Development Engineering \& Evaluation, Maarten fulfilled an internship on the topic of ECG delineation using wavelet analysis and hidden Markov models under supervision of Dr. R. Westra. He obtained his master's degree cum laude in October 2006. During his studies, Maarten's interest in biomedical signal analysis was kindled. Subsequently, he worked for one year as a software engineer at PIE Medical Imaging in Maastricht. In January 2008 he decided to start a PhD project at the Department of Radiology, Maastricht University Medical Centre and the Epilepsy Institute Kempenhaeghe in Heeze. He investigated brain connectivity in epilepsy patients using advanced Magnetic Resonance Imaging techniques under supervision of Prof. Dr. A. Aldenkamp, Dr. Ir. W. Backes and Dr. P. Hofman. During his PhD, Maarten's work was rewarded with student stipends and presentations at international conferences in addition to several poster prizes.

In April 2012, Maarten will start as a post-doctoral fellow at the Laboratory for Neurology \& Imaging of Cognition at the University of Geneva, Switzerland. 